\title{
Laser-Material Interaction of Powerful Ultrashort Laser Pulses
}

\author{
A. M. Komashko \\ Doctor of Philosophy in Applied Science
}

January 6, 2003

U.S. Department of Energy

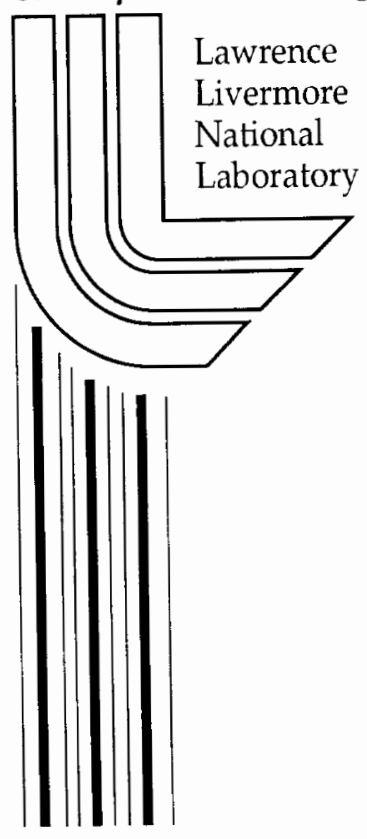




\section{DISCLAIMER}

This document was prepared as an account of work sponsored by an agency of the United States Government. Neither the United States Government nor the University of California nor any of their employees, makes any warranty, express or implied, or assumes any legal liability or responsibility for the accuracy, completeness, or usefulness of any information, apparatus, product, or process disclosed, or represents that its use would not infringe privately owned rights. Reference herein to any specific commercial product, process, or service by trade name, trademark, manufacturer, or otherwise, does not necessarily constitute or imply its endorsement, recommendation, or favoring by the United States Government or the University of California. The views and opinions of authors expressed herein do not necessarily state or reflect those of the United States Government or the University of California, and shall not be used for advertising or product endorsement purposes.

This work was performed under the auspices of the U.S. Department of Energy by the University of California, Lawrence Livermore National Laboratory under Contract No. W-7405-Eng-48.

This report has been reproduced directly from the best available copy.

Available electronically at http://www.doe.gov/bridge

Available for a processing fee to U.S. Department of Energy and its contractors in paper from

U.S. Department of Energy Office of Scientific and Technical Information P.O. Box 62

Oak Ridge, TN 37831-0062

Telephone: (865) 576-8401

Facsimile: (865) 576-5728

E-mail: reports@adonis.osti.gov

Available for the sale to the public from

U.S. Department of Commerce

National Technical Information Service 5285 Port Royal Road Springfield, VA 22161

Telephone: (800) 553-6847

Facsimile: (703) 605-6900

E-mail: orders@ntis.fedworld.gov

Online ordering: http://www.ntis.gov/ordering.htm

OR

Lawrence Livermore National Laboratory

Technical Information Department's Digital Library

http://www.llnl.gov/tid/Library.html 
Laser-material interaction of powerful ultrashort laser pulses

by

Aleksey Mikhaylovich Komashko

B.S. Novosibirsk State University, Novosibirsk, Russia 1995

M.S. Novosibirsk State University, Novosibirsk, Russia 1997

\section{DISSERTATION}

Submitted in partial satisfaction of the requirement for the degree of DOCTOR OF PHILOSOPHY

in

Applied Science

in the

OFFICE OF GRADUATE STUDIES

of the

UNIVERSITY OF CALIFORNIA

DAVIS

Approved:

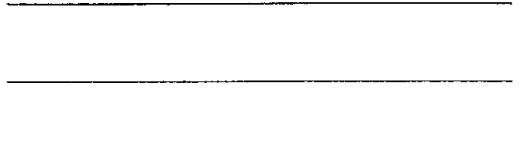

Committee in Charge

2003 
Aleksey Mikhaylovich Komashko

March 2003

Applied Science

\title{
Laser-material interaction of powerful ultrashort laser pulses
}

\begin{abstract}
Laser-material interaction of powerful (up to a terawatt) ultrashort (several picoseconds or shorter) laser pulses and laser-induced effects were investigated theoretically in this dissertation. Since the ultrashort laser pulse (USLP) duration time is much smaller than the characteristic time of the hydrodynamic expansion and thermal diffusion, the interaction occurs at a solid-like material density with most of the light energy absorbed in a thin surface layer. Powerful USLP creates hot, high-pressure plasma, which is quickly ejected without significant energy diffusion into the bulk of the material. Thus collateral damage is reduced. These and other features make USLPs attractive for a variety of applications. The purpose of this dissertation was development of the physical models and numerical tools for improvement of our understanding of the process and as an aid in optimization of the USLP applications.

The study is concentrated on two types of materials - simple metals (materials like aluminum or copper) and wide-bandgap dielectrics (fused silica, water). First, key physical phenomena of the ultrashort light interaction with metals and the models needed
\end{abstract}


to describe it are presented. Then, employing one-dimensional plasma hydrodynamics code enhanced with models for laser energy deposition and material properties at low and moderate temperatures, light absorption was self-consistently simulated as a function of laser wavelength, pulse energy and length, angle of incidence and polarization.

Next, material response on time scales much longer than the pulse duration was studied using the hydrocode and analytical models. These studies include examination of evolution of the pressure pulses, effects of the shock waves, material ablation and removal and three-dimensional dynamics of the ablation plume.

Investigation of the interaction with wide-bandgap dielectrics was stimulated by the experimental studies of the USLP surface ablation of water (water is a model of biological tissue) and laser-induced pressure waves. Simulations on the basis of the nonlinear ionization equation were used to examine effects of the laser created surface plasma on light absorption, reflection and transmission. Laser pulse energy conversion efficiency into pressure waves was studied experimentally and theoretically. 


\section{Acknowledgements}

I was often privileged to meet and work with people, who were not just the best in the field, but who were also unselfishly interested in guiding and helping me in my scientific and personal quests. Here I would like to acknowledge those who played an important role during my graduate school years.

I often shared offices with Sasha Rubenchik. Fortunately, this is not the only thing he shared. Sasha helped me in innumerous ways. He willingly or inadvertently taught me a lot about science and life too. True appreciation for new knowledge and difficulty of discovering it in an imperfect world is something that I acquired under his influence. I hope he enjoyed my company as much as I did his.

My laboratory adviser Mike Feit always led by example. Precision of his thought and its expression, ability to detach thinking from emotions, his practical, yet not loosing the bigger picture opinions were very inspiring. Mike's words of encouragement meant a lot for me. Mike and Sasha shielded me from large organization's bureaucratic machine as much as possible. Certainly, years later, I will remember this time as a carefree "scientific childhood". My academic advisor Rick Freeman also helped me to stay focused on my work and not to worry about distractions. His optimism supported my belief in brighter and better future. 
Special thanks to Jon Larsen who patiently implemented my countless suggestions in his code. Despite a constant stream of requests and complains, he was always willing to take on new things. Without Jon's efforts this dissertation would have been something else.

Significant part of my thesis is a result of work with Beop-Min Kim. Hopefully the benefit was mutual and I would like to thank him for his patience.

Financial support and research opportunities at the Lawrence Livermore National Laboratory were very appreciated.

Finally, I would like to acknowledge influence of my parents. The values they instilled in me, their support and encouragement took me where I am now. Thank you. 


\section{Table of contents}

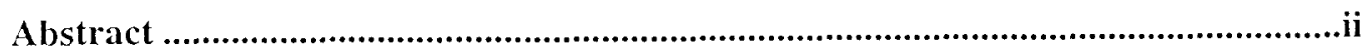

Acknowledgements....................................................................................................

Table of contents .....................................................................................................

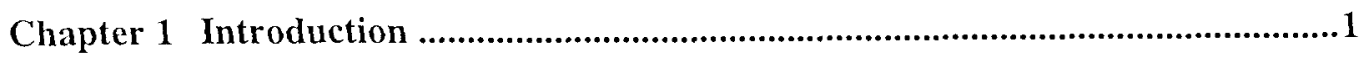

1.1 Powerful ultrashort laser pulse systems ….............................................. 1

1.2 Interaction of ultrashort powerful light pulses with materials .......................4

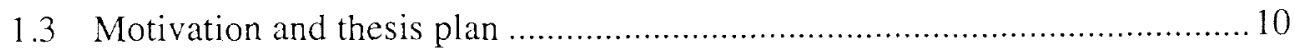

Chapter 2 Ultrashort laser pulse interaction with simple metals: theory and models employed 16

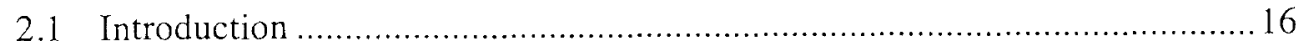

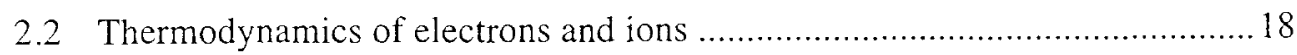

2.3 Two temperature model of laser energy absorption ...................................22

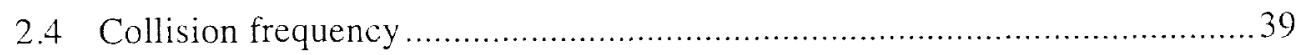

2.5 Optical properties of metals and light absorption........................................ 45

2.6 HYADES - hydrodynamics code for self-consistent modeling of USLP laser-

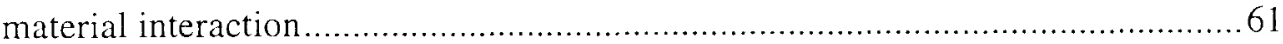

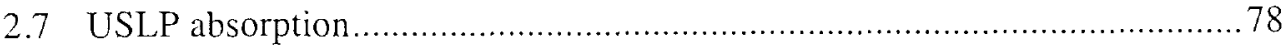

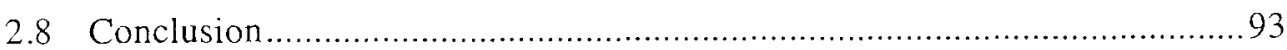

Chapter 3 Long-term effects of ultrashort laser-material interaction .....................96

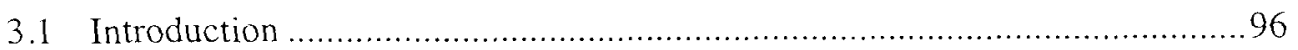

3.2 Effects of ultrashort and short pulse interaction ....................................... 98

3.3 Energy transport and deposition by shock waves................................... 102

3.4 Evolution of USLP generated shock waves ......................................... 116

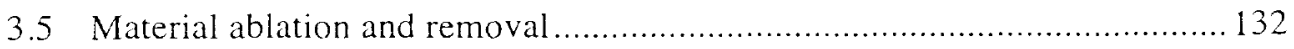


3.6 Three-dimensional evolution of the ablation plume

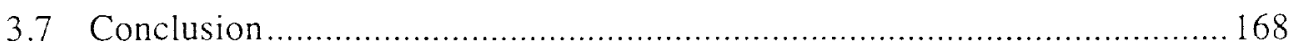

Chapter 4 Ultrashort laser pulse interaction with wide-bandgap dielectrics........ 171

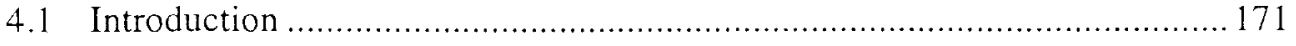

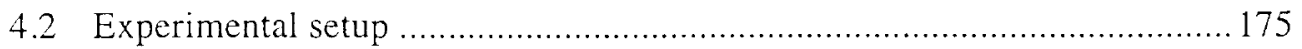

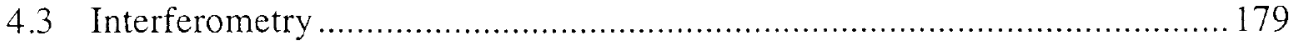

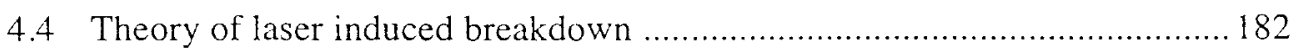

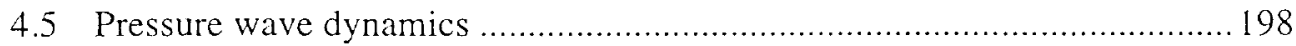

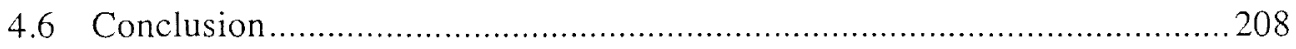

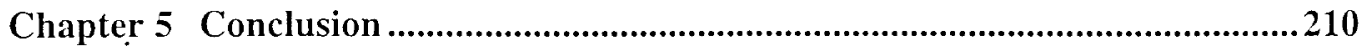

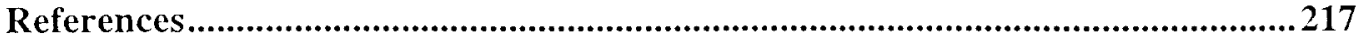




\section{Chapter 1 Introduction}

\subsection{Powerful ultrashort laser pulse systems}

Energetic, precisely controlled beams of light, a fixture of science fiction for a long time [Wells02,Tolstoy01], became a reality with the first demonstration of the laser in 1960 [Maiman60]. In the past four decades studies of the interaction of optical radiation with matter became one of the most active and visible branches of physics and the laser established itself as an indispensable and versatile tool in many applications. A very large and diverse group of applications such as material processing [Miller98,Baurle00], inertial confinement fusion [Basov63,Lindl95,Yamanaka91], missile defense [Roden00,Dane02] can be classified as applications where light is used as an energy source. Several laser characteristics make it attractive for this role. High beam directionality and light monochromaticity allow very fine spatial control over energy delivery - a good quality beam can be focused in a spot size comparable to a light wavelength (approximately $0.5 \mu \mathrm{m}$ in the visible range). Adjusting laser pulse length and shape, one can simultaneously exercise high control in temporal energy delivery. Laser power is another very flexible parameter - it can be as small as several $\mathrm{mW}$ in a personal CD recorder [Starret00] or it can be as large as 500 TW in the National Ignition Facility (NIF) [Murray98]. 
High power and, therefore, high intensity and electric field is one the most desirable laser parameters. Unfortunately, it usually comes with a high cost. For example NIF is a one of a kind, billion dollar facility larger than a football field. Regular pulsed laser systems can typically generate several GW of peak power in a pulse few nanoseconds long. One could try to achieve higher peak power by extracting laser amplifier energy in a shorter pulse, however it becomes progressively more difficult when the pulse length decreases to the picosecond or femtosecond regime. In order to have efficient extraction of stored energy, pulse fluence (light energy density per unit of transverse area) has to be on the order of the so called saturation fluence that, for a short pulse, would correspond to significant laser intensity. Since the refractive index of the medium usually has a term proportional to the light intensity $I: n=n_{0}+n_{2} I$, amplification of high power laser pulses creates nonlinear phase retardation that results in wave-front distortion and eventual catastrophic collapse in material damaging light filaments [Shen75,Marburger75,Shen84].

This limitation was circumvented with the development of Chirped Pulse Amplification (CPA) first demonstrated by Strickland and Mourou in 1985 [Strickland85]. The technique is based on an idea borrowed from radar technology. If a short pulse is transmitted though a dispersive medium, different spectral components travel at different speed, thus stretching the pulse. Simultaneously the pulse becomes chirped - light frequency (color) changes along the pulse. After stretching, the laser pulse 
has low intensity and long length that permit safe and efficient amplification to a high energy. Sending the pulse through a medium with the opposite sign of dispersion recompresses it to its initial, short length. To make this work in optical spectrum, Strickland and Mourou used a single mode optical fiber to generate positive dispersive delay and a double diffraction grating compressor, proposed by Treacy [Treacy69] (see Figure 1.1), to create negative dispersion. Later, it was shown [Martinez87, Maine88] that a pair of gratings could also be used as a stretcher. This allows a very good match of positive and negative dispersions, thus the stretching-compression ratio can reach $10^{4}$ or higher without creation of temporal distortion. As a result, it is possible to generate femtosecond pulses with TW power from a tabletop system [Perry94,Backus98, Mourou98]. Larger lasers can be operated in a petawatt or higher regime [Perry99, Pennington00].

The short pulse generator (oscillator) and broadband lasing material, in particular, determine the light wavelength and pulse length of a CPA system. A modern CPA laser is typically based on a solid-state lasing material. There is a variety of such materials suitable for short pulse generation, however one of the most widely used is Ti:sapphire [Spence91], with tuning band from $660 \mathrm{~nm}$ to $1100 \mathrm{~nm}$. Other available materials are Nd:glass (1.043um-1.063um) [Strickland85,Maine88] or Cr:LiSAF (750nm-950nm) [Ditmire96]. Oscillators based on Ti:sapphire are typically operated at $800 \mathrm{~nm}$ wavelength 
and can generate pulses as short as 10fs. Since it is often advantageous to have high repetition rate systems, Ti:sapphire laser can be operated at a kilohertz rate with

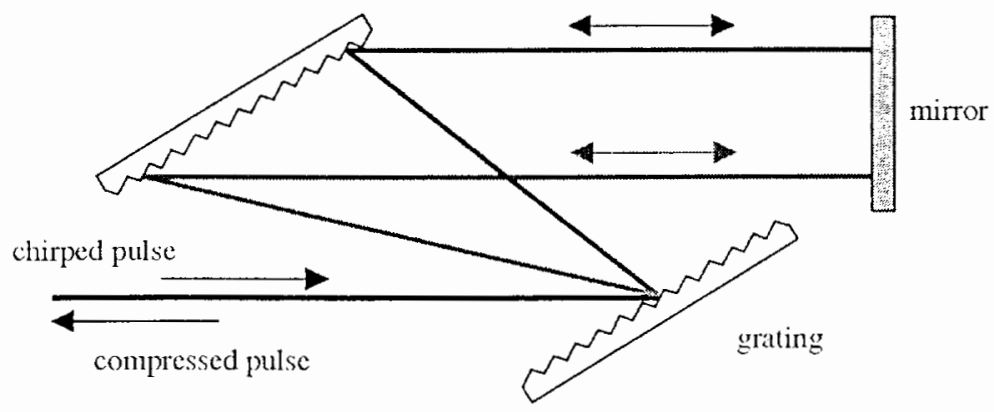

Figure 1.1: Double grating compressor of Treacy [Treacy69]. Since reflection angle of a nonzero diffraction order is a function of light wavelength, arrangement of two gratings shown on the picture creates different paths for different spectral harmonics of the laser pulse. Red, longer wavelength component has to travel larger distance than blue, shorter wavelength component, effectively creating system with negative dispersion.

an average power of several watts [Backus97,Nabekawa98,Bagnoud00] (pulse energy is several millijoules). Such lasers can generate pulses as short as tens of femtoseconds (optical cycle length at $800 \mathrm{~nm}$ wavelength is $2.7 \mathrm{fs}$ ), which is extremely short when compared to the previous systems that generated nanosecond pulses. Thus it is quite common to refer to them as to ultrashort or ultrafast lasers.

\subsection{Interaction of ultrashort powerful light pulses with materials}

The combination of high power and short pulse length puts the interaction of ultrashort laser light with materials into a unique physical regime. The main distinction comes from the changes in the way laser energy is absorbed. Details of energy deposition 
are material specific; however, since deposition is mostly driven by the properties of electrons, all materials can be roughly divided in two groups: metals and dielectrics.

A fundamental feature of metals is the presence of significant number of electrons that behave like free particles. These electrons are predominantly responsible for thermal, electrical and often optical properties of a metal. Their collective response to a laser light will be similar to that of a cold plasma [Kitte196], where a transverse electromagnetic wave can propagate only if the light frequency $\omega_{0}=2 \pi c / \lambda$ is larger the than plasma frequency [Spitzer62] $\omega_{i}=\sqrt{4 \pi n_{e} e^{2} / m_{e}}$, where $c$ is the speed of light, $\lambda$ is the light wavelength, $n_{e}$ is the electron density, $e$ is the electron charge, and $m_{e}$ is the electron mass (cgs units are adopted). This requirement is equivalent to the condition that the laser beam can propagate only if the electron density is below the so called critical density $n_{c r} \sim 10^{21} \mathrm{~cm}^{-3} / \lambda^{2}$, where $\lambda$ is in microns. For lasers in the ultraviolet, visible or near infrared ranges this formula gives $n_{c r} \sim 1 \div 10 \cdot 10^{21} \mathrm{~cm}^{-3}$. Since the typical "free" electron density in a metal is on the order of $10^{23} \mathrm{~cm}^{-3}$ [Kittel96], the dielectric constant $\varepsilon \sim 1-n_{\mathrm{c}} / n_{\mathrm{cr}}$ is negative. Therefore, a significant portion of the incident energy will be reflected and the rest is absorbed in a thin surface layer (skin depth) with thickness on the order of $10 \mathrm{~nm}$ [Palik98].

Laser beams produced by a typical short, nanosecond system can have energy on the order of a joule [Spectra02]. Focusing such a pulse in a spot hundreds of microns in 
size results in a fluence of $10 \mathrm{~kJ} / \mathrm{cm}^{2}$ or higher. For this large fluence, material evaporation begins well before the pulse peak. Hot vapor expands with velocity on the order of the thermal velocity $v_{t}=\sqrt{3 k_{B} T / A m_{p}}$, where $T$ is the vapor temperature, $k_{b}$ is the Boltzmann constant, $A$ is the atomic number and $m_{p}$ is the proton mass. For a material like aluminum, temperature of few thousand degrees translates into velocities of several $\mathrm{km} / \mathrm{s}$. After one nanosecond, products of evaporation will transform into a plume several microns thick. Critical density simultaneously moves away from the solid material and, as a result, most of the laser energy is absorbed in the expanding cloud (plasma shielding effect). The plume acquires significant energy and momentum and its recoil pressure launches a strong shock wave into the material. Thermal diffusion is another mechanism of energy transport in the bulk. Thermal wave penetration distance is on the order of the diffusion length $\sqrt{\chi \tau / C}$ [Rykalin78,Prokhorov90,Carslaw86], where $\chi$ is the thermal conductivity, $C$ is the heat capacity, and $\tau$ is the pulse length. For a typical metal the thermal diffusivity $\chi / C$ is $0.1 \div 1 \mathrm{~cm}^{2} / \mathrm{s}$ [Prokhorov90,Gray63]. Thus a nanosecond pulse will heat a surface layer hundreds of nanometers thick - much larger than skin depth of a metal.

This picture changes considerably for ultrashort laser pulses (USLP). Decrease of three or four orders of magnitude in the pulse length produces a drastic, qualitative transformation of the laser material interaction - in which light energy absorption zone 
simply does not have enough time for a considerable expansion so most of the interaction occurs at solid-like material densities. In fact, expansion, even at a very high velocity of $10^{7} \mathrm{~cm} / \mathrm{s}$ after exposure by a $100 \mathrm{fs}$ pulse, creates a plume only $10 \mathrm{~nm}$ thick - comparable to the skin depth of a metal at normal conditions. Besides straightforward effects of pulse shortness there is a new physical mechanism reflecting the uniqueness of USLP. Due to the high mobility of electrons, they are the predominant energy absorbers. However material movement begins only if the ions are heated too. Simple analysis of two-particle collisions shows that the energy exchange time is proportional to the mass ratio $m_{e} / m_{i}=m_{e} / A m_{p}[$ Landau76, Ginzburg55]. Thus, the electron-ion mass disparity results in a relatively slow energy transfer from electrons to ions - the characteristic temperature equilibration time in a metal is on the order of tens of picoseconds [Kaganov57,Anisimov74]. As a result, material expansion begins only after the USLP is over. Thermal penetration during equilibration is tens of nanometers, again a number comparable to the skin depth of a metal. Since energy deposition is confined in a very thin layer, even millijoule energies of typical ultrafast laser systems are enough to heat the material up to tens of thousands of degrees or higher (a more convenient unit to measure temperature is the energy unit electronvolt, $\left.1 \mathrm{eV}=1.6 \cdot 10^{-12} \mathrm{erg} \rightarrow 11600^{\circ} \mathrm{K}\right)$. High temperature creates significant pressure that initiates fast material expansion. As a result, extremely hot ejecta containing most of the laser deposited energy quickly moves away 
from the bulk. Therefore energy deposition outside of the absorption layer is reduced. It is quite common to refer to this process as laser ablation. The term ablation is used in a variety of disciplines and usually describes material removal by some means [MWebster02]. In this case, it is a laser induced material removal via an energetic, fast expanding plume.

Laser interaction with dielectrics is noticeably different because of the absence of "free" electrons. The energy gap between the electron valence and conduction bands can be considerably larger than the photon energy (photon energy $\hbar \omega_{0}=\frac{1.24}{\lambda[\mu \mathrm{m}]}[\mathrm{eV}] \sim 1 \div 3 \mathrm{eV}$ for visible or near infrared light; the bandgap energy of, for instance, fused silica (glass) is $9 \mathrm{eV}$, sapphire $-8.7 \mathrm{eV}$ [Palik98,DeSalvo96]) so ionizing transitions from band to band via one photon absorption are prohibited. As a result, dielectrics under normal conditions are poor light absorbers and poor conductors. Nonetheless, it is possible to perform ablation with long pulses mostly due to the fact that there is always some small number of "free" electrons that are contributed by defects, impurities, thermal ionization, etc.. Once these electrons acquire enough energy from the laser, they begin to ionize the material through collisions with atoms or molecules. This creates more electrons that, in turn, participate in the process. Since every electron can go through multiple acts of ionization, the whole process is exponential, and it is called avalanche ionization. Depending on the beam energy, length, and focusing, significant electron density can be 
reached during the pulse. Typically many "generations" of avalanche multiplication are required to reach high electron density. At this point direct laser heating (like in metals) becomes important and noticeable material modification or ablation is observed. This is the so-called laser induced breakdown [Bloembergen74], which is typically described in terms of a damage or ablation threshold.

Since initiation of avalanche ionization relies on "seed" electrons generated by random effects (defects, etc.), long pulse ablation is a probabilistic process. The ablation threshold is a function of material quality so it is difficult to tell a priori at what fluence specific material sample can be ablated. In ultrashort ablation, this problem is avoided altogether by employing very high laser intensity of USLP. Initial, seed electrons can be created via simultaneous absorption of several photons. The process is called multiphoton ionization (MPI) and it is a strong function of laser intensity $I$ [Keldysh65]. The ionization rate is proportional to $I^{k}$, where $k$ is the number of photons needed to bridge the energy bandgap (for fused silica and $800 \mathrm{~nm}$ light $k=6$ ). Since seed electrons are created by MPI, which is a function of bandgap energy - an intrinsic property of the material, the interaction becomes deterministic. The strong intensity dependence of the ionization allows fine control over spatial details of interaction and produces sharp ablation thresholds.

Absorption of ultrashort pulses by wide-bandgap dielectrics and metals represent two extreme cases of the laser material interaction. There is a variety of other energy 
deposition mechanisms, for example electron transitions from valence to conduction band via one photon absorption are especially important in semiconductors (energy bandgap in $\mathrm{Si}$ is $1.11 \mathrm{eV}$, in $\mathrm{Ge}$ is $0.66 \mathrm{eV}$ [Kittel96]). Details of the absorption are material specific. However, there are features that will be present in ultrashort ablation of any material. These include light absorption at solid-like densities, production of high temperature plumes, small residual energy deposition, uniform and deterministic ablation.

\subsection{Motivation and thesis plan}

The modern ultrashort pulse laser is a compact, tabletop system capable of delivering femtosecond, powerful pulses at a high repetition rate. Interaction of USLPs with materials has many features that make ultrashort laser systems attractive for a variety of applications. The most obvious application is high precision material processing (cutting, drilling, microstructuring). Since most of the USLP deposited energy is carried away by the thin surface material layer turned into ejecta, it is possible to achieve micron or better accuracy of processing with low collateral damage [Chichkov96,Nolte97,Liu97,Perry99a]. Low thermal and mechanical energy deposition in the bulk of the material is especially important in biomedical applications [Neev96,Loese196,Loese198]. It is even possible to perform safe cutting of explosives [Perry99a]. Material ablation occurs through creation of high temperature plasma, which is relatively short lived. This plasma can be used as an intense, short pulse X-ray source 
[Kieffer93,Murnane94]. Spectroscopy of the ablation plume can be used for material analysis [Margetic00,Drogoff01] and USLP is especially attractive for that, since the amount of material removed per pulse is small, thus it is practically nondestructive. Lasers are also used for manufacturing of unique thin films [Miller98,Bauerle00] by deposition of atoms and molecules from the ablation plume onto a substrate. Obtaining film homogeneity in conventional pulsed laser deposition is difficult because of ejection of macroscopic particles, that are created by melting and splashing of the material from the ablation surface. USLP ablation creates a very small amount of melt, thus making it attractive for film deposition [Banks99,Okoshi00].

Understanding physics of interaction is important for advancing scientific knowledge in a new regime and to aid application to technology. However, a theoretical, quantitative description of ultrashort pulse laser-material interaction and its effects is lacking. The physics of the process is involved and complex. Light propagation and absorption, material expansion, thermal and radiation transport, ionization, and this is only a partial list, must be described self-consistently on greatly changing temporal and spatial scales. Analytical solution of this problem is out of reach in all, but the simplest cases. Numerical calculations are the only viable option. Another difficulty is that there is no simple and agreed upon theoretical prescription on how to solve this problem. A full theoretical description is too complex. The purpose of this dissertation is development of 
simple physical models for numerical simulation of USLP ablation in a significant range of laser parameters and their application.

The primary subject of the modeling is the interaction of pulses from a commonly used ultrashort laser system that typically produces pulse lengths in the picosecond and subpicosecond range and energies up to tens of millijoules with available power up to a terawatt. In this regime, interaction with both cold material and hot plasma is important, as opposed to petawatt pulses, which have enough energy in the prepulse to produce plasma, thus reducing material effects in the interaction of the main pulse. This regime of laser-plasma interaction is reviewed elsewhere [Gibbon96]. We are interested in lower energy interaction that will be referred to as laser-material interaction.

This study concentrates on two types of materials. The first group is near free electron (NFE) metals - materials like aluminum or copper, where details of the energy band structure are not very significant (as opposed to, for instance, iron) and "freely" moving electrons define interaction with USLP. The second group is wide bandgap dielectrics. Their response to ultrashort pulses is primarily driven by the presence of large energy gap (much larger than the photon energy) between conduction and valence bands, which makes nonlinear ionization central part of absorption process.

The next chapter is devoted to modeling of ultrashort pulse absorption by metals. The goal is to develop a flexible simulation method that captures the major physical phenomena of USLP absorption. As the laser heats up the metal, material transits from a 
cold solid to a hot, ideal plasma. The material state changes from being determined by the potential interaction to a state with properties controlled by the kinetic energy of the particles. Fortunately, since many of the simple (NFE) metal properties are dominated by the response of electrons that can be regarded as free particles, a uniform approach to the problem is possible. Our methodology is based on taking relevant, simple theoretical models for these two different states - solid and plasma, and interpolating between them in a physically consistent fashion.

The first section begins with a discussion of thermal properties, where emphasis is on the relative contribution of electrons and ions. Unequal temperature dynamics of electrons and ions is under consideration next. We show that in USLP interaction it can be considerably out of balance. Following is a review of collision frequency that plays a central role in calculations of thermal transport and optical properties. The importance of electron-electron collisions is emphasized. Discussion of models for calculation of the material dielectric function is next. The significance of interband transitions, even for a metal like aluminum, is demonstrated and a simple model to account for them is suggested. The physical models discussed are implemented on the basis of onedimensional plasma hydrodynamics code HYADES [Larsen94], which is described further in the text. This allows us to have a self-consistent description of USLP absorption that includes material expansion and ionization. Code benchmarking with available experimental data is presented and it shows that this approach works in a 
significant range of laser parameters. The final part of the chapter is a detailed investigation of USLP absorption. Effects of laser fluence, angle of incidence, and pulse length are discussed.

Energy deposition by USLPs is often an intermediary step. For most of the applications the interesting part is material behavior and properties long after the pulse is over. Due to disparity of temporal scales, this is a very challenging problem. Chapter 3 describes our efforts to treat it using a combination of the hydrocode and analytical methods. The first section opens the chapter with a comparison of ultrashort and short, nanosecond pulse interaction with a metal. The next section is concerned with the energy transported by shock waves. Shock wave material melting on compression and expansion is discussed. The calculations show that it might be a significant effect since it occurs at a shock pressure on the order of a few Mbars - pressure easily reached in USLP ablation. Modeling of the shock wave propagation is next. It is shown that evolution of major shock parameters is described by a power law dependence on time. Following is a discussion of ejecta plume structure. Our goal is to find criteria that, after a few nanoseconds of expansion, can give an estimate of the amount of material removed. Such criteria, based on the role of the liquid-vapor critical point, is demonstrated and applied in a range of calculations. The chapter is concluded by discussion of ejecta expansion modeling on a very long (microsecond) time scale. 
In Chapter 4, the discussion shifts to wide bandgap dielectrics with concentration on one specific problem - USLP surface ablation of water (a model of biological tissue) and shock wave generation. Characterization of the pressure waves is important for biological applications, since they are one of the ways in which tissue can be damaged. First, experimental, interferometric study of pressure waves is described. In order to explain results of the experiment, a model of material ionization and laser wave absorption and propagation is presented. The simulations show that the plasma created in the USLP surface ablation of dielectrics, and water in particular, reflects most of the pulse energy. However the leading edge of the laser pulse is transmitted into the bulk and the transmitted energy increases for longer pulses. Next ablation generated pressure wave strength and energy is estimated. We show, experimentally and theoretically, that in a near threshold USLP ablation the fraction of laser energy converted into mechanical form is small and the generated pressure waves are in the so called weak shock regime.

Chapter 5 concludes the dissertation, summarizes the results and describes possible future directions. 


\section{Chapter 2 Ultrashort laser pulse interaction with simple metals:}

\section{theory and models employed}

\subsection{Introduction}

Electrons, charged particles with a very small mass, dominate the response of a medium to an electromagnetic wave if they are free to move around and present in large numbers. This is the case for metals and ideal plasma. In the latter situation electrons are free by definition: ideal plasma is a gas of charged particles with the kinetic energy much larger than the potential energy of the interparticle interaction. This happens when matter is either at high temperature or low density (or both). Atoms and molecules in metals are much more closely packed and the potential interaction is significant - it causes matter to adopt a solid form. Nevertheless, the presence of free electrons is possible because of their wave properties. The low temperature, high density regime is described by quantum mechanical equations. The solution shows that, for a periodic arrangement of ions, the electron quantum states are grouped in material specific energy bands. Within each band the electron's energy can change continuously, as if there was no potential interaction. However, the electron is a fermion and can not occupy the same quantum state simultaneously with another electron. The only way to accommodate all electrons is to place them on different energy levels. After filling available phase space, a metal will 
have partially occupied bands, where electrons can change their energy continuously. Calculations and experiments show that among metals there is a large group of materials that can be classified as nearly free electron (NFE) metals (for example aluminum and copper, but not iron, that has a complicated band structure). Electrons in such metals can be viewed as free particles in a gas - their kinetic energy is described by a quadratic dispersion relationship $E \sim p^{2}$, where $p$ is particle momentum. This feature simplifies the description of many physical properties and allows a uniform treatment of NFE metals and plasma. In this chapter we present our efforts to model ultrashort laser pulse interaction with such metals for a wide range of laser parameters.

Laser ablation creates an expanding plume, which even on the nanosecond scale has a typical thickness much smaller than the transverse dimensions determined by the laser spot size. Therefore the problem can be viewed as one-dimensional. Our simulations are based on the statistical treatment of the material. Local thermal equilibrium is assumed; however, electrons and ions/phonons are treated as two energy-exchanging liquids that may have different temperatures. Modeling framework is provided by a onedimensional Lagrangian hydrodynamics code that allows description of material expansion and shock propagation. Temperature and density dependent ionization, thermal and radiation transport are part of the calculations. Laser energy deposition treatment includes wave effects and can be applied to either TE (S) or TM (P) polarized laser beams at an arbitrary angle of incidence. A major advantage of this approach is the ability 
to self-consistently model the response of the material to an ultrashort laser pulse (all of the significant physics is included and equations are solved simultaneously) on a variety of time scales (we track only macroscopic parameters, thus calculations are relatively inexpensive).

The chapter begins with a review and simple modeling of relevant physics with the purpose of illustration of key phenomena in the USLP interaction with NFE metals. It also serves as a demonstration of the models developed and implemented in the hydrocode, which is described next. Code benchmarking on the available experimental data of absorption of ultrashort laser pulses is also presented. Results and discussions of modeling of USLP absorption with the hydrocode for a variety of laser parameters conclude the chapter.

\subsection{Thermodynamics of electrons and ions}

Energy deposition in a metal by a typical USLP raises material temperature in a thin, surface layer. The range of achievable peak temperatures and penetration depths depends on many parameters. Heat capacity and thermal conductivity are among the most significant factors. Let's review available models and data.

The heat capacity of plasma is quite simple to understand. Since it is by definition a gas with weak interparticle interaction, the plasma internal energy $U$ is the sum of kinetic energies of all particles. Energy stored per particle is $\frac{3}{2} k_{B} T$ : each particle has 
three degrees of freedom, with $0.5 k_{B} T$ energy per degree according to the equipartition law [Landau69]. Therefore, the heat capacity of plasma $C_{V}=\partial U /\left.\partial T\right|_{V}$ is

$$
C_{V}=C_{V e}+C_{V I}=\frac{3}{2} k_{B}\left(n_{e}+n_{l}\right)=\frac{3}{2} k_{B} n_{l}(1+Z)
$$

where $n_{l}$ is density of ions and $Z$ is average ionization. For a hydrogen plasma $(Z=1)$ at the density $n_{l}=10^{21} \mathrm{~cm}^{-3}$, this gives $C_{V} \approx 0.04 \mathrm{~J} / \mathrm{K} \mathrm{cm}^{3}$. Plasma produced from a metal at solid density will have heat capacity $C_{V} \approx(1+Z) 1.5 \mathrm{~J} / \mathrm{K} \mathrm{cm}^{3}$ (typical atomic lattice size of a metal is $3 \AA$ [Gray63], this translates into ion density $n_{I} \sim 7 \cdot 10^{22} \mathrm{~cm}^{-3}$ ). At high temperatures, a significant number of electrons can be stripped from an atom of a metal, thus electrons dominate heat capacity of plasma in this regime.

For solid density materials at low temperature these simple arguments are no longer valid for description of electrons, since Boltzmann (Maxwellian) statistics can not be applied in this range of parameters and Fermi statistics must be used. Electrons are particles with fractional spin and can not simultaneously occupy the same quantum state with another electron. Therefore even for very small temperature they do not condense in low energy levels and can have significant average energy. Temperature in this case has a different meaning. In classical statistics, temperature is an average energy possessed by a particle. In the quantum case, it describes the energy of random motion and also serves as a measure of how many particles can participate in thermal processes (see Figure 2.1). According to the Fermi distribution formula 


$$
f(\varepsilon)=\frac{1}{e^{\left(\varepsilon-\mu(T) / k_{B} T\right.}+1}
$$

this description is necessary for $k_{B} T<<\mu(0)=\varepsilon_{F}$. In the reverse situation $k_{B} T>>\mu(T)$, Boltzmann statistics serves as a good approximation.

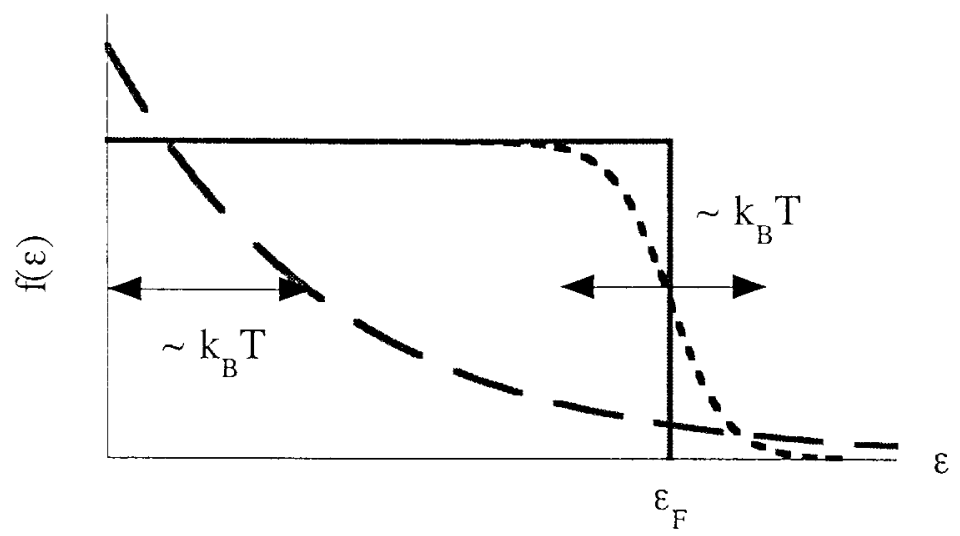

Figure 2.1: Schematic plot of Fermi distribution function $\left(\exp \left((\varepsilon-\mu) / k_{B} T\right)+1\right)^{-1}$ (solid red line - zero temperature, dashed blue line - nonzero temperature) and Boltzmann distribution $A \exp \left(-\varepsilon / k_{B} T\right)$ (dashed green line). In case of Fermi distribution particles fill up all of the states with energies up to approximately $\varepsilon_{F}-T$.

The Fermi energy $\varepsilon_{F}$ of a metal can be simply estimated if electrons are modeled as a degenerate gas, which is a good approximation in the NFE case [Kitte196,Landau69]. At zero temperature electron distribution has a trivial form: 1 for $\varepsilon<\varepsilon_{F}$ and 0 for $\varepsilon>\varepsilon_{F}$. The number of quantum states in a volume $V$ available for electrons with momentum between $p$ and $p+d p$ is

$$
2 \frac{4 \pi p^{2} d p V}{(2 \pi \hbar)^{3}}
$$

Therefore the total number of electrons occupying states up to $p_{F}\left(\varepsilon=p^{2} / 2 m_{e}\right)$ is 


$$
N_{\iota}=\int_{0}^{p_{F}} 2 \frac{4 \pi p^{2} d p V}{(2 \pi \hbar)^{3}}=V \int_{0}^{\varepsilon_{F}} \frac{\varepsilon^{1 / 2}}{2 \pi^{2}}\left(\frac{2 m_{e}}{\hbar^{2}}\right)^{\frac{3}{2}} d \varepsilon=V \int_{0}^{\varepsilon_{F}} D(\varepsilon) d \varepsilon,
$$

where $D(\varepsilon)$ is called the density of states. Performing simple integration, we obtain the Fermi energy:

$$
\varepsilon_{F}=\frac{\hbar^{2}}{2 m_{e}}\left(3 \pi^{2} n_{e}\right)^{2 / 3}
$$

According to this formula, the Fermi energy in solid metals is approximately $5-10 \mathrm{eV}$ (see Table $2.1, \mathrm{p} .26$ ). This corresponds to a very high temperature of tens of thousands of degrees. Thus for a significant range of temperatures, the approximation $k_{B} T<<\mu(0)=\varepsilon_{F}$ is valid.

Taking the effect of Fermi distribution into account, we can see that heat capacity of electrons must be a linear function of temperature because the effective number of electrons is $n_{c} k_{B} T / \varepsilon_{F}$. After calculating the thermal energy $U_{e}$

$$
U_{e}=\int_{0}^{\infty} \varepsilon D(\varepsilon) f(\varepsilon) d \varepsilon-\int_{0}^{\varepsilon_{F}} \varepsilon D(\varepsilon) d \varepsilon
$$

the expression for heat capacity is obtained in the $k_{B} T<<\varepsilon_{F}$ limit

$$
C_{V e}=\gamma T=\frac{m_{e}}{\hbar^{2}}\left(\frac{\pi}{3}\right)^{\frac{2}{3}} n_{e}^{-\frac{1}{3}} k_{B} T=\frac{1}{3} \pi^{2} n_{e} \frac{k_{B} T}{T_{F}}
$$

with $\frac{3}{2} k_{B} T_{F}=\varepsilon_{F}$. Coefficient $\gamma$ has a typical value of $10^{-4} \mathrm{~J} /\left(\mathrm{cm}^{3} \mathrm{~K}^{2}\right)$ (see Table 2.1). Data in the table shows that the degenerate gas model gives results quite close to the measurements. 
Thermal response of the ions at low temperature is best described in terms of the collective lattice vibrations - elastic waves or phonons (quasiparticles carrying a quantum of vibrational energy). The internal energy $U_{l}$ can be calculated as

$$
U_{I}=\int_{0}^{\infty} \hbar \omega D(\omega) f(\omega) d \omega,
$$

where $\hbar \omega$ is the phonon energy, $D(\omega)$ is the density of phonon states and $f(\omega)$ is the Bose-Einstein distribution function [Landau69]

$$
f(\omega)=\frac{1}{e^{\hbar \omega / k_{B} T}-1} .
$$

The density of states is material specific and can be quite complicated, however the Debye model [Kitte196,Landau69,Mott58,Ziman60] offers a simple way to estimate the lattice heat capacity of a metal.

The main assumption of the model is linear frequency dependence on wavenumber for sound waves of all types (polarizations):

$$
\omega=c_{s} k
$$

where $c_{s}$ is the sound speed. The model accounts only for acoustic phonons. General formula for number of phonon states with wavevector between $k$ and $k+d k$ [Kittel96] is

$$
\frac{4 \pi k^{2} d k}{(2 \pi)^{3}}=\frac{4 \pi k^{2} d \omega}{(2 \pi)^{3}} \frac{d k}{d \omega}
$$

which gives a simple expression for the density of states

$$
D(\omega)=\frac{\omega^{2}}{2 \pi^{2} c_{s}^{3}}
$$


Since the lattice is a discrete system, there is a lower limit for a phonon wavelength supported by the material. This sets a frequency cut off, called the Debye frequency $\omega_{D}$. Coupling this with the fact that a finite collection of atoms can support only a finite number of phonon modes $n_{l}$, we can write an equation for the Debye frequency:

$$
\begin{gathered}
n_{l}=\int_{0}^{\omega_{D}} D(\omega) d \omega=\frac{\omega_{D}^{3}}{6 \pi^{2} c_{s}^{3}}, \\
\omega_{D}=c_{s}\left(6 \pi^{2} n_{l}\right)^{1 / 3} .
\end{gathered}
$$

With these assumptions the thermal energy for all polarizations (two transverse and one longitudinal) is given by

$$
U_{i}=3 \int_{0}^{\omega_{D}} \hbar \omega \frac{\omega^{2}}{2 \pi^{2} c_{s}^{3}} \frac{d \omega}{\exp \left(\hbar \omega / k_{B} T\right)-1} .
$$

The number of excited phonons carrying this energy is

$$
n_{p h}=3 \int_{0}^{\omega_{D}} \frac{\omega^{2}}{2 \pi^{2} c_{s}^{3}} \frac{d \omega}{\exp \left(\hbar \omega / k_{B} T\right)-1} .
$$

The heat capacity is the derivative of the energy integral

$$
C_{V l}=\int_{0}^{\omega_{B}} \frac{3(\hbar \omega)^{2}}{k_{B} T^{2}} \frac{\omega^{2}}{2 \pi^{2} c_{s}^{3}} \frac{\exp \left(\hbar \omega / k_{B} T\right) d \omega}{\left(\exp \left(\hbar \omega / k_{B} T\right)-1\right)^{2}} .
$$

This expression can be simplified to

$$
C_{V l}=3 n_{l} k_{b} \int_{0}^{1} \frac{3 x^{4} \exp (x / \alpha) d x}{\alpha^{2}(\exp (x / \alpha)-1)^{2}}=3 n_{l} k_{b} G(\alpha),
$$

with $x=\omega / \omega_{D}$ and $\alpha$ is dimensionless parameter $k_{B} T / \hbar \omega_{D}=T / \theta_{D}$, where $\theta_{D}$ is called the 
Debye temperature. In the two extreme cases of large and small $\alpha$, the integral can be simplified and easily calculated [Kittel96]. For high temperatures, $T>>\theta_{D}$, the heat capacity approaches $3 n_{i} k_{B}$ - the classical value of the heat capacity for $n_{l}$ oscillators. In the opposite case, $C_{V I}$ approaches zero proportionally to $T^{3}$. In order to understand which case is relevant to our problem, we need to calculate Debye temperature $\theta_{D}$. Using formula (2.13) with typical sound speed $c_{s} \sim 4 \cdot 10^{4} \mathrm{~cm} / \mathrm{s}$ and ion density $n_{t} \sim 7 \cdot 10^{22} \mathrm{~cm}^{-3}$ we obtain

$$
\theta_{D}=\frac{\hbar \omega_{D}}{k_{B}}=\frac{\hbar}{k_{B}} c_{s}\left(6 \pi^{2} n_{l}\right)^{1 / 3} \approx 500 \mathrm{~K}
$$

Data in Table 2.1 show that this number is close to actual material parameters.

Figure 2.2 presents the phonon heat capacity calculated for all values of $\alpha$. Since room temperature is approximately $300 \mathrm{~K} \sim \theta_{D}$, we see that for our problem the phonon heat capacity can be safely assumed to be equal to the classical value $3 n_{l} k_{B}$. Because the ion density is practically the same for many metals this number is approximately equal to $3 \mathrm{~J} /\left(\mathrm{cm}^{3} \mathrm{~K}\right)$

Combining expressions for electron and phonon heat capacities we get

$$
C_{V}=C_{V e}+C_{V I}=\frac{\pi^{2}}{3} Z n_{t} k_{B} \frac{T}{T_{F}}+3 n_{l} k_{B} \approx 10^{-4} \frac{\mathrm{J}}{\mathrm{cm}^{3} \mathrm{~K}^{2}} T+3 \frac{\mathrm{J}}{\mathrm{cm}^{3} \mathrm{~K}^{2}} .
$$

For temperatures up to thousands of degrees, the heat capacity of a metal is dominated by the lattice(ions) - opposite to the ideal plasma case. 


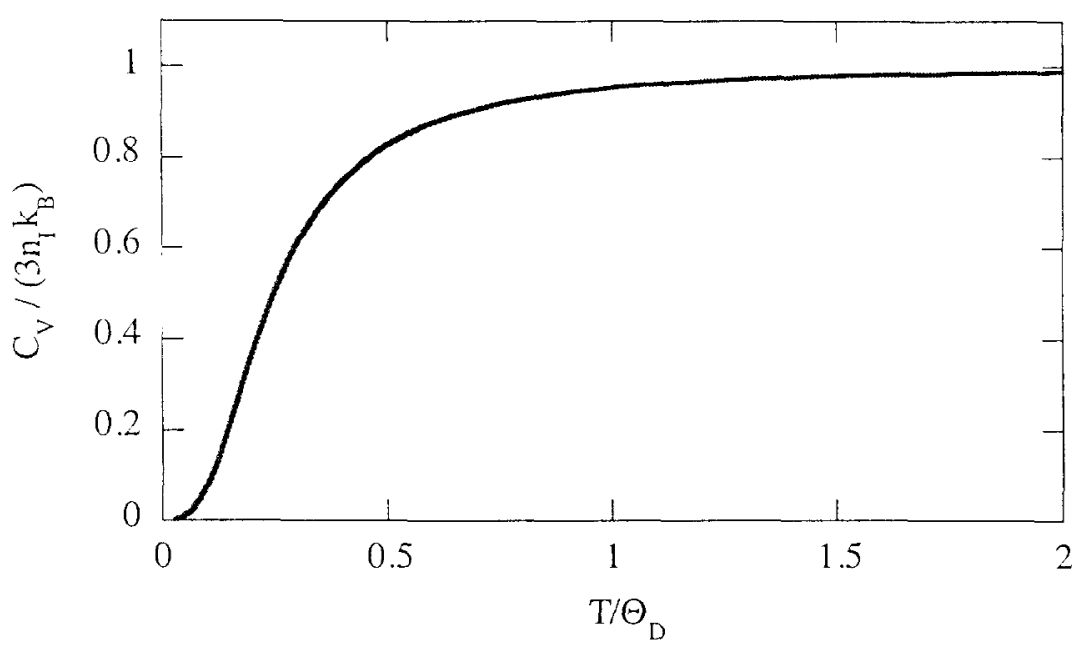

Figure 2.2: Phonon heat capacity as a function of temperature according to the Debye model. High temperature limit is $3 n_{I} k_{B}$.

Using the above results we can make a simple estimate of temperatures that can be reached in USLP absorption. Assuming that the laser pulse has fluence $F$ (energy per unit area) and a fraction $A$ of this energy is absorbed in a layer with thickness $l$, the peak temperature is

$$
T_{p}=\frac{A F}{l C_{V}} .
$$

Using absorption of $10 \%$ in a layer $20 \mathrm{~nm}$ thick and heat capacity of $3 \mathrm{~J} /\left(\mathrm{cm}^{3} \mathrm{~K}\right)$, we arrive at

$$
T_{p}[\mathrm{~K}] \approx 2 \cdot 10^{5} F\left[\mathrm{~J} / \mathrm{cm}^{2}\right], \quad T_{p}[\mathrm{eV}] \approx 2 F\left[\mathrm{~J} / \mathrm{cm}^{2}\right]
$$

According to this estimate, material damage (heating to a few thousand degrees material melting and boiling) will appear for a fluence of tens of millijoules per square 
centimeter. Moderate fluences of several joules per square centimeter will produce hot plasma with temperature of several electronvolts.

\begin{tabular}{|c|c|c|c|}
\hline METAL & $\mathrm{Al}$ & $\mathrm{Cu}$ & $\mathrm{Au}$ \\
\hline Atomic number $\mathrm{A}$ & 27 & 63.5 & 197 \\
\hline Density $\rho, \mathrm{g} / \mathrm{cm}^{3}$ & 2.7 & 8.93 & 19.3 \\
\hline Thermal conductivity $\chi, \mathrm{W} / \mathrm{cmK}$ & 2.38 & 4.0 & 3.11 \\
\hline \multicolumn{4}{|l|}{ ELECTRONIC PROPERTIES } \\
\hline Electron density $n_{e}, \times 10^{23} \mathrm{~cm}^{-3}$ & 1.8 & 0.845 & 0.59 \\
\hline Calculated Fermi energy $\varepsilon_{r}, \mathrm{eV}$ & 11.63 & 7.0 & 5.51 \\
\hline Calculated $T_{F}=2 \varepsilon_{F} / 3 k_{B}, \times 10^{4} \mathrm{~K}$ & 9.0 & 5.4 & 4.26 \\
\hline Calculated $\gamma, \times 10^{-4} \mathrm{~J} /\left(\mathrm{cm}^{3} \mathrm{~K}^{2}\right)$ & 0.912 & 0.71 & 0.63 \\
\hline Measured $\gamma, \times 10^{-4} \mathrm{~J} /\left(\mathrm{cm}^{3} \mathrm{~K}^{2}\right)$ & 1.35 & 0.98 & 0.71 \\
\hline Calculated $u_{F}=\sqrt{2 \varepsilon_{F} / m_{e}}, \times 10^{8} \mathrm{~cm} / \mathrm{s}$ & 2.02 & 1.57 & 1.39 \\
\hline \multicolumn{4}{|l|}{ LATTICE PROPERTIES } \\
\hline Ion density $n_{l}, \times 10^{22} \mathrm{~cm}^{-3}$ & 6 & 8.45 & 5.9 \\
\hline Debye temperature $\theta_{D}, \mathrm{~K}$ & 428 & 343 & 165 \\
\hline Longitudinal sound speed $c_{s}, \times 10^{5} \mathrm{~cm} / \mathrm{s}$ & 6.4 & 5.0 & 3.24 \\
\hline Heat capacity $C_{p} \approx C_{v}, \mathrm{~J} /\left(\mathrm{cm}^{3} \mathrm{~K}\right)$ & 2.45 & 3.46 & 2.51 \\
\hline Calculated $C_{V}=3 n, k_{B}, \mathrm{~J} /\left(\mathrm{cm}^{3} \mathrm{~K}\right)$ & 2.5 & 3.5 & 2.44 \\
\hline
\end{tabular}

Table 2.1: Experimental [Kittel96,Gray63] and calculated electronic and lattice properties of several NFE metals. Electronic properties are calculated with degenerate gas model and lattice properties are calculated using Debye model [Kittel96]. All data is at room temperature

The simple formula (2.21) gives us an upper limit. In reality, the peak temperature may be lower and one of the main processes responsible for this is thermal diffusion that 
transports absorbed energy away from the surface into the bulk of the material. Metals, especially NFE metals, are very good conductors. The same is true for plasmas.

To see what particles are responsible for thermal conduction and how significant this effect might be, let's consider a simple kinetic theory [Kitte196]. Suppose we have a temperature gradient. There is a constant movement of particles along the gradient in each direction. The net particle flow must be zero because of mass conservation. However particles moving from the region with higher temperature carry more energy, which is then transferred to colder particles in collisions. As a result, there is a net flow of energy along the temperature gradient. This flow can be estimated as

$$
j \approx-n u c \delta T
$$

where $n$ is particle density, $c$ is heat capacity per particle, $u$ is average particle velocity and $\delta T$ is a temperature difference on one mean free path length $\lambda$. Rewriting this temperature difference as $\delta T=\lambda \nabla T$, energy flow becomes

$$
j \approx-n u c \lambda \nabla T .
$$

From this expression we can see that thermal conduction coefficient $\chi \sim c n u \lambda$. Formal kinetic theory gives qualitatively the same answer [Lifshitz81]

$$
\chi=\frac{1}{3} C u \lambda=\frac{1}{3} C u^{2} \tau
$$

where $C$ is heat capacity and $\tau=\lambda / u$ is collision time.

In the case of plasma, we can expect heat capacity and collision time have similar 
magnitude for ions and electrons, since these parameters are essentially determined by similar particle densities and temperatures. The most significant factor is particle velocity $u=3 k_{i} T / 2 m$. Since atomic number $A$ is ten or higher, a typical metal ion is at least ten thousand times heavier than the electron

$$
\frac{m_{e}}{m_{l}}=\frac{m_{e}}{A m_{p}}=\frac{5.4 \cdot 10^{-4}}{A} .
$$

As a result, the velocity of electrons is much larger and thermal transport in plasma is completely dominated by electrons.

Formula (2.24) is also valid for degenerate electrons and phonons [Ziman60]. According to equation (2.19), the heat capacity of electrons at moderate temperature is one-two orders of magnitude smaller than the heat capacity of phonons. However, the average velocity of electrons $u_{F}=\sqrt{2 \varepsilon_{F} / m_{e}} \approx 1.5 \cdot 10^{8} \mathrm{~cm} / \mathrm{s}$ is about two-three orders of magnitude larger than the phonon velocity - sound speed $c_{s} \sim 4 \cdot 10^{5} \mathrm{~cm} / \mathrm{s}$ (see Table 2.1). Ziman [Ziman60] estimates that the mean free path of electrons in a metal is larger than the mean free path of a phonon by a factor as large as ten. Thus, electrons also dominate thermal diffusion in metals.

If heat capacity and thermal conductivity coefficients are known, we can estimate the penetration depth of the thermal wave generated by laser absorption and propagating into the bulk of the material. Since metals absorb laser energy in a very thin surface layer, this problem is similar to the problem of a point source. According to the dimensional 
considerations [Sedov93], the temperature depends on distance as $T\left(x / x^{*}\right)$, where $x^{*}$ is a characteristic length that must be constructed from available parameters - heat capacity, conductivity coefficient, time and deposited energy. The only way to do this is to set it to

$$
x^{*}=\sqrt{\frac{\chi}{C} t^{*}}
$$

where $t^{*}$ is propagation time. Parameter $x^{*}$ is called the thermal diffusion length. For metals heat capacity is $C \sim 3 \mathrm{~J} /\left(\mathrm{cm}^{3} \mathrm{~K}\right)$ (dominated by phonons) and thermal conductivity is $\chi \sim 3 \mathrm{~W} /(\mathrm{cmK})$ (dominated by electrons). If the pulse length is below $1 \mathrm{ps}$, this distance is within $10 \mathrm{~nm}$ - comparable to the skin depth of the material. Therefore, for the case when electron and phonon temperatures are equal, thermal diffusion is not a significant factor.

In the next part of this chapter we consider the validity of this approximation and present modeling of dynamics of electron and phonon temperatures.

\subsection{Two temperature model of laser energy absorption}

Laser energy is deposited in electrons; however the previous discussions assumed that electrons and ions/phonons are in thermal equilibrium. For this assumption to be valid, the temperature equilibration time must be much smaller than the characteristic time of the laser material interaction. Energy exchange between particles occurs through collisions, thus the equilibration rate depends on collision frequency $v$ (collision time $\tau=1 / v)$ and fraction of energy $\delta$ transferred in each collision. Modeling of the collision 
frequency will be discussed in the next section. However, we can understand the process of equilibration without going into details of calculation of $v$.

In order to do that, lets review a simple form of energy exchange act: a fast moving particle (electron) collides with a particle at rest (ion) or excites a quasiparticle (phonon).

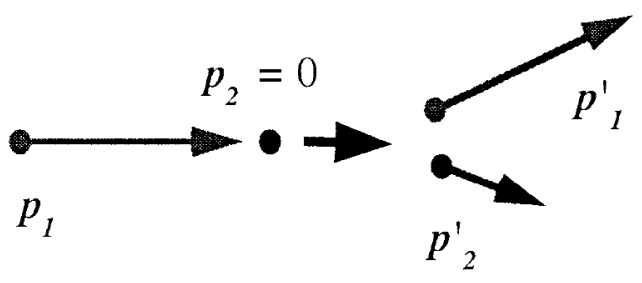

Figure 2.3: Schematic diagram of particle 1 (momentum $p_{l}$ ) colliding with particle 2 (momentum $p_{2}=0$ ).

Energy and momentum conservation laws dictate that

$$
\left\{\begin{array}{l}
E_{1}=E_{1}^{\prime}+E_{2}^{\prime} \\
p_{1}=p_{1}^{\prime}+p_{2}^{\prime}
\end{array}\right.
$$

where $E_{l}(p)$ and $E_{2}(p)$ are energy dispersion laws for the incident and resting particles respectively. We need to estimate the fraction of the energy transferred from particle 1 to particle 2 after the collision

$$
\delta=\frac{E_{2}^{\prime}}{E_{1}}=\frac{E_{2}\left(p_{2}^{\prime}\right)}{E_{1}\left(p_{1}\right)} .
$$

Particles of interest have energy monotonically increasing as a function of momentum magnitude. In other words, the larger the momentum modulus $|p|$, the larger the energy. 
Zero momentum means zero energy. Therefore we can say that out of all possible scenarios for the collision the largest momentum particle 2 can acquire is $2\left|p_{1}\right|$. Indeed, according to the conservation equation, the final energy $E_{1}^{\prime}$ of particle 1 is less than or equal to its initial energy, thus the magnitude of the final momentum $\left|p_{l}^{\prime}\right|$ is less than or equal to $\left|p_{1}\right|$. Subtraction of the two momentum vectors always produces a vector with magnitude less or equal to the sum of moduli of the two original vectors

$$
\left|p_{2}^{\prime}\right|=\sqrt{\left(p_{1}-p_{1}^{\prime}\right)^{2}}=\sqrt{\left|p_{1}\right|^{2}+\left|p_{1}^{\prime}\right|^{2}+2\left|p_{1}\right|\left|p_{1}^{\prime}\right| \cos \left(p_{1}, p_{1}^{\prime}\right)} \leq\left|p_{1}\right|+\left|p_{1}^{\prime}\right| \leq 2\left|p_{1}\right| .
$$

As a result the fraction of energy transferred is

$$
\delta \leq \delta_{m}=\frac{E_{2}\left(2 p_{1}\right)}{E_{1}\left(p_{1}\right)} .
$$

In case of electron-ion collisions, the energy dispersion is $p^{2} / 2 m_{e}$ for electrons and $p^{2} / 2 m_{l}$ for ions. The maximum energy transfer is then

$$
\delta_{m}=4 \frac{m_{e}}{m_{l}}
$$

In case of electron-phonon collisions, the energy dispersion is $p^{2} / 2 m_{e}$ for electrons and $p c_{s}$ for phonons. Maximum energy transfer is then

$$
\delta_{m}=4 \frac{c_{s}}{u_{F}} .
$$

According to both of these formulas, the fraction of the energy transferred from an electron to an atom in a plasma or a solid is $10^{-3}-10^{-5}$ (see formula (2.25) and Table 2.1 for needed data). At the same time, for collisions of similar particles we have $\delta_{m} \sim 1$. 
Therefore, equilibrium between identical particles is reached much faster than between particles of different types.

If we estimate the electron collision time in a solid as the time it takes for electrons to travel from one atom to another

$$
\tau \sim \lambda / u_{F} \sim \frac{3 \cdot 10^{-8} \mathrm{~cm}}{3 \cdot 10^{8} \mathrm{~cm} / \mathrm{s}} \sim 10^{-16} \mathrm{~s}
$$

and take $\delta_{m}$ to be $10^{-5}$, temperature equilibration time between phonons and electrons becomes equal to $10 \mathrm{ps}$. Since the collision time estimate gives us the smallest possible number, we can certainly say that during USLP absorption electrons and phonons/ions are not in the equilibrium.

The collision time in plasmas can be much larger than in the estimate above, thus the non-equilibrium state between ions and electrons may exist for a long time [Spitzer62]. In the case of solids, the possibility of non-equilibrium conditions first attracted attention in connection with electron emission from metals under the action of powerful electric currents or laser pulses[Ginsburg55,Anisimov74]. Thermal transport in both plasmas and solids can be described by a two temperature model - temperature of electrons and ions/phonons is modeled by a system of regular thermal diffusion equations for each particle type with an energy exchange term added [Spitzer62,Anisimov74]

$$
\left\{\begin{array}{l}
C_{v_{e}} \frac{d T_{e}}{d t}=\nabla \cdot \chi_{e} \nabla T_{e}+G_{e}\left(T_{e}, T_{l}\right)+S_{e}(r, t) \\
C_{V I} \frac{d T_{l}}{d t}=\nabla \cdot \chi_{l} \nabla T_{I}+G_{I}\left(T_{e}, T_{l}\right)+S_{I}(r, t)
\end{array},\right.
$$


where $\nabla \chi \bullet \nabla T$ describes the effect of thermal diffusion, $G\left(T_{e}, T_{l}\right)$ is the energy exchange term and $S(r, t)$ represents other sources and sinks like laser energy absorption or shock wave heating. The energy exchange term can be rewritten in a clearer form. Because of energy conservation, we have $G_{e}\left(T_{e}, T_{l}\right)=-G_{l}\left(T_{v}, T_{l}\right)$. When electrons and ions are in equilibrium, this function must be zero. To illustrate this it can be rewritten as

$$
G_{e}\left(T_{e}, T_{l}\right)=g\left(T_{e}, T_{I}\right)\left(T_{l}-T_{e}\right) .
$$

When electron and ion temperatures are equal, the energy exchange term vanishes by definition and the two equations can be replaced by one with heat capacity and conductivity coefficients equal to the sum of the respective electron and ion/phonon parts.

Intuitively, it is clear that the function $g\left(T_{e}, T_{l}\right)$ depends on the collision frequency $v$ and the fraction of energy transferred $\delta$ - the higher $v$ and $\delta$ are, the larger the energy exchange rate is. The exact relationship between these parameters is material and temperature specific. However in the case of a low temperature solid metal, the function $g\left(T_{e}, T_{i}\right)$ is known to be simply a constant [Kaganov57]

$$
g\left(T_{e}, T_{l}\right)=\frac{m_{e}^{2} U^{2}\left(k_{B} \theta_{D}\right)^{5}}{2 \hbar^{7} \rho s^{4}(2 \pi)^{3} \theta_{D}} \sim \frac{m_{e}^{2} U^{2} k_{B} n_{l}^{4 / 3}}{\hbar^{3} \rho}=\frac{m_{e}}{m_{l}} k_{B} \hbar^{-3} m_{e} U n_{l}^{1 / 3}
$$

where $U$ is an electron-lattice interaction constant. Parameter $g$ is inversely proportional to the material density. Since ion density $n_{l}$ is similar for all metals, it effectively means 
that energy exchange is slower for heavier ions - consistent with our previous conclusions.

Based on this fact, a very simple two temperature model was suggested for description of ultrashort laser interaction with metals [Anisimov74,Anisimov97]

$$
\left\{\begin{array}{l}
C_{V_{e}} \frac{d T_{e}}{d t}=\frac{d}{d z}\left(\chi \frac{d T_{e}}{d z}\right)+g\left(T_{l}-T_{e}\right)+S(z, t) \\
C_{V l} \frac{d T_{l}}{d t}=g\left(T_{e}-T_{l}\right)
\end{array}\right.
$$

Since heat is carried mostly by electrons, the thermal diffusion term for the lattice is omitted. The typical laser spot is much larger than the heat penetration depth, therefore the equations are one dimensional. The constant $g$ can be measured experimentally. Function $S(z, t)$ describes laser energy deposition.

If energy exchange is slower than energy deposition and thermal transport, the system of equations (2.33) can be considered decoupled. This has an important effect on the heat wave propagation. If one calculates the diffusion length according to (2.26), it must be done using the heat capacity of electrons! Since it is two orders of magnitude smaller than the heat capacity of the lattice, thermal wave will propagate $100 \mathrm{~nm}$ after $1 \mathrm{ps}$, much larger than our original estimate of $10 \mathrm{~nm}$ for the case of equal temperatures. The actual result, of course, lies somewhere between these two extreme estimates. Our analysis shows that thermal transport can be neglected only for very short pulses. 
In order to see how significant the difference between electron and lattice temperatures can be, we solve the system of equations (2.33) after some simplifications. The only term that explicitly makes it a system of partial differential equations is electron thermal transport. If we consider a very short $100 \mathrm{fs}$ pulse, the maximum diffusion length is approximately $30 \mathrm{~nm}$. Since this is an upper estimate and this number is not much larger than an optical energy deposition depth of $10 \mathrm{~nm}$, we can model the temperature response to a rectangular laser pulse $100 \mathrm{fs}$ long assuming that energy is absorbed uniformily in a thin surface layer. Thus the thermal transport term is not needed and we obtain a system of ordinary differential equations

$$
\left\{\begin{array}{l}
C_{V e} \frac{d T_{e}}{d t}=g\left(T_{l}-T_{e}\right)+\frac{A I}{l} \\
C_{V I} \frac{d T_{l}}{d t}=g\left(T_{e}-T_{l}\right)
\end{array},\right.
$$

where $A$ is absorptivity of the metal for given laser parameters, $I$ is the laser intensity and $l$ is the energy deposition depth that we set to $20 \mathrm{~nm}$. Once heat capacities are defined, the system can be easily solved numerically in Mathematica [Wolfram99].

Heat capacities of the NFE metal can be approximated as

$$
\left\{\begin{array}{l}
C_{V e}\left(T_{e}<T^{*}\right)=\gamma T_{e} ; \quad C_{V e}\left(T_{e}>T^{*}\right)=\frac{3}{2} N_{e} k_{B}, \quad T^{*}=\frac{3 N_{e} k_{B}}{2 \gamma} \\
C_{V I}\left(T_{l}<T_{m}\right)=3 N_{l} k_{B} ; \quad C_{V I}\left(T_{I}>T_{m}\right)=\frac{3}{2} N_{I} k_{B}\left(1+T_{m} / T_{I}\right)
\end{array},\right.
$$

where $T_{m}$ is the melting temperature. The formulas are simple interpolations between the different temperature regimes discussed in the previous section. 
Calculations were performed for $800 \mathrm{~nm}$ laser light for aluminum and copper. Absorptivity is set to $0.13(\mathrm{Al})$ and $0.037(\mathrm{Cu})$ - absorption of $800 \mathrm{~nm}$ laser light at normal incidence [Palik98], the energy exchange coefficient is set to $2 \cdot 10^{11} \mathrm{~W} /\left(\mathrm{cm}^{3} \mathrm{~K}\right)$ for aluminum [Eidmann00] and to $10^{11} \mathrm{~W} /\left(\mathrm{cm}^{3} \mathrm{~K}\right)$ for copper [Elsayed-Ali87]. The melting temperature is $930 \mathrm{~K}$ for aluminum and $1790 \mathrm{~K}$ for copper - isochoric melting happens at a slightly higher temperatures than isobaric melting[Steinberg91]. All other needed parameters can be found in Table 2.1.

Figure 2.4 shows the calculated temporal evolution of electron and lattice temperatures for a $10^{12} \mathrm{~W} / \mathrm{cm}^{2}$ laser pulse.

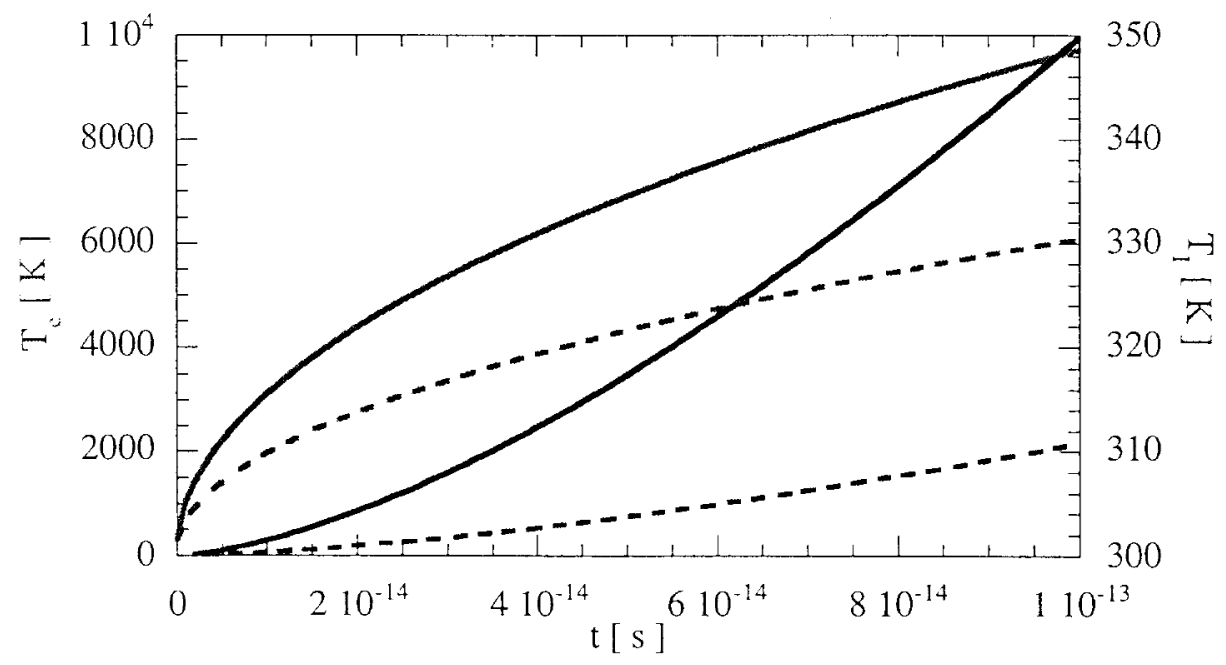

Figure 2.4: Temporal evolution of the electron (red lines, left axis) and lattice (blue lines, right axis) temperature of aluminum (solid lines) and copper (dashed lines) absorbing $800 \mathrm{~nm}$ laser light with intensity $10^{12} \mathrm{~W} / \mathrm{cm}^{2}$. Calculations are done according to the system of equations (2.34).

We can immediately see the significant disparity between these two temperatures. The 
electron heat capacity of a cold metal is linearly proportional to temperature and has a small value. As result, temperature grows initially very fast, as the square root of time, and almost instantaneously jumps to thousands degrees. The electron temperature in aluminum increases faster, mostly because of the higher laser absorption, and by the end of the pulse almost reaches $1 \mathrm{eV}$. At the same time, the lattice remains practically cold. Even for aluminum with its hotter electrons and higher energy exchange rate, the ion temperature increase at the pulse end is less than a hundred degrees.

Figure 2.5 presents temperatures at the pulse end as a function of laser intensity.

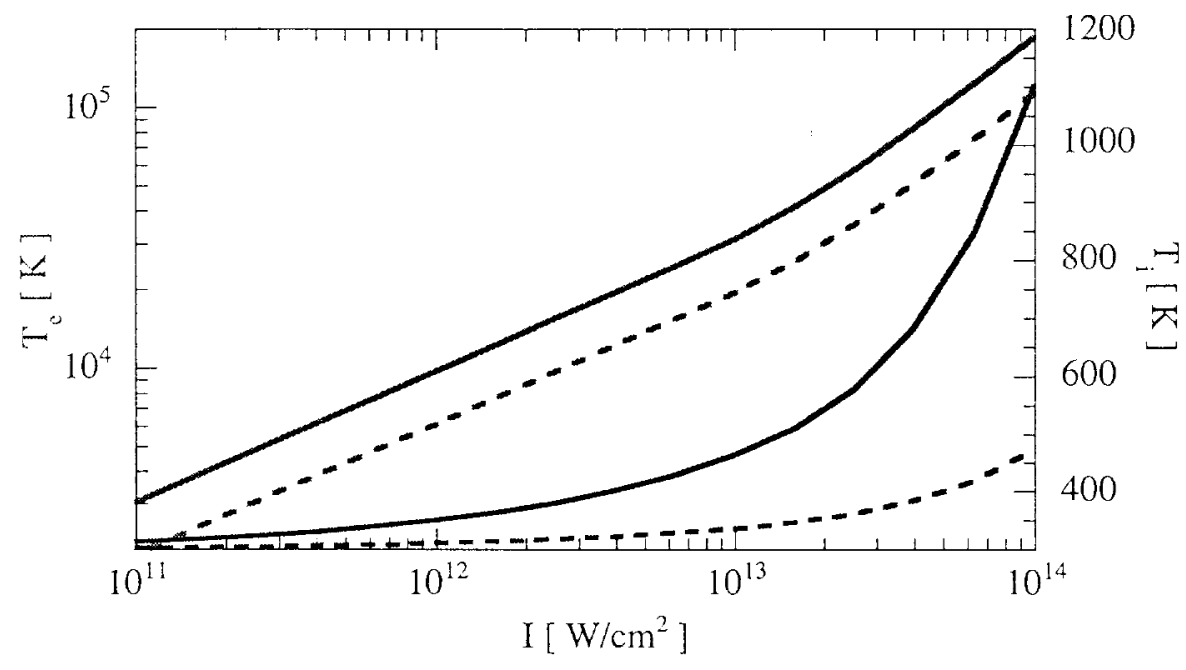

Figure 2.5: Electron and lattice temperature of aluminum (solid lines) and copper (dashed lines) at the end of rectangular $100 \mathrm{fs} 800 \mathrm{~nm}$ laser pulse as a function of light intensity. Calculations are done according to the system of equations (2.34).

The graphs shows a similar picture: there are orders of magnitude difference between electron and phonon temperatures. If electrons and ions were in equilibrium, a laser pulse 
with intensity $10^{14} \mathrm{~W} / \mathrm{cm}^{2}$ would have enough energy (pulse fluence $=10 \mathrm{~J} / \mathrm{cm}^{2}$ ) to turn the surface layer into hot plasma. However the energy exchange rate is not fast enough to do it during the pulse and at the end the lattice temperature is barely above the melting temperature.

This modeling represents an extreme case, which is applicable only to a relatively low temperature. If temperature is very high (many $\mathrm{eV}$ ) material begins to transform into plasma, where the function $g$ is no loner a constant. Also it is more common to work with Gaussian pulses, therefore there is more time for lattice heating and energy diffusion. Nonetheless, it demonstrates that during laser absorption electrons are significantly out of equilibrium with the lattice, which may be melted only after the pulse is over. Typical lattice heating time can be estimated as $C_{V l} / g$. This gives $12 \mathrm{ps}$ for aluminum and $35 \mathrm{ps}$ for copper. Higher density materials like gold have even slower energy exchange rates and need an even larger time. Since material ablation requires removal of ions, there can be a significant delay between pulse absorption and actual ablation, when hot particles leave en masse. However true electron-ion equilibration time is difficult to calculate. Lattice heating time gives an upper estimate. If electron temperature is noticeably smaller than the Fermi temperature, this time is really defined by the electron heat capacity: $\tau_{c q}=C_{V e} / g$ and has a value on the order of hundreds of femtoseconds. The process is further complicated by the continuing electron thermal transport of the laser energy away 
from the surface to the bulk of the material. Therefore numerical simulations based on non-equilibrium treatment of electrons and ions must be a preferred way to model ultrashort laser-material interaction.

\subsection{Collision frequency}

The subject of collisions was brought up several times in our discussions of thermal properties. Since energy and momentum exchange between particles happens through collisions, they play an important role in the dynamics of equilibration. For instance, a high rate of collisions is an indication of fast approach to local thermal equilibrium. On the other hand, faster thermal transport would require a decrease in collision frequency. This section is devoted to a review of this important phenomena.

Full treatment of collisions is based on solution of kinetic equations together with a proper description of the physics of particle interaction [Lifshitz81]. However qualitative results can be obtained using a simple expression for the collision time $\tau$. Assume we have a gas of hard particles moving with average speed $u$. When a particle travels distance $\lambda$ between collisions, it covers a volume $\sigma \lambda$, where $\sigma$ is the particle crosssectional area. There is only one particle in this volume, therefore $n \sigma \lambda=1$ and the expression for collision time immediately follows

$$
\tau=\frac{\lambda}{u}=\frac{1}{n \sigma i}
$$

In a more general situation, the parameter $\sigma$ is an effective collision cross-section that 
depends on details of interparticle interaction and is a function of material properties.

For some cases, for example a monatomic gas, the collision cross-section is indeed a constant, and the trivial application of (2.35) to calculation of, for instance, the thermal conductivity coefficient (see (2.24)) gives us

$$
\chi=\frac{1}{3} C u \lambda=\frac{1}{2} k_{B} n u \frac{1}{n \sigma} \sim u \sim \sqrt{T} .
$$

Energy diffusion in an ideal gas is only a function of temperature, but not density or pressure.

Treatment of plasmas is more complicated, since charged particles interact through long-range Coulomb forces and can affect each other at any distance. Therefore there is no clearly defined collision. However, an effective cross-section $\sigma$ can be introduced if we assume that a collision is an event in the which particle, in the process of interaction, significantly changes its direction. This happens if the change of momentum $\delta p$ induced by the interaction force $F$ is comparable to the initial particle momentum $p=m u$. The typical interaction time $t^{*}$ can be estimated as $b / u$, where $b$ is the distance of the closest approach if there were no potential interaction. Combining everything together we get

$$
\frac{\delta p}{p} \sim \frac{F t^{*}}{m u} \sim \frac{\left(Z_{1} Z_{2} e^{2} / b^{2}\right)(b / u)}{m u}=\frac{Z_{1} Z_{2} e^{2}}{b m t^{2}} \sim 1
$$

where the force was estimated as $Z_{1} Z_{2} e^{2} / b^{2}$ and $e Z_{1}, e Z_{2}$ are particle charges. In this approach, the collision cross-section $\sigma$ is $\pi b^{2}$, therefore the collision time is 


$$
\tau \sim \frac{m^{2} u^{3}}{n\left(Z_{1} Z_{2}\right)^{2} e^{4}} \sim \frac{\sqrt{m} T^{3 / 2}}{n\left(Z_{1} Z_{2}\right)^{2}}
$$

This is a somewhat counterintuitive result - collisions become less frequent when the temperature goes up and the particle velocity increases. The dominant term in this case is the collision cross-section, which decreases with temperature as $T^{-2}$, thus the reduction in collision frequency.

We should notice that formula (2.36) is the only way to obtain a parameter with the dimension of distance from the available variables, therefore one may expect to get a similar expression for collision time from kinetic theory. Indeed, the electron-ion collision time in a plasma is described by [Spitzer62,Lifshitz81,Artsimovich93]

$$
\tau_{e l}=\frac{3 \sqrt{m_{e}}\left(k_{B} T_{c}\right)^{3 / 2}}{4 \sqrt{2 \pi} n_{l} Z^{2} e^{4} \operatorname{Ln}(\Lambda)},
$$

where $Z$ is an average ionization level of an ion and $\operatorname{Ln}(\Lambda)$ is the so-called Coulomb logarithm, which is a slowly varying function of temperature and density. It describes modification of the potential interaction, for example due to the collective effects, and has a typical value of 10-20 [Spitzer62].

The same theory gives expressions for electron-electron and ion-ion collision times. Because they involve the same type of interaction, the formulas are qualitatively similar and differ only in magnitude. Due to the differences in mass, charge and density the following relationship holds between these parameters [Trubnikov65] 


$$
\tau_{e l}: \tau_{e e}: \tau_{I I} \sim 1: Z: \frac{1}{Z^{2}} \sqrt{\frac{m_{l}}{m_{e}}}
$$

Since in a typical metal, plasma ions are much heavier and multiply ionized (but not completely), the electron-ion collision time is the smallest. Thus, it dominates the total collision frequency $v=\sum v_{i}=\sum \tau_{i}^{-1}$

In plasma, electrons collide with ions even when the ions are at rest. This is not true for low temperature crystalline solids. From the quantum mechanical description it is known that electrons propagate through an ideal lattice (ions are fixed at an equally spaced locations) without experiencing collisions. The collisions come from interaction with lattice irregularities, for example point defects. In NFE metals, in the temperature regime we are interested in, the imperfections mostly come from thermal excitation of the lattice - phonons. Full treatment of the subject is quite complicated [Ziman60, Abrikosov72], however it is easy to understand where the major contribution comes from. According to (2.35) there are three important factors - collision cross-section, particle velocity and number of scattering particles. Since only electrons near the Fermi energy can scatter, particle velocity is practically a constant $u_{F}$. On the other hand, the number of phonons varies. For lattice temperatures $T_{P}>\theta_{D}$ the heat capacity of the phonon gas is constant, thus the total energy is a linear function of temperature. Since, in this regime each phonon carries fixed a amount of energy $\varepsilon \sim k_{B} \theta_{D}$, the total number of phonons must be proportional to the lattice temperature. Indeed, expansion of the integral 
(2.15) proves that

$$
n_{p h} \sim T_{I} / \theta_{D}
$$

The cross-section $\sigma$ describes details of the phonon-electron interaction and can be considered a constant [Ziman60,Abrikosov72]. As a result, the collision time is proportional to the inverse ion temperature

$$
\tau_{e-p h}=\tau_{0} \frac{\theta_{D}}{T_{l}}
$$

Similar behavior is observed for melted metals [Mott58], so the formula (2.40) can still be used to describe effect of melting, however the constant $\tau_{0}$ must be replaced by a different constant $\tau_{0 m}$, which is approximately two times smaller for metals like aluminum or copper [Mott58]. The exact value of these constants can be determined, for example, from our knowledge of the heat conductivity coefficient. For good conductors the collision time at room temperature is on the order of $10^{-14} \mathrm{~s}$.

Electron-electron collisions in a solid can be reviewed in a similar approach. Since particle velocity is a constant $u_{F}$, the collision cross-section is fixed and temperature dependence can appear only in the number of scattering particles. Pauli exclusion principle dictates that only a small fraction of electrons $n_{e} k_{B} T / \varepsilon_{F}$ can participate in the collisions. Because both electrons come from this group, there is another factor $k_{B} T_{f} / \varepsilon_{F}$. Therefore, the electron-electron collision time in solids is inversely proportional to temperature squared. Abrikosov [Abrikosov72] suggests the following estimate for the 
electron-electron collision time

$$
\tau_{e e} \sim \frac{\hbar}{\varepsilon_{F}}\left(\frac{\varepsilon_{F}}{k_{B} T_{e}}\right)^{2} .
$$

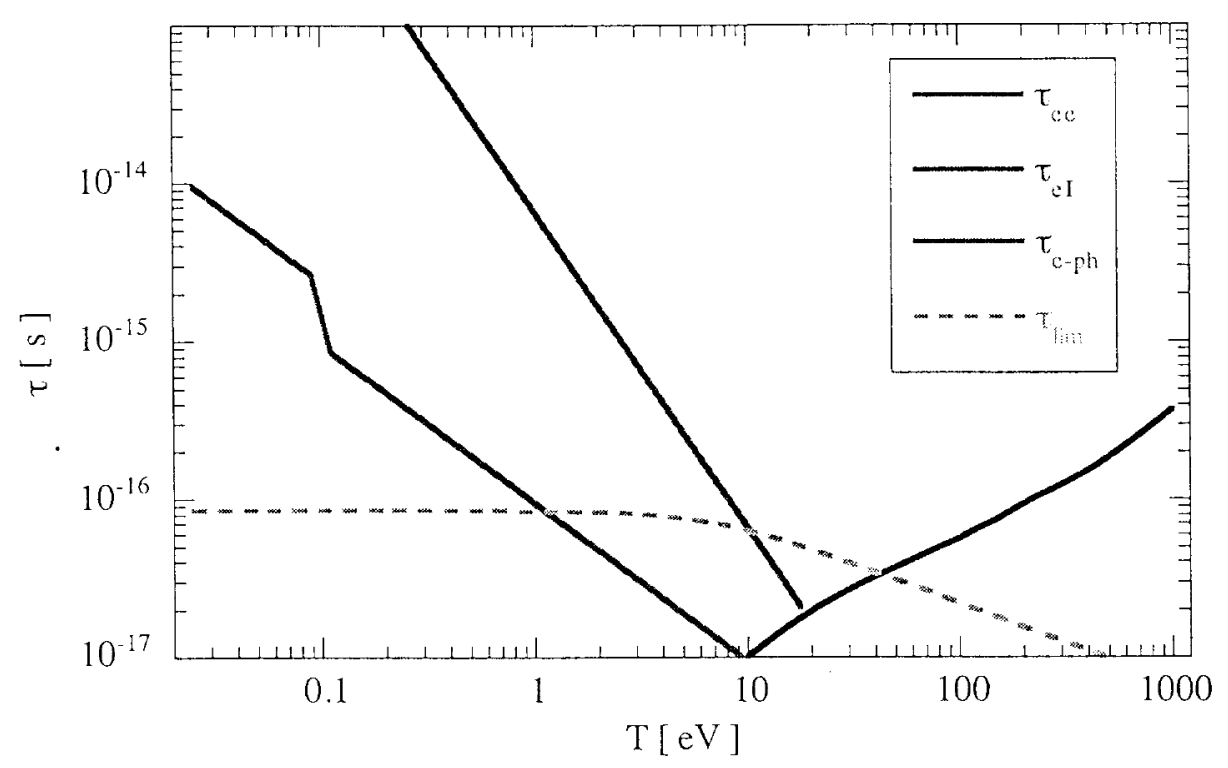

Figure 2.6: Different collision times in solid density aluminum as a function of material temperature.

According to this formula $\tau_{e e}$ is $10^{-11} \mathrm{~s}$ at room temperature. When electrons and the lattice are in equilibrium, electron-phonon collisions are dominant.

In case of intermediary temperatures when neither of the above models is truly valid, calculation of collision time is especially difficult. This is the regime of nonideal strongly coupled plasma, when kinetic and potential energy have similar values and simple models do not work. The collision time can be estimated to be no less than the time it take electron to travel from one atom to another 


$$
\tau_{\lim }=\frac{R}{u}=\frac{\left(3 / 4 \pi n_{l}\right)^{1 / 3}}{\sqrt{3 \frac{k_{B}}{m_{e}} \sqrt{T_{e}^{2}+T_{F}^{2}}}}
$$

For small temperatures, the particle velocity $u$ is equal to Fermi velocity $u_{F}$, at high temperatures it is equal to thermal velocity.

Figure 2.6 presents calculated collision times for solid density aluminum. The general behavior should be similar for all NFE metals - collision time get smaller with temperature increase, at around Fermi temperature it reaches a minimum on the order of $10^{-16} \mathrm{~s}$ and then begins to grow. At low temperatures, electron-phonon collisions completely dominate electron-electron collisions, however the latter depends on electron temperature, thus in case of strong equilibrium it can be important.

The subject of collisions plays a central role in the discussion of optical properties in the next section.

\subsection{Optical properties of metals and light absorption}

Interaction of laser light with matter is a very rich topic. Our primary goal is to model light absorption as a function of laser parameters and material state. Electromagnetic wave propagation is described by Maxwell's equations, where material reaction is expressed by permittivity $\varepsilon$ and conductivity $\sigma$ (which are typically combined in one complex dielectric function $\varepsilon$ ). To simulate USLP absorption we need a simple, usable model of dielectric function that captures the most significant aspects of 
absorption and reflection.

In NFE metals or plasmas response to a high frequency electromagnetic wave is driven by the behavior of electrons. If we can find expression for electric current $\vec{j}$ as a function of wave field $\vec{E}$, the formula for dielectric constant $\varepsilon$ follows from the field equation

$$
\nabla \times \vec{B}=\frac{4 \pi}{c} \vec{j}+\frac{1}{c} \frac{\partial \vec{E}}{\partial t}=\frac{1}{c} \frac{\partial(\varepsilon \vec{E})}{\partial t} .
$$

Writing electron current $\vec{j}$ as $\sigma \vec{E}$, for periodically oscillating field $\vec{E} \mathrm{e}^{-i \omega h}$ we obtain formula for dielectric function

$$
\varepsilon(\omega)=1+i \frac{4 \pi}{\omega} \sigma(\omega)
$$

Electric current can be approximated as $\vec{j}=e n_{e} u=e n_{e} d \vec{x} / d t$, thus conductivity $\sigma$ can be found through relationship between electron trajectory $\vec{x}$ and field $\vec{E}$.

For free electrons equation of motion is

$$
m_{e} \frac{d^{2} \vec{x}}{d t^{2}}=e \vec{E}+F_{f}
$$

where $F_{f}$ is friction force that slows down the electron. The friction appears because of the collisions. Using collision frequency $v$, we can estimate this force as

$$
F_{f} \sim \delta p / \delta t \sim-m_{e} u / \tau \sim-m_{e} v \frac{d \vec{x}}{d t} .
$$

For laser field $\vec{E} \mathrm{e}^{-\mathrm{i} \omega t}$, solution of the equation (2.44) is 


$$
\vec{x}=-\frac{e / m_{e}}{i \omega v+\omega^{2}} \vec{E}
$$

Therefore conductivity $\sigma$ is

$$
\sigma=\frac{i n_{e} e^{2}}{m_{e}} \frac{\omega}{i \omega v+\omega^{2}}
$$

and dielectric function describing response of free electrons becomes

$$
\varepsilon(\omega)=1-\frac{4 \pi n_{e} e^{2}}{m_{e}} \frac{1}{i \omega v+\omega^{2}}=1-\frac{\omega_{p}^{2}}{\omega^{2}} \frac{1}{1+i v / \omega}=1-\frac{n_{e}}{n_{c r}} \frac{1}{1+i v / \omega}
$$

This formula is often called the Drude dielectric function. Two parameters were introduced in this expression - the plasma frequency $\omega_{p}^{2}=4 \pi m_{e} e^{2} / m_{e}$ and critical density $n_{c r}=m_{e} \omega^{2} / 4 \pi e^{2}$. In the extreme situation of a collisionless plasma, when electrons do not interact with each other $(v<<\omega)$

$$
\varepsilon(\omega)=1-\frac{\omega_{p}^{2}}{\omega^{2}}=1-\frac{n_{e}}{n_{c r}}
$$

Laser light can not propagate if its frequency is smaller than the plasma frequency or, equivalently, the electron density is larger than the critical density $n_{c r}=1.1 \cdot 10^{21} \mathrm{~cm}^{-3} / \lambda^{2}[, \mathrm{~m}]$. For one micron light and a cold NFE metal, the collision frequency is on the order of $10^{14} \mathrm{~Hz}$ (much smaller than $\omega-2 \cdot 10^{15} \mathrm{~Hz}$ ) and the electron density is on the order of $10^{23} \mathrm{~cm}^{-3}$ (much larger than the critical density $n_{c r} \sim 10^{21} \mathrm{~cm}^{-3}$ ). Thus, the dielectric constant is a large negative number on the order of -100 . The light can not propagate in such a medium and most of it will be reflected from the boundary. A 
small fraction of the energy is absorbed in a skin layer with thickness [Jackson98]

$$
l=\frac{\lambda}{4 \pi \operatorname{Im}[\sqrt{\varepsilon}]} .
$$

Using numbers presented above, we find a skin depth on the order of $10 \mathrm{~nm}$.

The laser energy deposition rate is proportional to imaginary part of dielectric constant [Jackson98]

$$
A=\frac{\omega}{8 \pi} \operatorname{Im}[\varepsilon] E^{2}=\frac{1}{2} \sigma E^{2}
$$

It is just Joule heating. Therefore, the absorption rate of free electrons is

$$
A=\frac{\omega_{p}^{2}}{8 \pi} \frac{v}{v^{2}+\omega^{2}} E^{2}=\frac{\omega_{p}^{2}}{8 \pi} \frac{\tau}{1+(\omega \tau)^{2}} E^{2} .
$$

In the previous section it was shown that in NFE metals, the collision frequency $v=1 / \tau$ first increases with temperature and than falls in the plasma region. According to (2.50) absorption of light from a typical USLP system should follow the same pattern: it is small at low temperatures, increases in the intermediary regime, and than diminishes for high temperatures. Since material temperature is proportional to the pulse energy, the dependence of absorption on laser fluence should follow the same behavior.

If the laser field interacts with bound electrons, the material response is different. Equation (2.44) should be expanded by the addition of a restoring force

$$
\frac{d^{2} \vec{x}}{d t^{2}}+\Omega^{2} \vec{x}+\Gamma \frac{d \vec{x}}{d t}=\frac{e}{m_{c}} \vec{E},
$$

where $\Omega$ is a material specific oscillation frequency and $\Gamma$ is a friction coefficient that 
includes effects of collisions. The conductivity and dielectric function become

$$
\begin{aligned}
& \sigma(\omega)=\frac{-i \omega \omega_{p}^{2}}{4 \pi\left(\Omega^{2}-\omega^{2}-i \omega \Gamma\right)} \\
& \varepsilon(\omega)=1+\frac{\omega_{p}^{2}}{\Omega^{2}-\omega^{2}-i \omega \Gamma}=1-\frac{\omega_{p}^{2}}{\omega^{2}-\Omega^{2}+i \omega \Gamma} .
\end{aligned}
$$

The optical properties are quite similar to the case of free electrons, except in one notable situation. When the laser frequency $\omega$ is close to resonance $(\omega \approx \Omega)$, the imaginary part of the dielectric constant is proportional to the inverse of $\Gamma$. This parameter includes the effects of oscillator friction $\Gamma_{0}$ and collisions: $\Gamma=\Gamma_{0}+v$. Therefore, light absorption goes down when the collision frequency increases. Since the material is modeled as a system of oscillators with friction, the above is effectively a response of a mechanical oscillator. Such a system absorbs energy at a slower rate when friction is larger [Landau76]. Response in the spectral domain has a bell-like curve with the peak at $\Omega$ and width $\Gamma$. Increase in $\Gamma$ broadens and lowers the peak.

Both of these models of dielectric function are widely used in a variety of situations. The Lorenz model is often applied in calculations of optical properties of gases and optical crystals [Siegman86]. The Drude model is successfully used for description of the dielectric function of ideal plasma [Ginzburg70] and metals interacting with far infrared light [Prokhorov90,Arnold84]. USLP absorption by NFE metals is a more complex case. Since the photon energy can be as high as several electron volts, we might see effects of interband transitions, when an electron is lifted from one energy band to 
another, for instance from valence to conduction band, via direct absorption of light quanta. It is suggested [Ehrenreich66] that effects of interband and intraband transitions can be split in two

$$
\varepsilon(\omega)=1+i \frac{4 \pi}{\omega}[\sigma(\text { intraband })+\sigma(\text { interband })]
$$

The effect of intraband transitions in NFE metal can be modeled with a modified Drude formula

$$
\sigma(\omega)=\frac{i n_{e} e^{2}}{M \omega m_{e}} \frac{1}{i \omega v+\omega^{2}}=\frac{i \omega_{p}^{2}}{M 4 \pi \omega} \frac{1}{i \omega v+\omega^{2}}
$$

where the constant $M$ describes the strength of intraband transitions. This can be interpreted as a modification necessary to account for the fact that the effective number of electrons $n_{e f f}$ participating in interband transitions is different from the true electron density $n_{e}$. Another explanation may be that electron's effective mass $m^{*}$ is different from $m_{e}$. In NFE metals the parameter $M$ typically has a value slightly larger than one.

On the other hand, calculation of interband contributions is much more complex and depends strongly on the band structure of the particular metal [Ashcroft71]. The complexity of the models, and, more often, their absence, forces us to look for a simple alternative. In the important case of parallel bands (bands that have the same energy derivative $\partial E / \partial p$ ) a modified version of the Lorentz model can be applied [Huttner94]

$$
\sigma(\omega)=-i \frac{\omega_{p}^{2} \omega}{4 \pi} \sum_{n} \frac{f_{n}}{\Omega_{n}^{2}-\left(\omega+i \Gamma_{n}\right)^{2}} .
$$


The summation is over different transitions with frequencies $\Omega_{n}$. Oscillator strengths $f_{n}$ describe their relative impact. Values of $\Omega_{n}$ are found from band structure calculation, the other parameters are fitted to give the best possible description of optical properties as a function of light frequency $\omega$. The model was applied to calculations of optical properties of aluminum [Huttner94] and lead [Huttner95].

Since typical USLP systems operate at a few specific wavelengths, we need to know behavior of $\varepsilon(\omega)$ only for a few frequencies, whereas temperature behavior is more interesting for us. We have used the following formula for the dielectric function

$$
\varepsilon=1-\frac{\omega_{p}^{2}}{M} \frac{1}{\omega(\omega+i v)}+\frac{A \omega_{p}^{2}}{\Omega^{2}-\left(\omega+i\left(\Gamma_{0}+v\right)\right)^{2}} .
$$

The interband contribution is represented by only one transition; however for cases when the transitions have either very similar or very different frequencies $\Omega$, it is a good approximation. For example in the case of aluminum, there are two parallel band transitions: at $3.4 \mu \mathrm{m}(0.35 \mathrm{eV})$ and at $0.82 \mu \mathrm{m}(1.5 \mathrm{eV})$ [Smith86]. At room temperature, the refractive index for specific wavelength can be found from a handbook [Palik98]. Therefore, we have two equations

$$
\left\{\begin{array}{c}
\varepsilon_{1}-1+\frac{\omega_{p}^{2}}{M} \frac{1}{\omega^{2}+v_{0}^{2}}=\omega_{p}^{2} \frac{A\left(\Omega^{2}-\omega^{2}+\left(\Gamma_{0}+v_{0}\right)^{2}\right)}{\left(\Omega^{2}-\omega^{2}+\left(\Gamma_{0}+v_{0}\right)^{2}\right)^{2}+4\left(\Gamma_{0}+v_{0}\right)^{2} \omega^{2}}=x_{1} \\
\varepsilon_{2}-\frac{\omega_{p}^{2}}{M} \frac{v_{0} / \omega}{\omega^{2}+v_{0}^{2}}=\omega_{p}^{2} \frac{2 A \omega\left(\Gamma_{0}+v_{0}\right)}{\left(\Omega^{2}-\omega^{2}+\left(\Gamma_{0}+v_{0}\right)^{2}\right)^{2}+4\left(\Gamma_{0}+v_{0}\right)^{2} \omega^{2}}=x_{2}
\end{array}\right.
$$

where $\varepsilon_{l}, \varepsilon_{2}$ are handbook values for real and imaginary parts of the dielectric function 
$\varepsilon(\omega), v_{0}$ is the collision frequency at room temperature. Parameter $M$ can be found elsewhere, for example, from behavior of electron heat capacity. That leaves us with three parameters - transition frequency $\Omega$, oscillator strength $A$ and transition width $\Gamma_{0}$. If we use $\Gamma_{0}$ as a fitting parameter, the rest can be found from the equations (2.56)

$$
\begin{aligned}
& \Omega^{2}=\omega^{2}-\left(\Gamma_{0}+v_{0}\right)^{2}+2\left(\Gamma_{0}+v_{0}\right) \omega \frac{x_{1}}{x_{2}} \\
& A=2 \frac{\left(\Gamma_{0}+v_{0}\right) \omega}{\omega_{p}^{2}} \frac{x_{1}^{2}+x_{2}^{2}}{x_{2}}
\end{aligned}
$$

To demonstrate how this Drude-Lorentz model works, we calculated absorption as a function of temperature for $800 \mathrm{~nm}$ and $400 \mathrm{~nm}$ light normally incident on an aluminum target. Aluminum is one of the few metals that have experimental data available for conditions other than room temperature. Figure 2.7 presents this absorption data together with room temperatures values for a range of wavelengths from $380 \mathrm{~nm}$ to $1000 \mathrm{~nm}$. Absorption of cold aluminum changes between five and thirteen percent. The peak occurs at $820 \mathrm{~nm}$, where parallel band transition takes place and as expected absorption curve has a bell-like shape. When the material is heated to $1550 \mathrm{~K}$ degrees, absorption increases moderately for practically all wavelengths except for those in the vicinity of $800 \mathrm{~nm}$, where absorption drops.

In the calculations, we have to account for the volume change of the heated material. At normal conditions $\left(\mathrm{P}=1 \mathrm{bar} \sim 10^{5} \mathrm{~Pa}\right.$ ) heated aluminum expands at the rate [Davis93] 


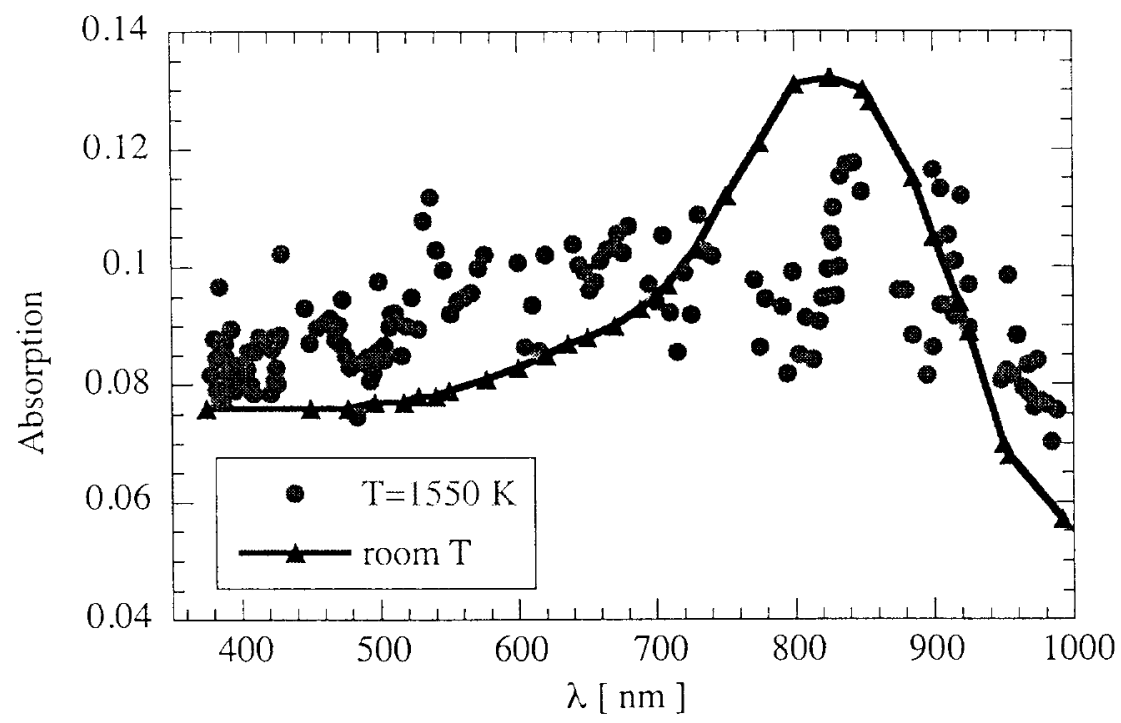

Figure 2.7: Experimental data of absorption versus wavelength for light incident normally on aluminum target at room temperature (blue line [Palik98]) and at $1550 \mathrm{~K}$ (red circles [Krishnan93a,Krishnan93b]).

$$
\begin{aligned}
& \beta_{\text {solid }}=\frac{1}{V} \frac{\partial V}{\partial T}=8.15 \cdot 10^{-5} \mathrm{~K}^{-1} \\
& \beta_{\text {liquid }}=\frac{1}{V} \frac{\partial V}{\partial T}=1.15 \cdot 10^{-4} \mathrm{~K}^{-1}
\end{aligned}
$$

Upon melting at $T_{m}=930 \mathrm{~K}$, the volume increases by 8 percent $\left(V_{S} / V_{L}=1.077\right)$. The change of volume is used to recalculate electron density. The collision frequency is dominated by electron-phonon encounters [Smith86,Davis93]

$$
\left\{\begin{array}{l}
v=9.1 \cdot 10^{13} \mathrm{~s}^{-1} \frac{T[\mathrm{~K}]}{300 \mathrm{~K}}, T<T_{m} . \\
v=1.54 v(T), T>T_{m}
\end{array} .\right.
$$

The parameter $M$ is equal to 1.6 [Smith86]. The dielectric function at room temperature was calculated from refractive index data: $n(800 \mathrm{~nm})=2.8+i 8.45$, 
$n(400 \mathrm{~nm})=0.49+i 4.86\left[\right.$ Palik98]. Parameter $\Gamma_{0}$ was set to $10^{14} \mathrm{~s}^{-1}$ for $800 \mathrm{~nm}$ light [Skobel'tsyn 73] and $2 \cdot 10^{15} \mathrm{~s}^{-1}$ for $400 \mathrm{~nm}$ wavelength. Our modeling showed that as long as the latter number is above $2 \cdot 10^{15} \mathrm{~s}^{-1}$ the results are practically unaffected.

In order to estimate the importance of interband contributions, the absorption was also calculated with the Drude model only (interband part of formula (2.55) was omitted). The Drude model is very frequently used to simulate optical properties of a metal with collision frequency adjusted to fit handbook value of absorption. Since collision frequency must be proportional to temperature, the match was achieved by multiplying the expression for $v$ with a numerical factor. This number is 15 for $800 \mathrm{~nm}$ and 8 for $400 \mathrm{~nm}$. As a result, for each wavelength we have three curves of absorption versus temperature: one is calculated using formula (2.55), another using the same formula without interband part and the third is Drude model fitted to room temperature absorption. Figure 2.8 and Figure 2.9 present results of our modeling, where absorption is given by the Fresnel formula for incidence from vacuum (or air) [Jackson98]

$$
A=1-\left|\frac{\sqrt{\varepsilon}-1}{\sqrt{\varepsilon}+1}\right|^{2} .
$$

Our calculations immediately yield one noticeable result - fitted Drude model produces a very significant growth of absorption with temperature. At $1550 \mathrm{~K}$ it gives approximately $50 \%$ absorption for both wavelengths - much larger than found in the experiment. The Drude model without the fitting of the collision frequency gives a 
similar relative increase, however the absolute magnitude is more reasonable and at high temperature we get $8-9 \%$ absorption, slightly below the experimental data. On the other hand, at room temperature it produces only $1 \%$ of absorption when it is supposed to be $13 \%$ for $800 \mathrm{~nm}$ and $7.5 \%$ for $400 \mathrm{~nm}$ light.

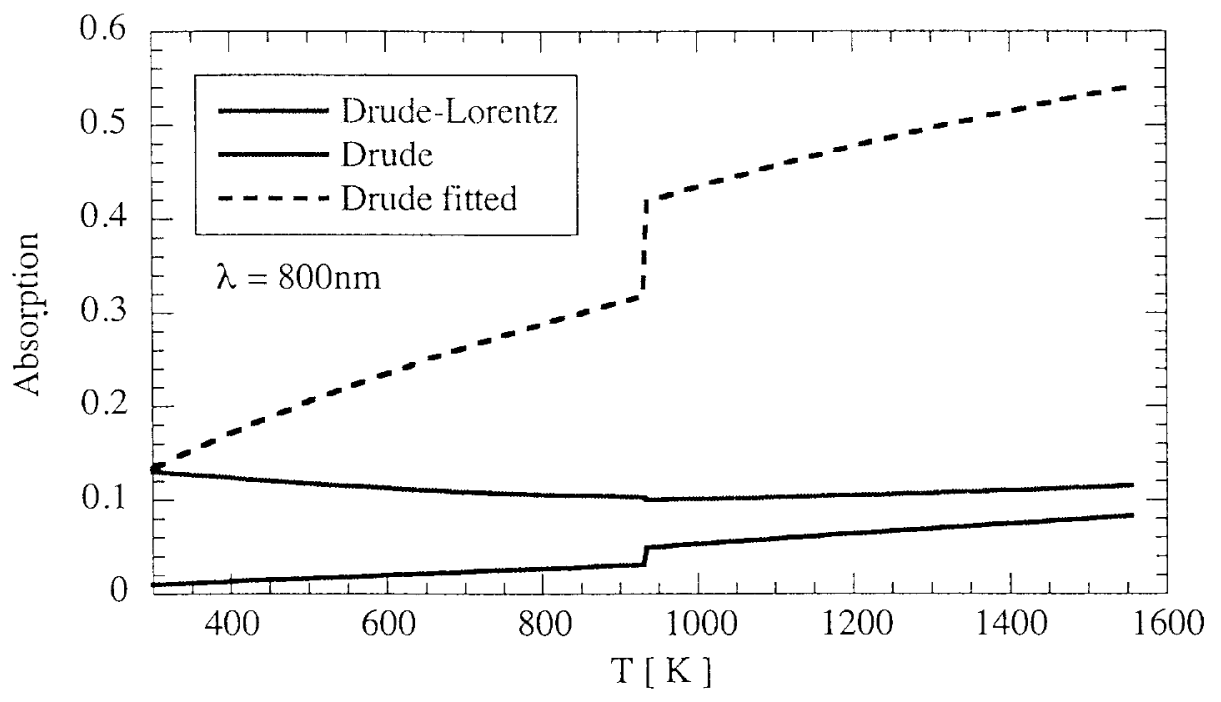

Figure 2.8: Absorption of $800 \mathrm{~nm}$ light incident normally on aluminum target as a function of temperature calculated with Fresnel formula. Solid red line is calculation with Drude-Lorentz model (Eq. (2.55)), solid blue line - only Drude model, dashed blue line Drude model with collision frequency fitted to give handbook absorption at room temperature.

The Drude-Lorentz model gives the most realistic answer. At room temperature, the result coincides with the experiment by definition. We observe absorption reduction with increase of temperature for $800 \mathrm{~nm}$ light and growth for $400 \mathrm{~nm}$ light. In both case the change is moderate as it is in the experiment. One interesting feature is that the impact of interband and intraband transitions changes in such a way that there is almost no change 
of absorption on melting for the $800 \mathrm{~nm}$ wavelength. If optical properties were described by the Drude model only, there would always be an increase of absorptivity on melting.

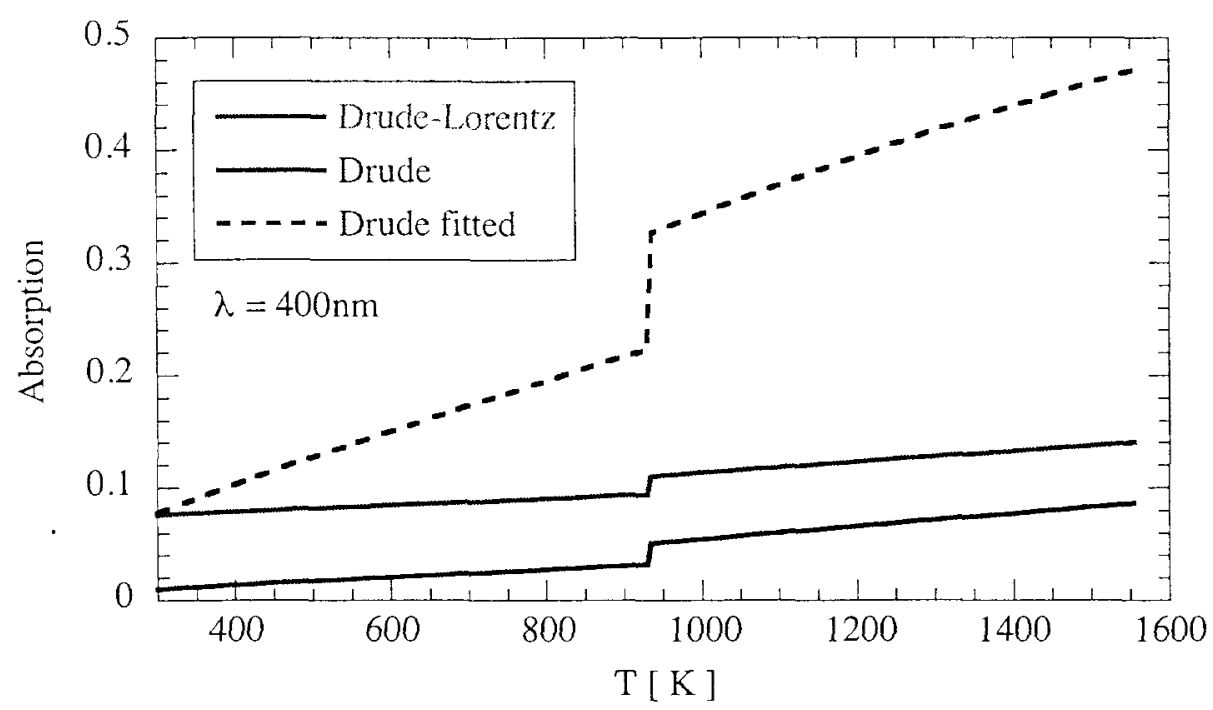

Figure 2.9: Absorption of $400 \mathrm{~nm}$ light incident normally on aluminum target as a function of temperature calculated with Fresnel formula. Solid red line is calculation with Drude-Lorentz model (Eq. (2.55)), solid blue line - only Drude model, dashed blue line Drude model with collision frequency fitted to give handbook absorption at room temperature.

With increased temperature, the contribution of interband transitions becomes less significant - an expected result, since thermal excitation of atoms destroys the symmetry on which band structure is based.

The Drude-Lorentz model does not always give a good quantitative agreement. In some cases, for example absorption of $400 \mathrm{~nm}$ light by copper, when effects of transitions from the d-band are important, the interband contribution is very large and it can not be described by the Lorentz model. Complex and extensive numerical calculations based on 
simulations of electron band structure [Stahrenberg01,Blaha00] are the only reliable approach. Unfortunately, the difficulty of this problem makes dynamic calculations of $\sigma($ interband) as a function of material state unfeasible. However, if one could do such a simulation, the answer would have been an interband conductivity $\sigma$ as a function of temperature and density. Such a function must tend to zero when kinetic energy of the atoms begins to dominate the potential energy of interaction and the band structure disappears. This happens when density significantly drops and/or temperature increases. The interband part in the Drude-Lorentz formula (2.55) certainly satisfies this requirement, thus it can be used as a first order approximation. This method certainly has an advantage over using an arbitrary temperature dependent formula, since it depends on collision frequency that can include different effects.

The Drude-Lorentz model gives the permittivity $\varepsilon$ as a function of material density and temperature for a wide range of parameters. Since we are interested in interaction with non-magnetic materials, this is all that is needed to solve Maxwell's equations and obtain reflectivity and absorption. This is, however, still potentially a very difficult task because of the disparity of characteristic scales. One needs to resolve the optical period both spatially and temporally. Thus the time step in the numerical calculations would have to be less than a femtosecond for a typical USLP. This constraint becomes even more severe when we take into account the fact that it is necessary to 
resolve the absorption skin depth, thus the size of the zone should be $1 \mathrm{~nm}$ or less. Due to the Courant type of numerical stability condition [Courant77] this requirement limits the numerical time step to values on the order of $10^{-17} \mathrm{~s}$. Since we need to model femtosecond and picosecond pulses, direct solution of the Maxwell's equations becomes unfeasible. One has to apply a method that is not so taxing numerically, but includes the effects of light reflection due to the sharp gradients of the dielectric function $\varepsilon$. Fortunately, because of the same disparity in parameters one can significantly simplify Maxwell's equations.

Assuming the vector fields are smooth enough, the original system of eight equations for electric and magnetic fields can be reduced to the wave equation for the electric field

$$
\Delta \vec{E}-\frac{1}{c^{2}} \frac{\partial^{2}(\varepsilon \vec{E})}{\partial t^{2}}=-\nabla\left(\frac{(\nabla \varepsilon, \vec{E})}{\varepsilon}\right) .
$$

Since the transverse dimensions of the laser deposition area are much smaller than the beam radius, one can assume that incoming and outcoming beams are plane waves (see Figure 2.10), that are represented by two linear polarizations: $\mathrm{TE}(\mathrm{S})$ and $\mathrm{TM}(\mathrm{P})$. A proper choice of the system of coordinates reduces equations (2.58) to the scalar wave equation for $\mathrm{S}$ polarization

$$
\Delta E_{x}-\frac{1}{c^{2}} \frac{\partial^{2}\left(\varepsilon E_{x}\right)}{\partial t^{2}}=0 .
$$


The right hand side term of the equation (2.58) is zero, because the gradient of the dielectric function $\varepsilon$ is orthogonal to the electric field in one dimensional planar case. $\mathrm{P}$ polarization is described by two equations for $E_{z}$ and $E_{y}$. Since we deal with plane waves, the field can depend on coordinates orthogonal to the $z$ axis only as

$$
f(z, x, y, t)=f(z, t) \exp \left(i k_{y} y\right)=f(z, t) \exp \left(i k_{0} \sin (\theta) y\right)
$$

where $k_{\theta}$ is the vacuum wave number of the laser and $\theta$ is angle of incidence (we consider incidence from vacuum). Substitution of this formula into

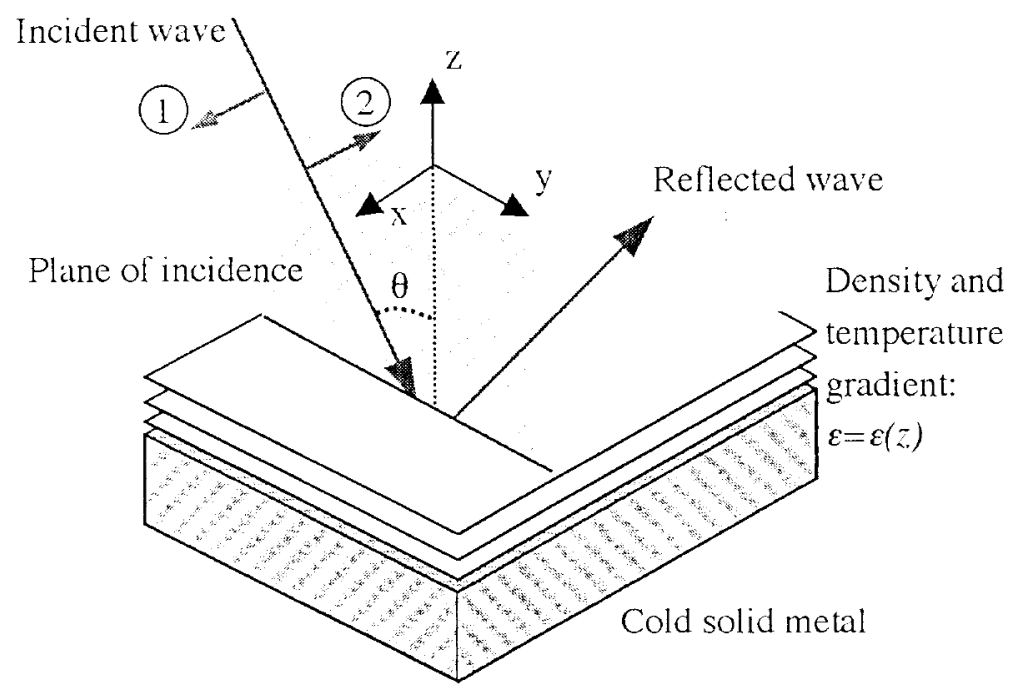

Figure 2.10: Schematic diagram of laser ablation. System of coordinates is aligned to have axis $z$ orthogonal to the material surface and $(z, y)$ plane to be parallel to the plane of incidence. Angle $\theta$ is angle between original direction of propagation of the incident wave and axis $z$ (beam path is curved when it propagates in the gradient of dielectric constant $\varepsilon$ ). Indices 1 and 2 designate TE(S) polarization - electric field is parallel to the surface plane, and TM(P) polarization - electric field lies in the plane of incidence.

the equation (2.59) reduces the number of parameters to two 


$$
\frac{\partial^{2} E}{\partial \tau^{2}}-k_{0}^{2} \sin (\theta)^{2} E-\frac{1}{c^{2}} \frac{\partial^{2}(\varepsilon E)}{\partial t^{2}}=0 .
$$

Optical fields can be split into a fast (carrier) and slow (envelope) time components

$$
f(z, t)=f^{\prime}(z, t) \exp (-i \omega t)
$$

The time derivative of the slow component describes effects of pulse propagation, however in our case thickness of the absorption zone is so small, that these effects can be neglected. Therefore one can omit derivatives of the slow component. Using dispersion relationship $c k_{0}=\varepsilon \omega$ we arrive to the Helmholtz equation for pulse envelope

$$
\frac{\partial^{2} E}{\partial z^{2}}+k_{0}^{2}\left[\varepsilon-\sin (\theta)^{2}\right] E=0
$$

If we use the same approximations, but begin with magnetic field, $\mathrm{P}$ polarized light also can be described with one equation for the slowly varying component of the magnetic field

$$
\frac{\partial^{2} B}{\partial z^{2}}-\frac{1}{\varepsilon} \frac{\partial \varepsilon}{\partial z} \frac{\partial B}{\partial z}+k_{0}^{2}\left[\varepsilon-\sin (\theta)^{2}\right] B=0
$$

Once the solution is known, the electric field can be found from the magnetic field using the relations

$$
E_{z}=\frac{\sin (\theta)}{\varepsilon} B, \quad E_{y}=\frac{i}{k_{0} \varepsilon} \frac{\partial B}{\partial z}
$$

and the laser power deposition density is calculated as

$$
S=k_{0} \frac{c \operatorname{Im}[\varepsilon]|E|^{2}}{8 \pi}
$$


The Helmholtz equation is an ordinary differential equation and it is much easier to solve than Maxwell's equations. This is how laser absorption is calculated in the hydrodynamics code described in the next section.

\subsection{HYADES - hydrodynamics code for self-consistent modeling of USLP}

\section{laser-material interaction}

Unequal temperature dynamics and light absorption are central parts of the simulation of the ultrashort laser material interaction. However the calculations can become truly versatile if one has an ability to describe changes in material density and ionization state that appear because of the laser action. Since these processes are mutually dependent, the modeling must be done in a self-consistent fashion, when the whole set of relevant equations is solved simultaneously. In this section we present an overview of the one-dimensional plasma hydrodynamics code HYADES [Larsen91,Larsen94,Larsen01] enhanced for USLP modeling. The code was made available by Jon Larsen, who kindly agreed to implement the models we developed for simulation of USLP interaction (light wave absorption and reflection, optical properties, low and moderate temperature collision frequency, thermal conductivity, electron-lattice energy exchange).

HYADES is a one-dimensional, three-geometry (planar, cylindrical, spherical), three-fluid radiation hydrodynamics code using the finite difference approach for numerical solution. The electron, ion and radiation components are treated as coupled and 
energy exchanging fluids in local thermodynamic equilibrium. Thermal transport is simulated in the flux-limited diffusion approximation; for radiation, the fluid multi-group diffusion prescription is employed. Thermodynamic quantities are derived from realistic equations of state. A number of models are available to describe the degree of ionization; however the material as a whole is neutral (there is no charge separation). The light absorption model includes wave effects and allows treatment of different laser polarizations and angles of incidence.

Hydrodynamic motion is a key component of the numerical modeling. All other physical processes such as thermal transport or laser energy deposition are connected to equations of motion as source terms. The equations of hydrodynamics are solved in the Lagrangian system of coordinates - the numerical mesh is "frozen" in the material and zone coordinates follow the flow of the material (see Figure 2.11). Such an approach is well suited for cases when significant changes of density are observed, for example shock waves propagation or material ablation.

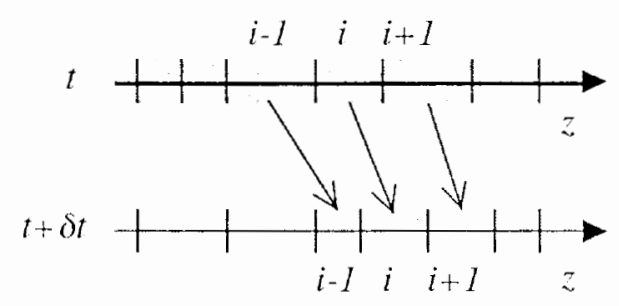

Figure 2.11: HYADES employs Lagrangian mesh. Pressure difference between zones causes the boundaries to move. Locations of mesh coordinates and zone density change with time in such a way that $z$ no masses are conserved. 
In the Lagrangian formulation, conservation of mass requirement is fulfilled automatically and one has to solve momentum and electron, ion and radiation energy conservation equations that in the planar configuration have the following form [Larsen01,Zel'dovich67]

$$
\begin{gathered}
\rho \frac{\partial u}{\partial t}=-\frac{\partial}{\partial z}\left(\sum_{j} P_{j}\right) \\
\rho \frac{\partial \varepsilon_{e}}{\partial t}+P_{e} \frac{\partial u}{\partial z}=-\frac{\partial q_{e}}{\partial z}+S_{L}-G_{e l}\left(T_{e}-T_{l}\right)+G_{e r}\left(T_{r}-T_{e}\right) \\
\rho \frac{\partial \varepsilon_{I}}{\partial t}+P_{I} \frac{\partial u}{\partial z}=-\frac{\partial q_{l}}{\partial z}+G_{e l}\left(T_{e}-T_{l}\right) \\
\frac{\partial U_{r}}{\partial t}+P_{r} \frac{\partial u}{\partial z}=-\frac{\partial q_{r}}{\partial z}+G_{e r}\left(T_{e}-T_{r}\right)+S_{e s}
\end{gathered}
$$

The parameters $u$ and $\rho$ are material velocity and density. $P, T, \varepsilon, U, q$ describe pressure, temperature, energy of electrons or ions, radiation energy and energy flux. Terms $G_{i j}\left(T_{i}-T_{j}\right)$ are responsible for energy exchange between the three "fluids". $S_{L}$ is energy deposition from the laser. $S_{e s}$ describes radiation energy that escaped from the material. HYADES can also treat a variety of other external sources and forces, however only terms relevant for our problem are listed. The last equation describes the radiation fluid, which is treated in the multi-group approximation. The radiation is divided into energy groups that are simulated separately, so that the total energy flux $q_{r}$ is a sum of fluxes over all photon groups. This method allows simple modeling of atomic structure and non Planckian statistics.

Pressure and internal energy as a function of density and temperature are obtained 
from a user specified equation of state which can be either in tabulated [Sesame83] or analytical form [Larsen01,More88,More91]. The degree of material ionization is calculated with the Thomas-Fermi model [Zel'dovich67,More91,Feynman49] - a semiclassical average-atom theory well suited for the description of metals. In order to have adequate modeling of pressure discontinuities (shock waves), the Von Neumann method of artificial viscous pressure is employed [Larsen01].

The collision frequency appears in a number of physical models. The calculations are based on the theory outlined in section 2.4. Total collision frequency $v$ is a sum of the electron-ion/phonon collision frequency $v_{\text {e-ph/l }}$ and the electron-electron collision frequency $v_{e-e}$. Interpolation between cold material and plasma regimes is done with the following formula

$$
v=\tau^{-1}=\left(\tau_{\text {cold }}^{2}+\tau_{\text {plusma }}^{2}\right)^{-1 / 2}
$$

The collision frequency is set to be no larger than the limiting frequency $v_{\text {lim }}=1 / \tau_{\text {lim }}$, calculated according to formula (2.42).

For calculation of the electron-ion collision time in plasma, the Spitzer formula modified to include effects of degeneracy [Lee84,More91,Larsen01] is used

$$
\tau_{e \prime}=\frac{3 \sqrt{m_{e}}\left(k_{B} T_{e}\right)^{3 / 2}}{2 \sqrt{2 \pi} n_{l} Z^{2} e^{4} \operatorname{Ln}(\Lambda)}\left(1+\exp \left(-\frac{\mu}{k_{B} T_{e}}\right)\right) F_{1 / 2}\left(\frac{\mu}{k_{B} T_{e}}\right),
$$

where $\mu$ is the chemical potential and $F$ is the Fermi-Dirac integral. The electron-electron collision time is $\tau_{e e}=Z \tau_{\iota^{\prime}}$. 
The following formula is employed for electron-phonon collision time [Ziman60]

in a relatively cold metal

$$
\tau_{e-p h h}=C_{0} C_{m e l t} \frac{R}{u_{F}} \frac{T_{m}}{T_{l}}
$$

where $R$ is interatomic distance, $u_{F}$ is the Fermi velocity, $T_{m}$ is the melting temperature and $T_{l}$ is the ion temperature. Constant $C_{m e l t}$ is used to describe the effect of melting. It equals one for a solid material and it becomes a number typically smaller than one in a liquid. Constant $C_{0}$ is used to fit experimental data. The melting temperature is a function of density and described by the Lindemann law [Steinberg91,Grover71,Royce71]

$$
T_{m}=T_{m 0} \exp \left(2 a\left(1-\rho_{0} / \rho\right)\right)\left(\frac{\rho}{\rho_{0}}\right)^{2\left(\gamma_{0}-a-1 / 3\right)},
$$

where $\rho_{0}$ is normal density and $T_{m 0}$ is the melting temperature at this density. Parameters $\gamma_{0}$ and $a$ describe Grüneisen coefficient $\gamma=\gamma_{0}+a\left(\rho_{0} / \rho-1\right)$ (formula (2.71) is discussed in more detail in the next chapter). When the density of a solid increases, the pressure becomes larger and it is more difficult to melt the material. The formula models the increase of melting temperature with density; for example, at atmospheric pressure aluminum begins to melt at temperature $930 \mathrm{~K}$ when density is $2.55 \mathrm{~g} / \mathrm{cm}^{3}$ [Davis93], however at normal density $\rho=\rho_{\nu}=2.7 \mathrm{~g} / \mathrm{cm}^{3}$ aluminum $\left(\gamma_{v}=1.97, a=1.5\right)$ melts at $1220 \mathrm{~K}$ [Seinberg91].

Electron-electron collisions in the solid are described by the modified formula (2.41) 


$$
\tau_{e e}=C_{e e} \frac{\hbar}{\varepsilon_{F}}\left(\frac{\varepsilon_{F}}{k_{B} T_{e}}\right)^{2},
$$

where $C_{e c}$ is a user specified constant, which is typically equal to one.

Figure 2.12 presents the collision frequency used in our calculations of optical properties of aluminum as a function of temperature at normal density. The electronphonon collision frequency at room temperature is $9.1 \cdot 10^{13} \mathrm{~s}^{-1}, C_{\text {melt }}=0.65$ and $C_{e e}=1$.

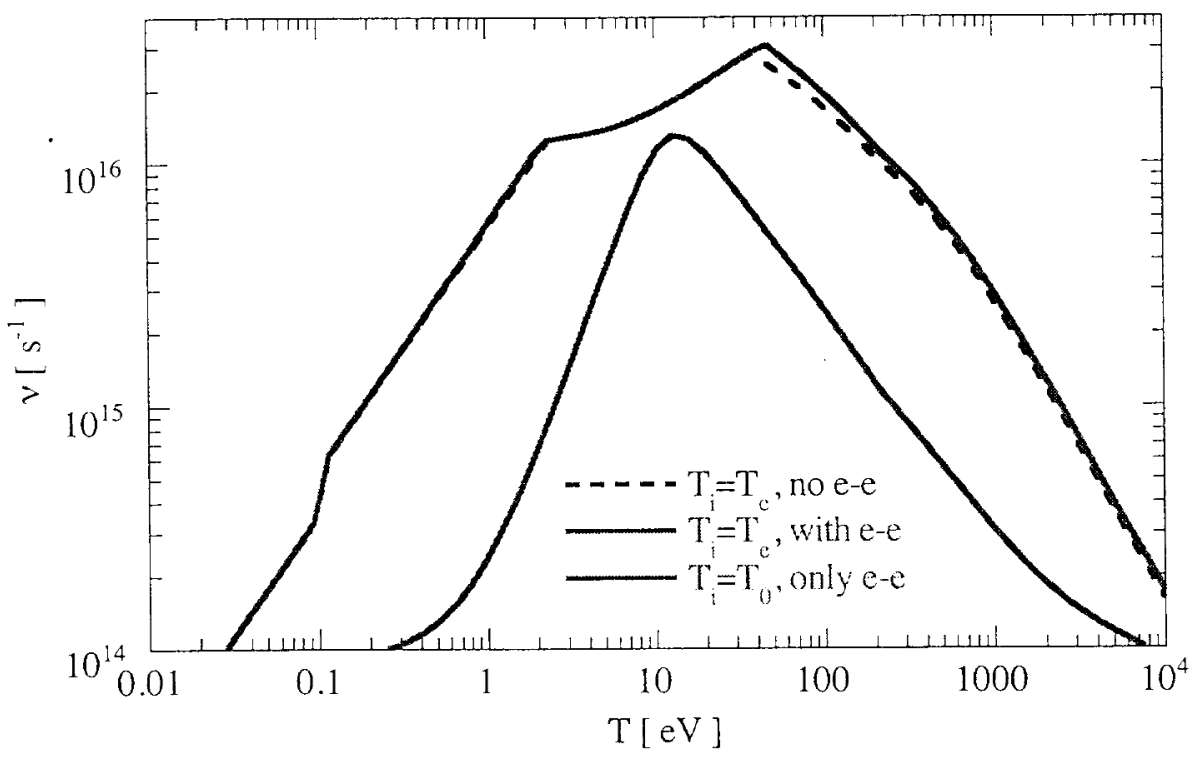

Figure 2.12: Collision frequency of aluminum at normal density $\rho=2.7 \mathrm{~g} / \mathrm{cm}^{3}$ calculated with HYADES. Red and blue curves are for equal ion and electron temperature without and with electron-electron collisions respectively. Green curve represents collisions when only electron temperature is varied and ion temperature is fixed at room temperature.

The plot shows that in the case of equilibrium, $\left(T_{i}=T_{e}\right)$, electron-ion/phonon collisions are dominant (the reason why electron-electron collisions rarely attract attention). If the 
lattice is cold and only electron temperature is varied, increase in collision frequency becomes noticeable when electron temperature approaches $1 \mathrm{eV}$.

According to the flux-limited diffusion model, energy fluxes $q_{i}$ in the equations (2.67) are calculated as

$$
q=-\max \left(\chi \frac{\partial T}{\partial z}, q_{m}(\rho, T)\right)
$$

where $q_{m}$ is maximum energy flow. This model is used because for very sharp temperature gradients the diffusion approximation breaks down [Key91] and gives unphysically high energy flux. The maximum energy flux $q_{m}$ is typically set to a small fraction $(\sim 0.05-0.1)$ of free streaming energy flow, which for example for electrons is $n_{e} k_{B} T_{e}\left(k_{B} T_{e} / m_{e}\right)^{1 / 2}$

The thermal conductivity of the electrons is calculated in a fashion similar to the collision frequency - interpolation between cold metal and plasma models. At low temperatures, thermal conductivity is given by

$$
\chi_{e}=\frac{\pi^{3}}{3} \frac{n_{e} k_{B}\left(k_{B} T_{e}\right)}{m_{e} v}
$$

and in the plasma regime [More91,Lee84] it is given by

$$
\chi_{e}=\frac{n_{e} k_{B}\left(k_{B} T_{e}\right)}{m_{e} v} A^{\beta}\left(\frac{\mu}{k_{B} T_{c}}\right) .
$$

Here $v$ is the total collision frequency and $A^{\beta}$ is a slowly varying function. For ideal plasma $\left(\mu / k_{B} T_{e} \rightarrow \infty\right)$ it is equal to $128 / 3 \pi$, the collision frequency is descried by the 
formula (2.38) and the Spitzer expression [Spitzer62] for thermal conductivity is recovered

$$
\chi_{e}=8\left(\frac{2}{\pi}\right)^{3 / 2} \frac{k_{B}\left(k_{B} T_{e}\right)^{5 / 2}}{\sqrt{m_{e}} e^{4} Z \ln (\Lambda)}
$$

In a solid, when temperature is low and electrons and ions are in equilibrium, the collision frequency is inversely proportional to temperature, thus heat conductivity coefficient is approximately constant. Constant $C_{0}$ in the formula (2.70) for electronphonon collision frequency is used to match experimental data at room temperature.

Figure 2.13 shows a comparison of the calculated conductivity with the experimental data for aluminum for temperature up to $1000 \mathrm{~K}$.

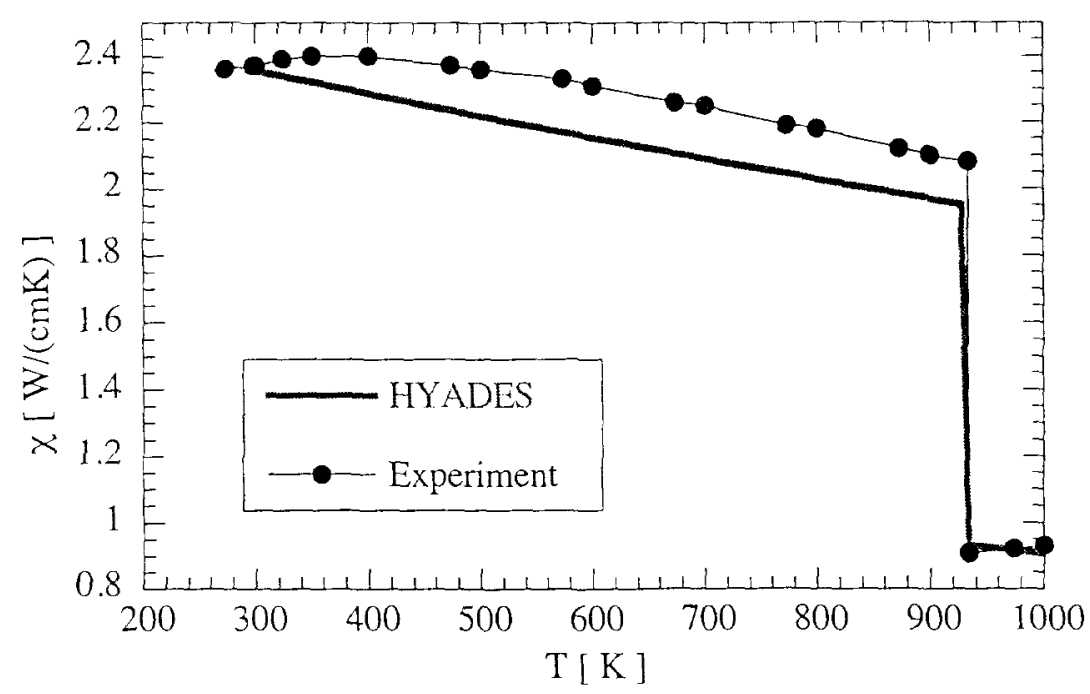

Figure 2.13: Thermal conductivity of aluminum as a function of temperature at normal conditions (atmospheric pressure). Red curve is calculated with HYADES and black points are experimental data [Davis93]. 
It shows a slight decrease in thermal conductivity of a solid with temperature, which is caused by the change in material volume due to thermal expansion. Agreement of the theoretical numbers with experiment is within ten percent.

Thermal transport of ions is handled by an expression similar to (2.76) and it gives a conductivity much smaller than the corresponding number for electrons. Energy transport by the radiation fluid is much more complex [Larsen01]. However, in the next section it will be shown that, at least for moderate laser pulse energies, it is not important.

The energy exchange coefficient $G_{e I}$ is taken as

$$
G_{e l}=\frac{3 n_{l} k_{B}}{2 \tau_{e q}}
$$

In the plasma regime, the equilibration time is calculated as [Larsen01,Brysk74]

$$
\tau_{e q}=\frac{3 \pi m_{l} \hbar^{3}}{8 m_{e}^{2} e^{4} Z^{2} \ln (\Lambda)}\left[1+\exp \left(-\frac{\mu}{k_{B} T_{e}}\right)\right]=\frac{m_{I}}{2 m_{e} Z} \tau_{e l} .
$$

As expected, the equilibration time is $m_{l} / m_{e}$ times larger than the collision time. At low temperatures this formula gives a smaller equilibration time than the one observed in experiments. In order to compensate for this deviation, the equilibration time for low temperature metal is calculated as

$$
\tau_{e q, c} \sim \frac{m_{l}}{2 m_{e}} \tau_{e-p h}=C_{0} \frac{m_{l}}{2 m_{e}} \frac{R}{u_{F}} \frac{T_{m}}{T_{I}}
$$

The constant $C_{\theta}$ in this case is chosen in such a way that $G_{e l}$ matches experimental data at room temperature. Interpolation between these two regimes is done according to formula 
(2.68). Similarly to collision frequency calculations, the equilibration time is set to be no smaller than $\tau_{\text {lin }} m_{l} / 2 m_{e}$.

The optical properties of the material are simulated with the slightly modified Drude-Lorentz model described in the previous section. Material at low density or very high temperature has periodic symmetry broken and band effects should disappear. In order to insure this change, the actual values of parameters $M$ and $A$ describing the strength of intraband and interband transitions are set to

$$
\begin{aligned}
& M=1+\left(M_{0}-1\right) f\left(\rho, T_{I}\right), \\
& A=A_{0} f\left(\rho, T_{I}\right)
\end{aligned}
$$

where subscript 0 denotes parameters calculated from user input of dielectric constant at room temperature for laser wavelength and parameter $\Gamma_{0}$. The function $f(\rho, T)$ must equal unity at normal conditions and decrease to zero for high temperature and low density. We chose it to be

$$
\begin{aligned}
& f\left(\rho, T_{I}\right)=1, T_{I}<T_{\text {melt }}(\rho) \\
& f\left(\rho, T_{l}\right)=\exp \left(-\left(T_{l}-T_{m e l t}\right) / T_{\text {melt }}\right), T_{I}<T_{\text {melt }}(\rho)
\end{aligned}
$$

where $T_{\text {melt }}$ is calculated according to (2.71). This choice is somewhat arbitrary; however it is natural to expect the melting temperature to be a characteristic temperature of this process. Fortunately, our simulations show that for modeling of USLP interaction the exact functional dependence of $f(\rho, T)$ is not significant.

In order to calculate light reflection and absorption, HYADES solves equation 
(2.63) for $\mathrm{S}$ polarized light and equation (2.64) for $\mathrm{P}$ polarized light. Since we use a stratified medium (optical properties are only a function of depth) approximation, a multilayer representation of the material and corresponding matrix formalism [Born89, Milchberg89] is employed. The formalism of equations (2.63) and (2.64) allows polarization and angle of incidence to be taken into account within the one dimensional stratified medium description used in the code. The dielectric function $\varepsilon$ in each layer is considered to be constant and the solution of the Helmholtz equations is trivial - two exponentials in each layer. At the boundaries of the layers, the two neighboring solutions can be matched with an equivalent of the Fresnel formulas. Both of these relationships are linear equations connecting amplitudes of the waves. If we represent the amplitudes of incoming and outcoming waves in a layer $i$ as $A_{i}$ and $B_{i}$, they can be related to the same values in the previous, nearer to the surface, layer through matrix multiplication

$$
\left(\begin{array}{l}
A_{i} \\
B_{i}
\end{array}\right)=P_{i} T_{i-1, i}\left(\begin{array}{l}
A_{i-1} \\
B_{i-1}
\end{array}\right)=P_{i} T_{i-1, i} \cdots P_{1} T_{0,1}\left(\begin{array}{l}
A_{0} \\
B_{0}
\end{array}\right)=M_{i}\left(\begin{array}{l}
A_{0} \\
B_{0}
\end{array}\right)=\left(\begin{array}{ll}
m_{i, 11} & m_{i, 12} \\
m_{i, 21} & m_{i, 22}
\end{array}\right)\left(\begin{array}{l}
A_{0} \\
B_{0}
\end{array}\right)
$$

where $P_{i}$ represents the propagation matrix for layer $i, T_{i-1, i}$ is transmission matrix from layer $i-l$ to $i, M_{i}$ is total matrix for $i$ layers and $A_{0}, B_{0}$ are amplitudes of the original incident and reflected waves. The matrices $P$ and $T$ are functions of the layer dielectric constant and layer thickness. Initial amplitude $A_{0}$ is calculated from user specified laser intensity. In order to calculate reflected amplitude $B_{0}$ we have to propagate the wave to a layer $j$ where its amplitude is very small and reflected wave $B_{i}$ is zero. Thus from 
equation (2.82) we have

$$
0=m_{i, 21} A_{0}+m_{i, 22} B_{0}
$$

and reflected amplitude can be found. Once the fields are known, absorption is calculated according to formula (2.66). If layers are sufficiently thin, this method gives a good approximation of the solution.

In order to verify that our complex code produces reasonable results, the calculations were benchmarked against three available, different experimental sets of data for USLP absorption in a wide range of pulse intensities. Such experiments are very difficult to perform - one must take great care to ensure laser pulse and target quality. All of the measurements are for aluminum. The experiments were performed in a vacuum with vapor-deposited films. Scattering was reported to be insignificant in all experiments. HYADES simulations were done using the SESAME tabulated equation of state for aluminum \#3718 [Sesame83]. Necessary optical parameters were specified in the previous section. The electron-ion energy exchange constant at room temperature is set to $2 \cdot 10^{11} \mathrm{~W} /\left(\mathrm{cm}^{3} \mathrm{~K}\right)[$ Eidmann00].

Milchberg et. al. [Milchberg88] presented the first experimental study of ultrashort laser pulse absorptivity. They measured $308 \mathrm{~nm}$ light reflection for a $400 \mathrm{fs}$ Gaussian pulse at $45^{\circ}$ angle of incidence for both $\mathrm{S}$ and $\mathrm{P}$ polarizations. Figure 2.14 compares experimental and theoretical absorption versus peak pulse intensity in the range from $10^{11} \mathrm{~W} / \mathrm{cm}^{2}$ to $10^{15} \mathrm{~W} / \mathrm{cm}^{2}$. The theoretical data was adjusted to account for the 
Gaussian laser spot. The experimental and numerically calculated curves exhibit the same behavior. At low intensities, absorption is small, then increases with pulse energy, peaks at a certain intensity and then begins to drop.

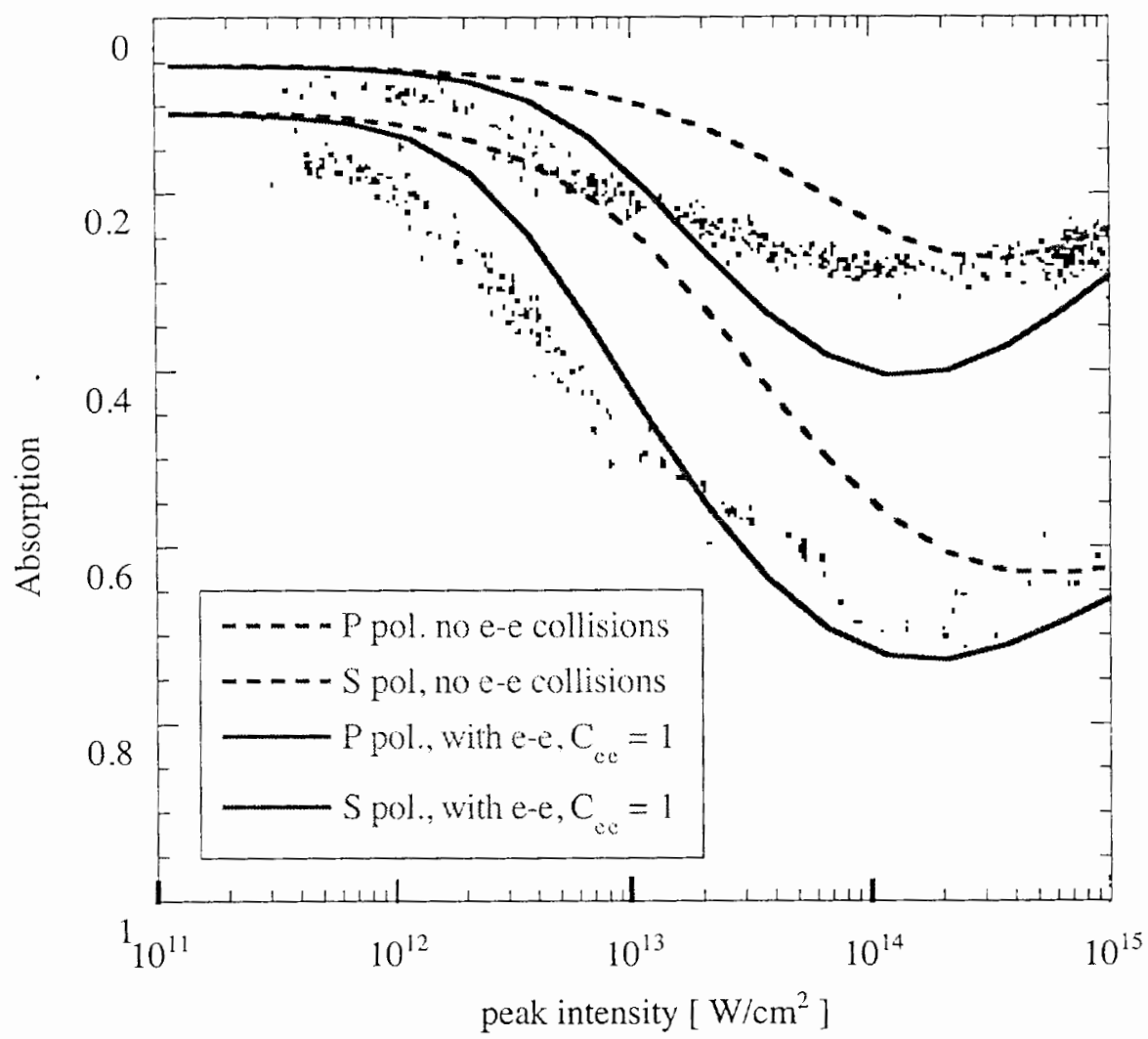

Figure 2.14: Experimental [Milchberg88] and HYADES theoretical results for absorption versus peak pulse intensity for $308 \mathrm{~nm}, 400 \mathrm{fs}$ laser pulse incident on aluminum at $45^{\circ}$. Theoretical calculations were done for two cases: with and without electronelectron collisions. Black dots represent experimental data.

Qualitatively, this picture is valid for both polarizations, however the P polarized pulse has noticeably higher absorption. Theoretical calculations that include the effect of 
electron-electron collisions give the best match to the data. Absorption peaks are located at the right intensity: $1 \div 2 \cdot 10^{14} \mathrm{~W} / \mathrm{cm}^{2}$. For P polarization, the value of the absorption peak is very close to the experiment - approximately 70 percent. However, for S polarization it is higher than it should be according to the experiment ( $40 \%$ vs. $30 \%$ ). At low intensities, HYADES gives absorption that is somewhat lower than in the experiment. It is hard to tell what produces this difference, since our model of optical properties at low temperatures reproduces absorption calculated from handbook data for dielectric constant [Palik98].

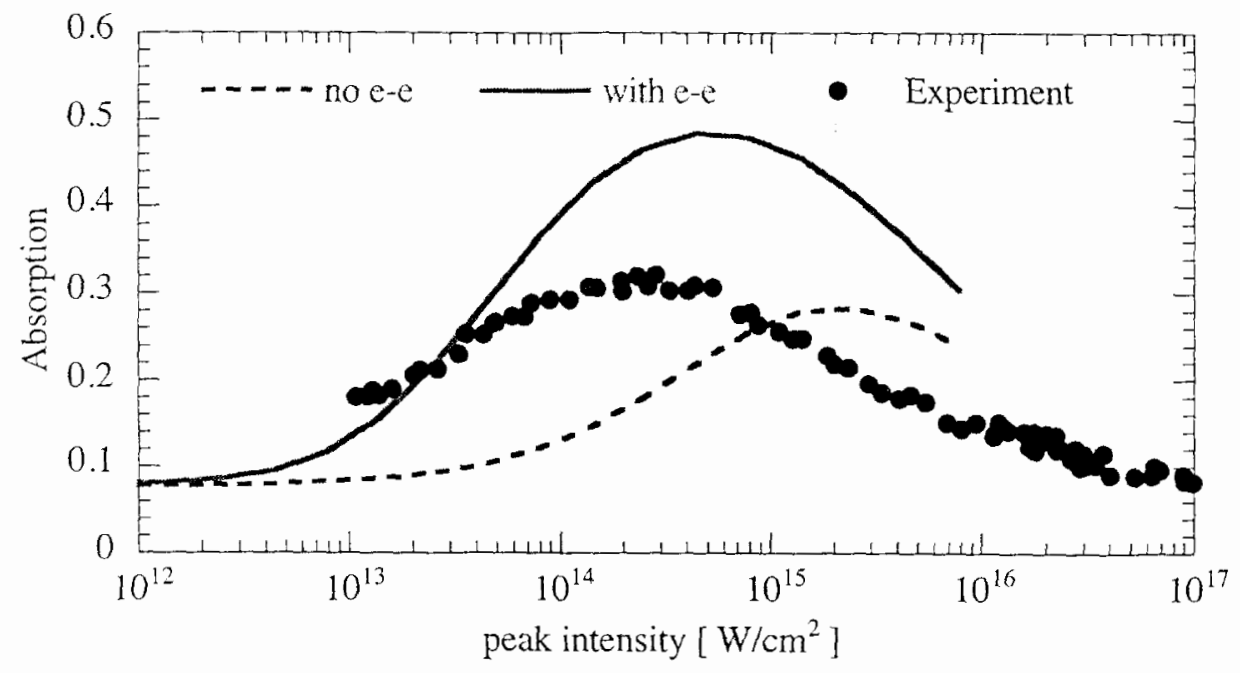

Figure 2.15: Experimental [Price95] and HYADES theoretical results for absorption versus peak pulse intensity for $400 \mathrm{~nm}, 150 \mathrm{fs}$ laser pulse incident normally on aluminum. Theoretical calculations were done for two cases: with and without electron-electron collisions. Black dots represent experimental data.

The next set of experimental data is from Price et.al. [Price95]. They measured absorption of $150 \mathrm{fs}$ pulses normally incident on aluminum for a range of intensities from 
$10^{13} \mathrm{~W} / \mathrm{cm}^{2}$ to $10^{17} \mathrm{~W} / \mathrm{cm}^{2}$. The pulse is $400 \mathrm{~nm}$ light produced by frequency doubling the output of a Ti:sapphire laser. Figure 2.15 shows that the qualitative behavior of absorption is similar to the previous example, however quantitative agreement between experiment and theory is not as good. The peak of absorption appears in our calculations at a somewhat higher intensity $\left(4 \div 6 \cdot 10^{14} \mathrm{~W} / \mathrm{cm}^{2}\right.$ vs. $\left.2 \div 3 \cdot 10^{14} \mathrm{~W} / \mathrm{cm}^{2}\right)$ and the absorption value is noticeably higher (50\% vs. $30 \%$ ) than found in the experiment.

The most recent experimental data for USLP absorption by aluminum is from Fisher et.al. [Fisher01]. Using a Ti:Sapphire laser, they measured absorption of a $50 \mathrm{fs}$ normally incident beam both for $800 \mathrm{~nm}$ and $400 \mathrm{~nm}$ light. Figure 2.16 compares

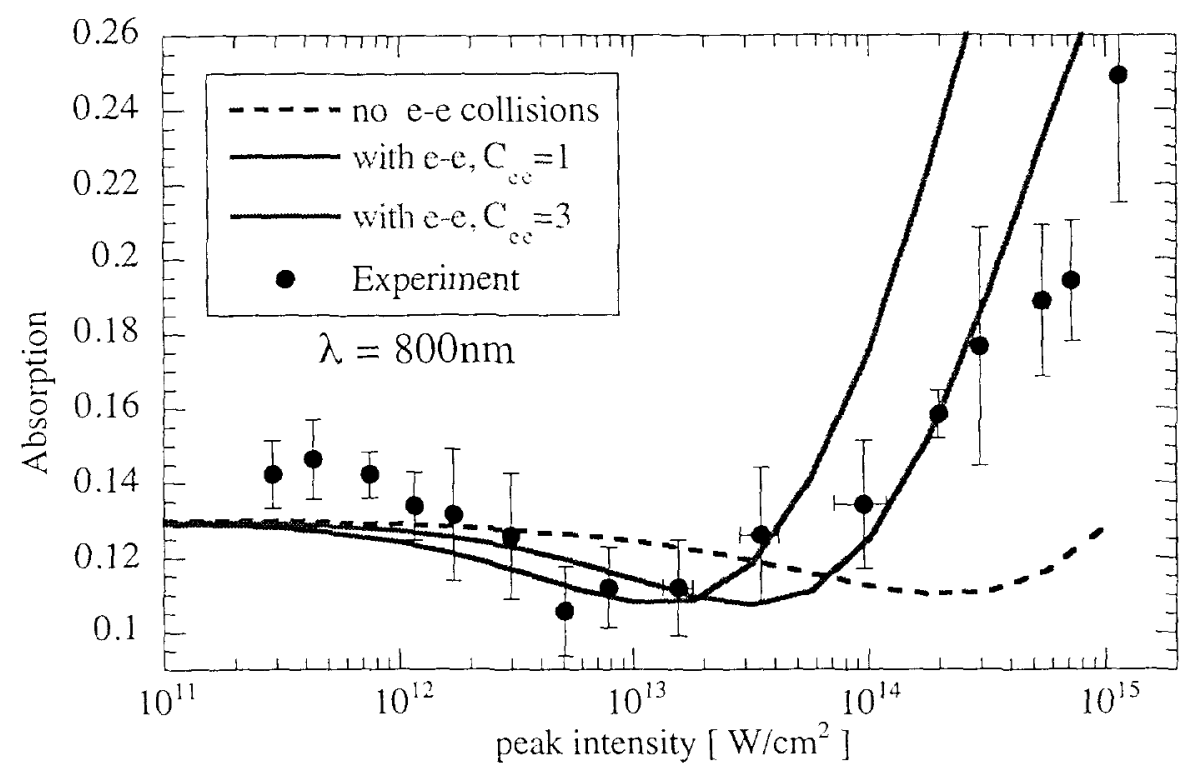

Figure 2.16: Experimental [Fisher01] and HYADES theoretical results for absorption versus peak pulse intensity for $800 \mathrm{~nm}, 50 \mathrm{fs}$ laser pulse incident normally on aluminum. 
experimental results and HYADES calculations for $800 \mathrm{~nm}$ light. At low intensity $\sim 10^{11} \mathrm{~W} / \mathrm{cm}^{2}$, experimental absorption is $14 \%$ - very close to $13 \%$. This is the absorption of cold aluminum according to reference data [Palik98] (result is reproduced by HYADES). Absorption begins to decrease slowly for higher intensities due to the interband effects. It reaches a minimum of $\sim 11 \%$ at $10^{13} \mathrm{~W} / \mathrm{cm}^{2}$ (approximately $0.5 \mathrm{~J} / \mathrm{cm}^{2}$ laser fluence). Once this minimum is reached, absorption slowly goes up and at $10^{14} \mathrm{~W} / \mathrm{cm}^{2}$ reaches the absorptivity of cold aluminum. Above this intensity absorption begins to increase much faster, reaching approximately $25 \%$ at $10^{15} \mathrm{~W} / \mathrm{cm}^{2}$ intensity. HYADES calculations have the same qualitative behavior, however the plot clearly demonstrates that one must include electron-electron collisions in order to have a good quantitative match. If e-e collisions are neglected the absorption rises noticeably slower. Since formula (2.72) for electron-electron collisions is more like an estimate, we tried to adjust the $C_{c e}$ parameter to get the best description of the experiment. If this parameter is left at its default value of one, HYADES gives an excellent match up to several $\mathrm{J} / \mathrm{cm}^{2}$ pulse fluences. For higher laser energies better agreement is achieved with $C_{e t}=3$. A larger coefficient $C_{e e}$ means the electron-electron collision frequency is smaller than the one predicted by the original formula (2.41) [Abrikosov72]. Since the formula is based on the assumption that $T_{e}<<T_{F}$, it very well might be overestimating the collision frequency for the case when considerable pulse energies significantly heat the electrons. 


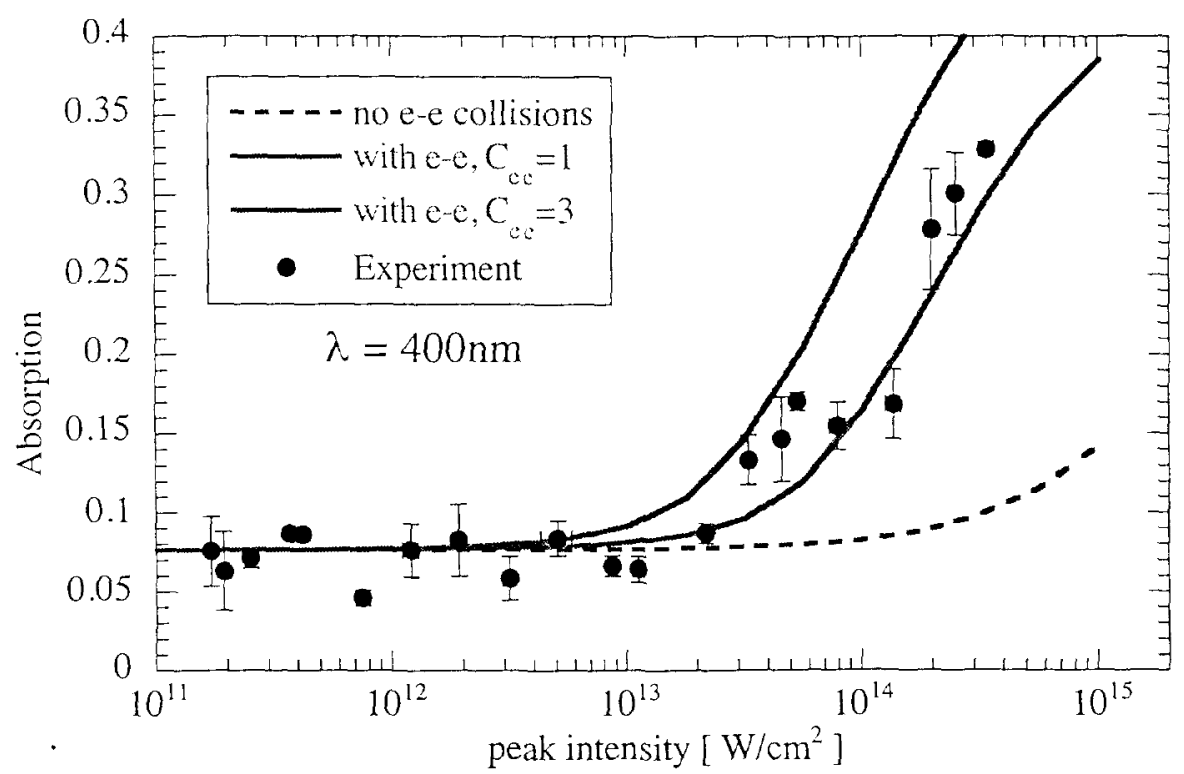

Figure 2.17: Experimental [Fisher01] and HYADES theoretical results for absorption versus peak pulse intensity for $400 \mathrm{~nm}, 50 \mathrm{fs}$ laser pulse incident normally on aluminum.

Figure 2.17 demonstrates results for $400 \mathrm{~nm}$ light. In this case interband transitions have a smaller contribution and the absorption stays flat at approximately $7.5 \%$ until laser fluence reaches a few $\mathrm{J} / \mathrm{cm}^{2}$. After that it begins to grow, however at a much sharper rate than in case of $800 \mathrm{~nm}$ light. Again HYADES gives the best match to experimental data if electron-electron collisions are included.

The main conclusion from our benchmarking is that HYADES can reproduce qualitative and, in many cases, quantitative behavior of USLP absorption for a variety of laser parameters. It tends to overpredict absorption at high intensities, where nonideal plasma plays an important role. Unfortunately, simple theories for description of this regime do not exist. However, for moderate pulse energies, HYADES simulations 
produce good results, demonstrating the importance of e-e collisions and interband transitions.

\subsection{USLP absorption}

In this section USLP absorption by aluminum (an example of NFE metal) as a function of laser parameters is described in more detail. Electron-electron collisions are always included, and the parameter $C_{e e}$ is set to one.

In section 2.3 , temperature dynamics was studied for rectangular pulses in a very simple approximation. We repeat this study, but with HYADES. First, let's review absorption of low energy USLP. Figure 2.18 shows the evolution of peak temperature during absorption of a Gaussian, $800 \mathrm{~nm}, 100 \mathrm{fs}, 10^{12} \mathrm{~W} / \mathrm{cm}^{2}$ laser pulse - similar to the case presented in Figure 2.4. Widths of electron temperature and laser energy deposition profiles are also plotted. The pulse fluence is approximately $0.1 \mathrm{~J} / \mathrm{cm}^{2}$ and $11.2 \%$ of this energy is absorbed, which is slightly lower than the $13 \%$ room temperature absorption. Material heating is not strong enough to affect optical properties significantly. Another indication of this is that during the interaction the width of the laser energy deposition zone is practically a constant $6 \mathrm{~nm}$ (this number is slightly smaller than the $7.7 \mathrm{~nm}$ skin depth of $800 \mathrm{~nm}$ light because it is defined as the full width half maximum, instead of the l/e width criterion for skin depth). The width of the electron temperature profile is determined initially by the absorption zone and is equal to $12 \mathrm{~nm}$. 


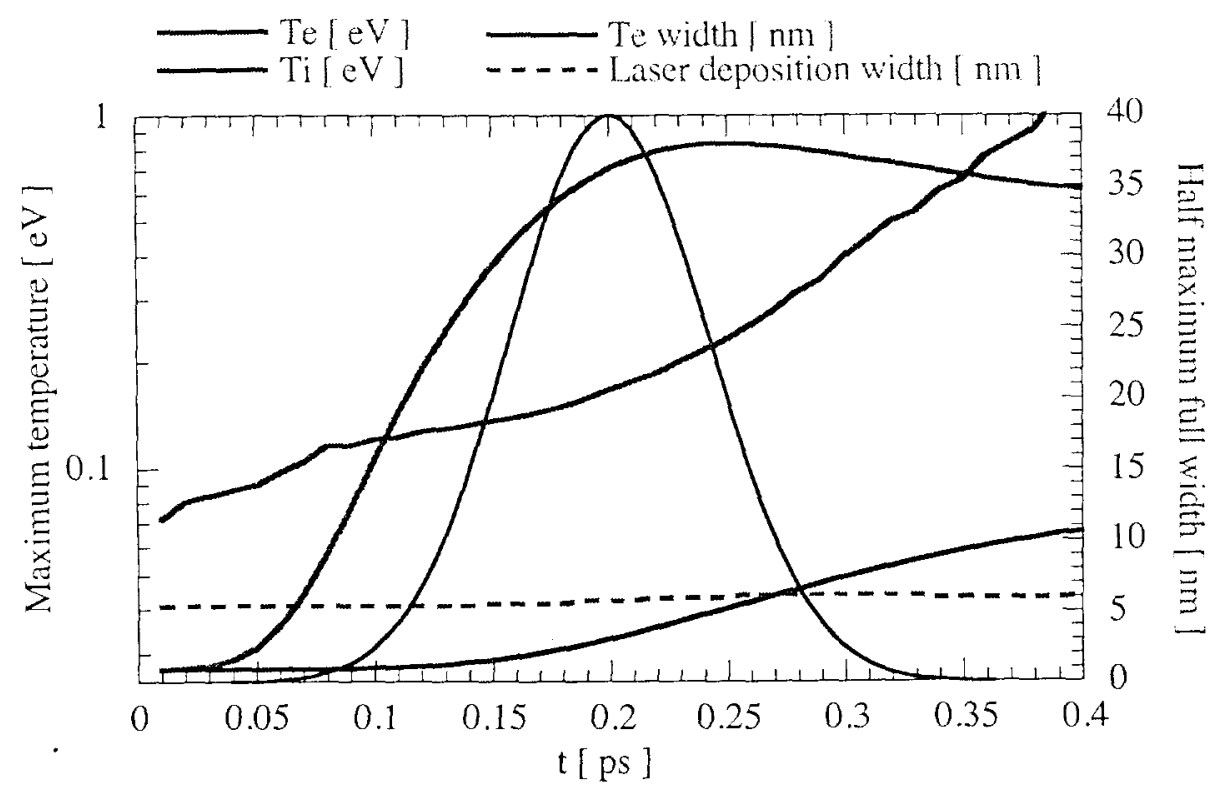

Figure 2.18: Evolution of maximum electron and ion temperature in aluminum driven by the normally incident $800 \mathrm{~nm} 10^{12} \mathrm{~W} / \mathrm{cm}^{2}$ Gaussian pulse (shown with a black line). Change in width of electron temperature and laser deposition profiles is shown with green curves.

Since the electron energy is proportional to $T_{e}^{2}$ and energy deposition density $S_{L}$ has an exponential profile, it is twice as big as the width of $S_{L}$. By the time the peak of the pulse arrives, the temperature profile expands to $20 \mathrm{~nm}$ due to thermal diffusion. After intensity begins to drop, the spreading accelerates and by the time the pulse is over it reaches $42 \mathrm{~nm}$. Maximum temperatures achieved during the pulse are similar to our estimates in section 2.3: $0.83 \mathrm{eV}(9600 \mathrm{~K})$ for electrons and $0.067 \mathrm{eV}(780 \mathrm{~K})$ for ions. Even at the end of the pulse, the lattice temperature is below the melting temperature.

More energetic laser pulses increase material temperatures high enough to affect 
optical properties. Figure 2.19 shows profiles of electron and ion temperatures together with laser power deposition density $S_{\llcorner}$at the peak of the normally incident laser pulses with $100 \mathrm{fs}$ length and $5 \mathrm{~J} / \mathrm{cm}^{2}$ fluence (peak intensity is $4.7 \cdot 10^{13} \mathrm{~W} / \mathrm{cm}^{2}$; for Gaussian pulses the following relationship is valid: $F=1.06 I_{\text {peak }} \tau$, where $\tau$ is conventionally defined at full width half maximum). Two cases are presented: $800 \mathrm{~nm}$ and $400 \mathrm{~nm}$ light interaction. One can immediately see distorted profiles of energy deposition density.

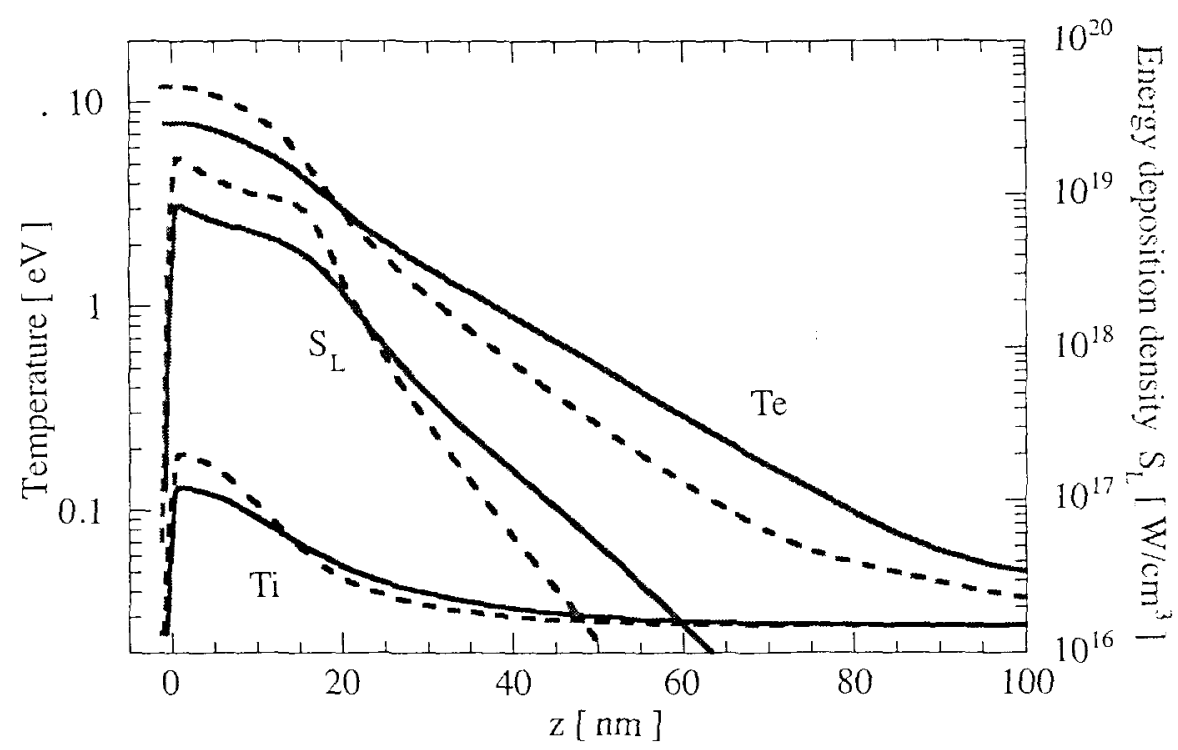

Figure 2.19: Plot of electron (red) and ion (blue) temperatures together with laser energy deposition $S_{L}$ (green) versus distance at the peak of the pulse with fluence $5 \mathrm{~J} / \mathrm{cm}^{2}$, length lo0fs and normal incidence (results for $800 \mathrm{~nm}$ light - solid curves, for $400 \mathrm{~nm}$ - dashed curves). Aluminum occupies region of positive $z$. The laser is incident from the left.

In the first $20 \mathrm{~nm}$ it decays noticeably slower than in the case of colder material - the half width is approximately $15 \mathrm{~nm}$ for both wavelengths. In the deeper region it returns to the usual exponential behavior determined by the $7.7 \mathrm{~nm}$ and $6.5 \mathrm{~nm}$ skin depths for $800 \mathrm{~nm}$ 
and $400 \mathrm{~nm}$ light respectively. Electron temperature reaches as high as $10 \mathrm{eV}$ and ion temperature exceeds the melting temperature. The width of the electron temperature profile is approximately $15 \mathrm{~nm}$ - close to the size of the deposition zone. For temperatures this high, the electron gas begins to behave more like a regular gas with internal energy proportional to temperature. The picture shows that energy deposition from the $400 \mathrm{~nm}$ laser pulse is higher, as a result total absorption is $41.5 \%$ for the $400 \mathrm{~nm}$ pulse and $25 \%$ for the $800 \mathrm{~nm}$ pulse.

Despite the fact that ion temperature is relatively low, significant electron pressure initiates material expansion before the laser pulse is over. In a cold metal material pressure is dominated by the response of ions, which is approximately linear in temperature. The electron thermal contribution is much smaller due to the Fermi distribution effects and behaves as a temperature squared

$$
P_{e T}=-\partial U_{e} / \partial V \sim T_{e}^{2}
$$

At low laser fluences, the pressure produced by the electrons is still small and noticeable expansion can begin only after temperature equilibration. However, for the case presented in Figure 2.19, the electron temperature is comparable to the Fermi temperature and the electron contribution is predominant (see Figure 2.20). The electron gas begins to expand, but electrostatic forces are much stronger. The electrons can not separate from ions, thus pulling them out and starting the material expansion. 


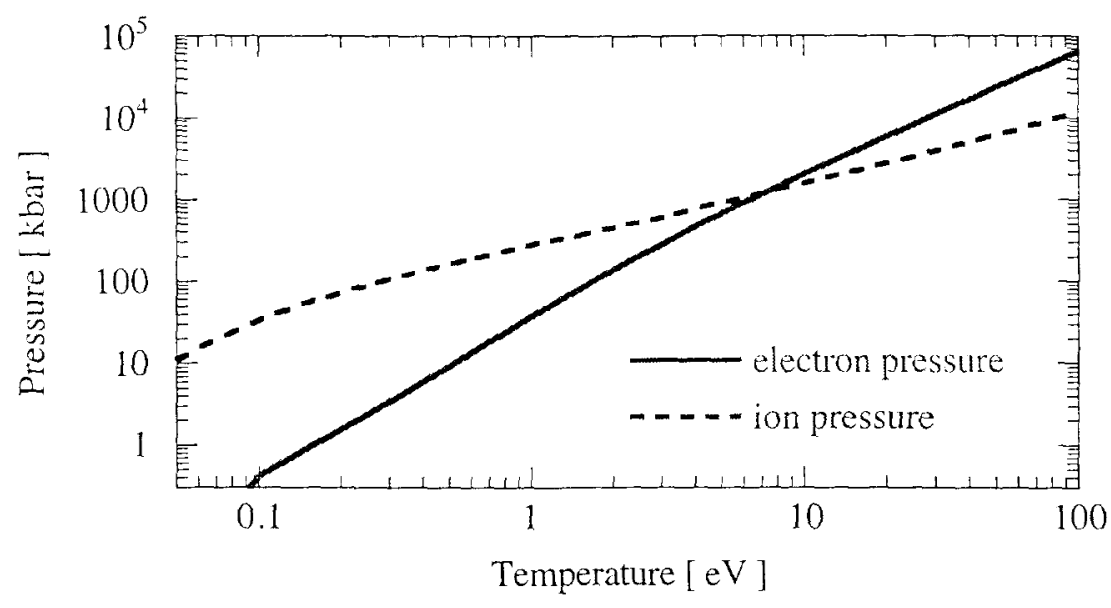

Figure 2.20: Plot of electron and ion pressure versus temperature for aluminum at normal density $\rho=2.7 \mathrm{~g} / \mathrm{cm}^{3}$. Data is from SESAME EOS \#3718 [Sesame83] used in the calculations.

Figure 2.21 presents $(z, t)$ color plots that describe change in important material parameters for some time after the pulse is over. Evolution of electron and ion temperatures, pressure and density for the $800 \mathrm{~nm}$ pulse is tracked up to $3.8 \mathrm{ps}$ after the pulse peak. The highest electron temperature of $11.5 \mathrm{ev}$ is reached close to the material surface about 80 fs after the pulse peak. Such high temperature creates pressures in the Mbar range, which initiate material expansion with a velocity approximately $50 \mathrm{~km} / \mathrm{s}$. By $200 \mathrm{fs}$ after the pulse peak, an ejecta plume $10 \mathrm{~nm}$ thick is already created. Simultaneously, the pressure wave begins to propagate in the bulk of the material with supersonic speed of approximately $10 \mathrm{~km} / \mathrm{s}$ (sound speed in aluminum is $6.4 \mathrm{~km} / \mathrm{s}$ ). Since the energy deposition zone is so thin, a rarefaction wave almost immediately 

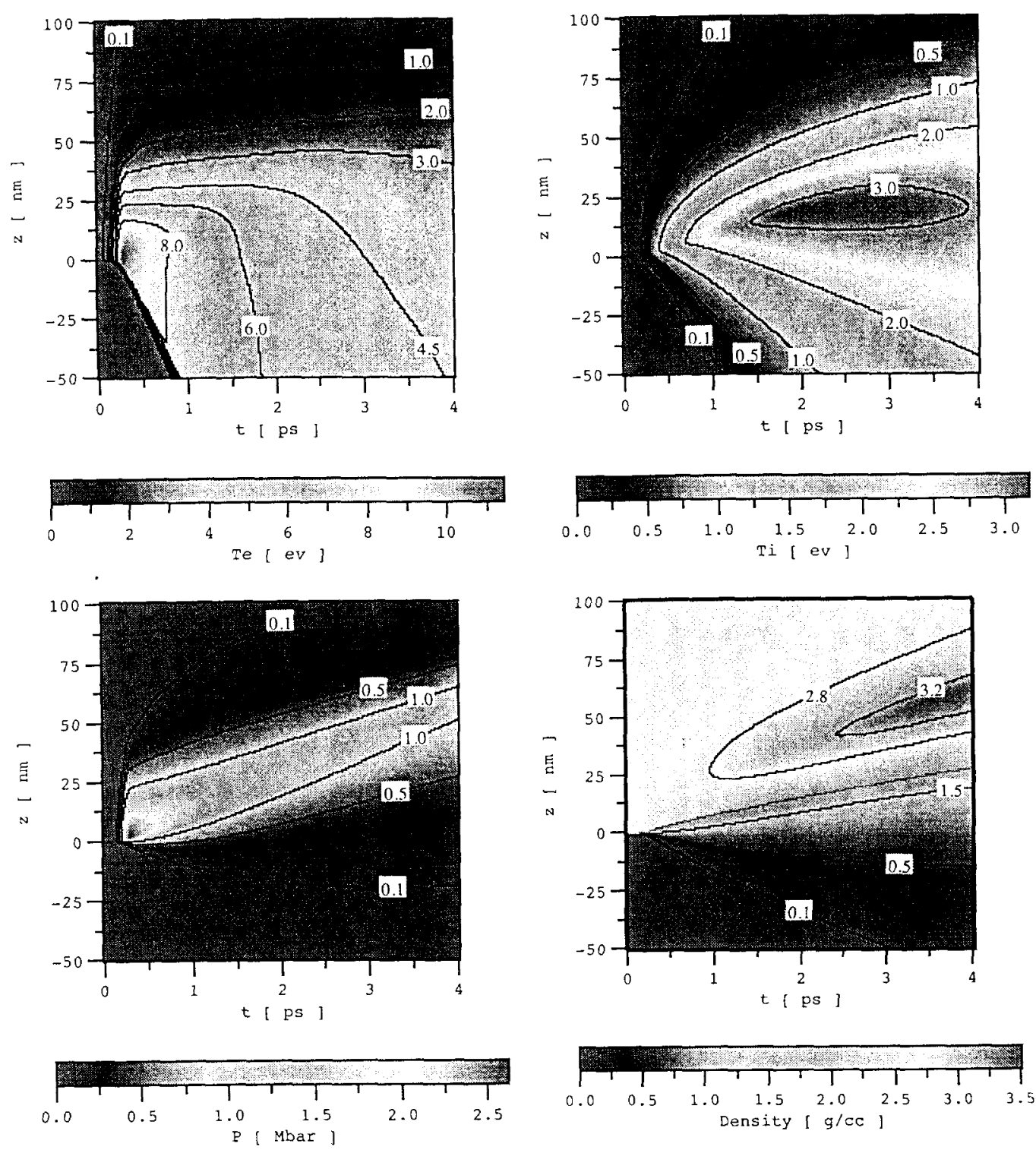

Figure 2.21: $(z, t)$ color plots of evolution of electron and ion temperatures, pressure and density of aluminum after absorption of normally incident laser pulse with fluence $5 \mathrm{~J} / \mathrm{cm}^{2}$, width $100 \mathrm{fs}$ and wavelength $800 \mathrm{~nm}$. The beam was directed along the $z$ axis (from bottom to top) and the pulse peak is at $0.2 \mathrm{ps}$. At $t=0$ aluminum occupies region of positive $z$. Red line on the density plot shows a zero material velocity contour. 
begins to interact with the compression wave and the pressure drops from $2.6 \mathrm{Mbar}$ at its peak to $1.1 \mathrm{Mbar}$ after $3.8 \mathrm{ps}$. As it moves into the bulk, high compression of aluminum is reached, however a true shock (discontinuous jump in pressure and density) is not formed yet. At the same time, material behind the compression front continues to be ejected. The red line on the density plot shows the zero material velocity contour - at the end of the run aluminum layer with original thickness $22.5 \mathrm{~nm}$ is turned into a hot, expanding plume and the ablation process is still underway. At the same time electron and ion temperatures in the denser part of the material have almost come into an equilibrium with the highest temperature of approximately $3 \mathrm{eV}$. Electron temperature is still slightly larger, however in this regime ions contain most of the thermal energy. Heated material extends to a significant depth: at the end of the calculation, the layer of material with temperature above $0.08 \mathrm{eV}$ (melting temperature of aluminum) is $155 \mathrm{~nm}$ thick.

In order to understand how material parameters change in the interaction with laser beams with different energies, a set of calculations was performed for a range of pulse fluences. Figure 2.22 shows light absorption as a function of laser fluence for $400 \mathrm{~nm}$ and $800 \mathrm{~nm}$ pulses with $100 \mathrm{fs}$ width together with calculations for $800 \mathrm{~nm}$, $1 \mathrm{ps}$ pulses. The results are similar to the behavior we observed when HYADES was benchmarked against experimental data. At a low fluence of $0.01 \mathrm{~J} / \mathrm{cm}^{2}\left(I \sim 10^{11} \mathrm{~W} / \mathrm{cm}^{2}\right)$ light absorption is determined by cold material properties; it is $0.13 \%$ for $800 \mathrm{~nm}$ and 


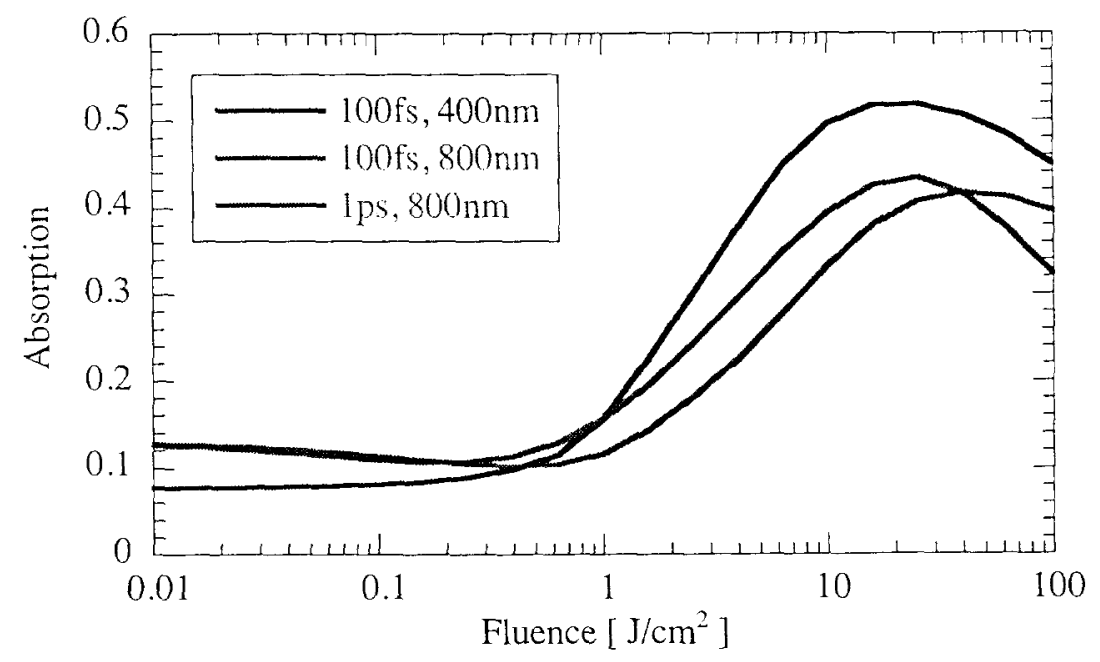

Figure 2.22: Absorption of aluminum as a function of fluence of normally incident laser pulse. The curves are for $400 \mathrm{~nm}$ wavelength, $100 \mathrm{fs}$ pulse width (blue), $800 \mathrm{~nm}, 100 \mathrm{fs}$ width (red) and $800 \mathrm{~nm}, 1 \mathrm{ps}$ width (green).

$7.5 \%$ for $400 \mathrm{~nm}$ [Palik98]. With increase of pulse energy, the absorption begins to decrease for $800 \mathrm{~nm}$ and increase for $400 \mathrm{~nm}$ light and around $0.4 \mathrm{~J} / \mathrm{cm}^{2}\left(I \sim 3.7 \cdot 10^{12} \mathrm{~W} / \mathrm{cm}^{2}\right)$ it becomes approximately $10 \%$ for all three cases. Further increase of laser fluence initiates a sharp growth in the absorption that continues until fluence reaches several tens of $\mathrm{J} / \mathrm{cm}^{2}$. At this point it comes to a peak $(\sim 0.5$ for $400 \mathrm{~nm}$ light, $\sim 0.4$ for $400 \mathrm{~nm}$ light $)$ and then begins to decrease. The temperature becomes so high that the metal is turned into a plasma, where collision frequency decreases with temperature. For USLP with width of lps, absorption evolution is similar to that of $100 \mathrm{fs}$ pulses, but shifted to lower fluences. At moderate intensities it happens because there is more time for energy exchange between electrons and ions and at high laser energies effects of material expansion 
become important.

Figure 2.23 presents the calculated maximum pressure and temperatures at the end of the pulse for these three cases. In order to discount the effects of absorption, the parameters are plotted versus absorbed fluence.
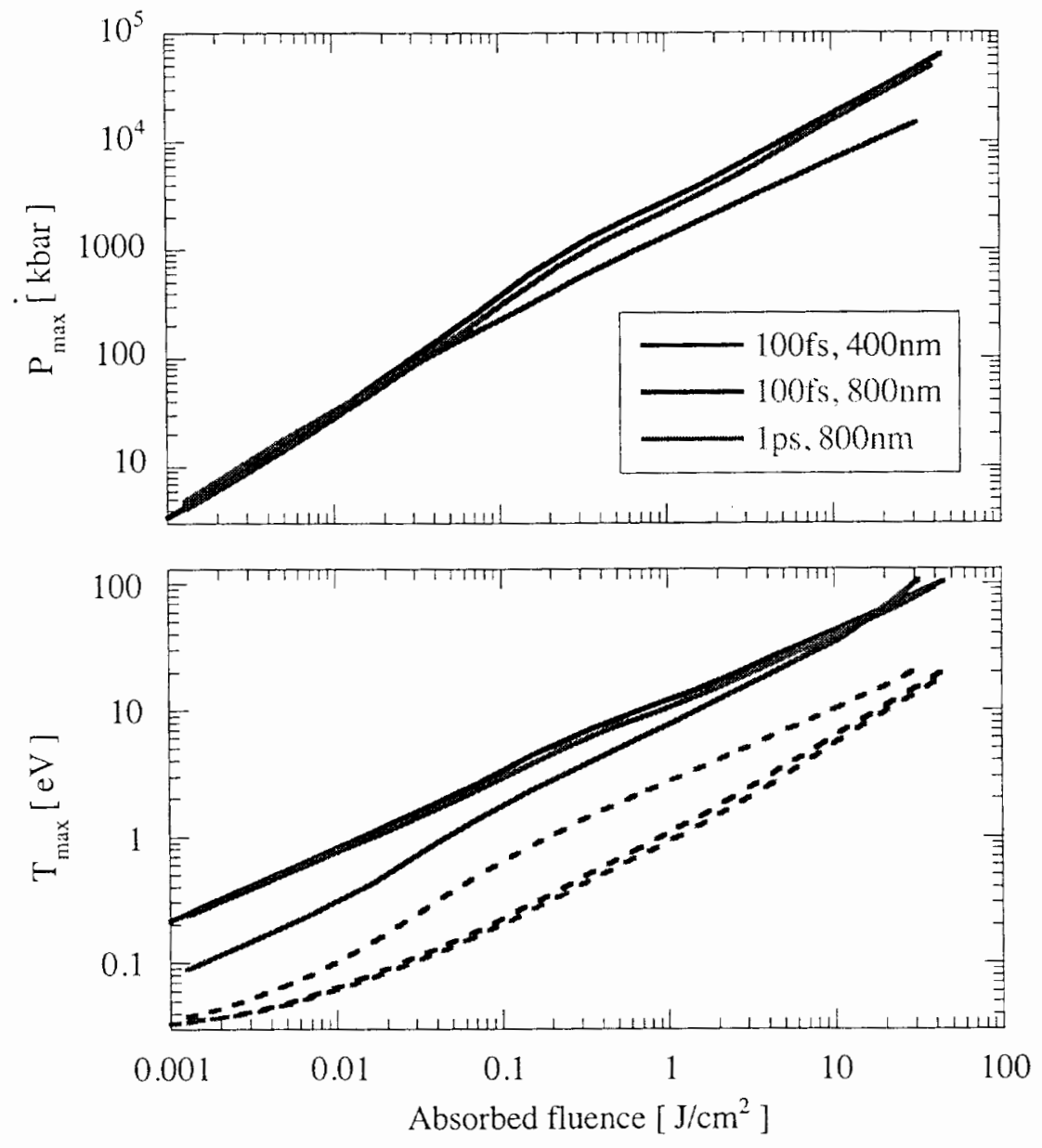

Figure 2.23: Plot of maximum pressure (top) and electron and ion temperature (bottom) at the end of the pulse ( $2 \tau$ after the pulse peak) versus absorbed fluence for the three cases presented on Figure 2.22. On the temperature plot solid lines correspond to electron temperature and dashed curves are ion temperature 
The curves for $400 \mathrm{~nm}$ and $800 \mathrm{~nm} 100 \mathrm{fs}$ pulses are practically the same. For an ultrashort puise, the only effect a change of light wavelength has is to change the amount of absorbed energy. A sharp increase in absorption begins when the pulse fluence is approximately $0.4-0.6 \mathrm{~J} / \mathrm{cm}^{2}$. This corresponds to absorbed fluence $0.04-0.06 \mathrm{~J} / \mathrm{cm}^{2}$. The graph shows that at this point electron temperature begins to exceed $1 \mathrm{eV}$. As we learnt in the previous section, this is when electron-electron collisions start to make a significant contribution to the total collision frequency. We can also notice that, approximately in the same range of fluences, ion temperature exceeds the melting temperature - another boost to absorption. The most energetic laser pulse in our calculation is $100 \mathrm{~J} / \mathrm{cm}^{2}$. This corresponds to a light intensity of almost $10^{15} \mathrm{~W} / \mathrm{cm}^{2}$ and generates peak electron temperature of $100 \mathrm{eV}$. This in turn creates pressure of almost $100 \mathrm{Mbar}$. Such pressure initiates expansion with velocities above $100 \mathrm{~km} / \mathrm{s}$. Since the laser photon carries a momentum $h k$, the light also exerts pressure when a photon is absorbed or reflected. It is equal to $(2-A) I / c$, where $A$ is absorption, $I$ is intensity and $c$ is light speed. For the highest intensity case, this corresponds to approximately $1 \mathrm{Mbar}$, which is well below the pressure appearing due to energy absorption. Thus it can be neglected.

Light interaction with metal for a longer pulse length of 1 ps results in a lower peak electron and higher ion temperature. Figure 2.21 demonstrates that in a solid material it can happen in a few picoseconds, however peak electron and ion temperatures are located in different places. Electron temperature peaks on the front of the expanding 
plume. This is a region that has low density due to the expansion. When density drops, the electron-ion energy exchange time increases and the lower ion temperature is frozen in the front of the plume. However, in the deeper part that is not yet affected by the expansion, the equilibration does happen quickly. The rarefaction wave propagating with a speed $\sim 10^{6} \mathrm{~cm} / \mathrm{s}$ reaches depth $\sim 20 \mathrm{~nm}$ in a couple of picoseconds. The ion temperature peaks approximately at this depth.

In the previous section it was mentioned that radiation has a small effect on the process ${ }^{*}$ of absorption. This was verified by tracking efficiency of conversion from laser energy to radiation energy. The calculations show that it is very low. For the highest intensity pulse $\left(I=10^{15} \mathrm{~W} / \mathrm{cm}^{2}, \tau=100 \mathrm{fs}\right)$, the efficiency was $2.5 \cdot 10^{-4}$. Most of this energy escapes the material. Conversion efficiency does grow with temperature, however experiments show that even for very energetic and intense pulses $\left(I=10^{18} \mathrm{~W} / \mathrm{cm}^{2}\right.$, $\tau=300 \mathrm{fs}$ ), it is only a few percent [Jiang95]. Therefore radiation doesn't have a significant role in thermal transport or hydrodynamic behavior in the interaction of USLP with a metal. Unless there is interest in the radiation itself, radiation transport can be omitted.

Previous simulations almost always were done for normal incidence. In order to study effects of the angle of incidence and polarization we calculated the absorption as a function of incidence angle for $800 \mathrm{~nm}$ light pulses for two different pulse lengths and intensities. Results are shown on Figure 2.24 together with absorption of solid, cold aluminum. The qualitative behavior of absorption for different laser parameters is the 
same as in the case of cold metal, when it is given by the Fresnel formulas (incidence from vacuum or air) [Jackson98]

$$
\begin{gathered}
A(P)=1-\left|\frac{\sqrt{n^{2}-\sin (\theta)^{2}}-n^{2} \cos (\theta)}{\sqrt{n^{2}-\sin (\theta)^{2}}+n^{2} \cos (\theta)}\right|^{2} \\
A(S)=1-\left|\frac{\sqrt{n^{2}-\sin (\theta)^{2}}-\cos (\theta)}{\sqrt{n^{2}-\sin (\theta)^{2}}+\cos (\theta)}\right|^{2}
\end{gathered}
$$

At the normal incidence absorption is the same for both polarization (as it should be, since $P$ and $S$ polarized waves become identical). With increase of the incidence angle,
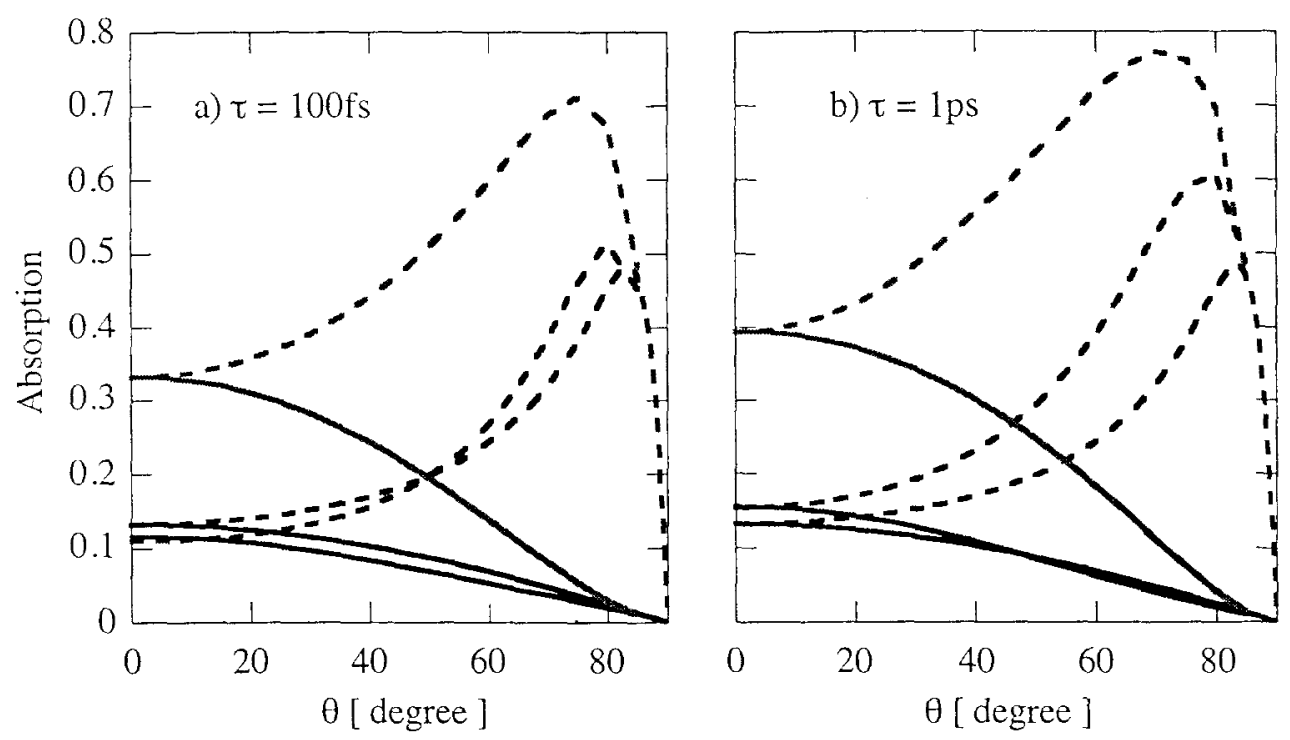

Figure 2.24: Plot of absorption versus incidence angle for $800 \mathrm{~nm}$ light and two different pulse lengths (100rs and $1 \mathrm{ps})$ and intensities $\left(10^{13} \mathrm{~W} / \mathrm{cm}^{2}\right.$ and $\left.10^{1+} \mathrm{W} / \mathrm{cm}^{2}\right)$. For reference absorption of cold aluminum calculated with Fresnel formulas using handbook value of refractive index [Palik98]. Results for $\mathrm{P}$ polarization are plotted with dashed lines. S polarization is solid lines. Black lines - Fresnel; blue - HYADES, $I=10^{13} \mathrm{~W} / \mathrm{cm}^{2}$; red HYADES, $I=10^{14} \mathrm{~W} / \mathrm{cm}^{2}$. 
absorption of the S polarized wave gradually decreases and goes to zero at 90 degrees. Behavior of the P polarized light is different. The maximum of absorption for the TM wave is located at some angle different from zero. Absorption at this maximum can be much higher than for normal incidence. In fact for materials with zero conductivity (dielectrics) there is an angle, called Brewster's angle

$$
\theta_{B}=\tan ^{-1}(n)
$$

for which there is no reflected wave at all - the light is completely "absorbed". For metals there is always some light reflected, however the peak absorption can be quite high almost $50 \%$ for aluminum. The refractive index of cold aluminum is very large: $\mathrm{n}(800 \mathrm{~nm})=2.8+i 8.45$, thus the maximum occurs at a large angle $\theta \sim \tan ^{-1}(|n|) \sim 80^{\circ}$.

All of these features are present in the absorption of ULSPS. The main difference caused by laser heating is in the general rescaling of absorption and broadening of the peak of absorption for $\mathrm{P}$ polarization. As a result, TM waves incident at high angles can deposit significant amounts of energy - absorption of $10^{14} \mathrm{~W} / \mathrm{cm}^{2}, 1 \mathrm{ps}$ pulse for angles between 60 and 80 degrees is above $70 \%$.

At high laser fluences or long pulses material expansion may begin to affect laser absorption since profile of dielectric function $\varepsilon(z)$ is no longer a step function. Reflection is determined by the gradient of permittivity $\partial \varepsilon / \partial z$ [Ginzburg55] and varies from $|(\sqrt{\varepsilon}-1) /(\sqrt{\varepsilon}+1)|^{2}$ for normal incidence on a flat surface, to zero when the gradient 
approaches zero. Theoretically, this should result in the increase of laser absorption through reduced reflectivity. To test this assumption pump-probe calculations were carried out. The pump is a rectangular laser pulse with $10^{14} \mathrm{~W} / \mathrm{cm}^{2}$ intensity and $100 \mathrm{fs}$ width. After the pump ablates the material, a low energy probe $\left(I=10^{5} \mathrm{~W} / \mathrm{cm}^{2}, \tau=10 \mathrm{fs}\right)$ at different delays is used to calculate absorption. The simulations were done for $45^{\circ}$ angle of incidence and $\mathrm{S}$ and $\mathrm{P}$ polarizations. Absorption of the pump is $21.6 \%$ for $\mathrm{S}$ polarization and $46.5 \%$ for $\mathrm{P}$ polarization. The result for absorption of the probe is presented in Figure 2.25. As we expected, increase in the delay between the pump and the probe results in larger absorption. For $15 \mathrm{ps}$ delay it is $85 \%$ for $\mathrm{S}$ and $95 \%$ for $\mathrm{P}$ polarizations.

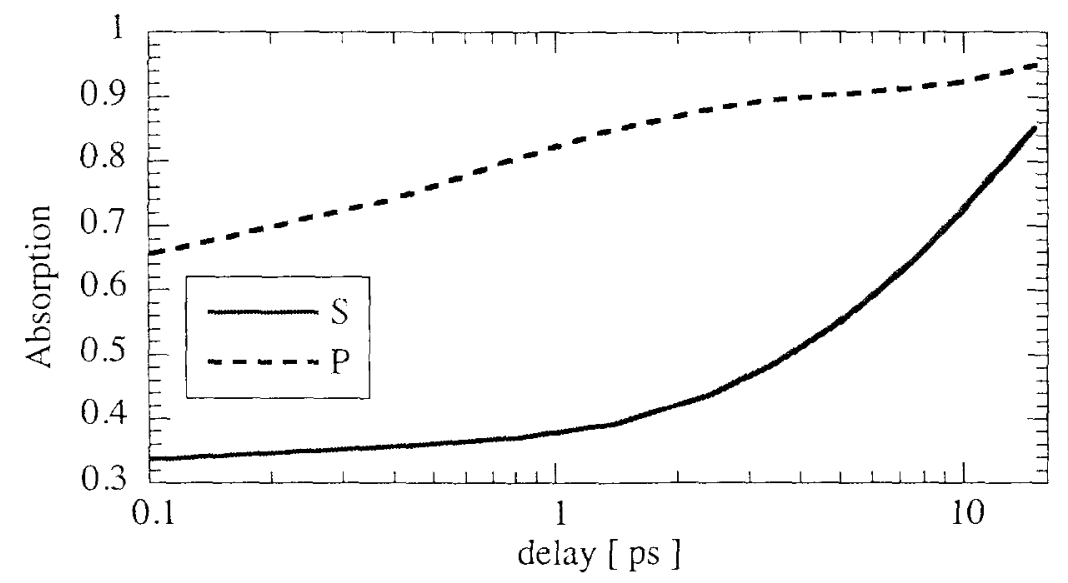

Figure 2.25: Plot of the probe absorption as a function of time delay after absorption of rectanguiar, $100 \mathrm{fs}, 10^{1.4} \mathrm{~W} / \mathrm{cm}^{2}$ pump pulse.

Figure 2.26 shows snapshots of laser power deposition density and electron density at several different delays for $\mathrm{S}$ polarized wave. $100 \mathrm{fs}$ after the pump the material 
expansion is negligible and change of absorption is mostly due to the high temperature. Most of the laser energy is deposited in a layer approximately $50 \mathrm{~nm}$ thick. Several picoseconds later the picture changes considerably. The ejecta plume thickness is hundreds of nanometers and, since the light wave can not propagate in plasma with electron density above critical $n_{c r}$, the peak of absorption shifts away from the original position of the material surface. If 3.4ps after the pump some of the laser energy does reach the bulk of the material, at 15 ps delay all of the laser energy is deposited in the expanding ejecta and may not reach the solid.

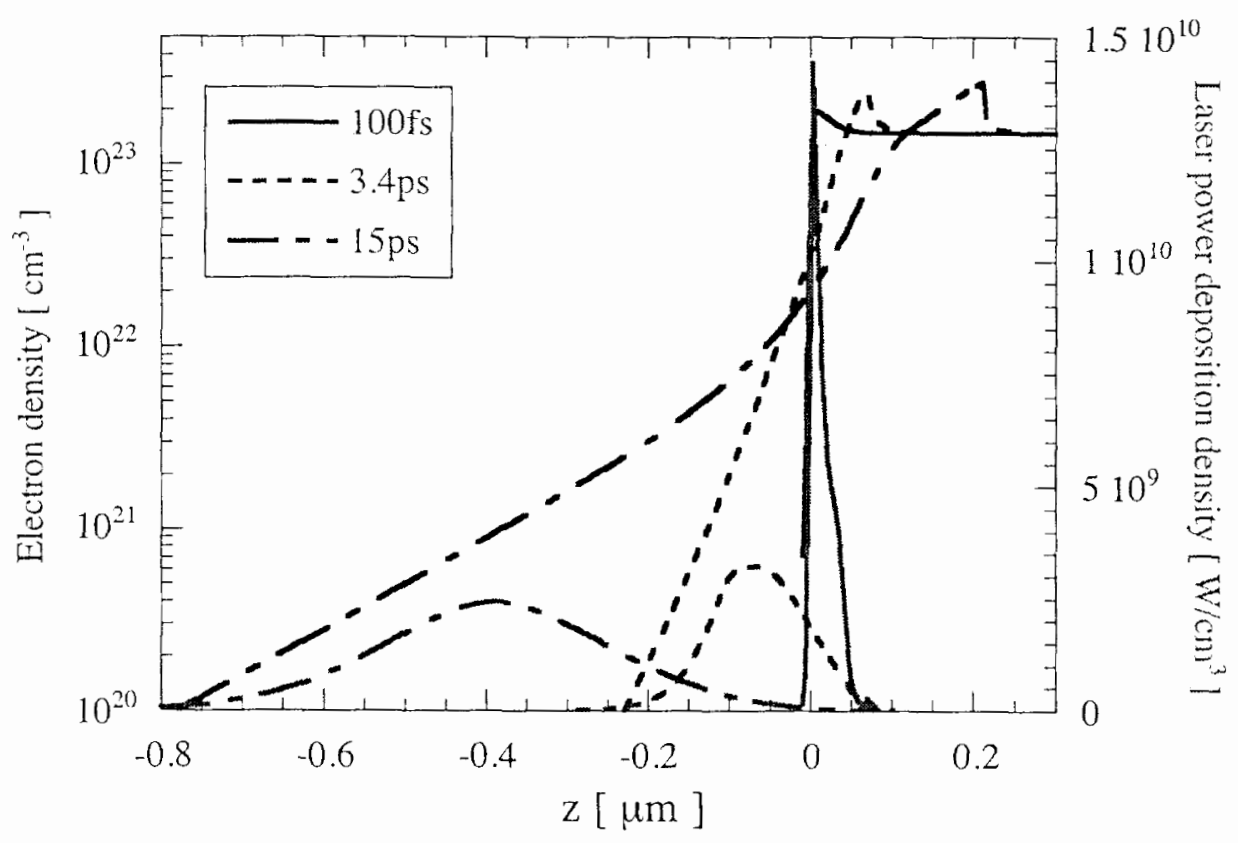

Figure 2.26: Snapshots of electron density (blue curves) and laser power deposition (red curves) for different delays after the rectangular, $100 \mathrm{fs}, 10^{14} \mathrm{~W} / \mathrm{cm}^{2}$ pump pulse. Pump and probe are $S$ polarized. Intensity of the probe is $10^{5} \mathrm{~W} / \mathrm{cm}^{2}$. Laser is incident from the left. Increase of electron density in the bulk of the material $(z>0)$ is due to the shock wave compression. 
The absorption picture is complicated by another fact. If the probe is energetic, it deposits energy, increases temperature and changes optical properties. For a probe that is identical to the pump $\left(I=10^{14} \mathrm{~W} / \mathrm{cm}^{2}, \tau=100 \mathrm{fs}\right)$ and delayed by $0.5 \mathrm{ps}$ absorption is $35 \%$ close to the results shown in Figure 2.25, however for a delay of 15 ps probe absorption is only $28.6 \%$. More than that, it was rapidly decreasing during the pulse and by its end it was $17 \%$ (versus $85 \%$ for low energy probe). The absorbed energy pumps up plasma temperature significantly and decreases collision frequency. We have plasma mirror effect [Perry99]. Therefore one can expect increase of absorption at first and then decrease. The details of interaction depend on many parameters such as energy of the pump and the probe, polarization, angle and can be calculated with HYADES.

These calculations also emphasize importance of a good laser pulse quality. In USLP laser systems there is a variety of effects, for example amplified spontaneous emission, regenerative amplifier leaks, spectral clipping [Backus98], that can create pulses before the main pulse. Even low energy prepulses can have noticeable effect on the interaction of the main pulse [Wharton01] and complicate interpretation of experiments. Thus, prepulse control is very important.

\subsection{Conclusion}

Our modeling of USLP interaction with NFE metals demonstrated several important physical aspects of the interaction. Unequal electron and ion temperature 
dynamics plays a central role in the interaction. Since the heat capacity of electrons in a metal is very small, even low intensity pulses quickly raise the electron temperature to thousands of degrees. Electron thermal transport can have noticeable effects, as a result the energy deposition zone is larger than the absorption zone. This, in turn, masks effects of different laser wavelength with the major difference being the amount of laser energy absorbed. Due to the significant imbalance in electron and ion temperatures, electronelectron collisions have a large (sometimes dominant) contribution to the total collision frequency. Influence of the interband transitions on optical properties is important even for an NFE metals like aluminum and may produce unusual results (lowering of absorptivity with temperature).

All of these effects were taken into account in the development of the hydrocode HYADES which is now suitable for self-consistent simulation of interaction of USLPS with NFE metals for a variety of laser parameters. The ability to simulate light absorption simultaneously with material expansion is important since material ejection may begin very quickly because of the significant electron pressure. Depending on the pulse energy and length, this expansion might enhance light energy deposition, however it also might happen outside of the bulk material, in the expanding ejecta.

The advantages of this approach (modeling on the basis of hydrocode) are not exhausted yet. Understanding of light absorption is typically an intermediate step. One is usually interested in long-term effects, for example material ablation or shock wave 
propagation. We demonstrated that the material expands with velocity on the order of $10^{7} \mathrm{~cm} / \mathrm{s}$, thus for a typical laser spot few hundred microns in size, calculations with $1 \mathrm{D}$ hydrocode are valid up to times on the order of few nanoseconds (before $3 \mathrm{D}$ effects become important). This long time modeling is discussed in the next chapter. 


\section{Chapter 3 Long-term effects of ultrashort laser-material interaction}

\subsection{Introduction}

Many desired effects of USLP laser-material interaction take place on a time scale noticeably longer than the interaction itself. For instance it takes over a hundred picoseconds for ablation generated pressure waves to travel a distance of only one micron; the ejecta plume used in thin film growth needs microseconds to reach a deposition target. This chapter describes our efforts to study the effects of ULSP absorption on nanosecond and longer time scales with the use of numerical and analytical modeling.

The chapter opens with a comparative, representative simulation of the effects of ultrashort (femtosecond) and short (nanosecond) laser pulse absorption. Differences in the absorption are emphasized. In the following section, we concentrate on the energy transport into the bulk of the material by the laser induced pressure pulses, namely shock waves. The pressure waves carry energy in a mechanical form (material compression and kinetic energy). We investigate conversion of this energy back into heat and show that this effect becomes significant for shock pressure in the hundreds of kilobars range and higher. Residual heating as a function of the shock pressure for aluminum and copper is 
calculated.

The next section is devoted to a study of evolution of the laser induced pressure pulses. The formation and propagation of the pressure pulses in different laser energy regimes is discussed. At low energy the effects of material strength are dominant and the pressure pulse has a smooth bipolar form. Increase in the laser fluence results in the fast formation of the shock wave and fast decay of the pressure pulse. If the pressure is very high (many megabars), the influence of material strength is negligible and the evolution of the shock wave is described by a self-similar solution for hydrodynamic motion of an ideal gas under action of an impulsive load. In this regime the wave parameters have a power law dependence on time and the shock pressure is proportional to the inverse distance traveled. Weaker waves decay more slowly; their asymptotic behavior is described by the square root of the inverse distance traveled.

After that our attention shifts to material ablation. Our goal is to find a criterion that would give us an estimate of the amount of the material removed based on the material evolution within the first nanosecond, so that the results of HYADES modeling could be used for the prediction. Structure of the ejecta plume is discussed and such a criterion based on the use of the liquid-vapor critical point is suggested. We apply it in a range of HYADES calculations and show that the most efficient removal is with femtosecond pulses near the ablation threshold.

The concluding part of this chapter presents discussion of the three-dimensional 
evolution of the ablation plume. In particular, a self-similar analytical solution for an expanding gas ellipsoid is used to describe plume properties at the final stage of evolution. The model, together with the results of HYADES modeling, is used to show that the ablation plume is very directional in its expansion.

\subsection{Effects of ultrashort and short pulse interaction}

In the introduction chapter a brief discussion of differences between ablation with short and ultrashort pulses was presented. One can actually see these differences in the

HYADES simulations - here we present the results of comparative modeling of ultrashort and short pulses interacting with aluminum.

Laser systems generating short ( 1ns) and ultrashort ( $\sim 1 \mathrm{ps})$ pulses work in very different regimes, therefore pulse parameters can not serve as a basis for comparison. A more proper way to do it is to compare ablation of pulses that create similar long-term effects, for example material removal. Let us go through the details of laser absorption and material ablation for one particular case: $350 \mathrm{fs}, 5 \mathrm{~J} / \mathrm{cm}^{2}$ and $1 \mathrm{~ns}, 20 \mathrm{~J} / \mathrm{cm}^{2}$ pulses at $1053 \mathrm{~nm}$ wavelength. Both of these pulses, when incident normally on aluminum, remove the same amount of material [Perry98]. In both cases material evolution up to $2.5 \mathrm{~ns}$ after the pulse peak was simulated. Figure 3.1 shows laser power deposition density as a function of time. As expected practically all of the USLP energy is absorbed in the solid material. There is some expansion during the pulses, but it seems to have insignificant 
effect. The absorption depth is approximately $25 \mathrm{~nm}$ - the pulse energy is high enough to affect optical properties.
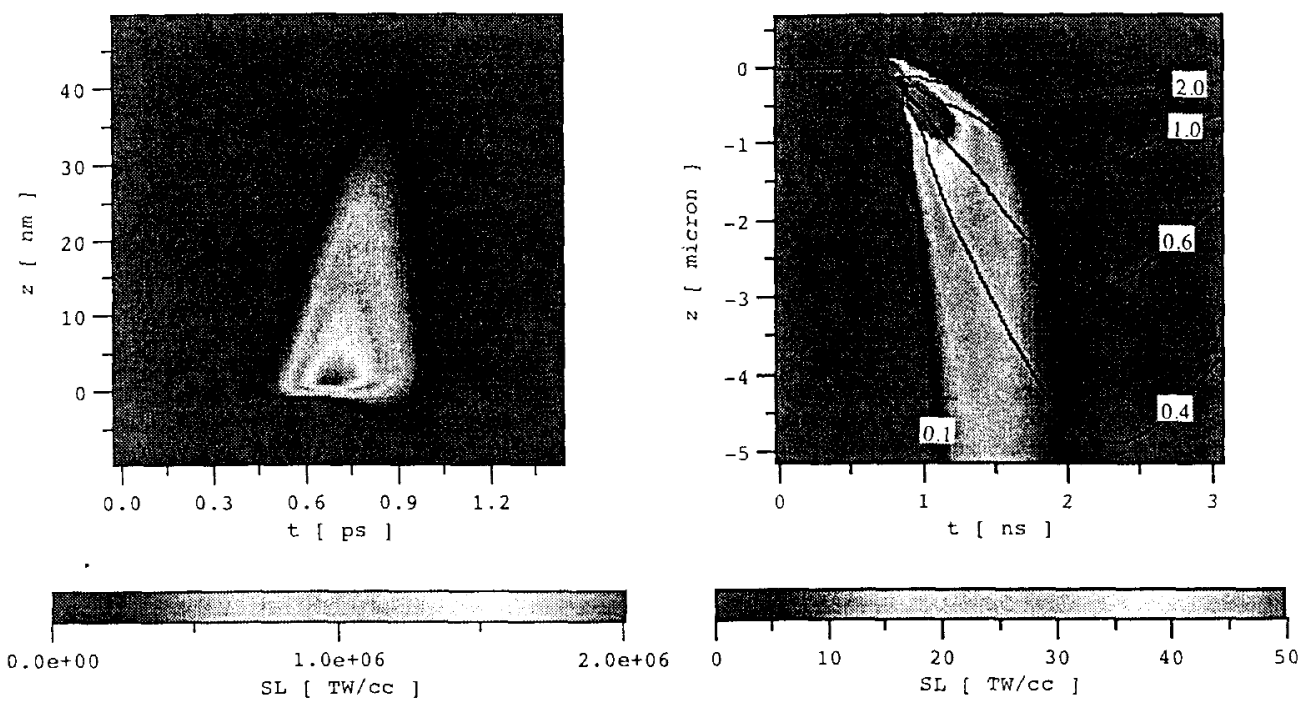

Figure 3.1: $(z, t)$ color plots of laser power deposition density for $350 \mathrm{fs}, 5 \mathrm{~J} / \mathrm{cm}^{2}$ (left) and $1 \mathrm{~ns}, 20 \mathrm{~J} / \mathrm{cm}^{2}$ (right) pulses. Notice the difference in spatial and temporal scales. Laser light is incident along the $\mathrm{z}$ axis and the material initially occupies region with positive $z$. Pulse peaks are at $700 \mathrm{fs}$ and $1.5 \mathrm{~ns}$ respectively. Contour lines on the plot for 1 ns pulse show electron density $N_{e} / N_{c r}$, where $N_{c r}$ is critical density for $1053 \mathrm{~nm}$ light $\left(\sim 10^{21} \mathrm{~cm}^{-3}\right)$.

For the nanosecond pulse, the absorption picture is completely different. Material expansion is quite noticeable. For a better demonstration of its effect, contour lines of the electron density were included in the plot. At the pulse peak $(t=1.5 \mathrm{~ns})$ the critical electron density is $1 \mu \mathrm{m}$ away from the original position of the material surface. Laser light can not penetrate deeper, in the denser region; thus practically all of the energy is absorbed in the expanding plume. This is a plasma shielding effect. In reality it might be even more noticeable, because HYADES does not include contribution to evaporation due to the 
kinetic effects (even if the material temperature is relatively low and there is no massive evaporation, some molecules from the energetic tail of the kinetic distribution have enough energy to overcome potential forces and leave the surface). Light absorption in such near critical density plasma is significant. Total absorption is $21.3 \%$ for the USLP and $93.2 \%$ for the nanosecond pulse (cold aluminum absorption at $1053 \mathrm{~nm}$ wavelength is 4.5\% [Palik98]). Another interesting property is radiation conversion efficiency. In the previous chapter we noticed it was very low in USLP ablation. Indeed, in this case $7.4 \cdot 10^{\circ}$ ${ }^{3} \%$ of 350 fs pulse energy is converted into radiation. On the contrary, for the nanosecond pulse it is $31.6 \%$. Conversion into radiation in the lower density plume is more efficient. Therefore, if one is interested in ultrashort X-ray sources, better conversion efficiency for USLP can be reached with a use of a prepulse that creates a dense plasma profile, where the main USLP would have higher $x$-ray generation [Nakano96,Nakano98].

Differences in absorption immediately appear in the behavior of temperature (shown in Figure 3.2). In the USLP case it peaks at approximately $6 \mathrm{eV}$. This hot material is quickly ejected and has no opportunity to transfer its energy into the bulk of the material. At $2.5 \mathrm{~ns}$ after the pulse peak, a surface layer with thickness about $0.7 \mu \mathrm{m}$ is heated to temperatures above melting. However, it is still relatively cold and heat penetration has slowed down. Higher beam energy and absorption of the nanosecond pulse create a plume with a noticeably larger temperature- above $15 \mathrm{eV}$, but, even more important is that the temperature is long lived. 

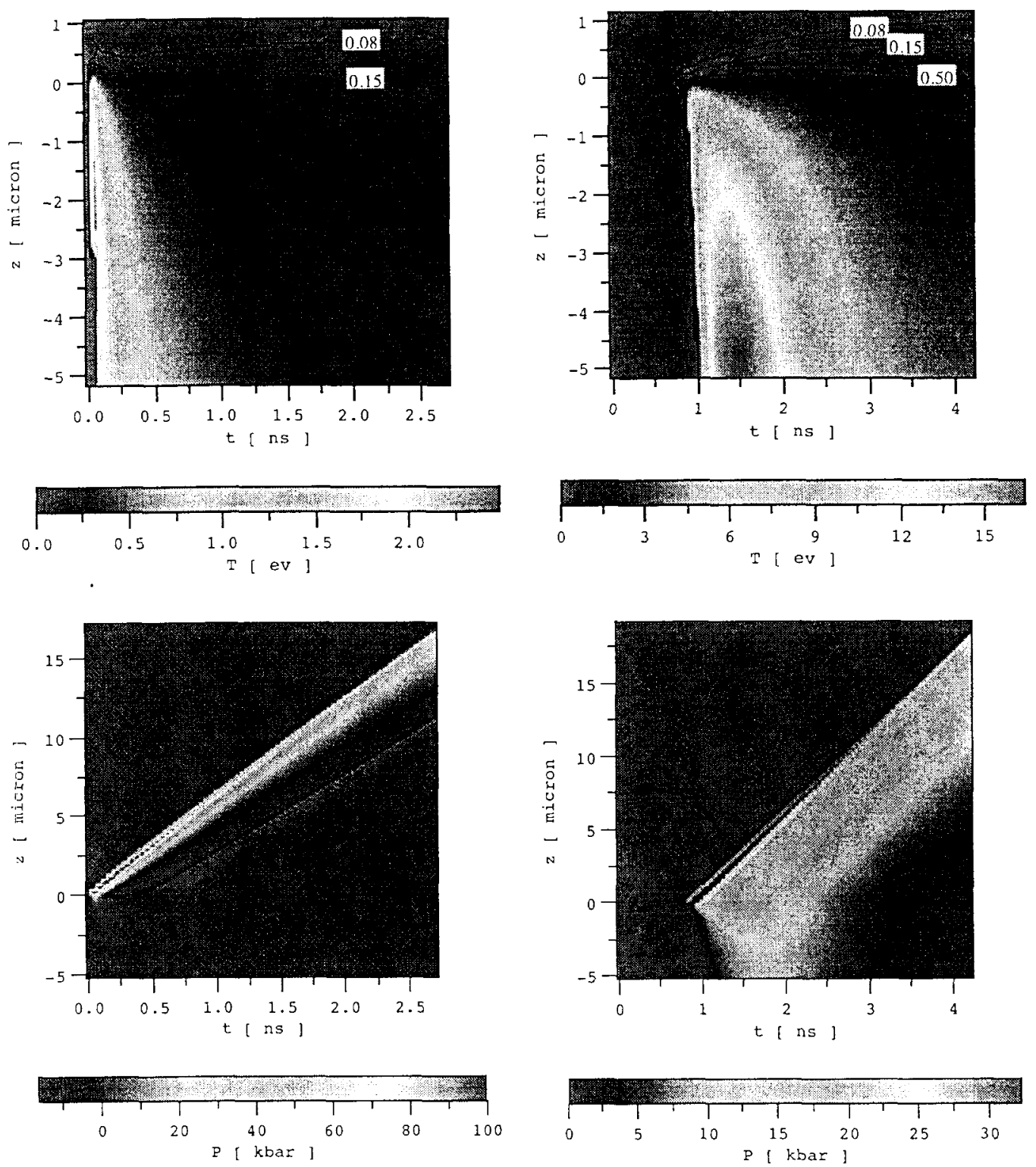

Figure 3.2: $(z, t)$ color plots of temperature (top) and pressure (bottom) for $350 \mathrm{fs}, 5 \mathrm{~J} / \mathrm{cm}^{2}$ (left) and $1 \mathrm{~ns}, 20 \mathrm{~J} / \mathrm{cm}^{2}$ (right) ablation. Laser light is incident along the $\mathrm{z}$ axis and the material originally occupies region with positive $z$.

Hot plasma stays near the solid aluminum for a longer time and much of its energy is transferred in the bulk. At $2.5 \mathrm{~ns}$ after the pulse peak, a surface layer with thickness $1 \mu \mathrm{m}$ 
has temperature above melting and the energy diffusion into the bulk is still significant.

USLP absorption occurs in a thin surface zone. High energy deposition creates high temperature and high pressure. In this case the peak pressure is almost $2 \mathrm{Mbar}$, however it decays quickly. When the pressure wave propagates into the bulk of the material, a number of processes, for instance rarefaction wave, decrease its amplitude. According to Figure 3.2 at Ins after the absorption the maximum pressure drops below $100 \mathrm{kbar}$ and continues decreasing. Pressure wave width is a few microns and the propagation speed is practically a constant close to the speed of sound. In the case of the nanosecond pulse, the pressure stays in the tens of kilobar range from the beginning. The pulse also propagates with speed close to that of sound; however its width is approximately ten microns. In nanosecond ablation, the pressure pulse temporal width is mostly determined by the width of the laser pulse. In the USLP case laser-material interaction is negligibly short when compared to characteristic hydrodynamic times. Thus the temporal pressure width, at least initially, is determined by the time it take sound speed to cross the surface heated zone.

\subsection{Energy transport and deposition by shock waves}

Laser ablation launches a pressure pulse that propagates into the bulk of the material. Speed of the propagation is determined by the speed of sound $c$ [Landau59, Zel'dovich69] 


$$
c^{2}=\left.\frac{\partial P(\rho, S)}{\partial \rho}\right|_{\text {enropy }}
$$

Since it is a function of material density (compression), the shape of the pressure pulse changes along the propagation path. For most solid materials, the speed of sound is higher when the density (pressure) increases. Thus, a smooth pressure pulse begins to steepen and finally a wave with sharp discontinuity is formed (see Figure 3.3). It is called a shock wave. In the case of USLP ablation it can form very quickly because initial pressure pulse is created in a thin surface zone and has a sharp profile from the

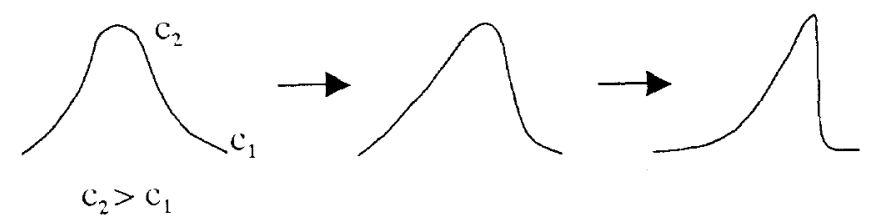

Figure 3.3: Shock wave formation from initially smooth pressure wave. For a typical material sound speed $c$ is an increasing function of pressure, thus higher pressure disturbances propagate faster, the profile steepens and shock wave forms.

beginning. Material parameters before and after the shock front are connected through the jump conditions that are derived from the equations of conservation [Landau59, Zel'dovich67]. Conservation of mass results in

$$
\rho_{0}\left(U_{s}-u_{0}\right)=\rho_{1}\left(U_{s}-u_{1}\right)
$$

momentum

$$
P_{1}-P_{0}=\rho_{0}\left(U_{S}-u_{0}\right)\left(u_{1}-u_{0}\right),
$$

and energy 


$$
E_{1}-E_{0}=\frac{1}{2}\left(P_{1}+P_{0}\right)\left(V_{0}-V_{1}\right)
$$

Subscripts 0 and 1 refer to the undisturbed material before the shock front and compressed material behind the shock. Parameters $u, \rho, P, E$ are material velocity, density, pressure and internal energy. $U_{S}$ is the shock speed. If the equation of state of the material is given together with, for instance, shock pressure, solution of the equations (3.2)-(3.4) defines a point in the space of thermodynamic variables that describes the compressed material. The locus of points obtained by shocking of a material in an initial state $\left(T_{0}, V_{0}\right)$ is called a Hugoniot. Figure 3.4 shows a schematic plot of Hugoniot for a typical material.

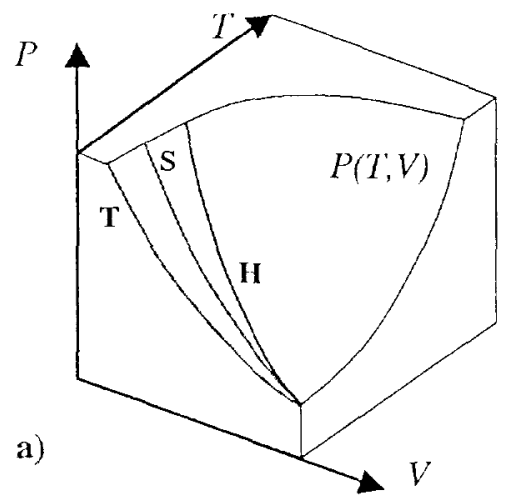

b)

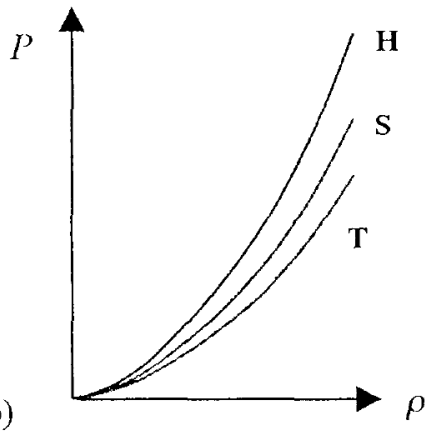

Figure 3.4: Schematic plot of isotherm (T), isentrope (S) and Hugoniot $(\mathrm{S})$ as a function of volume and temperature (a). Projection of the curves on $(P, \rho)$ plane is also shown (b). $P(V, T)$ is equation of state surface, where all curves lie.

The picture also shows curves for isothermal and isentropic (adiabatic) compression. 
While it is well known that isentrope differs from isotherm and adiabatic compression results in an increase of temperature, behavior of the Hugoniot is not obvious. Shock compression certainly involves material heating, however how it happens in relationship to the isentrope can be found using jump relationships [Landau59, Zel'dovich67].

Let us consider compression by a weak shock wave, such that all important parameters can be expanded in Taylor series around the initial state of the material. As independent variables we choose entropy $S$ and volume $V$. Expressing internal energy $E(S, V)$ and pressure $P(S, V)$ as a series expansions at $\left(S_{0}, V_{0}\right)$

$$
\begin{aligned}
& E_{1}=E_{0}+\left.\frac{\partial E}{\partial S}\right|_{V}\left(S_{1}-S_{0}\right)+\left.\frac{\partial E}{\partial V}\right|_{S}\left(V_{1}-V_{0}\right)+\ldots \\
& P_{1}=P_{0}+\left.\frac{\partial P}{\partial S}\right|_{V}\left(S_{1}-S_{0}\right)+\left.\frac{\partial P}{\partial V}\right|_{S}\left(V_{1}-V_{0}\right)+\ldots
\end{aligned}
$$

and substituting them in the jump condition (3.4), we can obtain a formula for entropy change along the Hugoniot in terms of thermodynamic derivatives and change in volume.

Carrying out the expansion up to third order and using formulas

$$
\left.\frac{\partial E}{\partial S}\right|_{V}=T,\left.\quad \frac{\partial E}{\partial V}\right|_{S}=-P
$$

The following relationship is obtained

$$
\begin{gathered}
\left.\frac{1}{12}\left(V_{1}-V_{0}\right)^{3} \frac{\partial^{2} P}{\partial V^{2}}\right|_{S}+T_{0}\left(S_{1}-S_{0}\right)+\left.\frac{1}{2}\left(S_{1}-S_{0}\right)\left(V_{1}-V_{0}\right) \frac{\partial P}{\partial S}\right|_{V}+\left.\frac{1}{2}\left(S_{1}-S_{0}\right)^{2} \frac{\partial^{2} E}{\partial S^{2}}\right|_{V}+ \\
\left.\frac{1}{4}\left(S_{1}-S_{0}\right)^{2}\left(V_{1}-V_{0}\right) \frac{\partial^{2} P}{\partial S^{2}}\right|_{V}+\left.\frac{1}{6}\left(S_{1}-S_{0}\right)^{3} \frac{\partial^{3} E}{\partial S^{3}}\right|_{V} \approx 0
\end{gathered}
$$


If not for the first term, solution of this equation could have been obtained by setting $S_{\text {I }}$ equal to $S_{0}$. In other words when volume change is small (weak shocks), Hugoniot is almost an isentrope. Entropy variation along the Hugoniot is approximately a third order function of change in volume

$$
S_{1}-S_{0}=\left.\frac{1}{12 T_{0}} \frac{\partial^{2} P}{\partial V^{2}}\right|_{S}\left(V_{0}-V_{1}\right)^{3}
$$

Behavior of the pressure derivative can be related to the change of sound speed with compression

$$
\frac{\partial c^{2}}{\partial \rho}=2 c \frac{\partial c}{\partial \rho}=\left.\frac{\partial^{2} P}{\partial \rho^{2}}\right|_{S}=\frac{\partial}{\partial \rho}\left(-\left.\frac{1}{\rho^{2}} \frac{\partial P}{\partial V}\right|_{S}\right)=\frac{1}{\rho^{3}}\left(\left.2 \frac{\partial P}{\partial V}\right|_{S}+\left.\frac{1}{\rho} \frac{\partial^{2} P}{\partial V^{2}}\right|_{S}\right)
$$

Shock waves form when the sound speed increases with pressure(density). Since pressure in a typical material drops with increase of volume, this condition is satisfied only when the second volume derivative is non-zero and positive. Therefore if the shock wave does form, it increases material entropy.

Relative pressure change can be deduced from the series expansion

$$
P_{1}-P_{0}=\left.\frac{\partial P}{\partial V}\right|_{S}\left(V_{1}-V_{0}\right)+\left.\frac{1}{2}\left(V_{1}-V_{0}\right)^{2} \frac{\partial^{2} P}{\partial V^{2}}\right|_{S}+\left.\frac{1}{6}\left(V_{1}-V_{0}\right)^{3} \frac{\partial^{3} P}{\partial V^{3}}\right|_{S}+\left.\frac{\partial P}{\partial S}\right|_{V}\left(S_{1}-S_{0}\right)
$$

Since the entropy change is third order function of volume change, only one entropy term is left. According to this formula, Hugoniot and isentrope have the same slope and curvature initially. The higher order difference is described by the last term. With the use of formula (3.7) and expression for heat capacity 


$$
C_{V}=\left.\frac{\partial E}{\partial T}\right|_{V}=\left.T \frac{\partial S}{\partial T}\right|_{V}
$$

it can be written as

$$
\begin{gathered}
P_{H}-P_{S}=\left.\frac{\partial P}{\partial S}\right|_{V}\left(S_{1}-S_{0}\right)=\left.\left.\frac{1}{12 T_{0}} \frac{\partial P}{\partial S}\right|_{V} \frac{\partial^{2} P}{\partial V^{2}}\right|_{S}\left(V_{0}-V_{1}\right)^{3}= \\
=\left.\left.\frac{1}{12 C_{V}} \frac{\partial P}{\partial T}\right|_{V} \frac{\partial^{2} P}{\partial V^{2}}\right|_{S}\left(V_{0}-V_{1}\right)^{3}
\end{gathered} .
$$

Since the pressure typically increases when the material is heated at constant volume, derivative $\partial P /\left.\partial T\right|_{V}$ is positive. Thus pressure on the Hugoniot increases faster than on the isentrope. Once the shock has passed, a rarefaction wave sets in and the compressed material begins to expand adiabatically along the isentrope. In this case, the pressure drops slower than on the Hugoniot. If we follow the material state as the shock wave comes and leaves, the following scenario emerges. When the material is shocked, its pressure and entropy increase along the Hugoniot. In the rarefaction stage, the pressure drops along the isentrope. However, the pressure decrease is not as fast as during the compression, thus, when it reaches original volume (density) $V_{0}$, the pressure is higher than $P_{0}$. This is equivalent to saying that the final temperature is higher than at the beginning, before the shock wave came. The increase in internal energy comes from the shock wave energy. Therefore, even in the absence of thermal diffusion, there would be heating by shock waves. This may have a significant impact because hydrodynamic motion is one of the fastest processes in the laser ablation. 
In order to estimate how significant this effect is, we calculated shock wave pressure needed to start melting of aluminum and copper. We begin by deriving expressions for temperature evolution along the isentrope and Hugoniot.

Let us consider the thermodynamic identity

$$
T d S=d E+P d V
$$

The expression can be simplified, if we consider Helmholtz free energy of the solid material [Landau69]

$$
F=E_{c}(V)+3 N k_{B} T \ln \left(x \frac{\theta_{D}}{T}\right)
$$

The first term is the contribution of interparticle interactions, it is a function of distance between molecules, and thus it depends on volume only. The second term is the contribution of phonons that are considered in a high temperature limit: as a collection of classical oscillators with constant heat capacity and typical frequency $x k_{B} \theta_{D} / \hbar$, where $x$ is a numerical coefficient. The thermal contribution of electrons can be omitted for moderate temperatures. Internal energy and pressure are calculated as

$$
E=F-T \frac{\partial F}{\partial T}, \quad P=-\frac{\partial F}{\partial V}
$$

Thus the energy is

$$
E=E_{c}(V)+3 N k_{B} T=E_{c}(V)+C_{V} T=E_{c}(V)+E_{T}(T)
$$

and pressure 


$$
P=-\frac{\partial E_{c}}{\partial V}-\frac{3 N k_{B} T}{\theta_{D}} \frac{\partial \theta_{D}}{\partial V}=-\frac{\partial E_{c}}{\partial V}-\frac{3 N k_{B} T}{V} \frac{\partial \ln \left(\theta_{D}\right)}{\partial \ln (V)}=P_{c}(V)+\frac{\Gamma(V)}{V} E_{T}(T),
$$

where coefficient $\Gamma(V)$

$$
\Gamma(V)=\frac{\partial \ln \left(\theta_{D}\right)}{\partial \ln (V)}
$$

is called the Grüneisen gamma. It is a weak function of volume and for many metals its value is close to two. Substituting expressions (3.13),(3.14) into (3.10) we get

$$
d S=\frac{1}{T}(d E+P d V)=\frac{1}{T}\left(d E_{T}+P_{T} d V\right)=C_{V} \frac{d T}{T}+\Gamma C_{V} \frac{d V}{V} .
$$

The differential equation that describes temperature change along the isentrope $(d S=0)$ immediately follows:

$$
\left.\frac{d T}{d V}\right|_{S}=-\Gamma(V) \frac{T}{V}
$$

Solution of these equations depends on the particular model used for the Grüneisen gamma. There are a variety of experimental models. The simplest one is to choose a constant Grüneisen gamma. Another popular choice is to set it proportional to the volume

$$
\Gamma(V)=\Gamma_{0} \frac{V}{V_{0}}
$$

where $V_{0}$ is a reference volume at normal conditions. We use a linear model of gamma [Royce71]

$$
\Gamma(V)=\Gamma_{0}+a \frac{V-V_{0}}{V_{0}},
$$

since there is data availability for many materials [Steinberg91]. Parameters $\Gamma_{0}$ and $a$ are 
measured experimentally [Steinberg91].

In this case the solution of equation (3.17) is

$$
T(V)=T\left(V_{0}\right) \exp \left(-\int_{V_{0}}^{V} \frac{\Gamma(x) d x}{x}\right)=T\left(V_{0}\right) \exp \left(-a \frac{V-V_{0}}{V_{0}}\right)\left(\frac{V_{0}}{V}\right)^{r_{0}-a}
$$

The formula describes change of temperature when material is compressed or expanded adiabatically. Next we are going to derive formula for temperature change along the Hugoniot.

Using identity (3.10) on Hugoniot, we obtain

$$
\left.T \frac{d S}{d V}\right|_{H}=\left.\frac{d E}{d V}\right|_{H}+P_{H}
$$

Substituting from jump condition (3.4), the expression for internal energy as a function of pressure and neglecting initial pressure $P_{0}$, the formula for entropy change is simplified to

$$
\begin{gathered}
\left.T \frac{d S}{d V}\right|_{H}=\left.\frac{d}{d V}\left(E_{0}+\frac{1}{2}\left(P_{H}+P_{0}\right)\left(V_{0}-V\right)\right)\right|_{H}+P_{H}= \\
=\frac{1}{2}\left(P_{H}+\left.\left(V_{0}-V\right) \frac{d P}{d V}\right|_{H}\right)
\end{gathered}
$$

Formula for the shock pressure as a function of volume can be derived for materials that have available experimental data on measurement of shock velocity versus particle velocity behind the shock front (equivalent to measurement of the equation of state). This data often conforms with high accuracy to the equation

$$
U_{\text {shock }}=A+b u_{1},
$$

where $A$ and $b$ are material specific coefficients [Kinslow70,Marsh80,Steinberg91] 
(initial particle velocity $u_{0}$ is zero). Using jump relationship (3.2) and (3.3) and the equation for shock velocity (3.22) the following formula for Hugoniot pressure is derived

$$
P_{H}=\frac{\rho_{0} A^{2} \eta}{(1-b \eta)^{2}}
$$

where parameter $\eta=1-V / V_{0}$. Substituting this expression in (3.21), we get

$$
\left.T \frac{d S}{d V}\right|_{H}=-\frac{\rho_{0} A^{2} b \eta^{2}}{(1-b \eta)^{3}}=f(V)
$$

From equation (3.16), the entropy change along the isentrope can be also expressed in terms of heat capacity and the Grüneisen gamma. Combining these equations we derive a differential equation that describes temperature change along the Hugoniot

$$
\left.\frac{d T}{d V}\right|_{H}+\frac{\Gamma(V)}{V} T=\frac{f(V)}{C_{V}}
$$

This is just an inhomogeneous version of equation (3.17). Solution of (3.25) is

$$
\begin{gathered}
T(V)=T\left(V_{0}\right) \exp \left(-\int_{V_{0}}^{V} \frac{\Gamma(x) d x}{x}\right)+\exp \left(-\int_{V_{0}}^{V} \frac{\Gamma(x) d x}{x}\right) \int_{V_{0}}^{V}\left[\exp \left(\int_{V_{0}}^{x} \frac{\Gamma(z) d z}{z}\right)\right] \frac{f(x) d x}{C_{V}}= \\
=\left[T\left(V_{0}\right)+\delta T(V)\right] \exp \left(-a \frac{V-V_{0}}{V_{0}}\right)\left(\frac{V_{0}}{V}\right)^{\Gamma_{0}-a}
\end{gathered}
$$

where function $\delta T$ is

$$
\delta T(V)=\frac{A^{2} b}{C_{V}} \int_{\frac{V}{V_{0}}}^{1} \exp (a(x-1)) \frac{(1-x)^{2} x^{\Gamma_{0}-a}}{(1-b(1-x))^{3}} d x
$$

When the compressed material expands adiabatically, its temperature evolution is described by formula (3.19). Comparing (3.19) and (3.26) we can see that $\delta T(V)$ is the 
residual increase of material temperature after going through compression and relaxation by the shock wave.

Before proceeding with the calculations of heating by a shock, we need to derive a formula describing the melting temperature as function of volume. It will allow us to find the location of the liquid metal region relatively to Hugoniot and isentropes. Lindemann [Mott58, Iida88] suggested that the following law describes the material melting

$$
T_{m}=\alpha \theta_{D}^{2} V^{2 / 3}
$$

where $\alpha$ is material specific constant. The reasoning behind this formula is quite simple. Lindemann postulated that melting occurs when the amplitude $x$ of atomic oscillations in the lattice reaches a certain fraction of the interatomic distance $X$. The average potential energy of an oscillator is [Landau76]

$$
M \omega^{2}\left\langle x^{2}\right\rangle \sim T
$$

The oscillation frequency $\omega$ is proportional to the Debye frequency $\theta_{D}$. Interatomic distance can be expressed as $V^{2 / 3}$, thus the formula (3.28) appears. Taking the derivative of the melting temperature with respect to volume, we obtain the differential equation

$$
\frac{d T_{m}}{d V}=\left(\frac{2}{3}-2 \Gamma(V)\right) \frac{T}{V}
$$

Solving this for our particular model of gamma (3.18), an expression for the melting temperature as a function of volume is derived 


$$
T(V)=T_{m 0} \exp \left(-2 a \frac{V-V_{m 0}}{V_{m 0}}\right)\left(\frac{V_{m 0}}{V}\right)^{2\left(\Gamma_{0}-a\right)-2 / 3} .
$$

When the material is heated, its equilibrium volume is slightly larger due to thermal expansion. We need to include this factor by employing formula

$$
V(T)=V_{0}+\beta\left(T-T_{0}\right),
$$

where $\beta$ is coefficient of volume expansion.

Table 3.1 lists parameters needed for our calculations for aluminum and copper.

\begin{tabular}{|c|c|c|c|c|c|c|c|c|}
\hline & $\begin{array}{c}\rho_{0}, \\
\mathrm{~g} / \mathrm{cm}^{3}\end{array}$ & $\Gamma_{0}$ & $a$ & $\begin{array}{c}A \\
\mathrm{~cm} / \mathrm{s}\end{array}$ & $B$ & $\begin{array}{c}T_{\operatorname{mn} \theta}, \\
\mathrm{K}\end{array}$ & $\begin{array}{l}\beta, \\
K^{-1}\end{array}$ & $\begin{array}{l}C_{v}, \\
\mathrm{~J} / \mathrm{gK}\end{array}$ \\
\hline $\mathrm{Al}$ & 2.7 & 1.97 & 1.5 & $5.34 \cdot 10^{5}$ & 1.34 & 930 & $8.15 \cdot 10^{-5}$ & 0.844 \\
\hline $\mathrm{Cu}$ & 8.93 & 2.02 & 1.5 & $3.94 \cdot 10^{5}$ & 1.489 & 1356 & $6.6 \cdot 10^{-5}$ & 0.37 \\
\hline
\end{tabular}
Initial temperature was set to room temperature $293 \mathrm{~K}$.

Table 3.1: Aluminum and copper parameters needed for calculation of material heating by a shock wave. The data is from [Gray63,Steinberg91].

Figure 3.5 shows calculated temperature on Hugoniot and isentropes as a function of aluminum density. Melting curve and equilibrium curves at atmospheric pressure are also included. When the material is shocked, its temperature increases along the Hugoniot. For density increase up to $10 \%$ it is practically indistinguishable from an isentrope, however once the density is $20 \%$ above normal, temperature on the Hugoniot begins to increase much faster. When the shocked density reaches approximately 
$310 \mathrm{ps}$ there are three zero velocity zones. They correspond to the initial depths of $40 \mathrm{~nm}$, $44.6 \mathrm{~nm}, 500 \mathrm{~nm}$ and $39 \mathrm{~nm}, 295 \mathrm{~nm}, 1300 \mathrm{~nm}$ respectively. The material velocity changes its sign several times. The explanation for this behavior can be found if we compare the velocity plot (Figure 3.14) with the pressure plot (Figure 3.10) for the same case. The positive velocity spike at large depth is associated with the shock wave. Once the compressed material expands, material velocity drops and changes its sign.

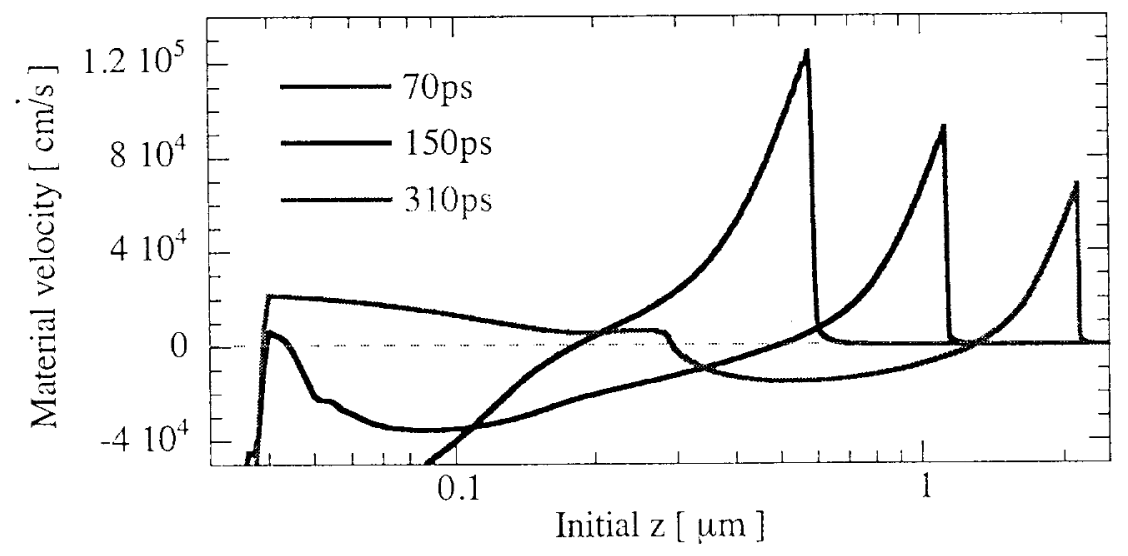

Figure 3.14: Plots of material velocity versus initial coordinate 70ps, 150fs and 310ps after the ablation with $800 \mathrm{~nm}, 100 \mathrm{fs}$, normally incident laser pulses with $2.5 \mathrm{~J} / \mathrm{cm}^{2}$ fluence.

However the temperature behind the compressive pulse is low and the density is close to the solid density. Thus a negative pressure appears and begins to decelerate the outward flow. In this case it stops the material completely and the second zero velocity zone appears. Since there is an expanding plume, the velocity passes through zero again and there are three zero velocity zones in total. The first two zones appeared because of the 
action of the material strength, thus it is reasonable to assume that the removal estimate is given by the last one. It is encouraging to see that from $150 \mathrm{ps}$ to $310 \mathrm{ps}$ the value practically doesn't change and stays at approximately $40 \mathrm{~nm}$. Also, it is below our upper estimate of $153 \mathrm{~nm}$.

The zero velocity zone criterion seems to be reasonable. However, it doesn't give enough physical insight. Thus, the results for each run must be visually inspected and one has to use other factors in deciding which of the zones to use to estimate the removal. The answer to our problem is hidden in the irregular behavior of the material density and temperature that can be seen in Figure 3.13. The curves for 150ps and 310ps look like there is some sort of numerical instability around $z \sim 100-200 \mathrm{~nm}$. The parameters experience significant fluctuations. The density drops from solid-like values to close to zero and then jumps back and this can happen several times. We are observing the effects of the liquid-vapor phase transition and the density fluctuations seen are caused by a physical instability. To understand these effects lets review an equation of state in this region. This will also help us to introduce a better criterion for the estimate of the material removal.

Pressure of an ideal gas is described by the equation

$$
P=\frac{N k_{B} T}{V},
$$

where $N$ is the number of particles in the volume $V$. In ideal gases, the influence of the 
potential interaction between atoms and molecules is very weak. However, when the gas temperature drops or volume decreases, we can no longer neglect potential forces. Thus the equation of state must be modified. If the gas is not very dense, the formula for the pressure can be expressed as an expansion in terms of $1 / V$ [Landau59]

$$
P=\frac{N k_{B} T}{V}\left(1+\frac{N B(T)}{V}+\frac{N^{2} C(T)}{V^{2}}+\ldots\right)
$$

The coefficients $B(T), C(T), \ldots$ are called virial coefficients and describe the effects of collisions in groups of two, three, four etc. molecules. Virial coefficients are material specific and can be expanded in terms of $1 / T$. Keeping only the first two terms, the approximation for $B(T)$ becomes [Landau59]

$$
B(T)=b-\frac{a}{k_{B} T}
$$

where coefficients $b$ and $a$ are positive. The first term describes the fact that the molecule occupies a finite volume ( $b$ is an effective volume of the molecule). The second term has a negative sign and describes the effect of the potential attraction that causes the matter to condense and become liquid when the temperature decreases. Neglecting higher order virial coefficient and substituting expression (3.45) in (3.44) we arrive at

$$
\left(P+\frac{N^{2} a}{V^{2}}\right)\left(\frac{V^{2}}{V+N b}\right)=N k_{B} T .
$$

In this approximation the volume occupied by the gas is much larger than the total volume occupied $N b$ so the expression in second parenthesis on the right hand side can be 
expanded. We finally obtain

$$
\left(P+\frac{N^{2} a}{V^{2}}\right)(V-N b)=N k_{B} T
$$

This is van der Waals' equation of state [Landau59] that provides a correct qualitative description of the material behavior in the liquid-gas region. There are many equations of this type; this is one of the simplest.

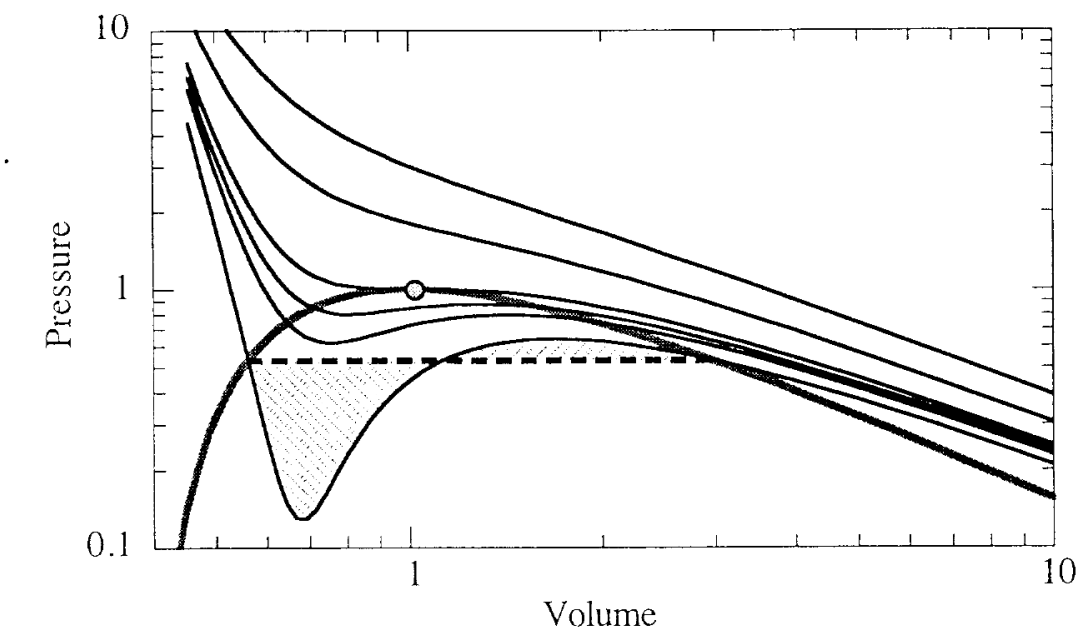

Figure 3.15: Plot of van der Waals isotherms (black solid curves). The orange dot is the liquid-vapor critical point. Green curve is the binodal - the boundary of the liquid-vapor coexistence region. The pressure in this region does not depend on volume (black dashed line) and it is calculated in such a way that the two shaded areas are equal (the so-called Maxwell's construction). In the picture the areas don't look equal only because it is a $\log$ log plot.

Liquid and gas (vapor) are two distinct phases of matter. However there is nothing in the equation (3.47) that distinguishes between them. Further analysis is needed for proper description of the phase transition. Figure 3.15 shows a plot of the van der Waals 
pressure isotherms. There are two types of curve. One corresponds to a high temperature, when the pressure decreases monotonically with increase in volume. The second appears when the temperature is below some critical temperature. In this case two pressure extrema are present. When the material expands, the pressure initially drops, then increases and then drops again. However the region

$$
\frac{\partial P}{\partial V}>0\left(\frac{\partial P}{\partial \rho}<0\right)
$$

can not be realized by matter in homogeneous equilibrium. In other words, it can not be represented by one material phase. The reason is quite simple. Suppose such a state exists. When a fluctuation of material density appears (they are always present due to the statistical nature of the problem), the pressure increases in the less dense region. Simultaneously the density of the neighboring material increases and the pressure drops. This pressure difference stimulates further expansion of the less dense part; thus such a state is unstable. The expansion stops when an equilibrium state different from the one described by van der Waals equation is reached. At this point the material is a liquidvapor mixture.

The process of finding a new equilibrium state in the mixed phase region is called Maxwell's construction [Landau59]. The pressure in this region is a constant function of volume and its value $P_{\text {aq }}$ for a particular temperature is found from the equation 


$$
\int_{V_{1}}^{V_{2}} P_{v d d V} d V=P_{e q}\left(V_{2}-V_{1}\right)
$$

Derivation of this equation is based on the condition that the chemical potential of the system is constant [Landau59]. Volumes $V_{1}$ and $V_{2}$ that designate the boundary of the liquid-vapor region are also found from this equation. The boundary of the mixed phase region is called the binodal. In Figure 3.15 , it is shown as the green curve. The point where the two sides of the binodal come together is called the liquid-vapor critical point (shown as an orange dot in Figure 3.15). Material parameters that correspond to this point are called critical, for instance, the critical temperature. In is often convenient to renormalize all the parameters in terms of their critical numbers. Therefore van der Waals equation becomes

$$
\left(P^{\prime}+\frac{3}{V^{\prime 2}}\right)\left(3 V^{\prime}-1\right)=8 T^{\prime}
$$

where $P^{\prime}=P / P_{c}, V^{\prime}=V / V_{c}, T^{\prime}=T / T_{c}$. The units in Figure 3.15 are normalized. If the material temperature is above critical, there is a smooth transition from liquid to vapor during the expansion. If the temperature is below critical, a new vapor phase appears in the form of bubbles upon crossing the binodal. As the volume increases, the fraction of the material in the gas form increases too and, when it crosses the binodal for the second time, it is completely vapor (here we use terms vapor and gas interchangeably. Typically a nonideal gas is meant by vapor). 
We have to notice that the mixed phase region is larger than the unstable region $\partial P / \partial V>0$. The boundary of the unstable region is called the spinodal. This curve lies inside of the mixed phase region and by definition is a locus of points $\partial P / \partial V=0$. It touches the binodal only in one point - critical point. Since these two regions are not the same, during fast volume change a material can follow the van der Waals EOS inside the mixed phase region and continue to be homogeneous, for instance, liquid. The expansion is fast, thus there is no time to settle on the equilibrium liquid-vapor state. We have either superheated liquid or supercooled vapor. When the spinodal is reached, the physical instability quickly creates phase separation. However, if the liquid temperature is not very high, the pressure can become negative before reaching the spinodal (for van der Waals EOS this happens if $T<0.85 T_{c}$ ). Superheated liquid exhibits tensile strength and the expansion can be stopped. This is called a metastable state. The expansion can continue if the material transforms from a liquid, metastable state to a stable, liquid-vapor mixed state. However, the expansion speed becomes noticeably smaller, since the sound speed drastically decreases when gas phase appears (sound speed in gas is mach smaller than sound speed in liquids and solids [Gray63]).

To see how these effects are relevant to the USLP material removal, let's follow the evolution of the material state on the schematic $(T, \rho)$ phase diagram (Figure 3.16). The diagram shows us the qualitative behavior of the material heated by different energy 


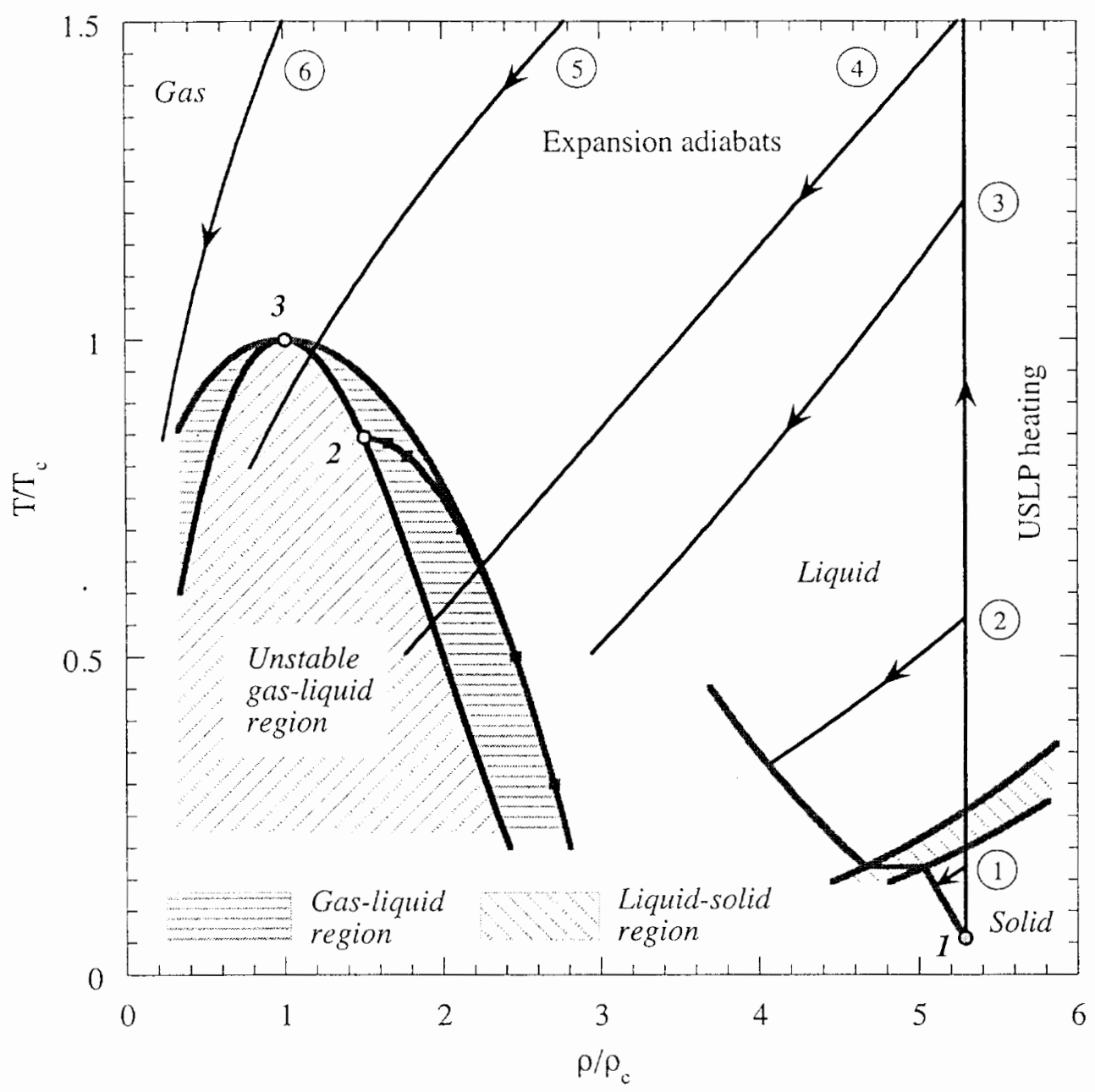

Figure 3.16: Schematic $(T, \rho)$ phase diagram describing material evolution in USLP ablation. Temperature and density are expressed in term of their critical values. Gas-liquid region is calculated with van der Waals equation. Green line bounding gas-liquid region is the binodal; blue line is the spinodal; red line with square markers is $P=0$ curve. Solid red line correspond to a material state with $P=1$ bar (material expansion at normal pressure). Green lines show boundaries of the liquid-solid region. Solid black lines show isochoric $(V, \rho=$ const) USLP heating and the expansion adiabats. Orange dots are 1 : normal state (room temperature, $P=1$ bar); 2 : point where negative pressure appears outside of the unstable region of the van der Waals equation; 3: critical point. Numbers in circles are explained in the text. 
USLPs. Before the absorption, the material is in the normal state (orange dot \#1): room temperature and normal density ( $P=1$ bar; Obar if in vacuum). For aluminum, critical parameters are $T_{c}=5700 \mathrm{~K}, \rho_{c}=0.51 \mathrm{~g} / \mathrm{cm}^{3}$ [Sesame83], thus the normal parameters are $\rho_{d} \rho_{c} \sim 5.2, T_{d} / T_{c} \sim 0.052$. For simplicity, we assume USLP heating to be isochoric ( $V, \rho=$ const; expansion during the absorption and shock compression is neglected). The expansion begins after the pulse is over. If we neglect thermal transport, the expansion proceeds along the material isentropes. Numbers in circles show different expansion adiabats. The first adiabat would correspond to a case similar to that described in Figure 2.18, Figure 3.7 and Figure 3.12: low fluence absorption. Peak temperature is very low and the material always remains solid. Upon the expansion it reaches a new equilibrium state located on the red curve (equilibrium at $P=1$ bar, if in vacuum $-P=0$ ) and the expansion stops. In the absence of spallation there is no material removal. This is the case when a bipolar pressure pulse is launched. Higher fluence absorption is described by the curve number 2 . The material is melted during the heating and the equilibrium point is also in the liquid phase. Since the temperature is rather low, there should be no material removal (unless there is a hydrodynamic instability that distorts the material surface and melt splashing appears). Adiabat number 3 comes into a region where the material expanding at the fixed pressure (red curve) reaches the binodal. We don't see this in the diagram because the gas-liquid region was calculated with van der Waals equation and 
the normal expansion curve is calculated from the experimental behavior of aluminum. Obviously this is a region where an equation of state better than van der Waals EOS must be used. The point where the lbar expansion curve (red solid line) reaches the binodal corresponds to a material boiling at $\mathrm{I}$ bar. For aluminum this happens at $T=2540 \mathrm{~K} \sim 0.45 T_{c}$ [Gray63]. If we use an equation of state without the Maxwell's construction, the expansion stops and there is no removal. Higher laser energy corresponds to the curve number 4 . It still reaches the binodal in the region where the liquid can withstand some tension: In order for the expansion to be stopped, the material must go inside the mixed phase region, where negative pressure appears (this works if the material is in the metastable state). If the expansion velocity is high, the material can reach the spinodal and gas phase may appear. Expansion curve number 5 reaches the gas-liquid region below the critical point, but above the point where the material can develop negative pressure. Thus it goes straight in the unstable region bounded by the spinodal. Once it is there, the physical instability breaks the homogeneous liquid into a mixture of gas and liquid. Effects of this instability can be seen in Figure 3.13. Material heated to an even higher temperature (\#6) passes above the critical point and expands without experiencing low temperature effects. Figure 3.17 shows a $(T, \rho)$ diagram with evolution of several material zones in a HYADES calculation for $\mathrm{F}=2.5 \mathrm{~J} / \mathrm{cm}^{2}$ laser pulse (same case as in Figure 3.13). The picture shows the type of behavior we just described (in addition, compression by the shock and effects of the initial expansion are visible). 


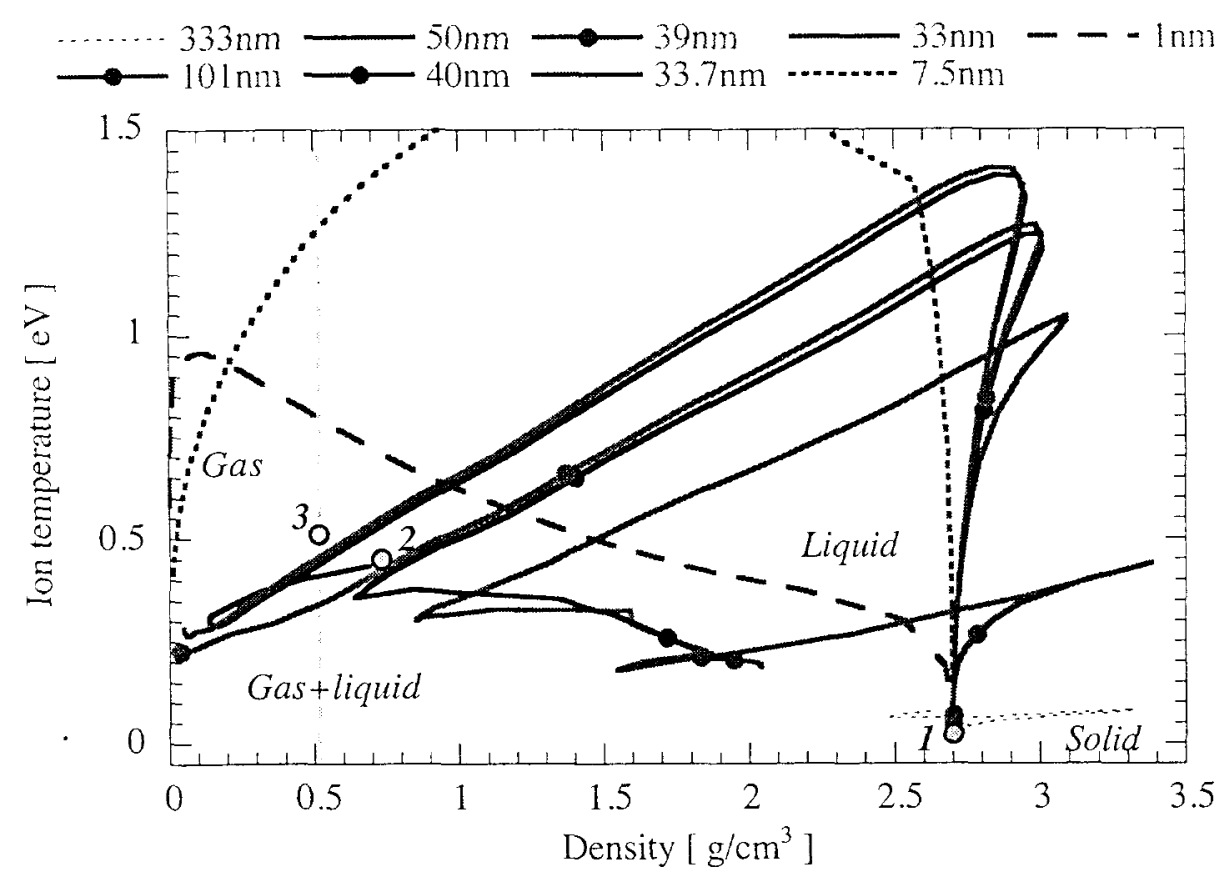

Figure 3.17: $(T, \rho)$ diagram describing evolution of material zones after ablation with $800 \mathrm{~nm}, 100 \mathrm{fs}$, normally incident laser pulse with fluence $2.5 \mathrm{~J} / \mathrm{cm}^{2}$. Material is aluminum. Calculation time is $400 \mathrm{ps}$. The labels show initial coordinate of the zone. Orange dots are 1: normal state $\left(\rho_{0}=2.7 \mathrm{~g} / \mathrm{cm}^{3}, T=300 \mathrm{~K}\right) ; 2$ : a point where negative pressure appears outside of the unstable region $\left(\rho_{0}=0.73 \mathrm{~g} / \mathrm{cm}^{3}, T=0.45 \mathrm{eV}\right.$ [Sesame83]); 3 : critical point $\left(\rho_{0}=0.51 \mathrm{~g} / \mathrm{cm}^{3}, T=0.5 \mathrm{eV}[\right.$ Sesame 83$\left.]\right)$.

Especially interesting is the evolution of the two pairs of zones that correspond to the initial coordinates of approximately $33 \mathrm{~nm}$ and $40 \mathrm{~nm}$. These are adjacent zones, however, when they get into the gas-liquid region, they experience very different fates. One continues expansion as a gas, the other becomes liquid. On the other hand, material zones that pass above the critical point smoothly transform from liquid to gas without sensing the effects of the material strength. Such behavior shows that the liquid-vapor critical 
point can be used for the indication of the amount of material removed [Zel'dovich67, Vidal01]. In particular, we suggest to estimate the depth of the material removed as the initial coordinate of the deepest zone that expands as a gas. This zone has a final temperature and density below critical. The material that has a smaller initial depth becomes an ablation plume and is considered to be removed. Even if there is some condensation in the plume, the liquid droplets have no way to stay with the bulk. On the other hand, the deeper part of the material remains liquid and has no way to become gas, because it is already cold and experiences effects of the material strength. For the calculations represented by Figure 3.17 the material removal is estimated as $39 \mathrm{~nm}$. This is the same number we obtained using the zero velocity zone criterion. Out of these $39 \mathrm{~nm}$, a $35 \mathrm{~nm}$ layer is removed as a pure gas (it has no liquid droplets) and the rest is a gas-liquid mixture.

We have to say that this criterion serves only as an estimate. Gas bubble formation is essentially a three-dimensional process - our simulations are onedimensional. The hydrocode deals with matter in thermodynamic equilibrium and doesn't know anything about phase dynamics - the best we can do is to use an equation of state that doesn't have the Maxwell's construction. We do not account for the possible effects of hydrodynamic instabilities on the gas-liquid boundary (the reason for melt splashing). Effects of material evaporation from the surface of the liquid are also omitted. However, 
USLP ablation creates a relatively thin melted layer (when compared to the long pulse ablation) that cools off quickly, thus we can hope that these effects are not dominant.

Since it is crucial to have a quantitatively correct description of the liquid-vapor region, we have to point out that a popular quotidian equation of state [More88] very often gives an incorrect prediction of the critical point (for example for aluminum it gives $T_{c} \sim 1.5 \mathrm{eV}$ versus experimental data $T_{c}=0.5 \mathrm{eV}$ ) or the liquid-vapor region is absent completely. One has to use the Sesame EOS [Sesame83] (as we did for aluminum) or use the modified quotidian EOS [Young95].

To demonstrate this criterion, we apply it to the calculations of ablation of aluminum with $100 \mathrm{fs}, 800 \mathrm{~nm}$, normally incident USLPs - the same calculations we used to study absorption (section 2.7) and pressure waves (section 3.4). After the pulse is absorbed, the calculations were continued for time up to a nanosecond until the plume cools off enough for the material to reach the gas-liquid region. Once the material passes through the unstable zone, we can calculate the depth of the material removed as gas and total removal depth in the form of gas and liquid. For completeness we also calculated the depth of the melted layer at the end of the run. Although the spreading of this layer has not stopped yet, it is significantly slowed down, since the thermal diffusion length is proportional to $\sqrt{t}$. Figure 3.18 shows a plot of these parameters, together with absorption, versus the absorbed fluence. The calculations show that the removal appears for laser fluences above $0.6 \mathrm{~J} / \mathrm{cm}^{2}$ (shaded region on the left shows no removal zone). At a 
$3.84 \mathrm{~g} / \mathrm{cm}^{3}\left(\rho / \rho_{l}=1.4\right)$, Hugoniot intersects an isentrope that crosses equilibrium curve at the point
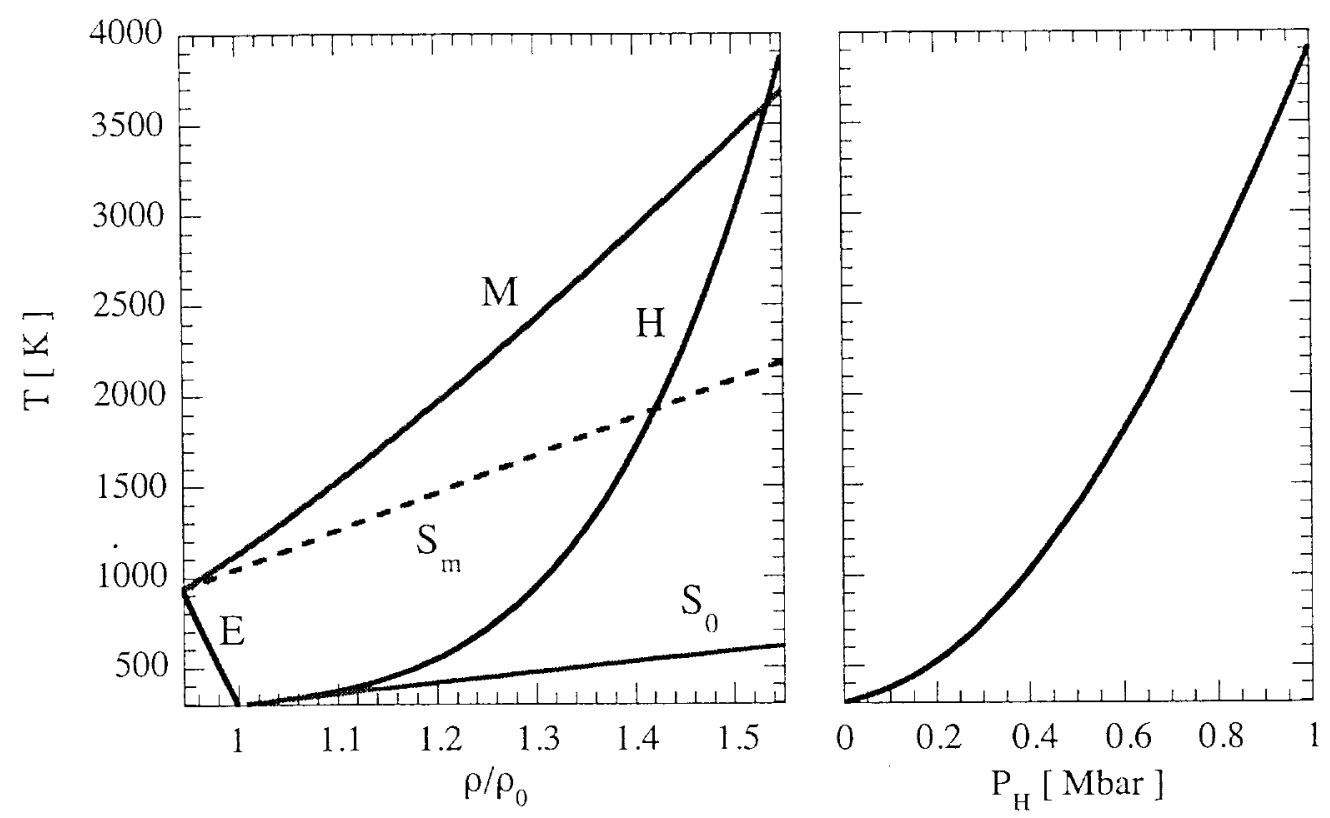

Figure 3.5: Left plot: $(T, \rho)$ diagram with Hugoniot (H, blue), melting (M, red), equilibrium at atmospheric pressure (E, black), and isentrope $\left(S_{0}\right.$ and $S_{m}$, green) curves for aluminum. Right plot: Temperature along the Hugoniot of aluminum as a function of shock pressure.

where melting begins. It corresponds to the shock pressure of $623 \mathrm{kbar}$. This number is close to $608 \mathrm{kbar}$ obtained in calculations with different equation of state [Henis93]. Since the melting curve stays above the Hugoniot and isentrope, the material remains solid during the whole cycle of compression and expansion. In order to melt aluminum directly, the shock pressure must be above $931 \mathrm{kbar}$. The melting occurs at $4.14 \mathrm{~g} / \mathrm{cm}^{3}$ density and $3600 \mathrm{~K}$ temperature. It was calculated [Henin93] that complete melting of 
aluminum on release occurs for shock pressure above $790 \mathrm{kbar}$.

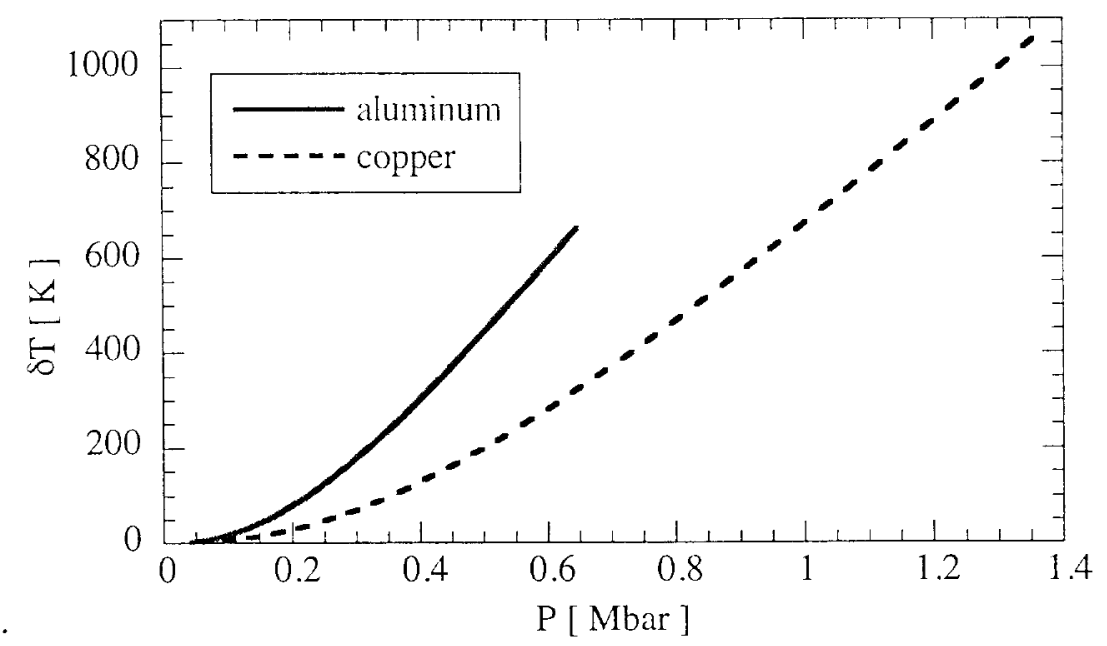

Figure 3.6: Residual temperature increase in aluminum and copper as a function of shock pressure.

Figure 3.6 shows the residual temperature of aluminum and copper as a function of shock pressure. Up to pressures of $100 \mathrm{kbar}(\mathrm{AI})$ and $200 \mathrm{Kbar}(\mathrm{Cu})$ there is practically no heating: the Hugoniot is approximately an isentrope. Melting of copper on shock relaxation begins when the shock pressure is $1.36 \mathrm{Mbar}$. This corresponds to the compressed density $12.73 \mathrm{~g} / \mathrm{cm}^{3}\left(\rho / \rho_{\eta} \sim 1.43\right)$. Like aluminum, copper stays solid during the whole cycle (its $(T, \rho)$ diagram is qualitatively the same as the one for aluminum in Figure 3.5). Melting of copper on compression occurs at a density of $13.66 \mathrm{~g} / \mathrm{cm}^{3}$ and temperature $5727 \mathrm{~K}$. It corresponds to the shock pressure $2.08 \mathrm{Mbar}$.

Reaching pressure in the Mbar range with conventional means, like explosives or 
short pulse lasers, is difficult. However, we saw in the previous section that USLP ablation with fluences of only a few $\mathrm{J} / \mathrm{cm}^{2}$ generates Mbar pressures. Figure 2.23 shows that tens and hundreds of Mbars of pressure are within reach. This pressure does drop with shock propagation into the bulk of the material (and quite significantly as Figure 3.2 shows). However, for moderate and high energy USLPs, the shock waves are an energy transport mechanism competing with thermal diffusion in the near surface zone. In fact, due to the high velocity of propagation, they may dominate energy deposition in the bulk.

\subsection{Evolution of USLP generated shock waves}

Laser induced pressure pulses can be interesting for a variety of applications. In this section we study behavior of the pressure pulses as a function of propagation distance and laser fluence. First, let us describe material response in the two extreme cases of laser-material interaction: ablation with small and large pulse energy. The meaning of small and large is explained further in the text.

We consider two simulations of ablation of aluminum by normally incident laser pulses that have $100 \mathrm{fs}$ pulse width and $800 \mathrm{~nm}$ wavelength. The low energy pulse intensity is $10^{12} \mathrm{~W} / \mathrm{cm}^{2}$ and fluence is $0.106 \mathrm{~J} / \mathrm{cm}^{2}$. Absorption of this pulse is described in detail in section 2.7 and evolution of the major material parameters during the absorption is presented in Figure 2.18. The figure shows that at the end of the laser pulse a surface layer approximately $50 \mathrm{~nm}$ thick (width of the electron temperature profile) is heated. 

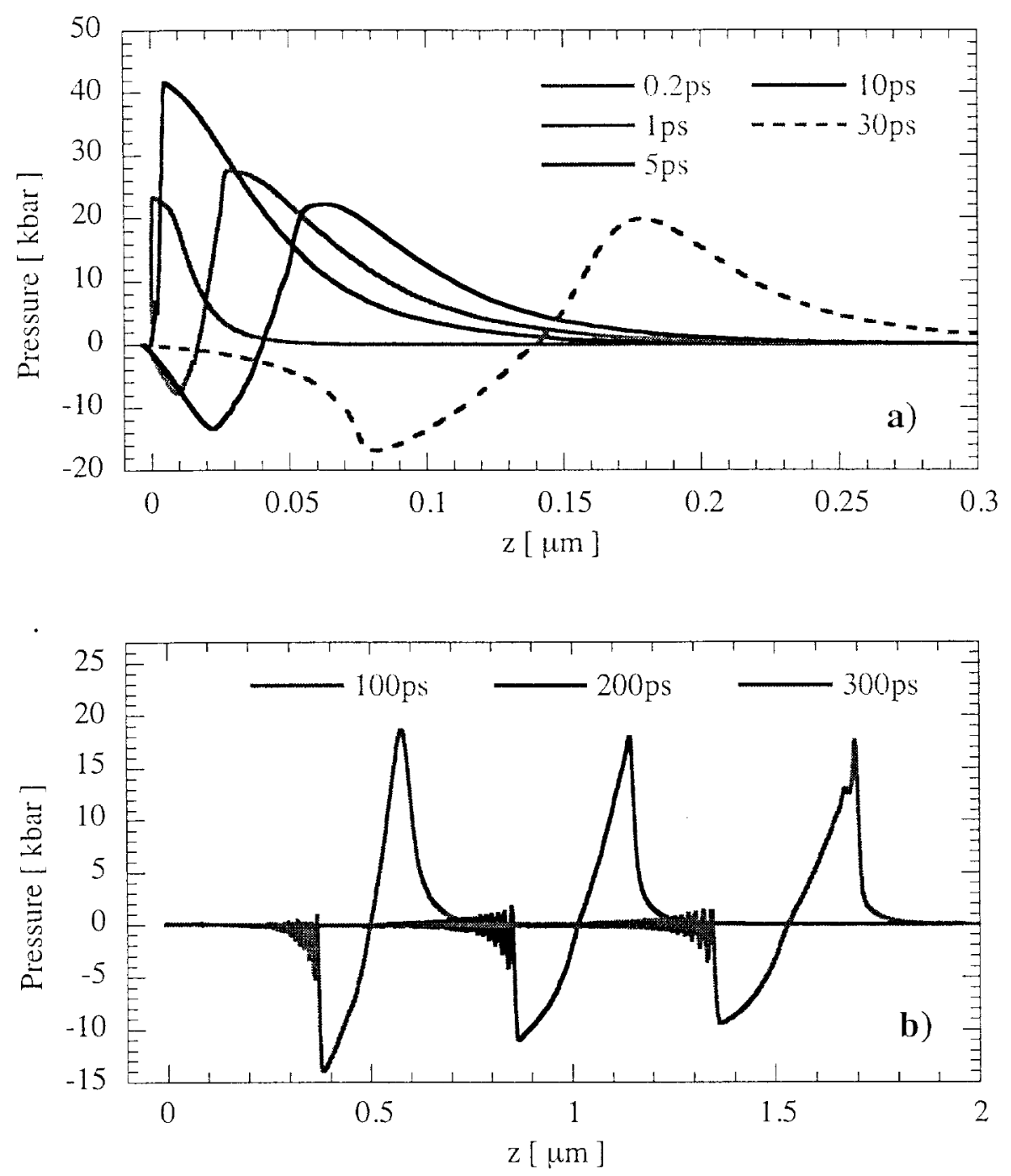

Figure 3.7: Evolution of USLP generated pressure in aluminum. Laser pulse parameters are $800 \mathrm{~nm}$ wavelength, $100 \mathrm{fs}$ pulsewidth, $0.1 \mathrm{~J} / \mathrm{cm}^{2}$ fluence. Laser is incident from the left and the material originally occupies region $z>0$. Oscillations in the tail of the pulse are numerical noise.

The temperature increase raises material pressure and launches a pressure wave propagating into the material. Assuming sound speed does not change significantly, the 
pressure pulse forms and leaves the heated zone in a few tens of picoseconds. Figure $3.7 \mathrm{a}$ describes this initial stage. We can notice that the pressure peaks after the pulse is over. From $0.2 \mathrm{ps}$ to $1 \mathrm{ps}$ it has the same exponential-like profile, but increases in width and amplitude. During the absorption most of the deposited energy is stored in the electrons, which do not create significant pressure in this range of temperatures. As they transfer energy to the lattice, the pressure profile develops. After 1ps, effects of the free surface and material expansion become visible. Since the external pressure is zero and the material is free to move, increase in the thermal pressure $P_{T}$ causes the material to expand. However, the cold pressure $P_{c}$, created by the interparticle interaction, is negative in the expansion (tensile stress) and counteracts effects of the thermal pressure. The maximum tensile stress, which a material is capable of developing, is called a tensile strength. If the thermal pressure is very high, it can overcome particle attraction, which is significant only within relatively small variations of volume, and the material expands as if it were a gas. Otherwise, the expansion stops at some level and the material contracts back to an equilibrium volume, albeit a different one from the original, because the material is still heated. The maximum thermal pressure the material is able to withstand can be estimated using the cohesive energy and the bulk modulus. The cohesive energy $U_{c}$ is the energy required to form separated neutral atoms from the solid at zero temperature and its typical value is approximately $300 \mathrm{~kJ} / \mathrm{mol}$ [Kittel96]. The bulk modulus $B$ is a coefficient relating compressive pressure to the relative volume change 


$$
\frac{\delta V}{V}=-\frac{P}{B}
$$

It is on the order of $10^{12} \mathrm{dyne} / \mathrm{cm}^{2}=1 \mathrm{Mbar}$ [Gray63]. Energy required for the expansion is $P \delta V$. Setting this energy equal to the cohesive energy and expressing $\delta V$ from the formula for the bulk modulus, we arrive at an expression for the maximum tensile stress the material can develop

$$
P=\sqrt{U_{c} B \rho}
$$

Grady [Grady88] gives a formula that is different only by a numerical coefficient

$$
P=\sqrt{U_{c} B \rho / 8} \text {. }
$$

The product $\rho U_{c}$ has a similar value for many materials: $30 \mathrm{~kJ} / \mathrm{cm}^{3}=0.3 \mathrm{Mbar}$ [Gray63]. Thus, the maximum tensile stress is approximately $200 \mathrm{kbar}$. In our case the thermal pressure is noticeably smaller, so the expansion of aluminum is limited.

In order to stop the material movement, negative pressure appears. When the expansion is stopped and material contraction begins, a negative pressure pulse forms and follows the positive pressure pulse in the propagation into the bulk of the material. This pressure waveform is called a bipolar pressure wave. In our case the amplitude of the pressure variation is $20 \mathrm{kbar}$. Propagation of this wave at a longer time is shown in Figure 3.7b. The pressure pulse has already left the heated surface zone, which had its temperature go above the melting temperature only briefly for a few ps after the absorption. During the propagation, there is gradual steepening and shock formation. 
Since the variation in sound speed is small in this range of pressures, this process is quite slow: 200ps after the absorption the shock at the front of the pulse is not yet formed. The amplitude of the wave slowly decreases in addition to some spreading of the pulse (at $300 \mathrm{ps}$, full width is approximately $400 \mathrm{~nm}$ - up from an initial $200 \mathrm{~nm}$ ). Bipolar pulses are also observed in the long pulse (ns) laser absorption, however, as in the case of regular pressure waves, their width is determined by the width of the laser pulse. In the USLP case it is determined by the initial width of the pressure profile.

If the laser-generated pressure is weaker than the material strength, this kind of pressure wave formation and propagation is typical. This is what we meant by small laser energy absorption. Next, lets review a situation when the complete opposite is true - a case when the thermal pressure is much larger than the cold pressure: ablation with a high fluence pulse. Laser fluence was set to $700 \mathrm{~J} / \mathrm{cm}^{2}$. The absorption was $23.15 \%$. In this case, most of the interaction occurs with the plasma and the electron and ion temperatures at the pulse peak reach $275 \mathrm{eV}$ and $24 \mathrm{eV}$ respectively. The pressure at the same moment peaks at $160 \mathrm{Mbar}$. The formation and initial evolution of the pressure pulse is shown in Figure 3.8a. The pressure pulse propagates very fast and by $5 \mathrm{ps}$ it leaves the surface heated zone and the shock wave is already formed. The pulse has a triangular-like form and it noticeably widens and drops in amplitude along the propagation path (see Figure $3.8 b)$. 

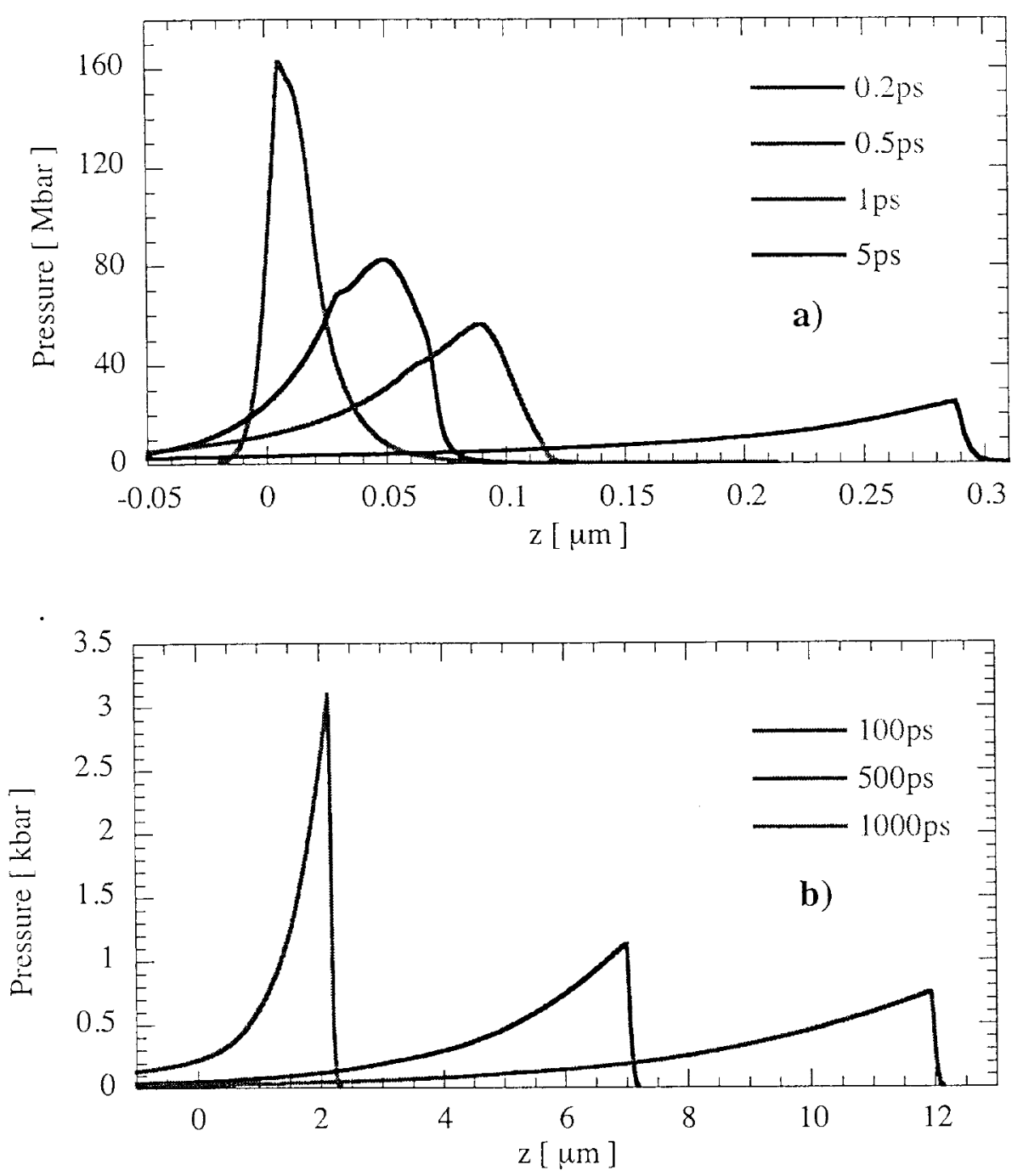

Figure 3.8: Evolution of USLP generated pressure in aluminum. Laser pulse parameters are $800 \mathrm{~nm}$ wavelength, $100 \mathrm{fs}$ pulsewidth, $700 \mathrm{~J} / \mathrm{cm}^{2}$ fluence.

However, even 1 ns after the absorption, the peak pressure is $800 \mathrm{kbar}$ (enough to completely melt aluminum on the expansion). The peak is located at $12 \mu \mathrm{m}$ from the original surface. This corresponds to an average supersonic propagation velocity of $12 \mathrm{~km} / \mathrm{s}$. According to the formula (2.26) the thermal diffusion length after $1 \mathrm{~ns}$ is 
approximately $1 \mu \mathrm{m}$. This is a regime where energy transport into the bulk is dominated by the shock.

In order to understand the evolution of the pulse, we plotted peak pressure of the shock wave, its location, and the width of the pulse (defined as the full width at half maximum) versus time (see Figure 3.9).

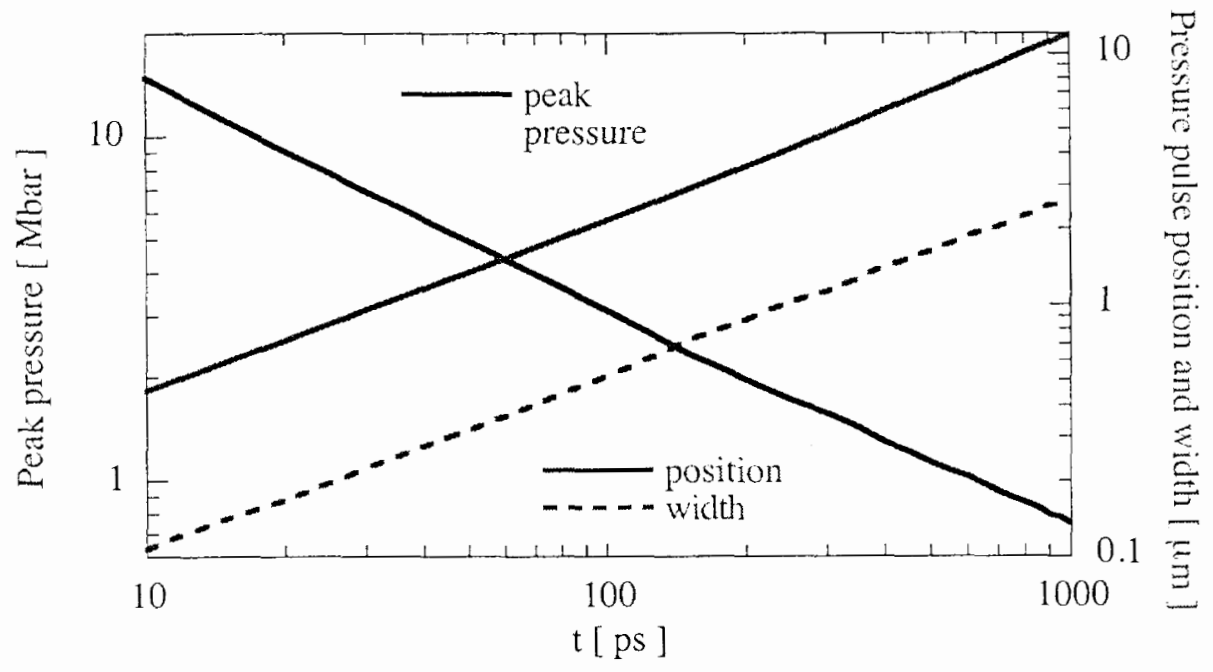

Figure 3.9: Evolution of the parameters of the USLP generated shock wave in aluminum.

Laser pulse parameters are $800 \mathrm{~nm}$ wavelength, $100 \mathrm{fs}$ pulsewidth, $700 \mathrm{~J} / \mathrm{cm}^{2}$ fluence.

On the log-log plot the curves look like straight lines. This means the parameters have power law $t^{\alpha}$ time dependence. In addition to this, the width of the shock and its position have a similar dependence on time. Fitting the data reveals that there are actually two regions. For the multi Mbar regime (from 10ps to 100ps) the following fit was obtained 


$$
\begin{gathered}
P_{p}=14.63 \mathrm{Mbar}\left(\frac{t}{10 \mathrm{ps}}\right)^{-0.678} \quad z_{p}=0.45 \mu \mathrm{m}\left(\frac{t}{10 \mathrm{ps}}\right)^{0.674} \\
\mathrm{w}=0.106 \mu \mathrm{m}\left(\frac{t}{10 \mathrm{ps}}\right)^{0.685}
\end{gathered}
$$

In the formulas the time is in picoseconds. For the time after $100 \mathrm{ps}$, when the pressure is on the order of Mbar or lower, the fit gives

$$
\begin{gathered}
P_{p}=2.96 \mathrm{Mbar}\left(\frac{t}{100 \mathrm{ps}}\right)^{-0.595} \quad z_{p}=2.08 \mu \mathrm{m}\left(\frac{t}{100 \mathrm{ps}}\right)^{0.757} . \\
\mathrm{w}=0.536 \mu \mathrm{m}\left(\frac{t}{100 \mathrm{ps}}\right)^{0.687}
\end{gathered}
$$

The high pressure region is especially interesting. Except for the negative sign in the pressure fit, powers $\alpha$ are the same for pressure, distance and width and equal to approximately 0.68 . In this case the pressure is proportional to the inverse of the shock position: $P_{p} \sim 1 / \tau_{p}$. Once the pressure drops to a Mbar range, shock propagation accelerates and the decrease in the pressure slows down. Power law dependence on time is still a very good fit, but the powers are somewhat different.

The behavior of the USLP generated shock in the multi Mbar regime is universal. For different pulses (as long the amount of the deposited energy is significant), different materials (for example copper) the shock parameters as a function of time are described by the power law with the same power $\alpha \sim 0.66-0.68$.

This kind of pressure evolution can be explained using a self-similar solution of hydrodynamic motion of an ideal gas. In the previous section we showed that the material 
pressure and energy can be split in cold and thermal parts. The cold part, dominant at low temperatures, is a function of volume only. On the other hand, the contribution of thermal effects grows with temperature and at some point becomes much larger than the potential interaction. In this case, the material response is that of an ideal gas. In fact, in some cases, material behaves as an ideal gas even at low temperature. If the Grüneisen gamma $\Gamma$ can be modeled as a constant function of volume, adiabatic compression is described by the solution of the equation (3.17)

$$
T(V) / T\left(V_{0}\right)=\left(V_{0} / V\right)^{\Gamma}
$$

This is a formula for isentropic compression of an ideal gas with isentropic exponent $\gamma=\Gamma+1$ [Landau69]. Therefore, hydrodynamic solutions obtained for an ideal gas are relevant to our problem.

Hydrodynamic motion of an ideal gas in the planar case is described by the following equations [Zel'dovich67]

$$
\begin{aligned}
& \frac{1}{\rho} \frac{\partial \rho}{\partial t}+\frac{u}{\rho} \frac{\partial \rho}{\partial z}+\frac{\partial u}{\partial z}=0 \\
& \frac{\partial u}{\partial t}+u \frac{\partial u}{\partial z}+\frac{1}{\rho} \frac{\partial P}{\partial z}=0 \\
& \frac{\partial}{\partial t} \ln \left(P \rho^{-\gamma}\right)+u \frac{\partial}{\partial z} \ln \left(P \rho^{-\gamma}\right)=0
\end{aligned},
$$

where thermal transport (thermal diffusion, etc.) is omitted for simplicity. Suppose one found a solution for a particular problem 


$$
P=f_{1}(z, t), \rho=f_{2}(z, t), u=f_{3}(z, t)
$$

The equations (3.36) admit several similarity transformations that allow to get a different solution from a known one, in case initial and boundary conditions are also properly transformed [Zel'dovich67,Sedov93]. For example, multiplying density and pressure by a factor $k$ and leaving the remaining variables unchanged does not change the equations (3.36). Therefore functions

$$
P=k f_{1}(z, t), \rho=k f_{2}(z, t), u=f_{3}(z, t) .
$$

are also solution of the hydrodynamic equations. Other possible similarity transformations are scaling of time and length. Applying these transformations in succession one can obtain an infinite number of solutions. It is important to note that the equation of state must also allow these transformations (this is the case for an ideal gas).

The hydrodynamic equations also admit a type of motion, which is similar to itself. The self-similar solutions describe a motion such that only scales of the parameters depend on time, but the profiles (shape and form) remain the same. It often represents asymptotic behavior of solutions that are not self-similar at the beginning. Self-similar solution can be represented as [Zel'dovich67,Sedov93]

$$
P=\rho_{0}(t)\left(\frac{d Z(t)}{d t}\right)^{2} f_{1}(\xi), \quad \rho=\rho_{0}(t) f_{2}(\xi), \quad u=\frac{d Z(t)}{d t} f_{3}(\xi), \quad \xi=\frac{z}{Z(t)},
$$

where $Z(t)$ is the length scale and $\rho_{t}(t)$ is the density scale. Functions $f$ describe spatial profiles of the parameters. Substituting (3.37) in (3.36) we obtain 


$$
\begin{aligned}
& \frac{1}{\rho_{0}} \frac{d \rho_{0}}{d t}+\frac{1}{Z} \frac{d Z}{d t}\left[f_{1}^{\prime}+\left(f_{3}-\xi\right)\left(\ln f_{2}\right)^{\prime}\right]=0 \\
& \frac{Z}{(d Z / d t)^{2}} \frac{d^{2} Z}{d t^{2}} f_{3}+\left(f_{3}-\xi\right) f_{3}^{\prime}+\frac{f_{1}^{\prime}}{f_{2}}=0 \\
& \frac{1}{Z} \frac{d Z}{d t} \frac{d}{d t} \ln \left(\rho_{0}^{1-\gamma}\left(\frac{d Z}{d t}\right)^{2}\right)+\left(f_{3}-\xi\right)\left(\ln \left(f_{1} f_{2}^{-\gamma}\right)\right)^{\prime}=0
\end{aligned}
$$

where $f^{\prime}(\xi)$ describes derivative with respect to variable $\xi$. Self-similar solutions are possible if there is a separation of variables. In order for this to happen, for example in the second equation, the following condition must be satisfied

$$
\frac{Z}{(d Z / d t)^{2}} \frac{d^{2} Z}{d t^{2}}=\text { const }
$$

If the constant is different from one, the solution of this equation is

$$
Z(t)=A t^{\alpha},
$$

where $A$ and $\alpha$ are constants. A similar condition for the function $\rho_{0}$ yields

$$
\rho_{0}(t)=B t^{\beta}
$$

where $B$ and $\beta$ are also constants. Thus, there are self-similar solutions with parameters changing as a power function of time.

In order to verify that the behavior of the shock observed in the high energy USLP ablation is self-similar, we need to look at the evolution of the peak position and width of the shock. The self-similar pressure is

$$
P=B A^{2} \alpha^{2} t^{\beta+2(\alpha-1)} f_{1}\left(\frac{z}{A t^{\alpha}}\right)
$$

Since the shape is preserved, we obtain 


$$
P_{p}=B A^{2} \alpha^{2} t^{\beta+2(\alpha-1)} f_{1}\left(\xi_{p}\right), \quad z_{p}=A t^{\alpha} \xi_{p}, \quad \mathrm{w}=A t^{\alpha}\left(\xi_{p}-\xi_{1 / 2}\right) .
$$

Thus, for a solution to be self-similar, the position of the pressure peak and its width must have the same time dependence. This is what we observe from our simulations in the multi Mbar regime. The constant $\alpha$ is approximately $0.66-0.68$ and the constant $\beta$ is zero. When the pressure drops in the Mbar range, shock behavior is no longer self-similar; however power dependence of variables on time is still a good description.

Material response in our problem is analogous to the motion of a gas under the action of an impulsive load. This kind of motion arises when a gas filling a half-space is exposed to a short pressure pulse created by some means, for example a piston push or a detonation of explosive at the surface [Zel'dovich67]. When the evolution time becomes much larger than the pressure pulse length, the behavior of the shock is self-similar. This is the case in USLP ablation. Analysis of the self-similar solution shows [Zel'dovich67] that the similarity exponent $\beta$ is zero and similarity exponent $\alpha$ must be in a very narrow range

$$
\frac{1}{2}<\alpha<\frac{2}{3}
$$

In the high power USLP ablation exponent $\alpha$ is $2 / 3$.

In moderate fluence ablation both power law dependence and effects of the material strength are observed. Figure 3.10 shows a plot of pressure versus distance at different times after ablation of aluminum by a laser pulse with fluence $2.5 \mathrm{~J} / \mathrm{cm}^{2}$ (all 
other parameters are the same as in low and high energy ablation). The absorption is $19 \%$ and the peak electron and ion temperatures are $7 \mathrm{eV}$ and $1.7 \mathrm{eV}$ respectively. The pressure reaches a maximum of $1.3 \mathrm{Mbar} 100 \mathrm{fs}$ after the pulse peak. By $10 \mathrm{ps}$ it drops to 500kbar. After that the evolution of the shock wave parameters can be described by the fit

$$
P_{p} \sim 2 \mathrm{Mbar} t^{-0.5}, \quad z_{p}=13 \mathrm{~nm} t^{0.89}, \quad \mathrm{w}=10 \mathrm{~nm} t^{0.57},
$$

where time is picoseconds.

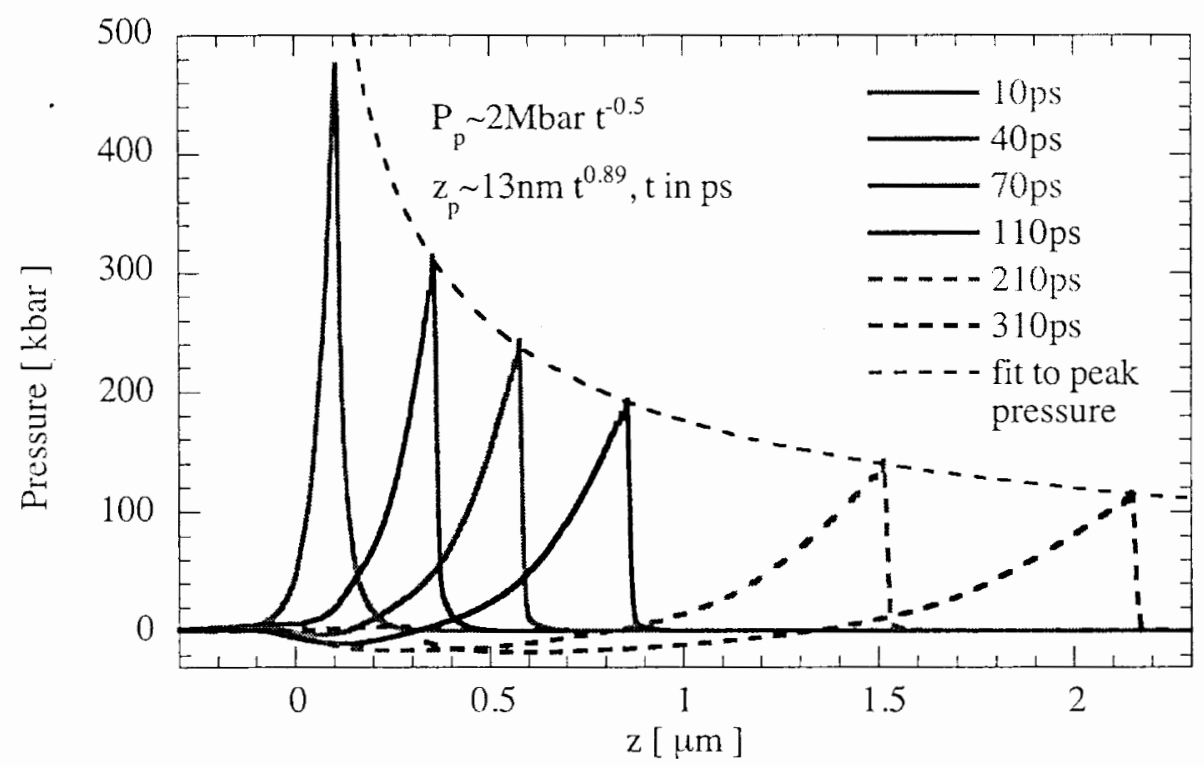

Figure 3.10: Evolution of pressure in aluminum, generated by the laser ablation with $800 \mathrm{~nm}, 100 \mathrm{fs}, 2.5 \mathrm{~J} / \mathrm{cm}^{2}$, normally incident USLP. Black dashed curve shows a power time fit to the shock peak.

Combination of these formulas gives peak pressure dependence on its coordinate as

$$
P_{p} \sim 8 \mathrm{Mbar} z_{p}^{-0.55}
$$


where $z_{i}$ is $\mathrm{nm}$. Pressure pulse propagation speed is approximately $9 \mathrm{~km} / \mathrm{s}$ at $10 \mathrm{ps}$. It decreases with time and eventually approaches the sound speed (longitudinal sound speed in aluminum is $6.4 \mathrm{~km} / \mathrm{s}$ ) and become constant. In this regime, the shock is called a weak shock. The pressure of such a one-dimensional wave has an asymptotic behavior $1 / \sqrt{t}$ [Courant77,Landau59] and the pulse width increases as $\sqrt{t}$. Since the shock speed is practically a constant, pressure decays as $1 / \sqrt{z}$. The damping effect is caused by the nonadiabatic nature of the shock and by the action of the rarefaction wave.

When the shock propagates into the bulk, the material behind is relatively cold and can withstand some tensile stress. Therefore, when the compressed material expands, it goes into the negative pressure region, creating a negative pressure component of the pulse. This part is much wider and weaker than the shock (it is also visible in Figure 3.2). In fact, because of the laser heating, we never see negative pressures that are comparable to the cold material estimate (3.32). Since the total pressure developed in the material is a sum of cold and thermal parts, increase in the thermal pressure lowers the maximum tensile stress the material can withstand.

Figure 3.11 shows evolution of the peak pressure versus time for several cases of ablation of aluminum with $800 \mathrm{~nm}, 100 \mathrm{fs}$ pulses. Laser fluence range from $1.3 \mathrm{~J} / \mathrm{cm}^{2}$ to $1 \mathrm{~kJ} / \mathrm{cm}^{2}$. The maximum pressure appears close to the peak of the laser pulse $(t=0.2 \mathrm{ps})$. Then there is a transition period, when the electron-ion energy exchange occurs and the shock wave forms and leaves the heated zone. The higher the pressure, the faster the 
transition to a power law region. The pressure fit exponents change from -0.5 for moderate energy to $-2 / 3$ for high energy ablation.

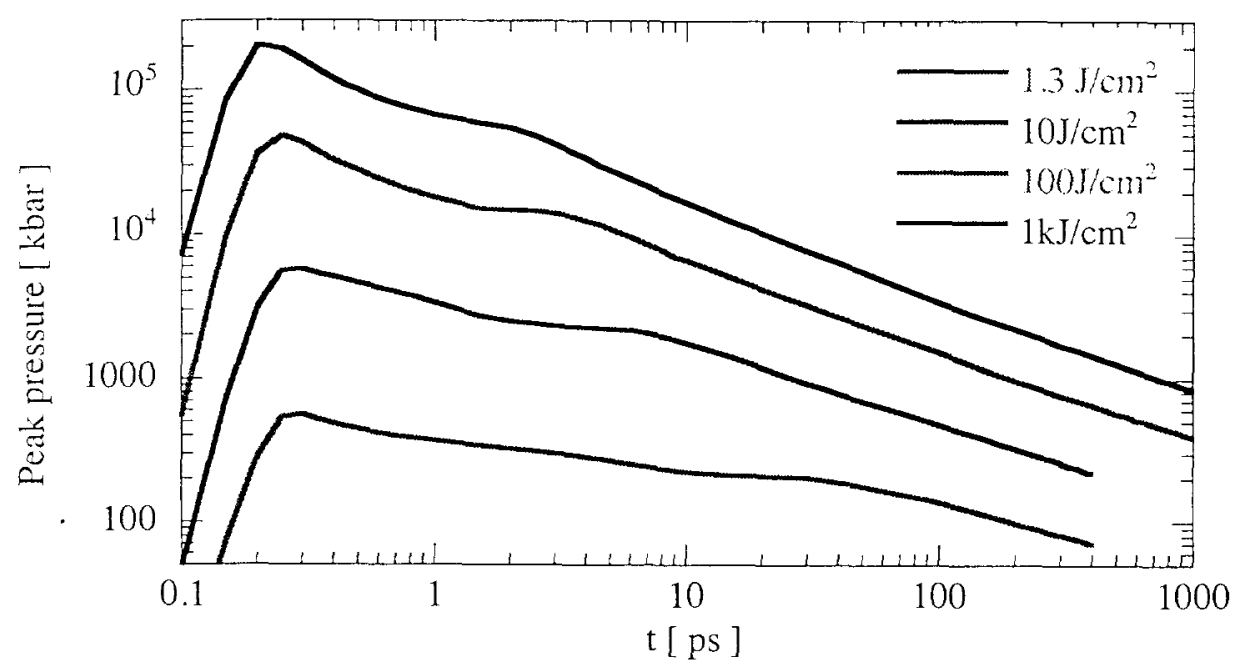

Figure 3.11: Evolution of peak pressure of the pressure wave generated in aluminum by the laser ablation for several different fluences (light wavelength is $800 \mathrm{~nm}$; pulse width is $100 \mathrm{fs}$ and the peak is at $0.2 \mathrm{ps}$; normal incidence).

After examining evolution of the pressure pulses generated by the USLP ablation, the following conclusion can be made. At the low laser fluences, when peak pressure is in the tens of kilobars range and the effects of material strength are significant, the generated pulses have a smooth, double extremum form. This is the so-called bipolar pressure wave. It is essentially a double pulse with positive pressure pulse being followed by a similar in amplitude, but a negative pressure pulse. Since the pressure is relatively small and the sound speed is practically not affected, shock formation is delayed and the pulses can propagate at a constant speed for significant distances and without noticeable 
damping. Increase in the laser fluence results in the generation of higher pressure pulses and accelerated shock wave formation. Simultaneously, higher material temperature lowers material strength, thus the negative pressure part becomes much smaller than the leading, positive pressure pulse. This pulse has a triangle-like form. Despite the fast shock formation and pressure in the hundreds of kilobars range, the shock wave velocity does not deviate significantly from the sound speed (traveled distance $z \sim t$ ). Appearance of the shock means that the motion is not adiabatic and noticeable pressure wave decay appears. Asymptotically the pressure decreases as $1 / \sqrt{t}$ and the pulse width increases as $\sqrt{t}$. The same dependencies expressed in terms of $z$ are $1 / \sqrt{z}$ and $\sqrt{z}$ respectively. Further increase of laser energy creates pulses in the tens and hundreds of Mbar range. At this point material response is similar to the motion of an ideal gas under impulsive load. This type of shock motion is described by a self-similar solution with description of pressure as

$$
P(z, t)=\text { const } t^{2(\alpha-1)} f\left(\frac{z}{A t^{\alpha}}\right)
$$

The self-similarity exponent $\alpha$ can vary from 0.5 to $2 / 3$. We observed it to be close to $2 / 3$. In this case the pressure as a function of the traveled distance decays as $1 / z$ and the pulse width is proportional to $z$. Once the pressure drops into the Mbar and weaker range, the solution is no longer self-similar, but the power-like dependence on time is still a good approximation, albeit with different powers. When the shock speed 
becomes close to the sound speed, the shock returns to the weak shock regime, wave dampening slows down and the pulse behavior is described by the $\sqrt{t}$ time dependence. This description remains valid as long as the propagated distance is much smaller than the transverse laser spot size. When this condition is violated, the decay accelerates due to geometrical, three-dimensional effects.

\subsection{Material ablation and removal}

One of the possible applications of ultrashort lasers is material processing. USLP energy is absorbed in a thin surface layer, which is quickly turned into an energetic, expanding plume. Since the expansion is very fast, energy transport into the bulk is limited. This reduces collateral damage and improves processing quality. Details of the removal depend on the laser and material parameters. Understanding and optimization of the process would greatly benefit if the hydrodynamic simulations could be used to predict the amount of the material removed. In this section we suggest a criterion to estimate the removal and present the results of its application to HYADES calculations in a range of laser parameters.

Material removal is a very complex process that can be accomplished through a variety of mechanisms such as, for example, non-thermal particle ejection, evaporation, ablation (removal through hot vapor and plasma), macroparticle ejection (melt splashing, spallation, etc.) [Bauerle00,Miller98]. Removal can be also counteracted by the particle 
redeposition back on the target or, if, for instance, a deep channel is being drilled, on the walls of the hole. In USLP metal processing, ablation is the dominant mechanism. Removal through melt and evaporation should not be significant, since the bulk energy deposition is expected to be small and, because of the high thermal conductivity, the surface temperature decreases fast after the light beam absorption. In our study we assume a flat surface ablation (no 3D structures), thus redeposition is not an effect (since the plume expands very quickly, redeposition from the USLP ablation plume back on the laser spot should be small).

Intuitively it is obvious that the material transformed into a hot vapor or plasma can be considered removed. It quickly turns into a fast, expanding plume and leaves the bulk of the material. On the other hand, cold, solid-like material doesn't have a high velocity and can not overcome cohesive forces. Thus, it ultimately remains part of the bulk. The high and low laser fluence runs ( $F$ is $0.1 \mathrm{~J} / \mathrm{cm}^{2}$ and $700 \mathrm{~J} / \mathrm{cm}^{2}$ ) described in the previous section represent these two extreme scenarios. Figure 3.12 shows profiles of the aluminum density and temperature $300 \mathrm{ps}$ after the laser absorption. In the low energy case the surface temperature is approximately $600 \mathrm{~K}$ (below the melting temperature) and the surface velocity is practically zero. In fact, the only place where material velocity has noticeable deviation from zero is where the pressure pulse is. Effects of the pressure wave on the density and temperature are quite weak and we can see that the wave is in the bulk, far from the heated surface layer. Thus, there is no material removed. 


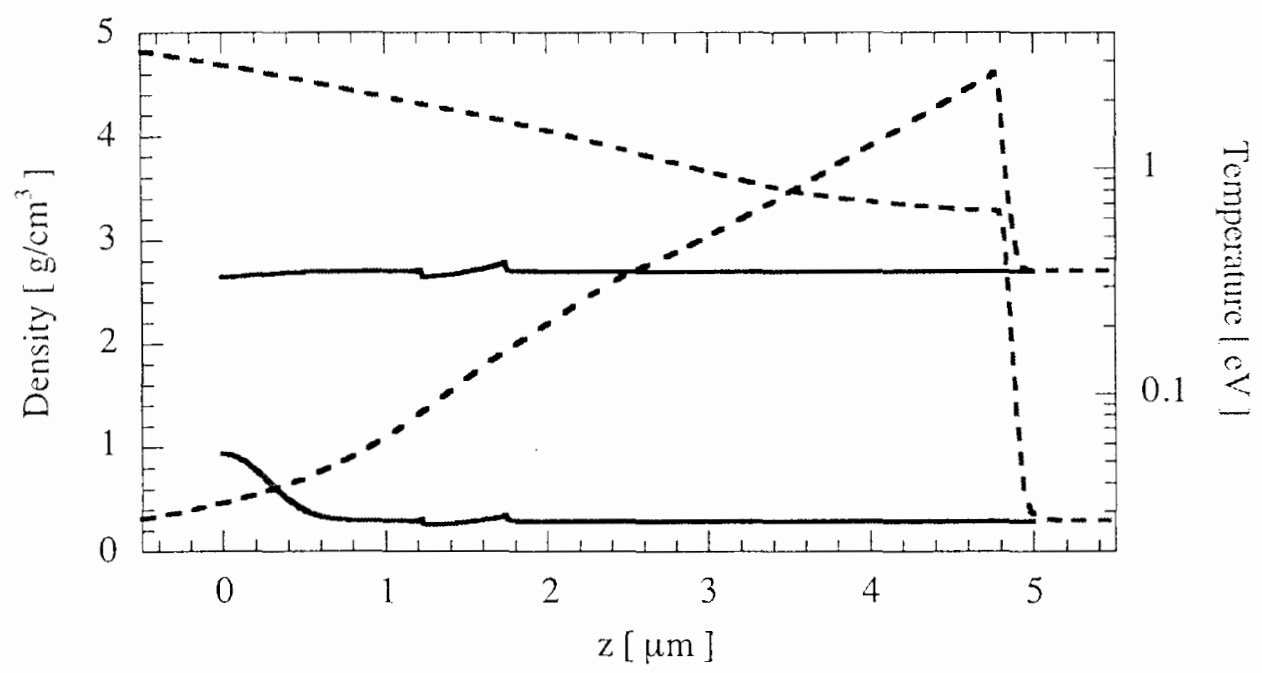

Figure 3.12: Plot of density (blue lines, left axis) and temperature (red lines, right axis) of aluminum versus coordinate $300 \mathrm{ps}$ after the ablation with $800 \mathrm{~nm}, 100 \mathrm{fs}$, normally incident laser pulses. Solid lines correspond to $F=0.1 \mathrm{~J} / \mathrm{cm}^{2}$ and dashed curves are $\mathrm{F}=700 \mathrm{~J} / \mathrm{cm}^{2}$.

We can convince ourselves with a simple estimate. Ratio of the deposited energy flux to the cohesive energy density of the material gives thickness of the removed material layer in the case of maximum efficiency (energy is expended only for overcoming potential attraction). For aluminum the cohesive energy is $U_{c}=327 \mathrm{~kJ} / \mathrm{mol}=32.7 \mathrm{~kJ} / \mathrm{cm}^{3}$ [Kittel 96 ], therefore thickness of this layer is $3.6 \mathrm{~nm}$. This is less than half of the skin depth, where the laser energy is deposited. Thus, energy deposition density is not high enough for the material removal to occur.

We have to notice that this picture is a bit oversimplified. Material removal can occur without expending the energy on the separation of each atom. Variation of laser 
deposition across the beam spot creates uneven thermal expansion and, therefore, mechanical stresses. The heating lowers the material strength, which can be also be weakened by the presence of mechanical defects, contamination, etc. The stresses may become high enough to cause material spallation - breaking off of small pieces of solid material, which is a more energy efficient process than the vaporization of the whole layer. However metals in general are not brittle, the stresses they can withstand are quite high. Thus we do not take this effect into account.

In case of high energy ablation the amount of energy deposited is quite significant. Absorptivity is $23.15 \%$ and $162 \mathrm{~J} / \mathrm{cm}^{2}$ is deposited in the material. Using the same estimate as for the low energy case we get $50 \mu \mathrm{m}$ removal. This number is too large, since even $300 \mathrm{ps}$ after the ablation, the shock reached only 5 um depth and the aluminum is heated to temperature of many $\mathrm{eV}$. Some of the material is certainly removed; the question is how much. One way to estimate this is to do a time integration of mass flux at some fixed plane, for instance $z=0$. For simulations with a Lagrangian hydrocode a more convenient method is to find a zone where the material velocity is zero (zero velocity zone criterion [Komashk099]). Assuming that the material with negative velocity (outward flow) is ablated, initial position of this zone gives the thickness of the removed layer. In this case at $300 \mathrm{ps}$ zero velocity zone is located at $z=1.75 u \mathrm{~m}$. Its initial position is $1.04 \mu \mathrm{m}$. Since material temperature in this zone is quite high (over $1 \mathrm{eV}$ ), the removal 
process hasn't stopped yet. Instead of following the motion of the material for a long time until it cools off, lets review a case when laser has a moderate energy and cooling off happens faster.

Figure 3.13 shows snapshots of material temperature and density versus distance for ablation with laser fluence $2.5 \mathrm{~J} / \mathrm{cm}^{2}$ (this case was reviewed in some details in the previous section). The amount of laser energy deposited is $0.5 \mathrm{~J} / \mathrm{cm}^{2}$.

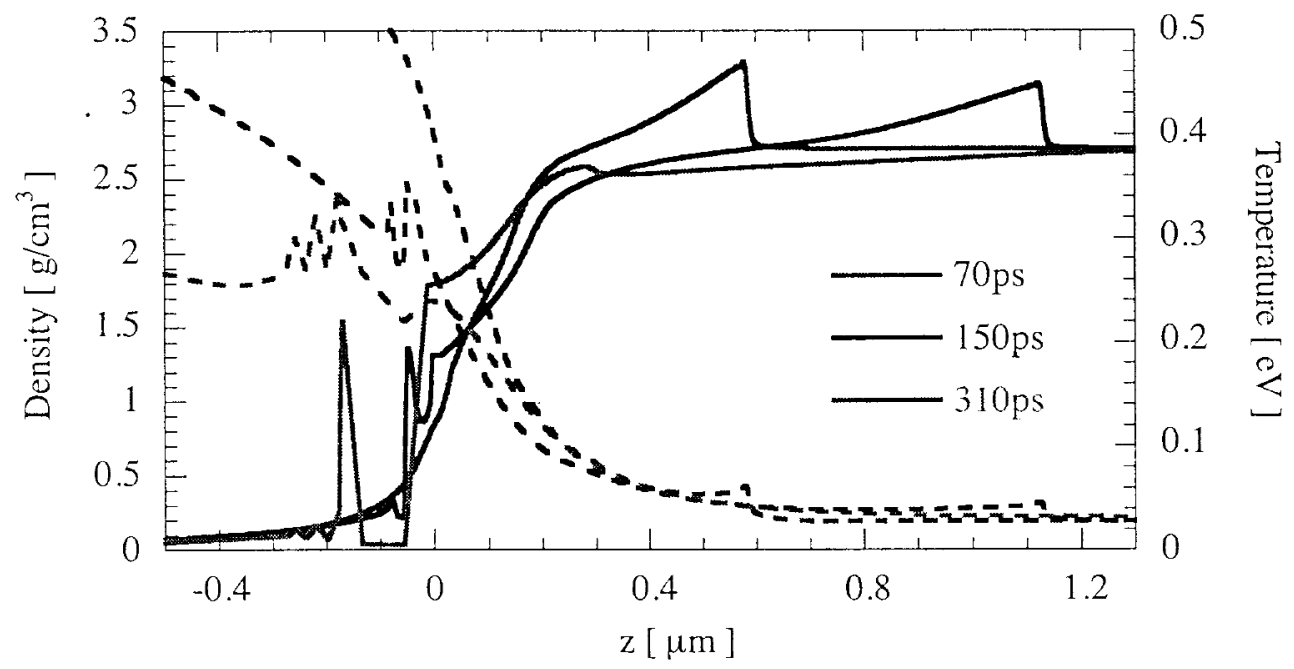

Figure 3.13: Plots of density (solid lines, left axis) and temperature (dashed lines, right axis) of aluminum versus coordinate $70 \mathrm{ps}, 150 \mathrm{fs}$ and $310 \mathrm{ps}$ after the ablation with $800 \mathrm{~nm}, 100 \mathrm{fs}$, normally incident laser pulses with $2.5 \mathrm{~J} / \mathrm{cm}^{2}$ fluence.

The estimate based on cohesive energy gives removal of $153 \mathrm{~nm}$. At $70 \mathrm{ps}$, the zero velocity zone is located at $210 \mathrm{~nm}$ depth and has initial coordinate $180 \mathrm{~nm}$. This is larger than the depth derived from the simple energy estimate, which we thought provides an upper estimate! At later times, the situation is even more complicated. At 150ps and 
similar fluence a melt layer with significant thickness appears (melted material can appear even at lower fluences, however it resolidifies before the end of the run).

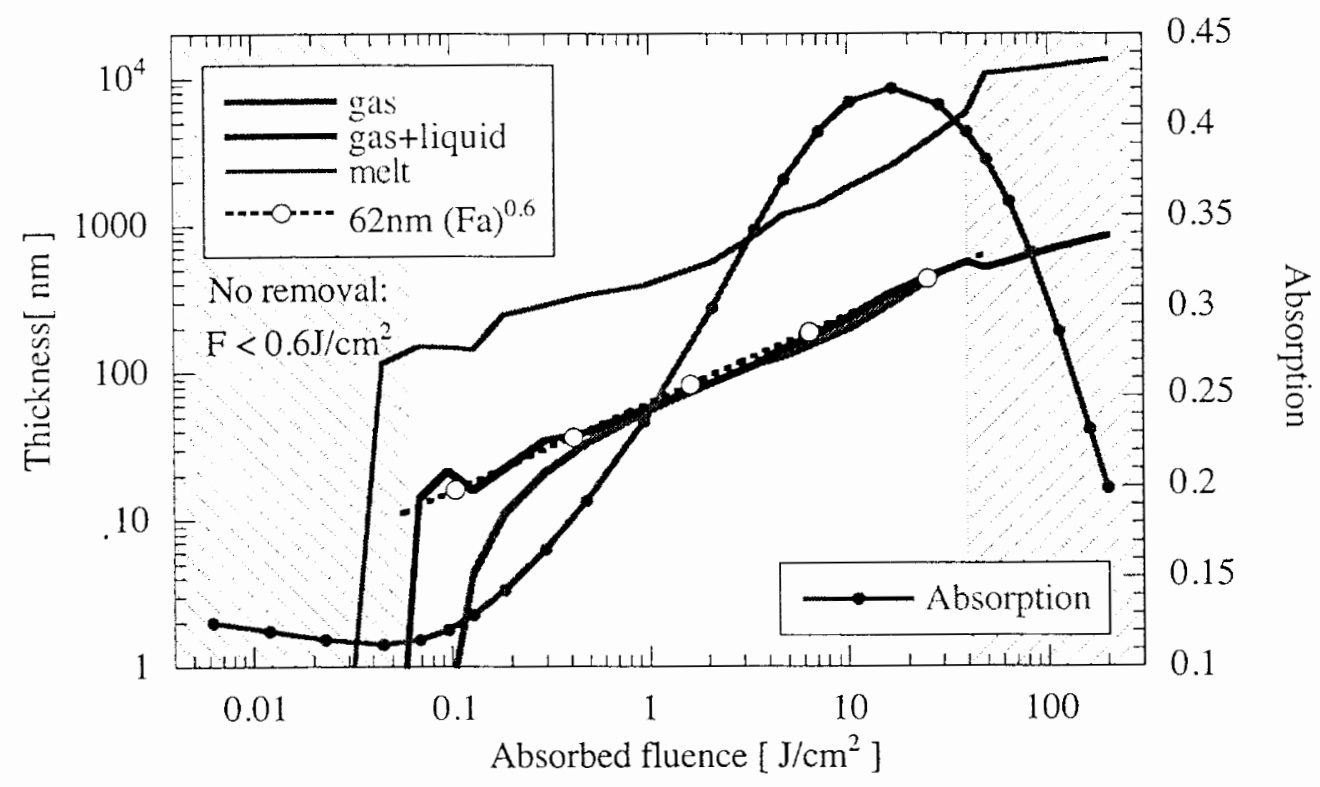

Figure 3.18: Thickness of the removed layer of aluminum as a function of the absorbed laser fluence. Laser parameters - wavelength: $800 \mathrm{~nm}$, pulselength: $100 \mathrm{fs}$, angle of incidence: $0^{\circ}$. The black, dashed curve with circular markers is a power fit to the removed depth (gas and liquid) in the range of absorbed fluence $0.07-40 \mathrm{~J} / \mathrm{cm}^{2}$.

Right above the ablation threshold most of the plume is composed of liquid and gas. With an increase in the laser fluence the fraction of droplets in the plume decreases. For a very high fluence $\left(\mathrm{F}>100 \mathrm{~J} / \mathrm{cm}^{2}\right)$ so much energy is deposited in the material that one nanosecond of the expansion is not enough for the plume to cool off and reach the gasliquid region. In other words the front part of the plume has density below the critical, but temperature is above the critical. This is the shaded region on the right. In the region 
where we have reliable calculation of the removal $\left(\mathrm{F}<100 \mathrm{~J} / \mathrm{cm}^{2}, \mathrm{Fa}<40 \mathrm{~J} / \mathrm{cm}^{2}\right)$ the amount of the removed material (gas+liquid) can be fitted with a power law dependence on the absorbed fluence $\mathrm{Fa}$ (black, dashed line with circle markers)

$$
z_{R}=62 \mathrm{~nm}(F a)^{0.6}=11.5 \mathrm{~nm}\left(\frac{F a}{0.06 \mathrm{~J} / \mathrm{cm}^{2}}\right)^{0.6}
$$

Since the changes in laser energy are orders of magnitude and the absorptivity varies between 0.1 to 0.43 , a power law is also a good approximation for fitting $z_{R}$ versus incident laser fluence

$$
z_{R}=12 \mathrm{~nm}\left(\frac{F}{0.6 \mathrm{~J} / \mathrm{cm}^{2}}\right)^{0.75}
$$

This type of dependence allows us to make an important conclusion about the efficiency of USLP removal. If one calculates the amount of material removed per unit of laser energy (specific removal)

$$
\frac{z_{R}}{F} \sim F^{\alpha}(\alpha<0)
$$

it becomes obvious that the most efficient processing is at or near the threshold. If, for example, the average power of the USLP system is fixed, the removal speed is the fastest when the pulse energy is lowered to the threshold and the repetition rate is increased. Also at the removal threshold the amount of melted material is the smallest. In general, the thickness of the melt layer increases with fluence similarly to the removal depth and stays about ten times larger. 
Since the laser pulses are so short, the material evolution and laser absorption are effectively decoupled, so we presented the removal calculations as a function of the absorbed fluence. If we have a beam incident at an angle, the only effect it has is to change the amount of laser energy absorbed. However for one laser parameter the relationship between absorption and removal is not trivial. If the pulse length is two long, expansion becomes noticeable. This changes the amount and location of the energy deposition. To check the effect of the pulselength, we fixed the laser fluence and calculated the removal for varying pulsewidth. Figure 3.19 shows the result for two cases: $1 \mathrm{~J} / \mathrm{cm}^{2}$ (near threshold removal) and $10 \mathrm{~J} / \mathrm{cm}^{2}$. Pulselength was varied from $50 \mathrm{fs}$ to $50 \mathrm{ps}$. In the low energy case the removal changes weakly between $15 \mathrm{~nm}$ and $25 \mathrm{~nm}$. The most visible transformation is an increase of the plume temperature that results in a smaller fraction of the material removed as a gas-liquid mixture. The needed energy comes from the increase in the absorptivity. Since this is a near threshold case, the plume expansion is slow and has no time to create the plasma shielding effect. This is, probably, why there is no significant effect of longer pulselength on the removal. On the other hand, in the $10 \mathrm{~J} / \mathrm{cm}^{2}$ case, there is a clear transition between subpicosecond and multipicosecond regimes. For pulselength below one picosecond, the removal is practically constant at $110 \mathrm{~nm}$. With increase of pulse duration comes a noticeable increase in the absorption, however the removal drops down to almost $50 \mathrm{~nm}$. The extra 

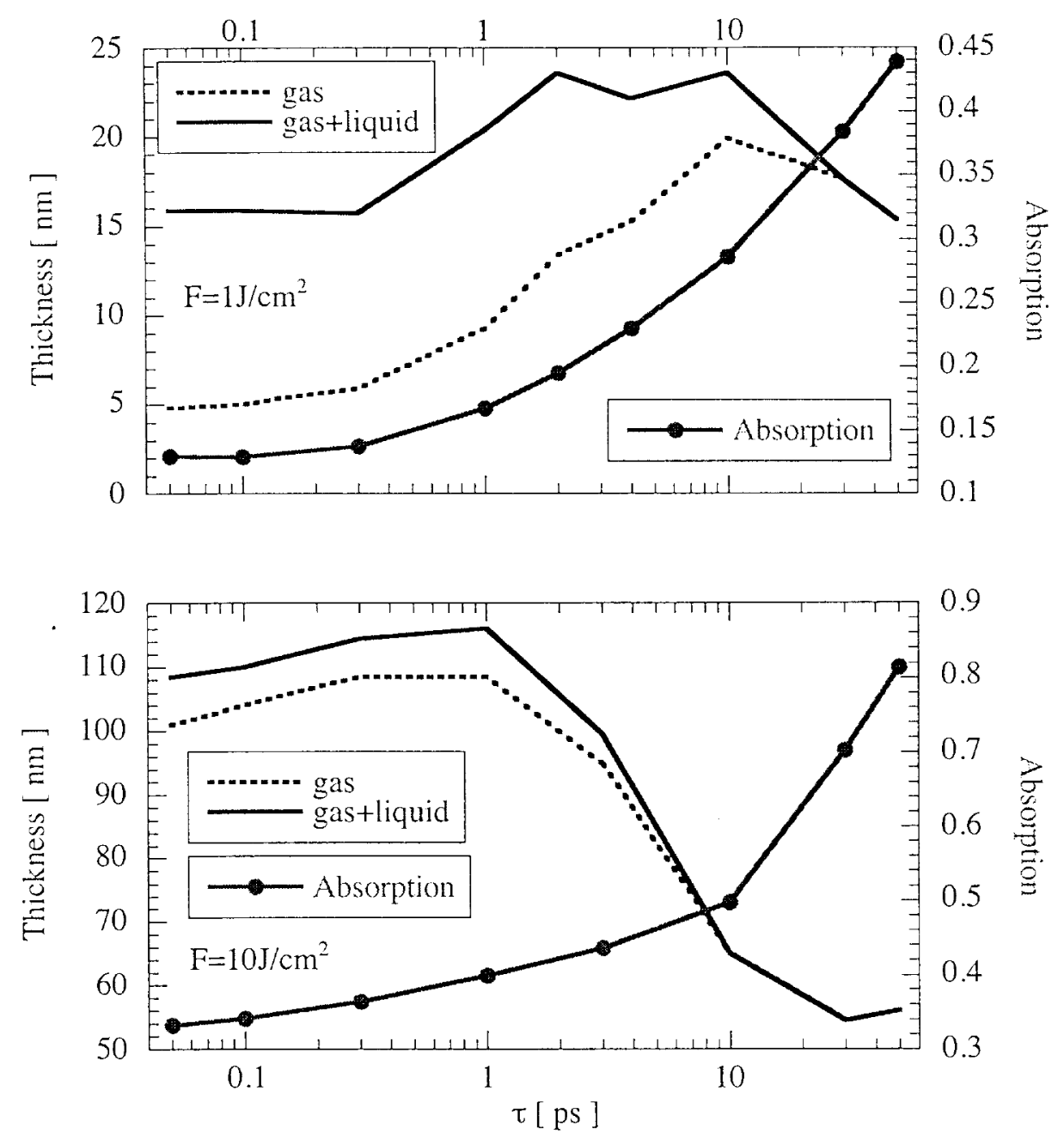

Figure 3.19: Thickness of the removed aluminum layer as a function of laser pulselength. Laser wavelength is $800 \mathrm{~nm}$ and normal incidence. Top picture: laser fluence is $1 \mathrm{~J} / \mathrm{cm}^{2}$; bottom picture: laser fluence is $10 \mathrm{~J} / \mathrm{cm}^{2}$.

deposited energy is absorbed in the expanding plume and does not reach the bulk. We should note that the thickness of the melted layer in both of these cases changes in a similar way to the removal depth.

This is the type of behavior we would expect in ablation of flat surfaces. 
However, very often USLPs are used to create three-dimensional structures, such as high aspect ratio holes. In this case one has to consider removal efficiency together with the absorption. To remove material at the bottom, the pulse must propagate through the partially finished hole. Some of the energy is absorbed in the walls. Since increasing the pulse length tends to increase the absorption, it is preferred to work with shorter pulses, even if in the flat ablation case the same removal is obtained.

We carried out similar calculations (same laser parameters as in Figure 3.19) for copper: In these calculations the modified quotidian EOS [Young95] was used. The general behavior (Figure 3.20) is similar to that of aluminum with a few exceptions. Material removal is somewhat smaller and exhibits a slower growth with fluence

$$
z_{R}=58 \mathrm{~nm}(F a)^{0.55}
$$

The probable reason for this difference is the higher thermal conductivity of copper (3.9W/cmK versus $2.37 \mathrm{~W} / \mathrm{cmK}$ for aluminum); it increases diffusion of the deposited energy in the bulk. Another difference is the much higher ablation threshold approximately $5 \mathrm{~J} / \mathrm{cm}^{2}$. It is expected to be higher for copper because, in addition to its higher thermal conductivity, its low fluence absorptivity is $5 \%$ vs. $13 \%$ for aluminum. However these factors alone can not produce such a difference. We observe the effects of the equation of state. Critical point parameters of copper are similar to those of aluminum $\mathrm{Cu}: \rho_{c} / \rho_{0} \sim 0.22\left(\rho_{\mathrm{c}}=2 \mathrm{~g} / \mathrm{cm}^{3}\right), T_{c} \sim 0.5 \mathrm{eV}$ 


$$
\mathrm{Al}: \rho_{c} / \rho_{0} \sim 0.19\left(\rho_{\mathrm{c}}=0.51 \mathrm{~g} / \mathrm{cm}^{3}\right), T_{c} \sim 0.5 \mathrm{eV}
$$

but the behavior of the EOS in the mixed phase region is quantitatively different. The region of negative pressure in copper (effectively material strength) is larger and the pressure amplitude is higher. For instance, an isotherm that reaches minimum pressure of $-10 \mathrm{kbar}$ in aluminum corresponds to temperature $0.23 \mathrm{eV}$, however for copper such pressure is already reached on the $0.4 \mathrm{eV}$ isotherm. Thus the removal of copper requires higher temperatures than in aluminum.

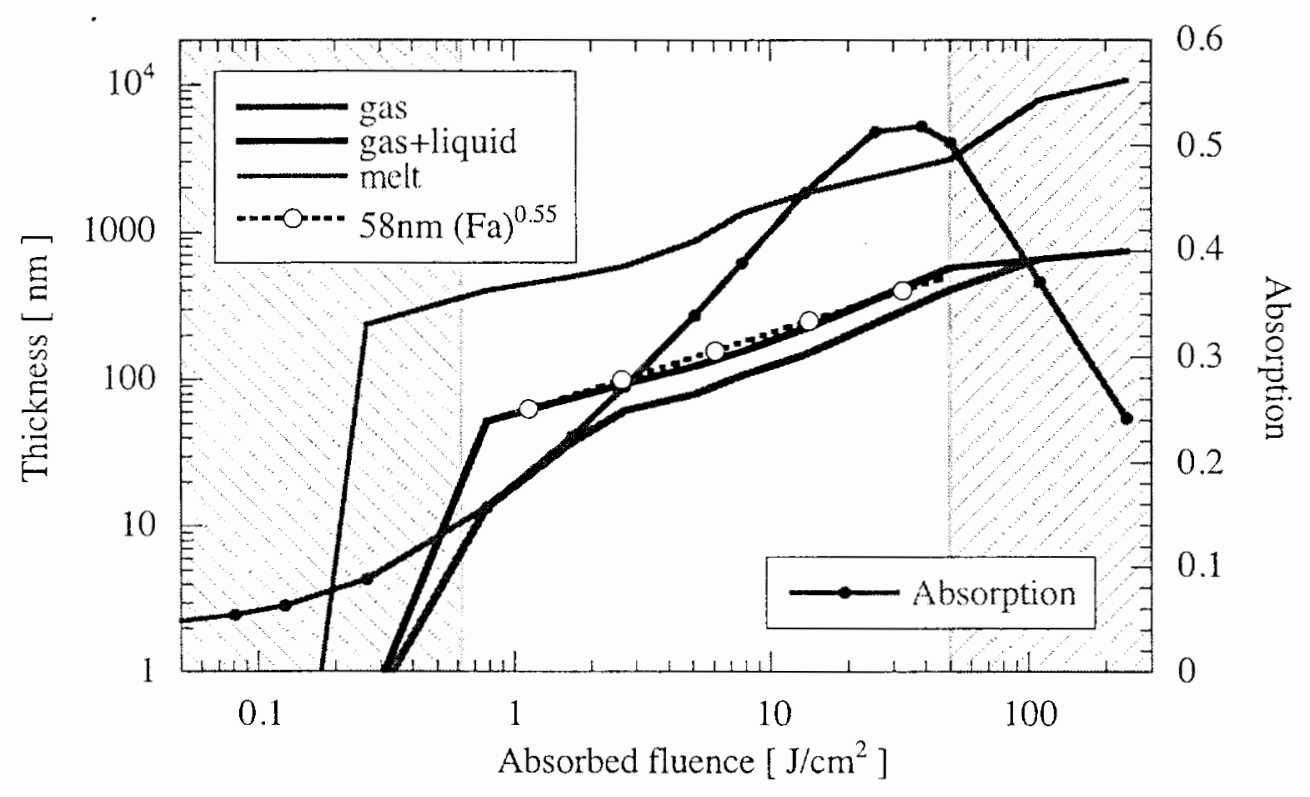

Figure 3.20: Thickness of the removed layer of copper as a function of the absorbed laser fluence. Laser parameters are wavelength: $800 \mathrm{~nm}$, pulselength: $100 \mathrm{fs}$, angle of incidence: $0^{\circ}$. The black, dashed curve with circular markers is a power fit to the removed depth (gas and liquid) in the range of absorbed fluence $0.6-40 \mathrm{~J} / \mathrm{cm}^{2}$. 
Unfortunately, the gas-liquid region in metals is not very well studied. This, in addition to the inability of the hydrocode to describe phase dynamics, makes the determination of the removal threshold not very reliable. Available experimental data for USLP ablation of copper with somewhat different laser parameters (wavelength: $780 \mathrm{~nm}$, pulselength: $150 \mathrm{fs}$ [Nolte97]) shows a two stage removal process. First, there is a slow rate removal that starts at laser fluence of approximately $0.15 \mathrm{~J} / \mathrm{cm}^{2}$ and stays within $10 \mathrm{~nm} /$ pulse until $0.5 \mathrm{~J} / \mathrm{cm}^{2}$. Then the rate increases noticeably. The authors made measurements up to $10 \mathrm{~J} / \mathrm{cm}^{2}$ fluence and fitted it to a logarithmic dependence on fluence. At $10 \mathrm{~J} / \mathrm{cm}^{2}$ they measure a removal rate of approximately $200-250 \mathrm{~nm}$. This is larger than the number we obtain using our criterion $(\sim 100 \mathrm{~nm})$, but smaller than the calculated melted depth $(\sim 550 \mathrm{~nm})$. This discrepancy only underscores the difficulty of calculation of the removal, especially in low energy regimes.

Explaining the benefits of ultrashort laser processing, we claimed that most of the deposited energy is taken away by the expanding ejecta. Using the hydrocode and our definition of the removal (removed material becomes ablation plume), this statement can be easily verified. Adding internal and kinetic energy of the ejecta and subtracting initial internal energy of the material, we obtain the amount of the energy that leaves the material with the ablation products. Figure 3.21 shows the results of such calculations carried out for the simulations presented in Figure 3.18. The plot shows that approximately $80 \%$ of the deposited energy is taken away (near the threshold it is 
approximately $50 \%$ ). Since average absorptivity is $30 \%$, only $5-10 \%$ of the laser pulse energy is left in the bulk.

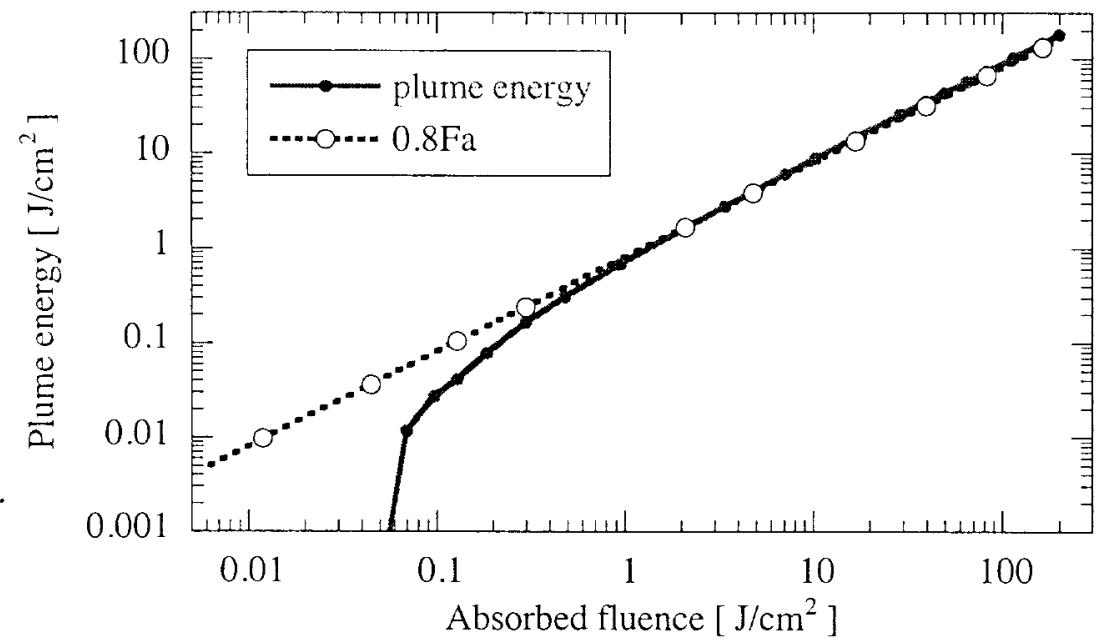

Figure 3.21: Energy of the expanding plume as a function of absorbed laser fluence. All the parameters are the same as in Figure 3.18. Black, dashed curve with circle markers shows linear dependence on absorbed fluence $0.8 \mathrm{Fa}$.

In section 3.2 ultrashort and short (nanosecond) pulse ablation were compared for the case when the removal is the same. If the same criterion is applied to these runs, we find that for USLP with fluence $5 \mathrm{~J} / \mathrm{cm}^{2}$ absorbed energy is $1.06 \mathrm{~J} / \mathrm{cm}^{2}$ and energy deposited in the bulk is $0.3 \mathrm{~J} / \mathrm{cm}^{3}$. In the long pulse case, the laser fluence was $20 \mathrm{~J} / \mathrm{cm}^{2}$, absorbed fluence was $18.65 \mathrm{~J} / \mathrm{cm}^{2}$ and deposition in the bulk was $6.8 \mathrm{~J} / \mathrm{cm}^{2}$. The energy deposited in the bulk in the long pulse ablation is much larger because a higher fluence is needed to achieve the same amount of ablation (plasma shieiding effect) and because a larger fraction of the absorbed energy diffuses in the bulk (hot plume stays near the surface for 
longer time).

\subsection{Three-dimensional evolution of the ablation plume}

The one-dimensional hydrocode gives a good description of the initial expansion stage that lasts, typically, a few nanoseconds and corresponds to a final plume thickness of a few hundred microns. However, in many applications it necessary to know the plume behavior on a much longer temporal scale. For instance, in laser film deposition the substrate for film growth is typically located centimeters away from the target. A straightforward approach to this problem would be to employ multidimensional hydrocodes such as LASNEX [Zimmerman75] and model the whole process from the beginning (USLP absorption in a thin surface layer) to the very end (long-term expansion). Unfortunately, due to the large disparity in spatial and temporal scales, this is a very challenging numerical problem (the fact that LASNEX is a classified property of the US government doesn't help either). On the other hand, at long times, the ablation plume evolution is described by ordinary hydrodynamics and should be sensitive only to integral parameters, such as laser energy deposited, amount of the material removed, etc.. It has been suggested [Singh90,Anisimov93] that a special solution of hydrodynamics [Ovsiannikov82] describes the three-dimensional stage of the expansion. Here we discuss matching of such a solution with initial conditions obtained from the HYADES calculations. 
Ultrashort laser-material interaction creates a hot, pancake-like ablation plume (Figure 3.22 ) that begins to expand after the absorption. Since it is very thin, the $z$ component of the pressure gradient is by far the largest. Material acceleration is proportional to this gradient (see equations $(2.67),(3.36)$ ). Thus the most rapid expansion occurs in the direction orthogonal to the surface. The shape of the plume changes with time and it becomes more elongated along the $z$ axis. The material evolution is adiabatic and the temperature and pressure decrease with the expansion. Once the pressure is noticeably reduced, the expansion becomes inertial (most of the internal energy is converted into kinetic) and the plume shape is preserved.

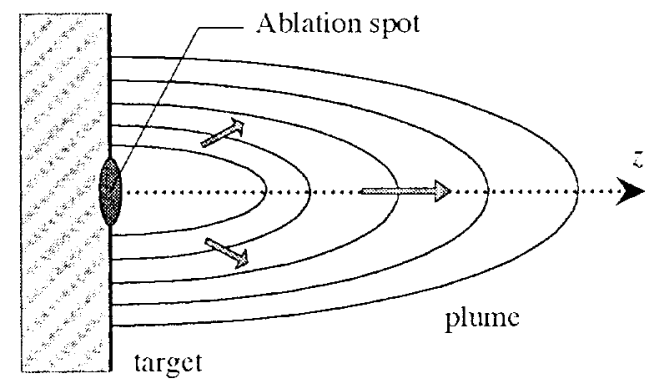

Figure 3.22: Schematic diagram of laser ablation plume expansion.

In section 3.4 we discussed a self-similar motion of an ideal gas described by the planar equations of hydrodynamics. The same type of shape preserving evolution of material parameters also exists in three dimensions [Ovsiannikov82]. The hydrodynamic solution relevant to our problem describes the expansion of an ellipsoidal gas cloud into vacuum. In this case the particle motion is given by 


$$
\begin{aligned}
& x=x_{0} \frac{X(t)}{X(0)}, \quad u_{x}=\frac{x}{X(t)} \frac{d X}{d t}, \\
& y=y_{0} \frac{Y(t)}{Y(0)}, \quad u_{y}=\frac{y}{Y(t)} \frac{d Y}{d t}, \\
& z=z_{0} \frac{Z(t)}{Z(0)}, \quad u_{z}=\frac{z}{Z(t)} \frac{d Z}{d t},
\end{aligned}
$$

where $(x, y, z)$ is position and $\left(u_{x}, u_{y}, u_{z}\right)$ is velocity. Parameters with subscript zero are initial coordinates. Self-similar evolution of the plume parameters is given by

$$
\begin{aligned}
& \rho(x, y, z, t)=M \frac{g_{\rho}\left(X_{0}, Y_{0}, Z_{0}, \gamma\right)}{X Y Z} f_{\rho}\left(R^{2}, \gamma\right) \\
& P(x, y, z, t)=E \frac{g_{P}\left(X_{0}, Y_{0}, Z_{0}, \gamma\right)}{(X Y Z)^{\gamma}} f_{P}\left(R^{2}, \gamma\right) \\
& T(x, y, z, t)=\frac{E}{M} \frac{g_{T}\left(X_{0}, Y_{0}, Z_{0}, \gamma, \mu\right)}{(X Y Z)^{\gamma-1}} f_{T}\left(R^{2}, \gamma\right) \\
& R^{2}=\left(\frac{x}{X(t)}\right)^{2}+\left(\frac{y}{Y(t)}\right)^{2}+\left(\frac{z}{Z(t)}\right)^{2}
\end{aligned}
$$

where $E$ and $M$ are the plume's energy and mass, $\gamma$ is the ideal gas exponent (ratio of specific heats) and $\mu$ is the atomic weight. The specific expressions for functions $g$ and $f$ depend on the type of self-similar solution. This can be isentropic (functions $f$ have parabolic profile) or isothermal ( $f$ is a constant for temperature, other parameters have Gaussian profile) expansion.

The solution (3.52) depicts an expansion with plume parameters constant on the ellipsoid surfaces $R(x, y, z)=$ const. Transformation of the ellipsoid with time is described by the ordinary differential equations for the functions $(X, Y, Z)$ [Anisimov93,Anisimov95]

$$
\frac{d^{2} X}{d t^{2}}=-\frac{d U}{d X}, \quad \frac{d^{2} Y}{d t^{2}}=-\frac{d U}{d Y}, \quad \frac{d^{2} Z}{d t^{2}}=-\frac{d U}{d Z}
$$


where

$$
U(X, Y, Z)=\beta(\gamma) \frac{E}{M}\left(\frac{X(0) Y(0) Z(0)}{X Y Z}\right)^{\gamma-1}
$$

Constant $\beta(\gamma)$ depends on the type of self-similar motion. Solution of (3.53) gives the evolution of the gas ellipsoid axes.

The equations (3.53) are equivalent to an equation of motion of a test particle in the three-dimensional repulsive potential $U(X, Y, Z)$, where $(X, Y, Z)$ plays the role of the particle coordinates. If we assume that the initial velocity is zero (kinetic energy of the plume is much smaller than the internal energy), the test particle's velocity increases the most along the axis with the smallest initial coordinate and vice versa. It is a mathematical expression of the fact that the plume expands the fastest along the direction where its initial dimension is the smallest (normal to the surface in the USLP ablation case). When the test particle moves sufficiently far from the initial coordinates $\left(X_{i}(t)>>X_{i}(0)\right)$, the potential is very small and the movement becomes inertial

$$
X(t) \approx v_{x}(\infty) t, Y(t) \approx v_{y}(\infty) t, Z(t) \approx v_{z}(\infty) t
$$

where $\left(v_{x}(\infty), v_{y}(\infty), v_{z}(\infty)\right)$ are components of the particle's velocity far from the origin. This means that in the final stage the gas ellipsoid's axes increase at a fixed rate. Thus the plume expands, but the shape is preserved and determined by the relative magnitude of the components of the final velocity $\left(v_{x}(\infty), v_{y}(\infty), v_{z}(\infty)\right)$. For the plume to be elongated in the direction orthogonal to the surface, we must have 


$$
v_{z}(\infty)>>v_{y}(\infty), v_{x}(\infty)
$$

This happens when

$$
Z(0) \ll X(0), Y(0)
$$

as is the case for USLP ablation.

There are experimental and theoretical indications of the applicability of the expanding gas ellipsoid model. According to (3.51) the velocity components of the particles must be linear functions of their respective coordinates. This is, in fact, the type of behavior we observe in HYADES calculations. Figure 3.23 shows a plot of the material velocity at several different times after the ablation of aluminum with a moderate energy USLP.

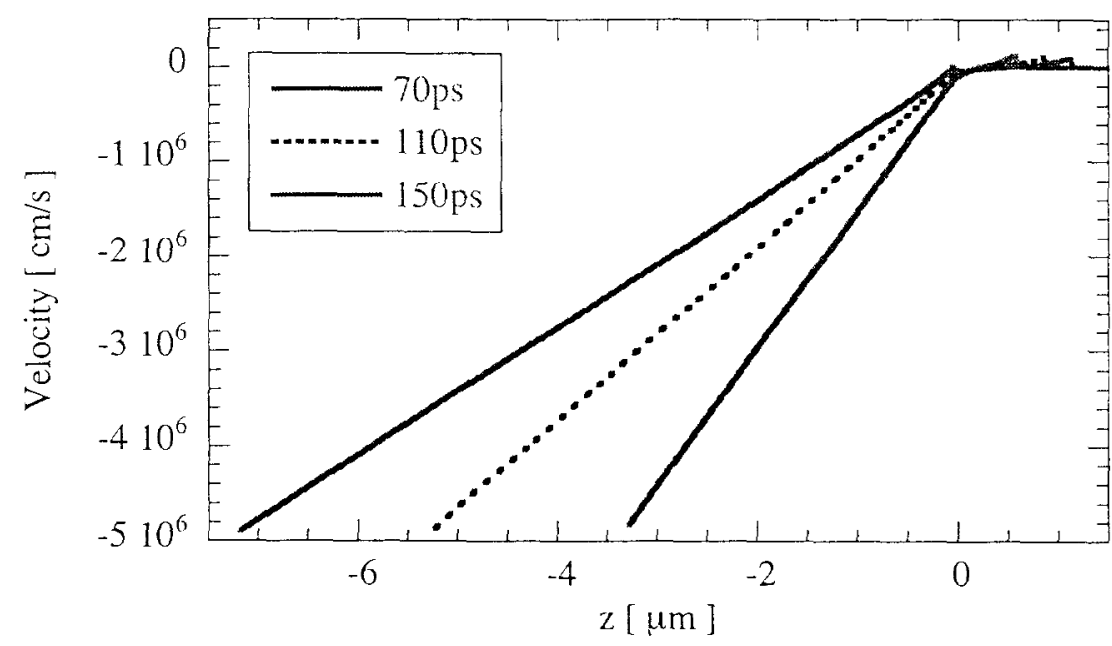

Figure 3.23: Plot of material velocity versus distance at different time. Laser parameters are the same as in Figure 3.13 (800nm wavelength, $100 \mathrm{fs}$ pulsewidth, $2.5 \mathrm{~J} / \mathrm{cm}^{2}$ fluence).

The plume's velocity does have a linear dependence on the coordinate. 
Another prediction of the theory is rotation of the plume. The smaller the initial dimension, the faster the final expansion. Therefore, if the gas ellipsoid is initially not circular in the transverse crossection (equivalent to an elliptic laser spot), in the final stage of the expansion the transverse ellipse must be rotated by 90 degrees. This effect was observed experimentally in laser film deposition [Singh88], when a spot of the deposited material was elongated in the direction orthogonal to the elongation of the laser spot.

To fully benefit from the self-similar hydrodynamic solution for the expanding ellipsoid, we would like to match the solution with the output from HYADES (representing the laser created plume as a symmetric half of the ellipsoid). This is not a straightforward task. However, for one particular problem this procedure is relatively simple.

In laser film deposition it is desired to know the profile of the deposited film thickness. If we assume that once the particle reaches the deposition substrate it either sticks or bounces and never returns, the problem is equivalent to calculating an integral

$$
h\left(x, y, Z_{s u t}\right)=\frac{1}{\rho_{f}} \int_{t_{s}}^{\infty} \rho\left(x, y, Z_{s u b}\right) u_{z}\left(x, y, Z_{s u b}\right) d t
$$

where $\rho_{f}$ is the film density and $Z_{s u b}$ is the distance from the target to the substrate (in this case it is parallel to the target). Before this integral can be calculated, equations (3.53) must be solved. It is convenient to rewrite them in the dimensionless form 


$$
X^{\prime} \frac{d^{2} X^{\prime}}{d t^{\prime 2}}=Y^{\prime} \frac{d^{2} Y^{\prime}}{d t^{\prime 2}}=Z^{\prime} \frac{d^{2} Z^{\prime}}{d t^{\prime 2}}=\left[\frac{Y^{\prime}(0) Z^{\prime}(0)}{X^{\prime} Y^{\prime} Z^{\prime}}\right]^{\gamma-1}
$$

where

$$
\begin{gathered}
X^{\prime}(t)=X(t) / X(0), \quad Y^{\prime}(t)=Y(t) / Y(0), \quad Z^{\prime}(t)=Z(t) / Z(0) \\
t^{\prime}=t / t_{0}, \quad t_{0}=\frac{X(0)}{\sqrt{\beta(\gamma) E / M}} .
\end{gathered}
$$

Here $X(0)$ is the largest ellipsoid dimension at the beginning of the expansion. The ratios $k_{y}(0)=Y(0) / X(0), k_{z}(0)=Z(0) / X(0)$ determine the initial shape of the ellipsoid. Time $t_{0}$ is a characteristic time of the expansion. Using HYADES calculations we can estimate it as

$$
t_{0}=\frac{X(0)}{\sqrt{\beta(\gamma) E / M}}=\frac{l}{\sqrt{\beta(\gamma) \frac{F_{p l e m e} l^{2}}{z_{\text {rem }} \rho l^{2}}}},
$$

where $l$ is the laser spot radius, $F_{\text {plume }}$ is the plume energy, $z_{\text {rem }}$ is removal depth, $\rho$ is material density. The constant $\beta(\gamma)$ is close to one [Anisimov95,Nemchinov65]. Plume energy and removal rate can be estimated using the results of the previous section

$$
z_{\text {rem }} \sim 60 \mathrm{~nm}(F a)^{0.6}, \quad F_{\text {phtume }} \sim 0.8 F a .
$$

Therefore we get

$$
t_{0} \sim 15 \mathrm{~ns}\left(\frac{R[100 \mu \mathrm{m}]}{\left(\mathrm{Fa}\left[\mathrm{J} / \mathrm{cm}^{2}\right]\right)^{1.2}}\right)
$$

Since $100 \mathrm{um}$ is a typical spot size and the dependence on the absorbed fluence is very weak, the characteristic expansion time is tens of nanoseconds. In laser film deposition the substrate is typically several centimeters away from the ablation target [Banks99]. 
Even if the plume expands with very high velocity (for the high energy case $F=700 \mathrm{~J} / \mathrm{cm}^{2}$ presented in Figure 3.8 the plume velocity at the front is approximately $9 \cdot 10^{7} \mathrm{~cm} / \mathrm{s}$ ), it would take at least $0.1 \mu \mathrm{s}$ to reach the substrate. By this time $t>t_{0}$ and the plume expansion is inertial. At this stage the ellipsoid's axes grow linearly in time; therefore the expression for the deposited film thickness can be significantly simplified. Variable $R$ can be rewritten as

$$
\begin{gathered}
R^{2}\left(x, y, Z_{\text {sub }}\right)=\left(\frac{Z_{\text {sub }} \tan \left(\theta_{x}\right)}{X(t)}\right)^{2}+\left(\frac{Z_{\text {sub }} \tan \left(\theta_{y}\right)}{Y(t)}\right)^{2}+\left(\frac{Z_{\text {suth }}}{Z(t)}\right)^{2}= \\
=\frac{Z_{\text {sub }}^{2}}{Z(t)^{2}}\left(1+k_{z}(t)^{2} \tan \left(\theta_{x}\right)^{2}+\frac{k_{z}(t)^{2}}{k_{y}(t)^{2}} \tan \left(\theta_{y}\right)^{2}\right)
\end{gathered},
$$

where

$$
k_{z}(t)=\frac{Z(t)}{X(t)}, \quad k_{y}(t)=\frac{Y(t)}{X(t)}
$$

and $\theta_{x}$ and $\theta_{y}$ are the angles between the axis $z$ and the lines crossing the center of the

\begin{tabular}{|c|c|c|c|c|}
\hline$k_{z}(0)$ & $5 \cdot 10^{-5}$ & $5 \cdot 10^{-4}$ & $5 \cdot 10^{-3}$ & $5 \cdot 10^{-2}$ \\
\hline$\gamma=5 / 3, k_{z}(\infty)$ & 160.22 & 50.683 & 16.018 & 5.0316 \\
\hline$\gamma=6 / 4, k_{z}(\infty)$ & 42.993 & 19.831 & 8.9954 & 3.861 \\
\hline$\gamma=7 / 5, k_{z}(\infty)$ & 20.756 & 11.563 & 6.3025 & 3.213 \\
\hline
\end{tabular}

Table 3.2: Ellipticity parameters $k_{i}(\infty)$ as a function of $k_{i}(0)$ and $\gamma$ calculated using equations (3.57) for a circular plume $\left(Y(t)=X(t), k_{y}(t)=1\right)$.

laser spot and lying in planes $(x, z)$ and $(y, z)$ respectively. Functions $k_{z}$ and $k_{v}$ determine the shape of the ellipsoid as it expands. At the final inertial stage they are simply 
constants. Therefore the integral (3.56) can be simplified to

$$
\begin{gathered}
h \sim \int_{t_{s}}^{\infty} \rho\left(x, y, Z_{\text {sutb }}\right) u_{z}\left(x, y, Z_{\text {sub }}\right) d t \sim \int_{t_{s}}^{\infty} \frac{f_{\rho}\left(R^{2}\right)}{X Y Z} \frac{Z_{\text {sutb }}}{Z} \frac{d Z}{d t} d t \sim \\
\sim\left(1+k_{z}(\infty)^{2} \tan \left(\theta_{x}\right)^{2}+\frac{k_{z}(\infty)^{2}}{k_{y}(\infty)^{2}} \tan \left(\theta_{y}\right)^{2}\right)^{-3 / 2} \int \frac{f_{\rho}\left(Z^{\prime-2}\right)}{Z^{\prime 4}} d Z^{\prime}
\end{gathered}
$$

Thus the film thickness is described by a simple formula independent of the details of $f_{\rho}$

$$
h\left(\theta_{x}, \theta_{y}\right)=h_{0}(0,0)\left(1+k_{z}(\infty)^{2} \tan \left(\theta_{x}\right)^{2}+\frac{k_{z}(\infty)^{2}}{k_{y}(\infty)^{2}} \tan \left(\theta_{y}\right)^{2}\right)^{-3 / 2} .
$$

Solution of the equations (3.57) connects the initial ellipticity parameters $k_{z}(0), k_{y}(0)$ with the final plume parameters [Anisimov93, Anisimov95, Komashko00]. Table 3.2 shows the relationship between initial and final plume ellipticity calculated for a gas ellipsoid that has a circular $X Y$ crossection. The thinner the initial ellipsoid, the more it is elongated in the final stage of the expansion. Deposited film thickness in this case is

$$
h(\theta)=h_{0}(0)\left(1+k_{z}(\infty)^{2} \tan (\theta)^{2}\right)^{-3 / 2} .
$$

The full width half maximum angular width of the plume is

$$
\theta_{1 / 2} \approx 2 \tan ^{-1}\left(\frac{0.766}{k_{z}(\infty)}\right)
$$

The smaller the final $k_{z}$, the higher plume directionality and the smaller the angular width.

If the initial dimensions of the USLP ablation plume were known, we could calculate the width using this model. The simplest estimate is to take the transverse size 


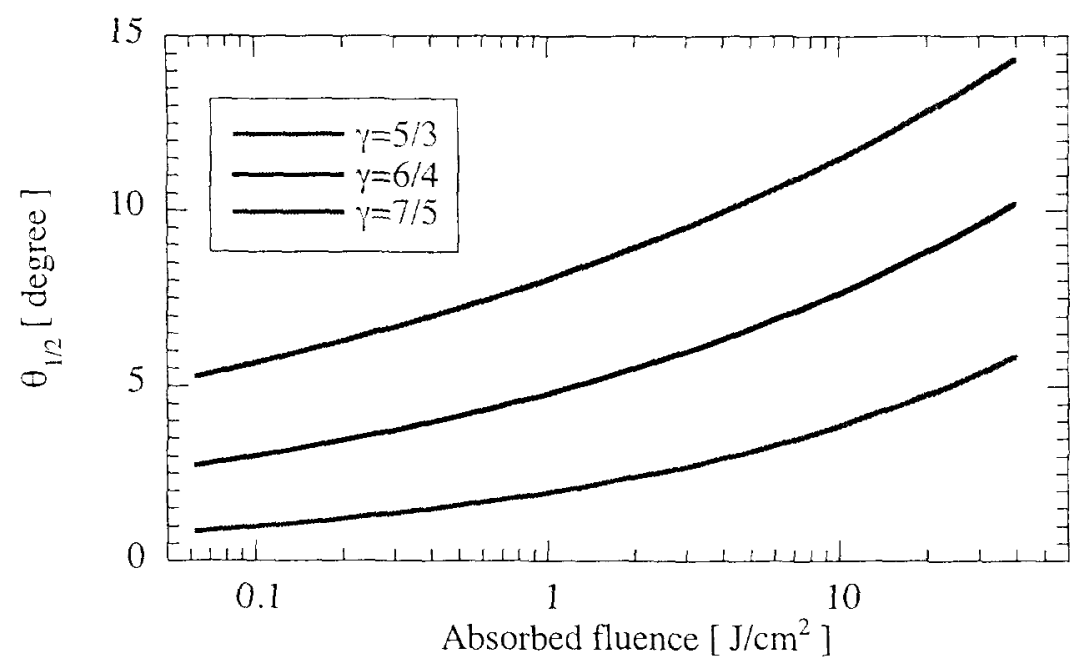

Figure 3.24: Plot of half maximum full angular width of the aluminum deposited film versus absorbed laser fluence for different gas adiabatic exponent $\gamma$. Laser parameters are the same as in Figure 3.18. Ablation spot is circular and the radius is set to $200 \mu \mathrm{m}$.

to be equal to the laser spot size and plume thickness along the $z$ axis to set equal to the removal depth. Figure 3.24 shows such a calculation for aluminum for a range of laser fluences. The ablation spot is circular with radius $100 \mu \mathrm{m}$ and the initial plume thickness is calculated with formula (3.49). Since the gas exponent is not known, calculations were done for several values of $\gamma$. The USLP plume is very directional, maximum width is $10^{\circ}$ $15^{\circ}$. More than two orders of magnitude of change in the laser fluence increase the plume width only a few times. Such a high directionality means that the actual distribution of the deposited film might be also affected by the surface quality of the target. Surface modulations change the direction of the expansion and make the distribution wider. 


\subsection{Conclusion}

Ultrashort laser-material interaction time affects not only the magnitude and the distribution of the light energy deposition, but also the material evolution after the interaction. Since the light is typically absorbed in a thin, surface layer, even moderate energy pulses have enough energy to turn this material into hot plasma. The expansion of the ablation products is fast and the plume carries away most of the absorbed energy, thus limiting energy transport into the bulk and minimizing the collateral damage. The absorption is practically instantaneous. Therefore, the width of the laser-induced pressure pulse is determined by the width of the deposition zone (instead of the laser pulsewidth). Initial pressure can be quite large; however effects like the rarefaction wave make the pressure decay quickly and become comparable to the long pulse ablation case.

The long-term effects of the USLP interaction studied in this chapter fall roughly into two categories: laser-induced pressure evolution and material ablation (removal and plume evolution). The key parameter that influences these effects is the amount of the USLP energy deposited, so we investigated material response for a variety of laser fluences.

If the laser energy is low, the material strength effects dominate the pressure pulse formation. The generated pressure pulse has a bipolar form in which a negative pressure pulse follows the leading positive peak. Since the pressure is relatively low (tens of 
kilobars), the pulse propagates with the sound speed and has a smooth form for a long time. Increase in the laser energy leads to a quick formation of a shock wave and the pulse attains a triangular form. The shocks are an important mechanism of thermal energy transport. We suggested a method for calculation of the residual heating (increase of the material temperature after the shock pulse is gone) as a function of the shock pressure and show that the residual melting begins at $660 \mathrm{kbar}$ for aluminum and $1.35 \mathrm{Mbar}$ for copper (since the method is based on widely available shock data, calculations for other materials can be easily carried out). Pressure in the Mbar range can be reached even with moderate energy USLP, therefore shocks are an energy transport mechanism competing with regular thermal diffusion. Energy loss through the heating at the shock front and action of the rarefaction wave leads to decay and spreading of the shock pressure pulses. In the weak shock regime (shock speed is approximately equal to the sound speed), the pulse width increases approximately as $\sqrt{z}$ and the pressure diminishes as $1 / \sqrt{z}$, where $z$ is the distance traveled by the pulse. Increase in the laser fluence leads to higher pressure and faster decay. Ultimately, in the multimegabar regime, the shock behavior becomes self-similar: change of the pressure profile with time is equivalent to stretching and changing the amplitude. In this case the shock is supersonic and the width and the pressure are proportional to $z$ and $1 / z$ respectively.

One of the central aspects in our study of material ablation is simulation of 
material removal. To model this very complex phenomenon, we used a removal criterion based on the use of the liquid-vapor critical point of the material. Essentially it estimates the amount of the material removed as a gas. The calculations for USPL ablation showed that, above the ablation threshold, the removal increases as a power law function of the absorbed energy. However, the most efficient processing (the largest removal per unit of laser energy) is at or near the threshold, where removal is $10-20 \mathrm{~nm}$ per pulse. Using HYADES simulations we showed that the pulselength is an important parameter. Optimal processing is with pulses shorter than one picosecond. Despite an increase in absorptivity for longer pulses, the removal actually drops.

USLP ablation creates an energetic plume that contains most of the laser deposited energy. Since plume evolution is decoupled from the laser-material interaction, the three-dimensional stage of the expansion can be described with an expanding gas ellipsoid model. Our matching of HYADES calculations with the model shows that the fastest expansion is in the direction orthogonal to the surface. Plume shape quickly becomes elongated along this direction and, as a result of this high directionality, its angular width is $10^{\circ}-15^{\circ}$ at most.

This chapter concludes our study of metals. Next, our discussion shifts to surface ablation of wide-bandgap dielectrics. Transparency of the material allows us to study three-dimensional evolution of the laser-induced pressure waves. We also investigate how the initial transparency affects laser energy absorption and transmission. 


\section{Chapter 4 Ultrashort laser pulse interaction with wide-bandgap}

\section{dielectrics}

\subsection{Introduction}

Some materials do not have freely moving electrons - their electron energy bands are either full or empty. If the gap between the lower, filled valence band and the higher, empty conduction band is large enough to inhibit thermally induced interband transitions of electrons, such materials are called dielectrics. They have low thermal and electric conductivity and typically have low absorption of light with photon energy smaller than the bandgap. Some dielectrics have a bandgap large enough to make interaction with light from most lasers very weak. Fused silica (a type of glass), for instance, is transparent to light with wavelengths down to ultra violet. It is still possible to perform processing of such material with a visible or infrared laser, because there could be other sources of absorption, for example impurities or defects, that produce initial (seed) free electrons interacting with light. These absorption centers act as energy sinks extracting energy from the laser beam and heating and ionizing surrounding material, which further amplifies absorption. At some point significant material modification like damage or ablation appears. This process usually has a threshold-like behavior: effects of the laser 
are noticeable only if the pulse energy exceeds a certain material and laser specific threshold.

For long pulses this phenomena is thermally driven and the ablation threshold fluence $F_{\text {ih }}$ varies as a power law function of pulse length $\tau^{m}$, where $m$ is close to 0.5 [Wood86,Campbel190,Feit01]. Indeed, energy deposition through some absorption point center results in a heating of volume $l^{3}$, where $l \sim \sqrt{\tau}$ is thermal diffusion length. Assuming heated material begins to absorb laser energy, the total energy deposited by the laser beam is on the order of $F l^{2}$. Thus the peak temperature is proportional to $F / l$. If ablation appears once some critical temperature is reached, formula for threshold fluence is obtained

$$
F_{t h} \sim T_{c r} l \sim l \sim \tau^{1 / 2}
$$

When a material is processed with a laser, some of the beam energy diffuses in the bulk and never participates in the ablation. Unfortunately this energy is worse than wasted - it has a negative effect on the quality of material processing. Energy deposition in the zone around the ablation spot may induce thermal and/or mechanical damage. Effects like melting, cracking, irregular ablation patterns reduce processing precision.

Processing with USLP systems can significantly alleviate this problem. The threshold fluence is smaller for shorter pulses, therefore there is less energy available for generation of deleterious effects. Also hot material is ejected before laser deposited 
energy can diffuse into the bulk, further reducing collateral damage. The high power of ultrashort pulses yields another benefit - intensity dependent nonlinear ionization mechanisms, such as multiphoton ionization, become sufficient to generate all of the needed seed electrons. The transition from thermal regime occurs at pulselengths less than a few tens of picoseconds [Du94,Stuart95], when the transfer of energy to lattice is longer than the pulsewidth and materials response is determined by the dynamics of the electrons.

Since the photoionization rate depends on the intrinsic properties of the material, random effects (defects, impurities, etc.) are not important and the ablation process becomes deterministic and attains higher processing quality. Figure 4.1 demonstrates examples of processing with conventional and USLP laser systems. For long pulses melting and irregular ablation is clearly visible, whereas ultrashort pulses create clean and smooth ablation craters. Long pulse absorption produces higher thermal stresses and, since many dielectrics are brittle, material cracking is very probable (visible on the picture).

Precision ablation and small collateral damage make USLP systems an attractive tool for a variety of applications, especially in medicine [Neev96,Loesel96, Loesel98, Oraevsky96,Voge194a-b,Hu96]. A number of studies of laser ablation were performed for aqueous dielectrics and especially water, since it is a good model for biological tissue [Juhasz96,Hammer96,Vogel96a,Glezer97]. 

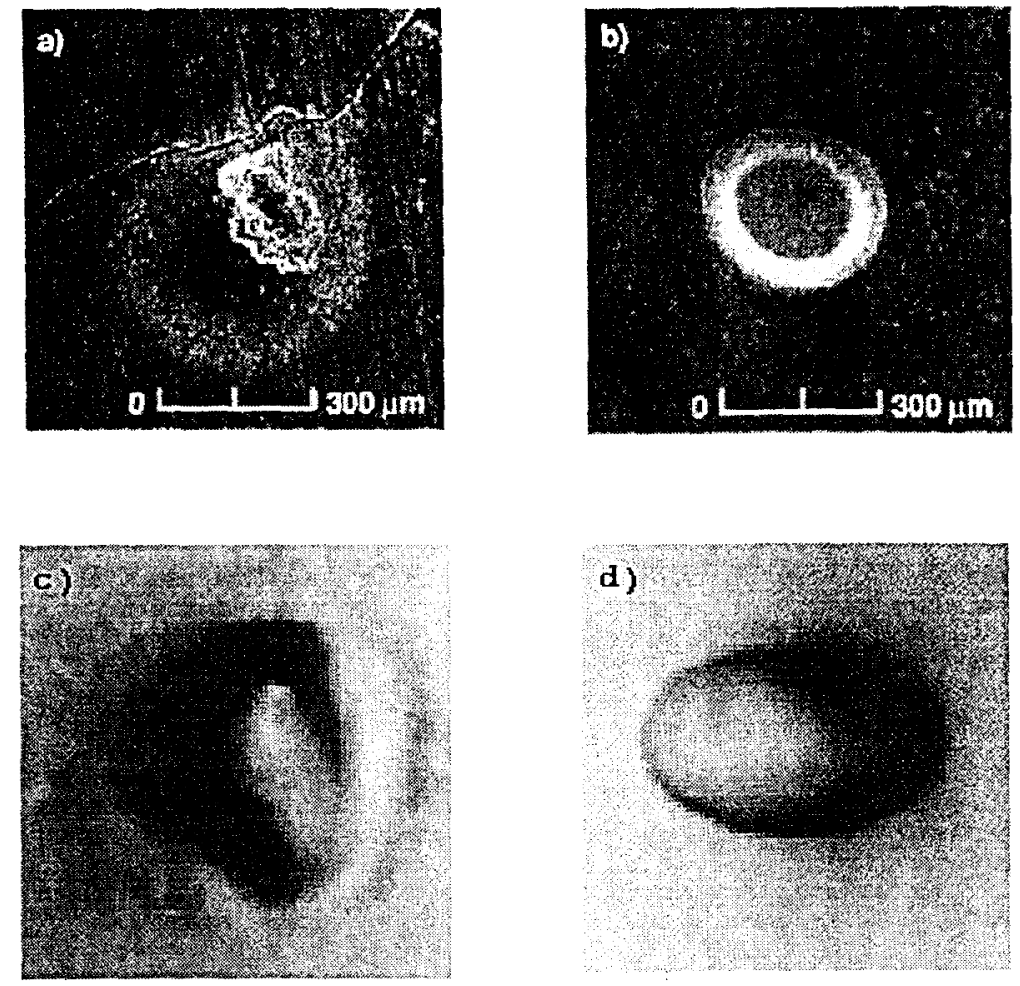

Figure 4.1: Laser ablation of dielectrics with $1053 \mathrm{~nm}$ laser. (a,b): Drilling of enamel (tooth), spot size is $300 \mu \mathrm{m}$; (a): nanosecond pulses (ablation threshold $=30 \mathrm{~J} / \mathrm{cm}^{2}$, pulse length $\tau=1.4 \mathrm{~ns}$ ), (b): ultrashort regime (ablation threshold $=3 \mathrm{~J} / \mathrm{cm}^{2}, \tau=350 \mathrm{fs}$ ) [Perry99a]. $(\mathrm{c}, \mathrm{d})$ : Craters produced in uncolored collagen gel, irradiated area is $2 \cdot 10^{-3} \mathrm{~cm}^{2} ;$ (c): nanosecond pulses ( $\tau=\operatorname{lns}$, pulse energy $E=41.7 \mathrm{~mJ}$ ), (d): ultrashort regime ( $\tau=300 \mathrm{fs}$, $\mathrm{E}=4.4 \mathrm{~mJ})[$ Oraevsky96].

One of the major concerns in this problem is the shock wave generated by the ablation process. Effects of material heating are usually easy to identify, but the pressure can affect the tissue in a subtler, invisible way [Doukas96] even at a considerable distance from the ablation spot. There may be no obvious mechanical damage, however the shock can be strong enough to adversely modify or kill tissue cells. 
In this chapter an experimental and theoretical study of mechanical effects of USLP surface (as opposed to volumetric in other publications) ablation of water is presented. This study was a collaboration effort with Beop-Min Kim and his colleagues [Kim01,Kim02], who performed the experiment in Medical Technology Program at Lawrence Livermore National Laboratory. We begin with a description of the experimental setup and the measurement technique. The following section shows typical experimental results. Next section is devoted to the theory of the USLP interaction with dielectrics. A review of the major ionization mechanisms in the wide-bandgap dielectrics is given and the method for modeling of the interaction is described. Results of the application of this method to the simulation of the experiment are also presented. Section 4 describes measurements of the wave strength and evolution, together with theoretical modeling. Conclusion section finishes the chapter.

\subsection{Experimental setup}

Our purpose is measurement of laser ablation induced pressure evolution in water. Since pressure compresses water and changes its density and refractive index, MachZehnder [Born89] interferometer is used to observe shock wave propagation. Strength of the shock is deduced from the interferometric data. Figure 4.2 shows schematic diagram of the experimental setup. Ultrashort $800 \mathrm{~nm}$ laser pulse comes from the top and ablates water in the test cuvette. The cuvette is located in one of the arms of the interferometer. 
Interferometer light is split in test and reference beams that propagate through the corresponding cuvettes in parallel to the water surface. These recombined beams produce interferometric picture recorded by the CCD camera.

The ultrashort pulse laser system is composed of two pumping lasers, one oscillator, and a regenerative amplifier. The oscillator (Tsunami, Spectra Physics) is pumped by the frequency-doubled Nd:YAG laser (Millenia, Spectra Physics) operating in continuous mode with $5 \mathrm{~W}$ of output power. The Ti:Sapphire oscillator beam is 80 fs in pulse width, $82 \mathrm{MHz}$ in repetition rate, and $800 \mathrm{~nm}$ in wavelength.

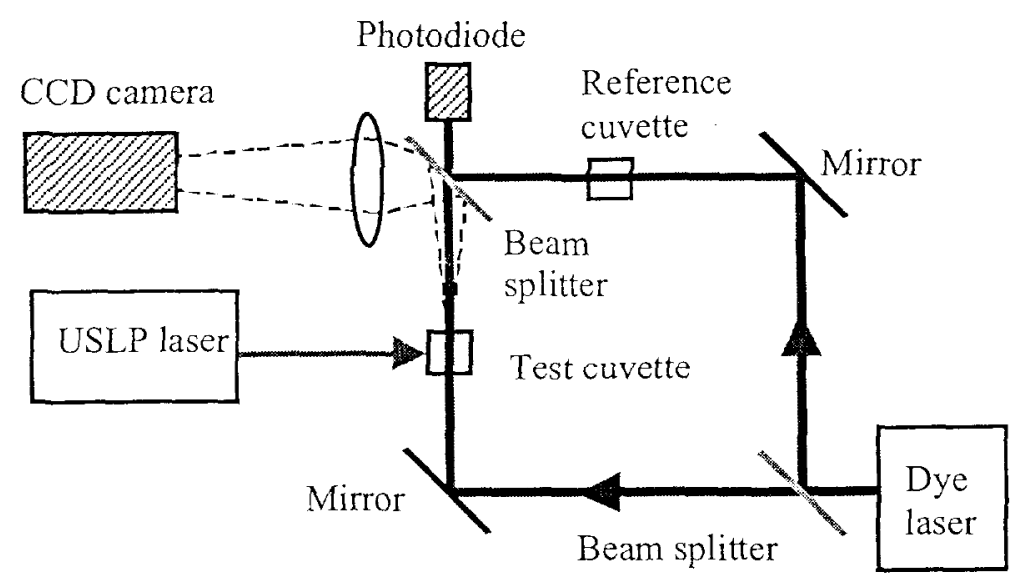

Figure 4.2: Schematic diagram of the experimental setup. Ultrashort $800 \mathrm{~nm}$ laser pulse is incident vertically at the top surface of the water contained in a cuvette. 5 ns pulse from dye laser is used to perform interferometry. CCD camera captures the picture of interference of reference and test beams.

Low energy pulse from the oscillator is amplified using a regenerative amplifier (Spitfire, Positive Light) through chirped pulse amplification process. The amplifier is pumped by 
a Q switched Nd:YLF laser (Merlin, Positive Light). Final output of the laser has maximum pulse energy of $1 \mathrm{~mJ} /$ pulse at $1 \mathrm{kHz}$ with wavelength of $800 \mathrm{~nm}$. The shortest pulse width is approximately $120 \mathrm{fs}$. Pulse energy was controlled by rotating the halfwave plate placed immediately before the polarizing beam splitter and a matching mirror. Longer pulse widths up to $10 \mathrm{ps}$ were obtained from the shortest by changing the path length of the beam compressor inside the regenerative amplifier. Pulse widths were measured using a scanning autocorrelator. The incoming laser beam was split into two beams with equal intensity and the path of one of the beams was adjustable. The beams were recombined within a KDP crystal generating the second harmonic at $400 \mathrm{~nm}$ in the direction of the intersection. The intensity $I$ of the second harmonic beam was measured as a function of the path difference $x$. From the full width half maximum (FWHM) of the function $I=f(x)$, the pulse width of the first harmonic laser pulse was calculated assuming a Gaussian beam profile.

Interferometry is performed using dye laser (LSI Inc.) operated at $480 \mathrm{~nm}$ and pumped with a Nitrogen laser. The 5 ns dye laser pulse was split into two beams (test and reference beams) using a 50:50 beam splitter. A $1 \mathrm{~cm} \times 1 \mathrm{~cm} \times 5 \mathrm{~cm}$ cuvette filled with purified water was inserted into the test beam and was illuminated on one of the four long sides. Another water-filled cuvette was placed in the reference beam of the interferometer to compensate for the path difference. The beams recombine at another beam splitter and parallel fringes are formed in the absence of USLP generated effects. Interferometer 
pulse was delayed with respect to the ablating pulse using a digital delay generator. A variable delay time from 0 to 200 ns was chosen so that the propagation of pressure waves could be observed at various times. The actual delay was measured for each ablation pulse with a separate photodiode. The diode was illuminated simultaneously with the ultrashort laser pulse scattered from the water and the transmitted dye laser pulse. The two signals were displayed and saved in a digital oscilloscope for calculation of the actual delay times.

The water surface in the test cuvette has the form of a concave meniscus. The USLP was focused on the centrum of the meniscus and beam diameter is $56 \mu \mathrm{m}$ (full width half maximum). Interferograms of the ablation induced pressure waves were imaged on a CCD camera with $512 \times 512$ pixels, each pixel measuring $48 \mu \mathrm{m}$. The shutter of the camera was synchronized with the ablation pulse. To magnify the images, a photoobjective with $\mathrm{f}=57 \mathrm{~mm}$ with high numerical aperture was used. With magnification of 45 , the resolution of the magnified images was approximately $1 \mu \mathrm{m} / \mathrm{pixel}$. The magnification was measured by imaging a micrometer-scale resolution target. The images were processed in a computer using IPLab software. By adjustment of mirrors of the interferometer, vertical or horizontal fringes were obtained for analyses of different types of pressure waves. Recording interferograms at different time delays we could observe wave dynamics. 


\subsection{Interferometry}

Once an ultrashort laser pulse incident on water reaches certain threshold intensity, it begins active deposition of its energy. Since deposition rate is very high, pressure shock waves are generated. Interferograms provide us with information about ablation and wave dynamics. Figure 4.3 demonstrates a few typical interferograms taken after $140 \mathrm{fs}, 11 \mathrm{~J} / \mathrm{cm}^{2}$ laser pulse interacted with water. The pictures show a thin, expanding, spherical shell with a center approximately at the ablation spot. The fringe shift is caused by the higher optical density of this structure, which in turn appears because of the higher physical density created by the pressure compression.
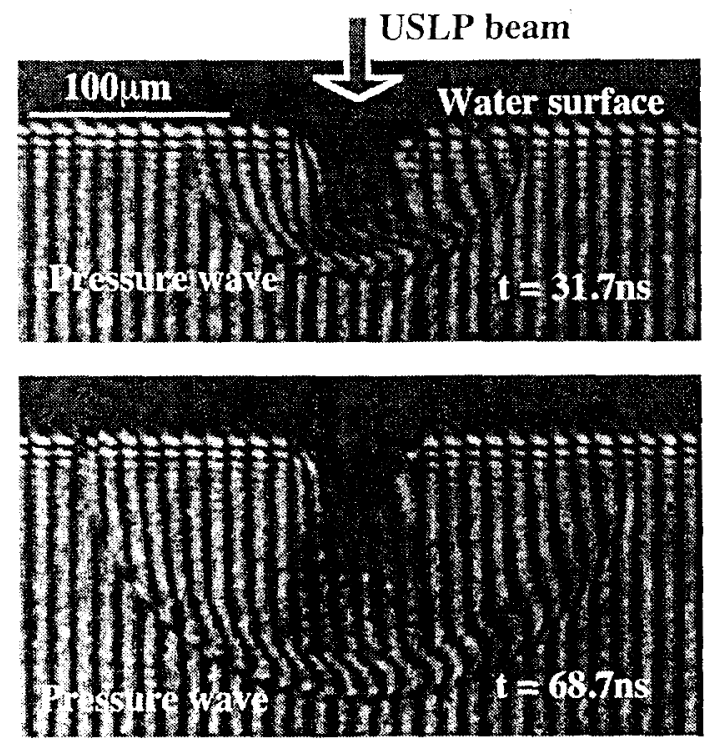

Figure 4.3: Interferograms recorded after ablation with $140 \mathrm{fs}, 11 \mathrm{~J} / \mathrm{cm}^{2}$ pulse. Thin, semicircular structure is an index change induced by shock wave propagation from the ablation spot. 
$140 \mathrm{fs}$

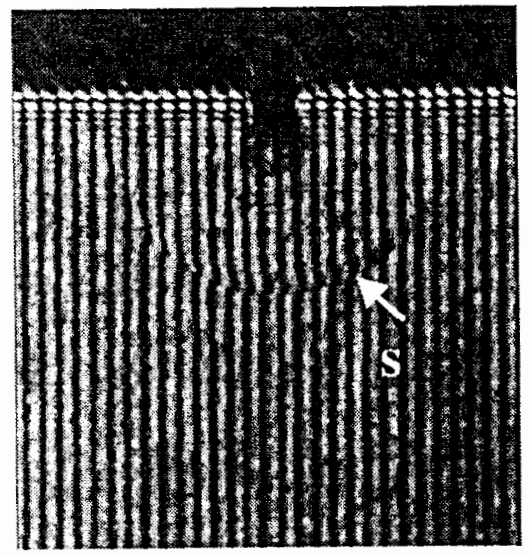

1 ps

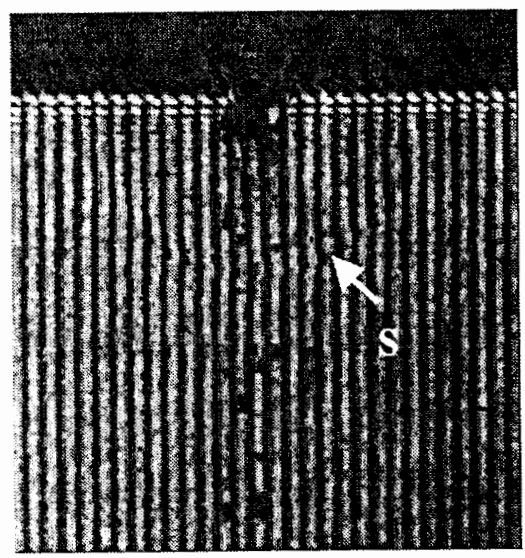

$10 \mathrm{ps}$

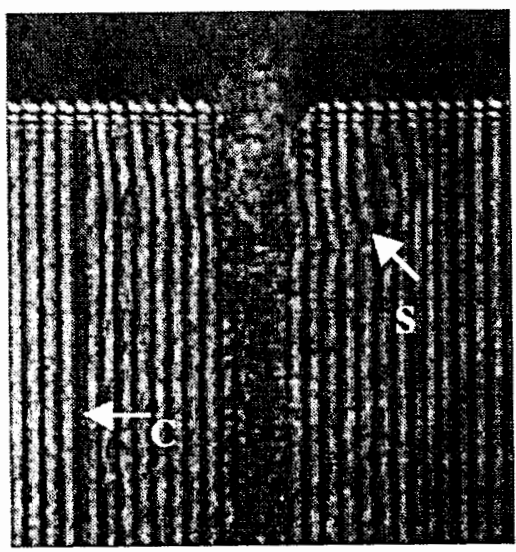

500 fs

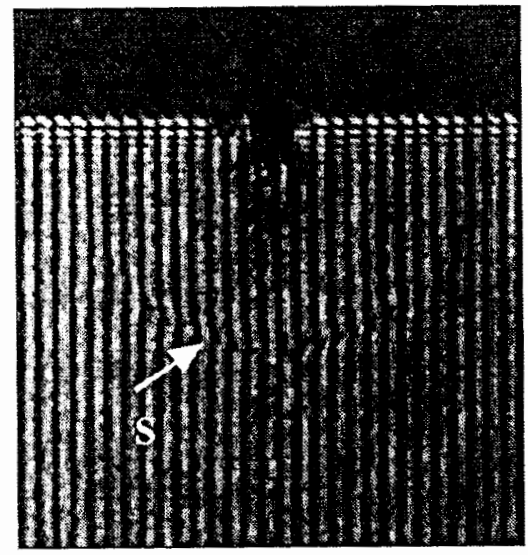

$5 \mathrm{ps}$

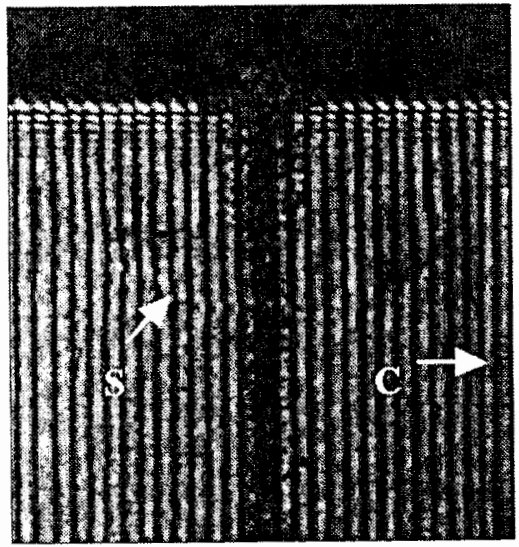

Figure 4.4: Interferometric pictures for different length of the ultrashort pulse. Laser fluence changes from several $\mathrm{J} / \mathrm{cm}^{2}$ for short pulses to tens of $\mathrm{J} / \mathrm{cm}^{2}$ for longer pulses. For pulses $1 \mathrm{ps}$ and shorter only spherical pressure wave is visible. For long pulses absorption channel along the beam path appears together with cylindrical waves (S: spherical waves, C: cylindrical waves) 
Apparently energy of $140 \mathrm{fs}$ pulse is absorbed in a thin, surface layer. The ablation spot is comparable in size to the laser spot (beam radius is $28 \mu \mathrm{m}$ ). Sound speed in water at room temperature [Gray63] is $1.48 \mathrm{~km} / \mathrm{s}$, thus a shock can propagate this distance in approximately $10-20 \mathrm{~ns}$. After that it begins to behave like a wave launched by a point source, which has a spherical form. One may notice that the wave becomes weaker close to the water surface. The pressure at the free surface must be zero, therefore the wave is weakened by this factor. Dark area in the center appears to be a region of absorption and scattering caused by heating and expansion.

Figure 4.3 describes a typical interferogram recorded after USLP ablation with pulse length below one picosecond. Further increase in pulse width produces a qualitative change in the observed interferometric pictures (see Figure 4.4). In case of short pulse we see appearance of spherical waves only, however for the longer pulses increase in the pulse energy first results in generation of cylindrical waves, apparently created by the material expansion caused by the volumetric absorption along the beam path (absorption channel is visible on the interferograms because of the light defocusing and scattering). Generation of spherical waves requires larger energy and the spherical and cylindrical wave are present simultaneously. Obviously this transformation is caused by the change in laser absorption that occurs when pulse length is increased from $1 \mathrm{ps}$ to $5 \mathrm{ps}$. 


\subsection{Theory of laser induced breakdown}

In order to explain the experimental picture, we modeled the evolution of laser absorption and electron density for a variety of USLP parameters. Since water has no free electrons, it is practically transparent initially. The response to the laser light is driven by the material ionization, which is produced mainly by two processes: nonlinear photoionization and electron avalanche ionization.

Direct photoionization of a wide-bandgap dielectric is impossible because photon energy is smaller than the bandgap, however the valence electron can transit to the conduction band after absorbing several light photons simultaneously. The number of photons $k$ needed to overcome energy barrier can be quite high. For example, an electron in fused silica needs to absorb at least six photons of $800 \mathrm{~nm}$ light. The probability of such transition is calculated with the perturbation method of quantum mechanics. The calculations show that the photoionization rate $d n_{e} / d t$ is a very nonlinear function of electric field $E$ [Keldysh65] (in this expression the field is averaged over the optical period)

$$
\frac{d n_{e}}{d t} \sim E^{2 k} \sim I^{k}
$$

The qualitative behavior of the rate is easy to demonstrate using a statistical approach [Andrews85]. A molecule of the material can be ionized if number of photons in the volume $V_{m}$ occupied by the molecule is equal or exceeds $k$ - number of photons needed 
to overcome energy bandgap. When a laser pulse with frequency $\omega$ and moderate intensity $I$ propagates through a medium with refractive index $n$, the photon density is

$$
n_{p / 2}=\frac{n I}{\operatorname{ch} \omega} .
$$

Let's do an estimate for fused silica. A molecule of fused silica $\left(\mathrm{SiO}_{2}\right)$ occupies an average volume $V_{m}=4.6 \cdot 10^{-23} \mathrm{~cm}^{3}$. Thus for $800 \mathrm{~nm}$ laser light with intensity $10^{12} \mathrm{~W} / \mathrm{cm}^{2}$ there will be $m=n_{p h h} V_{m}=10^{-2}$ photons in its volume. This is equivalent to saying that $k=6$ photons fill a volume occupied by $N=V / V_{m}=k / n_{p h} V_{m}=k / m=600$ molecules of $\mathrm{SiO}_{2}$. The statistics of coherent radiation is described by the Poisson distribution [Louisell73] and the probability to find $k$ photons simultaneously in a volume of one molecule is

$$
P(k)=\frac{m^{k}}{k !} \exp (-m)
$$

This expression is one of the principle statistical distributions [Feller62]. It describes a variety of processes, for instance radiative decay, where it is necessary to find the probability of an event occurring $k$ times within a specified opportunity frame, when it occurs $m$ times on average. In our case $m$ is very small, thus probability is proportional to a power function of intensity

$$
P(k) \sim \frac{m^{k}}{k !} \sim\left(n_{p h} V_{m}\right)^{k} \sim I^{k}
$$

The regime when the photoionization rate is proportional to $I^{k}$ is called multiphoton ionization. 
When the laser intensity increases, the electric field of the light wave begins to distort the atomic field and suppress the binding potential (see Figure 4.5). Bound electrons can become free by tunneling through the suppressed potential barrier. This is the so-called tunneling ionization. It occurs when the time $t^{\prime}$ needed by the electron to escape is much smaller than the optical cycle $1 / \omega_{0}$. In order to become free, the electron must travel a distance approximately $l=\Delta / e E$ with a typical velocity $\sqrt{\Delta / m_{e}}$, where $\Delta$ is the ionization barrier. Thus, tunneling occurs when

$$
t^{\prime} \sim \frac{\sqrt{\Delta m_{e}}}{e E}<<\frac{1}{\omega_{0}} .
$$

Introducing the parameter

$$
\gamma=\frac{\omega_{0} \sqrt{\Delta m_{e}}}{e E}
$$

we can say that tunneling ionization occurs if $\gamma<<1$ (i.e. when the field is very large). In the opposite case, $\gg>1$, the multiphoton ionization regime is valid.

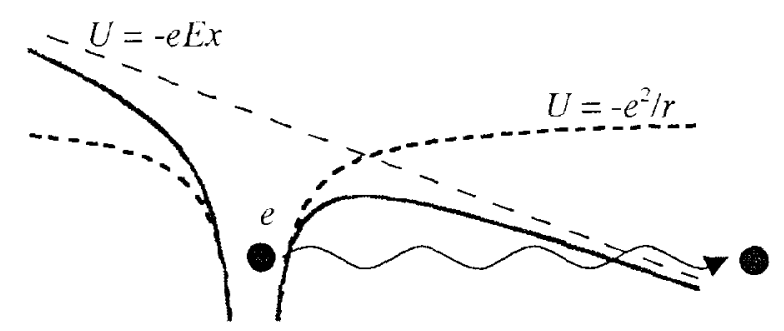

Figure 4.5: Schematic diagram of ionization through tunneling. Electric field suppresses binding potential barrier and the electron has a non-zero probability to escape.

A formula for the photoionization rate that covers both of these regimes was derived by 
Keldysh [Keldysh65]. For solid dielectrics it is given by

$$
W=\frac{2 \omega}{9 \pi}\left(\frac{m \omega}{\hbar} \frac{\sqrt{1+\gamma^{2}}}{\gamma}\right)^{3 / 2} Q\left(\gamma, \frac{\Delta^{\prime}}{\hbar \omega}\right) \exp \left\{-\pi\left(\frac{\Delta^{\prime}}{\hbar \omega}+1\right) f(\gamma)\right\}
$$

where $\Delta^{\prime}$ is effective ionization potential expressed in terms of bandgap $\Delta$

$$
\Delta^{\prime}=\frac{2}{\pi} \Delta \frac{\sqrt{1+\gamma^{2}}}{\gamma} E\left(\frac{\gamma}{\sqrt{1+\gamma^{2}}}\right)
$$

and the function $f(\gamma)$ is

$$
f(\gamma)=\left[K\left(\frac{\gamma}{\sqrt{1+\gamma^{2}}}\right)-E\left(\frac{\gamma}{\sqrt{1+\gamma^{2}}}\right)\right] / E\left(\frac{1}{\sqrt{1+\gamma^{2}}}\right),
$$

where $K$ and $E$ are complete elliptic integrals of the first kind. In (4.6) the parameter $m$ is the reduced effective mass. It is set to $m_{e} / 2$. The parameter $\gamma$ is $\omega_{0} \sqrt{m \Delta} / e E$ as above.

The symbol $\langle x\rangle$ denotes the integer part of the number $x$. The function $Q$ is given by

$$
\begin{aligned}
& Q\left(\gamma, \frac{\Delta^{\prime}}{\hbar \omega}\right)=\frac{\pi}{2 K\left(\left(1+\gamma^{2}\right)^{-1 / 2}\right)} \times \\
& \times \sum_{n=0}^{\infty}\left\{\exp \{-n \pi f(\gamma)\} \Phi\left\{\sqrt{\frac{\pi^{2}(2\langle\gamma+1\rangle-2 \gamma+n)}{2 K\left(\left(1+\gamma^{2}\right)^{-1 / 2}\right) E\left(\left(1+\gamma^{2}\right)^{-1 / 2}\right)}}\right\}\right)
\end{aligned}
$$

where $\Phi$ is Dawson's integral

$$
\Phi(x)=\int_{0}^{x} \exp \left(y^{2}-x^{2}\right) d y .
$$

Water can be represented as an amorphous dielectric [Williams75] with a bandgap of $6.5 \mathrm{ev}$. Thus at least five photons of $800 \mathrm{~nm}$ light is needed to produce one free electron. Expressing the ionization rate as 


$$
W=\sigma(E) E^{2 k}=\sigma(E) E^{10}
$$

and plotting the crossection $\sigma$ versus electric field (Figure 4.6), we can see transition from multiphoton to tunneling ionization. The parameter $\gamma$ is equal to one when $E=10^{8} \mathrm{~V} / \mathrm{cm}$. If the effect of dielectric permittivity is neglected, this field corresponds to a laser intensity of approximately $1.3 \cdot 10^{13} \mathrm{~W} / \mathrm{cm}^{2}$. Short pulses in our experiment had comparable or higher intensity, thus in the modeling the full formula (4.6) must be employed.

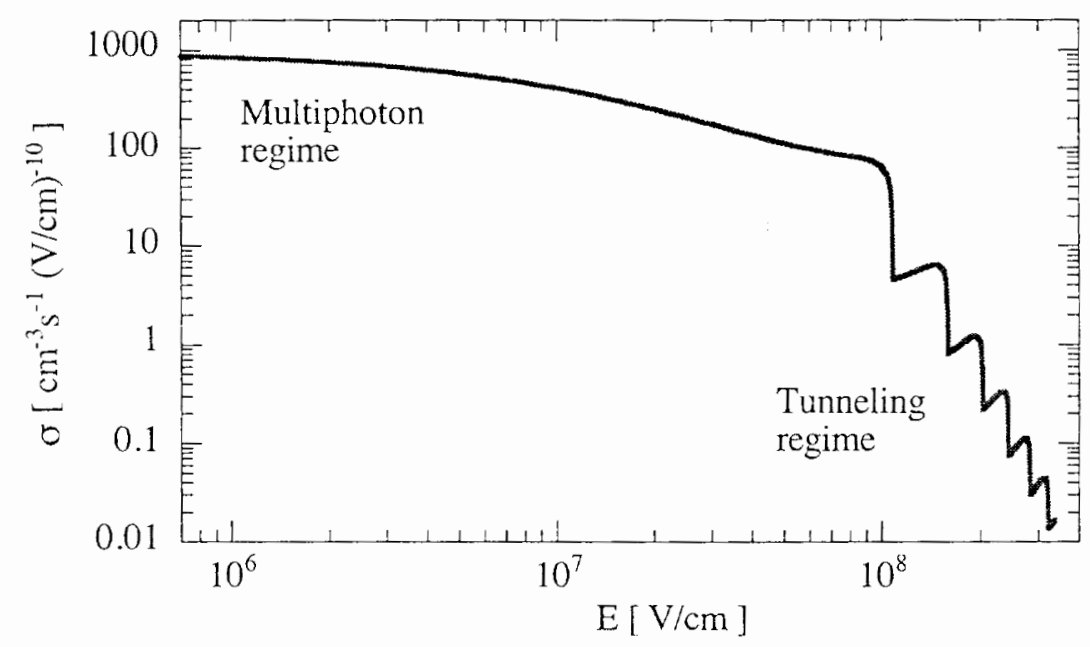

Figure 4.6: Photoionization crossection of water for $800 \mathrm{~nm}$ light as a function of electric field. In vacuum $E=10^{6} \mathrm{~V} / \mathrm{cm}$ corresponds to approximately $1.3 \cdot 10^{9} \mathrm{~W} / \mathrm{cm}^{2}$ and $E=10^{8} \mathrm{~V} / \mathrm{cm}$ corresponds to $1.3 \cdot 10^{13} \mathrm{~W} / \mathrm{cm}^{2}$.

Another important ionization mechanism is impact ionization. Once a valence electron accelerated by the laser field acquires enough energy, it can excite a valence electron across the bandgap in a collision with a molecule. The number of such events is 
proportional to the total number of free electrons

$$
W_{i m p}=\frac{d n_{e}}{d t} \sim \alpha(E) n_{e} .
$$

If $\alpha>0$, this very rapid, exponential growth of the number of free electrons is called avalanche ionization. The proportionality coefficient $\alpha$ is a function of laser field and can be calculated from laser energy absorption of electron plasma. The dielectric permittivity $\varepsilon$ is given by a Drude formula

$$
\varepsilon\left(\omega_{0}\right)=\varepsilon_{0}-\frac{n_{e}}{n_{c r}} \frac{1}{1+i v / \omega_{0}},
$$

where $\varepsilon_{0}=1.33^{2}$ is the dielectric constant of water for $\omega_{0}, v$ is the collision frequency and $n_{c r}$ is the plasma critical density for $\omega_{0}$. The laser energy deposition rate $S_{L}$ is given by the formula (2.66). Assuming no loss mechanisms and dividing it by the average electron energy $\langle\varepsilon>$ we obtain avalanche ionization rate

$$
W_{i m p}=\frac{v}{1+\left(v / \omega_{0}\right)^{2}} \frac{|E|^{2}}{8 \pi<\varepsilon>n_{c r}} n_{e} .
$$

According to modeling based on the kinetic equation [Stuart96] average electron energy can be set to one and a half of the bandgap energy. In order to compare avalanche ionization to photoionization we calculated electron density $n^{*}$ such that $W_{p h}(E)=W_{i m p}\left(n^{*}, E\right)$. If electron density is higher than $n^{*}$, avalanche ionization exceeds photoionization for that particular field amplitude. Figure 4.7 shows plot of $n^{*}$, together with photoionization rate for two collision frequencies: $3 \cdot 10^{14} \mathrm{~s}^{-1}$ and $10^{15} \mathrm{~s}^{-1}$ (typical 
collision frequency in laser induced breakdown is on the order of $10^{15} \mathrm{~s}^{-1}$ [Bloembergen74]).

Significant light absorption begins when the electron density $n_{e}$ approaches the critical density $n_{c r}$, which is on the order of $10^{21} \mathrm{~cm}^{-3}$. The graph shows that in the relevant range of intensities $n^{*}$ is noticeably smaller than the critical density, thus photoionization

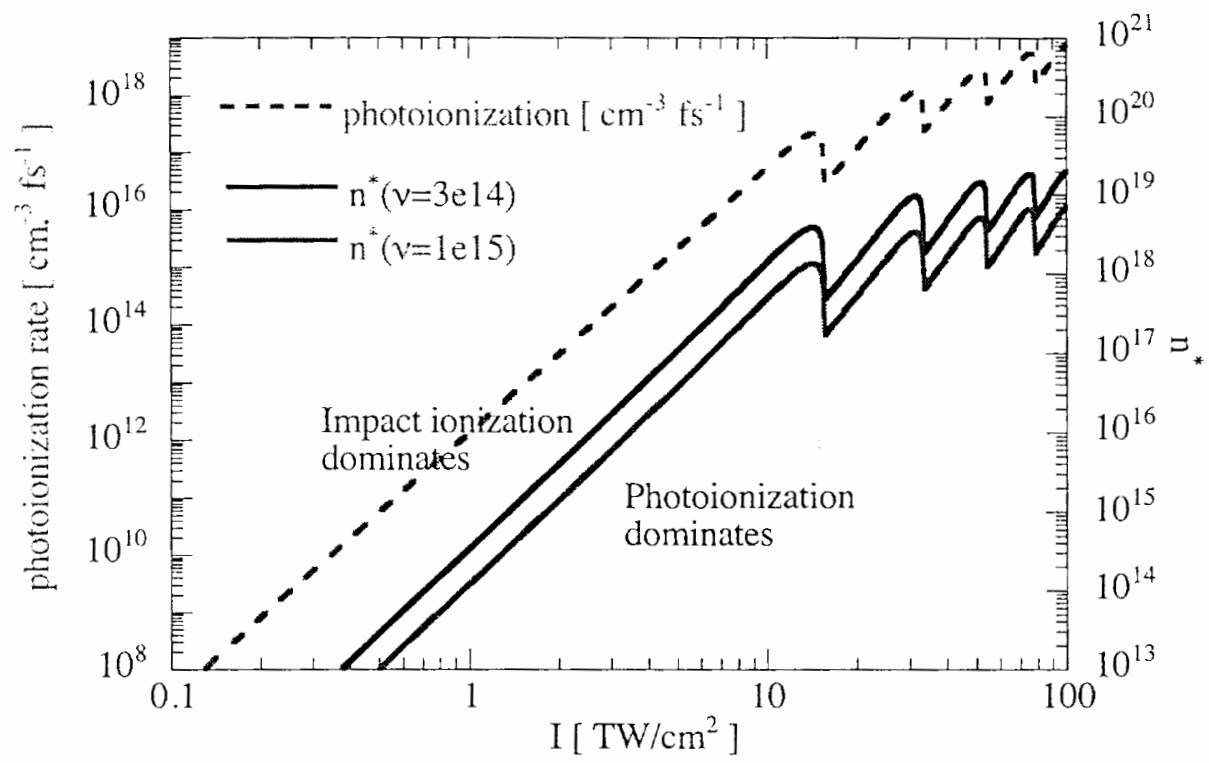

Figure 4.7: Photoionization rate and $n^{*}$ versus intensity $l=c E^{2} / 8 \pi$.

provides the initial electrons, then most of the electrons are created through impact ionization. However for high intensities photoionization itself can create significant number of electrons. According to the plot, $10^{13} \mathrm{~W} / \mathrm{cm}^{2}$ light induces ionization rate approximately $10^{17} \frac{1}{\mathrm{~cm}^{3} \mathrm{fs}}$, therefore $100 \mathrm{fs}$ pulse can create up to $10^{19} \mathrm{~cm}^{-3}$ electron density. 
Combining effects of ionization and recombination, we get an equation describing dynamics of electron density

$$
\frac{d n_{e}}{d t}=W_{p h}(E)+W_{i t r p}\left(n_{e}, E\right)-\beta n_{e}^{2}
$$

Recombination is a function of density squared because it is proportional to the number of electrons and number of ions (which is again proportional to the number of electrons). Parameter $\beta=2 \cdot 10^{-9} \mathrm{~cm}^{3} / \mathrm{s}$ was measured experimentally [Doccio88].

To implement this equation in the hydrocode would be a straightforward task. However HYADES laser propagation and absorption package can not be used in this situation. In laser induced breakdown experiments dielectrics have low initial absorption and penetration of the light wave in the material is limited only by the propagation speed. On the contrary, Helmholtz equations (2.63),(2.64), which are the basis for the HYADES light absorption modeling, neglect effects of the wave propagation, effectively making light velocity infinite. In this approximation, valid for metals, the electric field is instantaneous and any change in the input intensity is immediately felt everywhere in the simulation domain. This is not appropriate for simulation of light interaction with transparent dielectrics.

A completely different approach was adopted for simulation of laser absorption in our experiments. The problem is split in two parts. First, electron density evolution at the surface of water is calculated. The incident laser light is a Gaussian pulse 
$I(t)=I_{0} \exp \left(-4 \ln (2)(t / \tau)^{2}\right)$, where $\tau$ is full width at half maximum. The light intensity transmitted into the water is obtained from the Fresnel formulas [Jackson98]

$$
I_{s}(t)=\frac{4 \operatorname{Re} \sqrt{\varepsilon}}{|\sqrt{\varepsilon}+1|^{2}} I(t),
$$

where the dielectric permittivity as a function of time is described by the Drude formula (4.8). The electric field is calculated from the intensity as

$$
E_{s}(t)^{2}=\frac{8 \pi}{c \operatorname{Re} \sqrt{\varepsilon}} I_{s}(t)
$$

Since the dielectric permittivity is a function of density, we expressed the field as a function of time (indirectly, through $I(t))$ and electron density. Once it is substituted in the equation (4.10), we have nonlinear a differential equation describing the evolution of the electron density at the surface as a function of time. This can be solved numerically with the Runge-Kutta method [Iserlis96].

The second step consists of propagation of light intensity from position $z$ to $z+\delta z$ by subtraction of the energy expended on ionization

$$
I(z+\delta z, t)=I(z, t)-<\varepsilon>\left(W_{p h}(z, t)+W_{i m p}(z, t)\right) \delta z
$$

Step $\delta z$ is set to a small number such that the fractional change in light energy is also small. After completing this procedure we know the light intensity and field as a function of time at a position $\delta z$ deeper together with the amount of energy deposited in a layer between $z$ and $z+\delta z$. Substituting this electric field in the equation (4.10) and solving it, 
the electron density $n(z+\delta z, t)$ is obtained. Executing this algorithm of light propagation and ionization as many times as necessary, we can calculate the deposited energy density and the electron density as a function of depth, the total transmitted and reflected fluences and the temporal behavior of the laser intensity and electron density at the depth of interest.

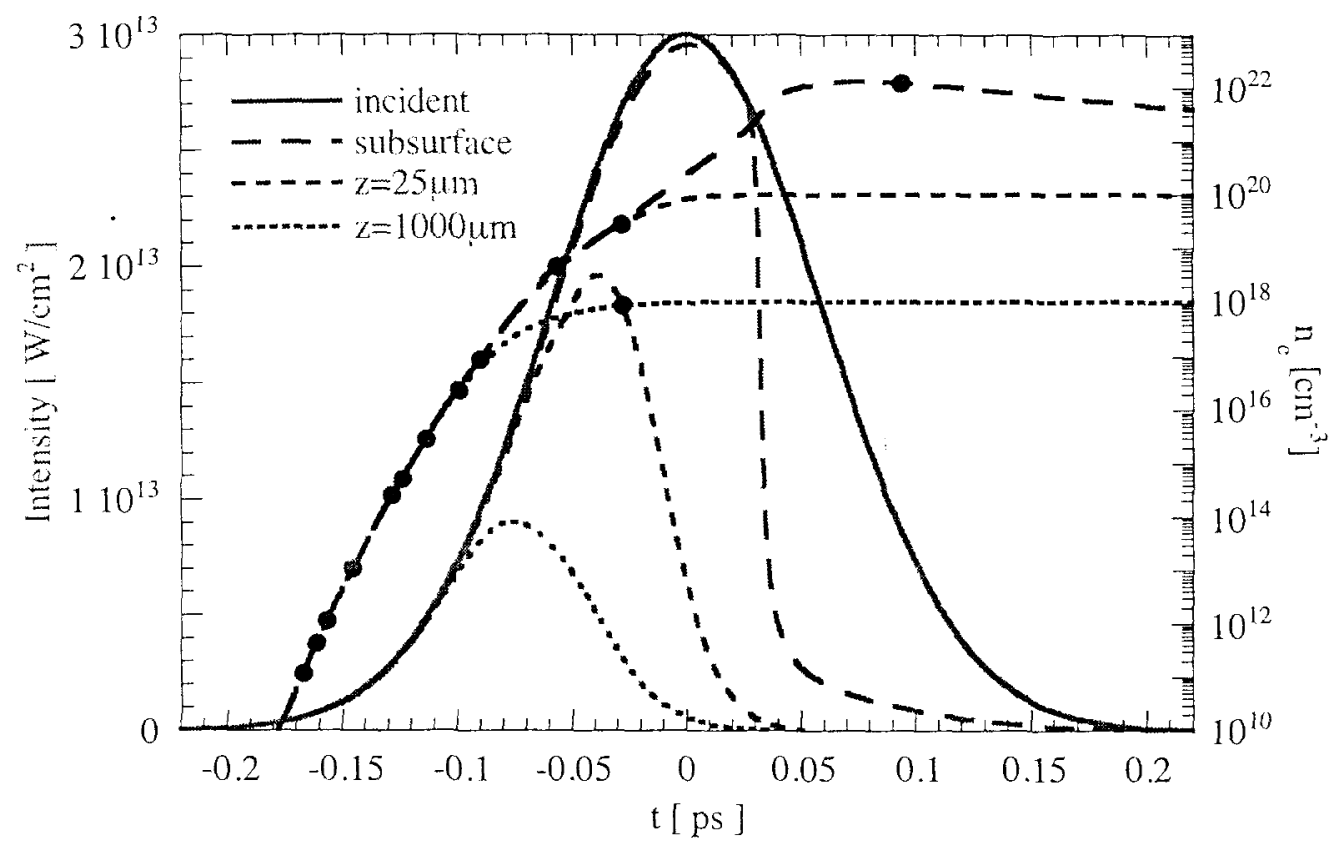

Figure 4.8: Simulation of behavior of electron density (blue lines with markers, right axis) and pulse intensity (red lines, left axis) as a function of time for three different positions: at the surface, $25 \mu \mathrm{m}$ deep and $1000 \mathrm{\mu m}$ deep for an input laser pulse with intensity $3 \cdot 10^{13} \mathrm{~W} / \mathrm{cm}^{2}$ and length $\tau=0.14 \mathrm{ps}$. Collision frequency is $v=3 \cdot 10^{14}$.

Such calculations were carried out for a number of different pulse lengths between $140 \mathrm{fs}$ and $10 \mathrm{ps}$ and for a range of intensities at each pulse width. If the fluence was low, there was practically no absorption and the laser energy propagated unchanged 
through $1000 \mu \mathrm{m}$ of water. When the intensity increased, at some point, specific for each pulse length, absorption and reflection increased sharply due to the high electron density generation at the surface and reduced energy penetration in the bulk. Figure 4.8 and Figure 4.9 show simulation results for 140 fs and 10 ps that describe this situation.

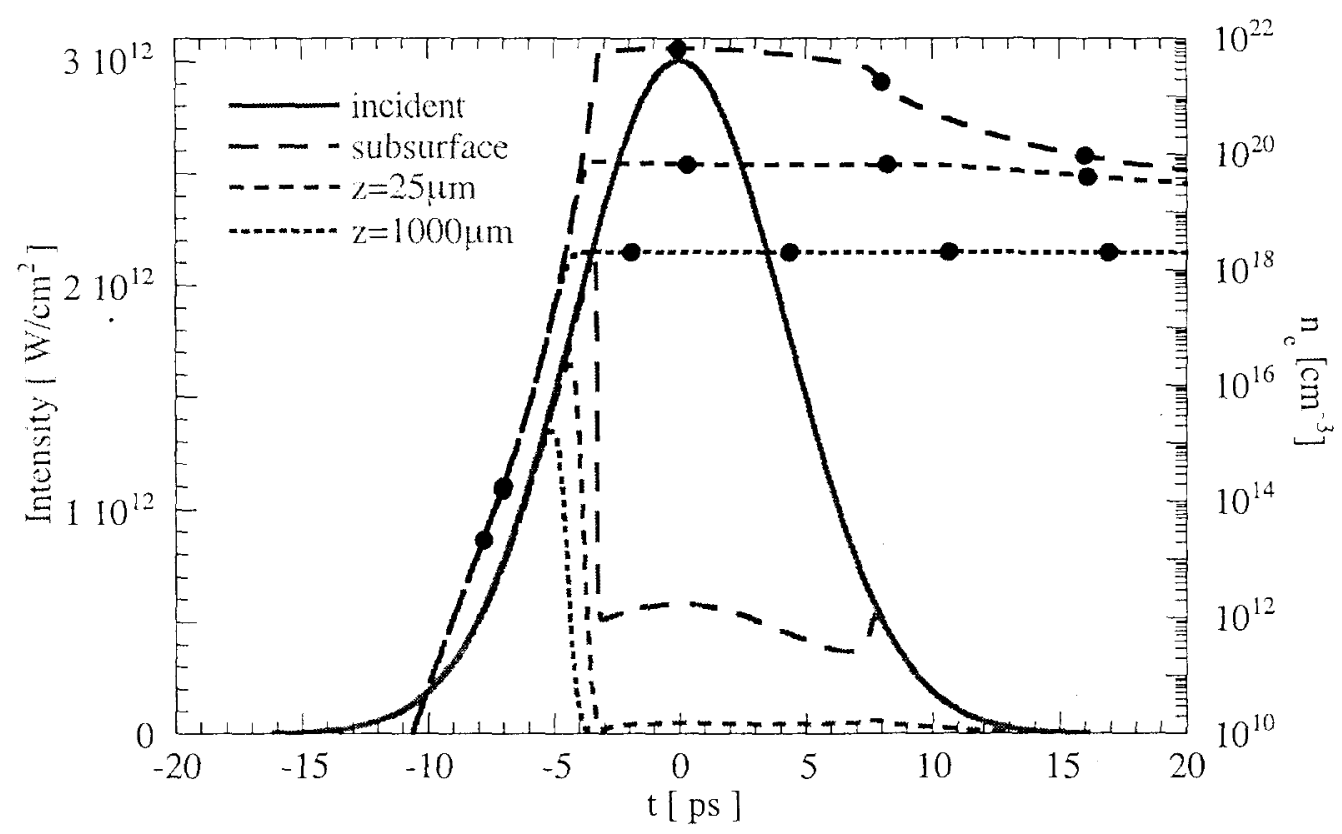

Figure 4.9: Simulation of behavior of electron density (blue lines with markers, right axis) and pulse intensity (red lines, left axis) as a function of time for three different positions: at the surface, $25 \mathrm{um}$ deep and $1000 \mathrm{um}$ deep for input laser pulse with intensity $3 \cdot 10^{12} \mathrm{~W} / \mathrm{cm}^{2}$ and length $\tau=10 \mathrm{ps}$. Collision frequency is $v=3 \cdot 10^{14}$.

The figures show the temporal evolution of pulse intensity and electron density at three different positions - immediately after surface penetration, $25 \mu \mathrm{m}$ deep and $1000 \mu \mathrm{m}$ deep. For reference, the incident intensity profile is also included. Qualitatively, the pictures are quite similar. Pulse fluences are high enough $\left(F(140 \mathrm{fs})=4.47 \mathrm{~J} / \mathrm{cm}^{2}\right.$, 
$\left.F(10 \mathrm{ps})=31.9 \mathrm{~J} / \mathrm{cm}^{2}\right)$ to generate significant electron densities. In fact, during the pulse the density exceeds $n_{c r}^{\prime}=3 \cdot 10^{21} \mathrm{~cm}^{-3}$ at the surface. If there were no collisions (in both cases $\left.v=3 \cdot 10^{1+} s^{-1}\right)$ the dielectric function $\varepsilon\left(n_{c r}^{\prime}\right)$ would vanish at this electron density and penetration of the laser radiation is arrested. The plasma effectively absorbs and reflects the radiation. For higher densities, it is also a good reflector. This is exactly what we see in the figures, once $n_{c r}^{\prime}$ is reached, i.e. transmission drops sharply. In the case of the 10ps pulse we can see this effect occur twice. The pulse is long enough for recombination to reduce the electron density below the critical density when the intensity is decreasing. As a result there is a little bump in the intensity transmission.

The modeling shows that most of the pulse energy is deposited within a surface layer $20-30 \mu \mathrm{m}$ thick with the highest absorption at the surface, where the electron density exceeds the critical $n_{c r}^{\prime}$. The region of above critical density has thickness on the order of $100 \mathrm{~nm}$ for the shortest pulses (140fs), while for longer pulses (10 ps) it decreases to 20 $30 \mathrm{~nm}$. If the laser pulse is propagated deeper into the bulk, the deposition rate decreases considerably. For the two cases presented in Figure 4.8 and Figure 4.9, the energy fraction absorbed within $25 \mu \mathrm{m}$ of the surface after propagation for $1000 \mu \mathrm{m}$ is 0.8 for $10 \mathrm{ps}$ pulse and 0.6 for $140 \mathrm{fs}$ pulse.

To understand how laser energy is divided among reflection, absorption and transmission, we performed a number of calculations for different intensities at each 
pulse length. The results indicated that the qualitative behavior is independent of the pulse width.

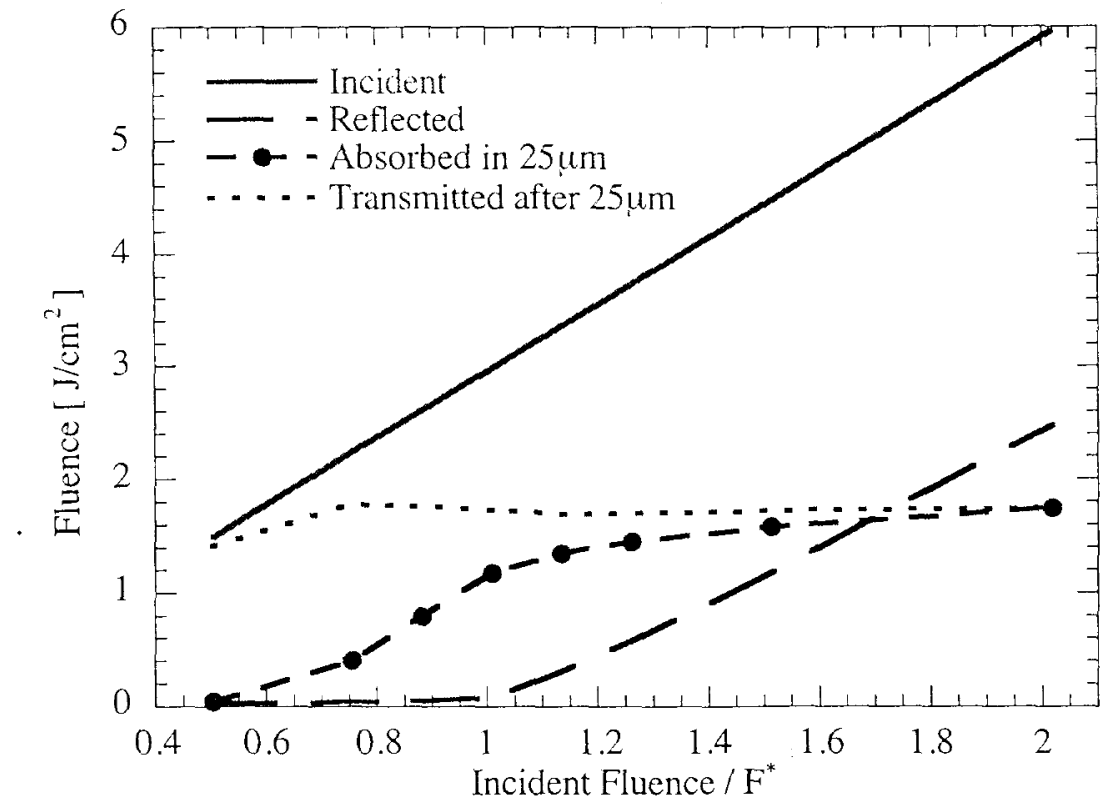

Figure 4.10: Reflected, absorbed and transmitted fluence as a function of relative pulse energy for $140 \mathrm{fs}$ and surface layer $25 \mu \mathrm{m}$ thick. The data is plotted versus normalized fluence $F / F^{*}$, where $F^{*}$ is the fluence that allows laser pulse to just produce critical electron density $n_{c r}=3 \cdot 10^{21} \mathrm{~cm}^{-3}$ at the surface. Collision frequency is $v=3 \cdot 10^{14}$.

Figure 4.10 shows results for the $140 \mathrm{fs}$ pulse length. We plot the laser fluence splitting in different energy channels as a function of the incident fluence normalized by $F^{*}=3 \mathrm{~J} / \mathrm{cm}^{2}$. We can see that absorption begins to appear for fluence $F \sim 0.5 F^{*}$. The deposited energy increases until the pulse fluence reaches approximately $F^{*}$. Afterwards it starts to saturate, whereas the energy transmitted after propagation through a $25 \mu \mathrm{m}$ surface layer stays about the same in the whole fluence range. Since energy deposition is saturated and 
transmission is not changed, all of the excess energy for incident pulse fluences above $3 \mathrm{~J} / \mathrm{cm}^{2}$ goes into reflection. Clearly $F^{*}=3 \mathrm{~J} / \mathrm{cm}^{2}$ represents a threshold value $F_{\text {th }}$; indeed it is a fluence that allows the critical electron density $n_{c r}^{\prime}$ to be reached at the surface. For longer pulses, the general picture is the same - fixed energy deposition, transmission and growing reflection for fluences above threshold. The only difference is in the value of $F_{i t}$, which increases with pulse width. For instance, the 10ps pulse has a threshold fluence $F_{t h}=10.9 \mathrm{~J} / \mathrm{cm}^{2}$. It is natural to consider the laser fluence that can generate critical electron density $n_{c r}^{\prime}$ as the ablation threshold. There is clearly a transition from no absorption and full transmission at lower fluences to a significant energy deposition at the surface for higher fluences, which should cause energetic material removal.

Experimentally, it is difficult to measure energy deposition. Typically, the ablation threshold is defined by the appearance of some observable change such as visible material damage [Stuart96]. Since post pulse examination doesn't work for water, one has to look for some transitional changes, like generation of cavitation bubbles [Noack98a] or plasma formation [Voge196b]. In our experiments, the ablation threshold was defined as the fluence at which spherical pressure waves appeared - a sign of strong laser absorption at the water surface. Using this criterion, we measured the threshold fluence for a number of pulse lengths. Figure 4.11 presents our data along with the simulation results. For short pulses $(\sim 100 \mathrm{fs})$, the ablation threshold is about $3.5 \mathrm{~J} / \mathrm{cm}^{2}$. It 
increases with pulse width to approximately $18 \mathrm{~J} / \mathrm{cm}^{2}$ at $10 \mathrm{ps}$ pulse duration. The calculated result, using the appearance of $n_{c r}^{\prime}$ as the criterion for the threshold determination, is also presented in Figure 4.11.

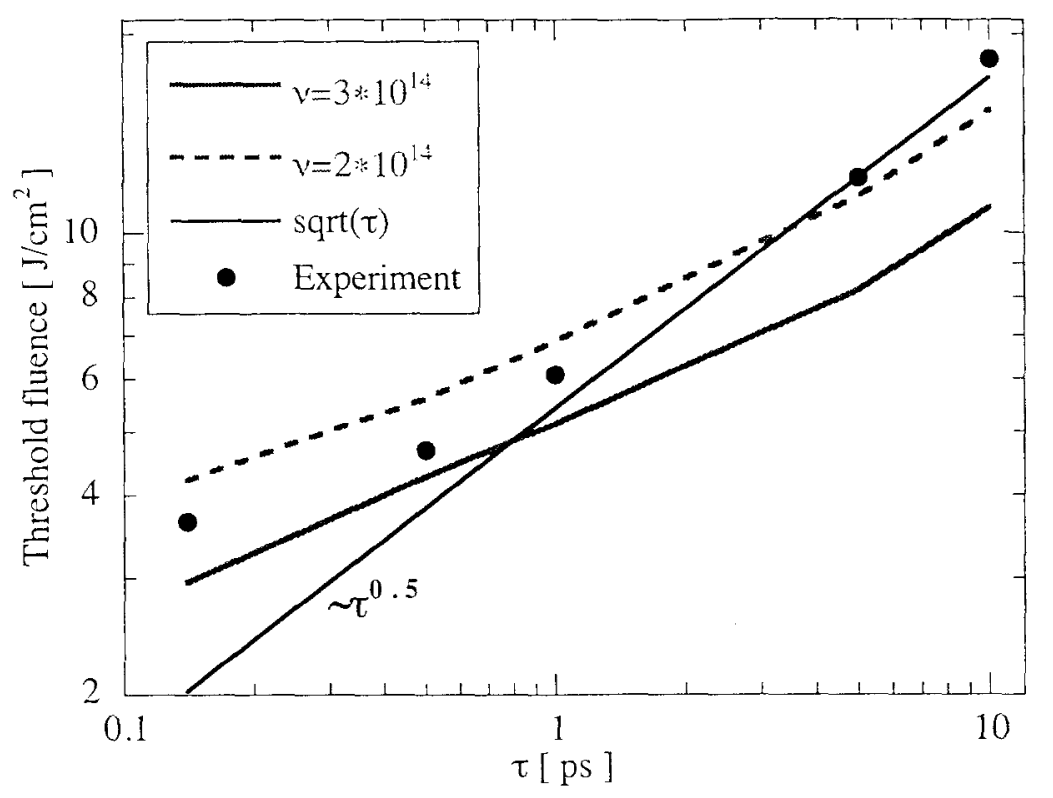

Figure 4.11: Experimental measurements and theoretical calculations of ablation threshold.

Our model has one free parameter, the collision frequency $v$. Typically, in this type of calculation [Kennedy95, Noack99] the collision frequency is set to the value proposed by Bloembergen [Bloembergen74] $v=10^{15} \mathrm{~s}^{-1}$. We adjusted this number to get the best description of the experimental result and $v=3 \cdot 10^{14} \mathrm{~s}^{-1}$ seems to be the best fit. The general behavior of the theoretical curve stays the same, when the collision frequency is adjusted; the only change is the overall magnitude.

From these curves we see that a good match can be obtained for short pulses, 
whereas the experimental curve is a steeper function of $\tau$ for longer pulses. This longer pulse disagreement can not be compensated by adjustment of the collision frequency. The discrepancy may appear because our model does not include thermal transport and electron-ion energy exchange, which lower the energy deposition rate at the surface, thus allowing higher transmission in the material and requiring a larger fluence to reach the critical density. These effects are more important for longer pulses. In fact experimental thresholds for longer pulses are well known to vary approximately in proportion to $\sqrt{\tau}$ representative of thermally controlled damage, when initial seed electrons are provided by small absorbers [Feit01]. Another reason could be the effect of hydrodynamics material expansion lowers the electron density at the surface for the longer pulses.

The following explanation, based on the simulations, emerges for the qualitative behavior of the pressure waves shown in Figure 4.4. The laser beam interacts with water generating significant electron density at the surface. This plasma acts as an energy absorber that generates spherical pressure waves, and also as a reflector that allows only the leading edge of the pulse to penetrate the surface layer. Figure 4.12 shows a calculation of energy transmission as a function of pulse length. Since the ablation threshold is an increasing function of pulse width, the transmitted energy also increases for longer pulses. For instance, in our calculations the transmitted fluence is $6.5 \mathrm{~J} / \mathrm{cm}^{2}$ for the $10 \mathrm{ps}$ pulse versus $1.7 \mathrm{~J} / \mathrm{cm}^{2}$ for the $140 \mathrm{fs}$ pulse. Therefore, the energy available for volumetric absorption along the beam path is noticeably larger for longer pulses. 


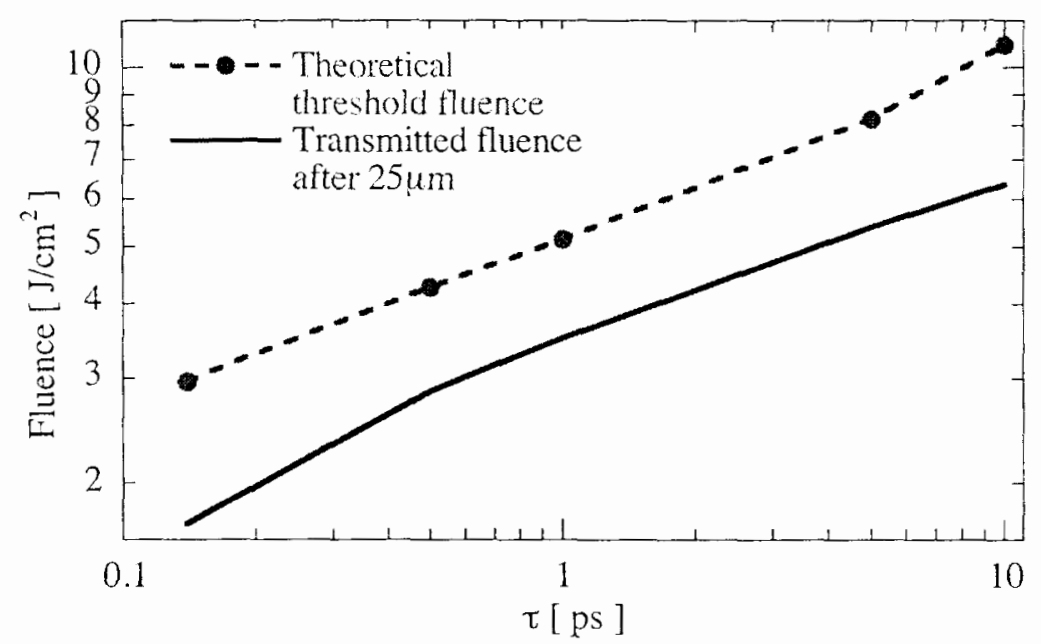

Figure 4.12: Calculated threshold fluence and transmitted fluence after $25 \mathrm{~m}$ as a function of pulse length. Collision frequency is $v=3 \cdot 10^{14}$.

As a result, we see an observable effect of this larger energy deposition, visible cylindrical pressure waves and a dark absorption channel.

\subsection{Pressure wave dynamics}

Absorption of an ultrafast laser pulse creates a localized region with high temperature and pressure. Hot material begins to expand rapidly, thus generating shock waves propagating into the bulk of the material. These waves carry part of the laser deposited energy and can affect material far away from the absorption zone. Our purpose is to characterize this shock effect by measurement of the wave strength and dynamics.

To get an idea of what we are dealing with, the initial stage of shock propagation was modeled with HYADES. From our calculation of laser absorption, we know the 
distribution of deposited energy as a function of depth. Assuming absorption occurs before any noticeable expansion, we can deduce the initial material temperature using the equation of state of water [Sesame83]. The obtained temperature profile, together with the unchanged water density of $1 \mathrm{~g} / \mathrm{cm}^{3}$ serve as initial conditions for the hydrocode. For the laser absorption case presented in Figure 4.8 , the initial surface temperature calculated this way is $1.4 \mathrm{eV} \sim 16200 \mathrm{~K}$. This corresponds to a peak pressure of approximately $330 \mathrm{kbar}$. However, the simulation shows that the high pressure does not last very long. Since this is surface ablation and hot material is free to leave the surface, a rarefaction wave immediately sets in.

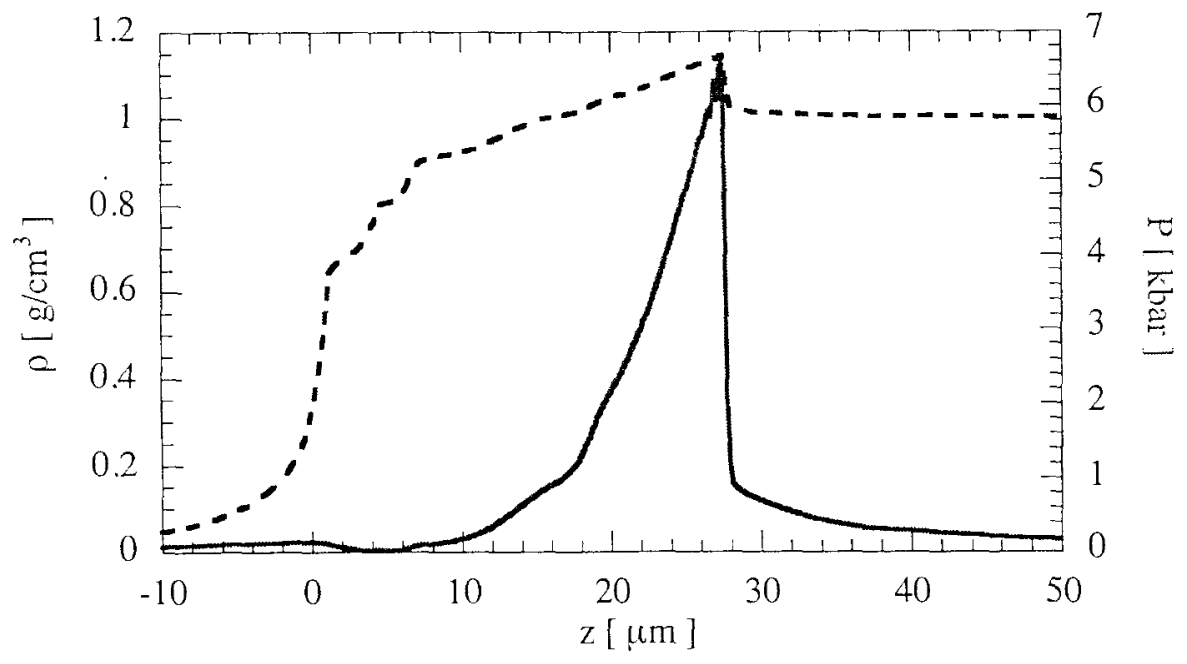

Figure 4.13: Profiles of pressure and water density calculated with HYADES. Time is lOns after light-material interaction described in Figure 4.8. Solid line is pressure, dashed line is water density. Equation of state is SESAME, table $\$ 7150$ [Sesame83]. 
After several nanoseconds, a triangular pressure pulse is formed and this pressure pulse leaves the absorption zone. Figure 4.13 shows profiles of pressure and water density $10 \mathrm{~ns}$ after the absorption. Interaction of the shock and rarefaction develops a wave with a noticeably reduced peak of $6 \mathrm{kbar}$ at this moment. Once the propagation becomes three dimensional, we can expect further lowering of pressure because of the geometrical effect. This part was done experimentally.

One of the ways to monitor pressure evolution is based on calculations of the shock speed [Noack98a]. Using interferograms taken at various delays after pulse absorption, we measured the shock position for both spherical and cylindrical waves as a function of pulse fluence and width. The time derivative of the shock radius gives its velocity. Results for the radii measurements of the spherical waves are presented in Figure 4.14. There is no significant dependence on the laser pulse width. The three curves presented are for $F=F_{t h}, 2 F_{i h}, 3 F_{t h}$. The wave propagation distance is basically a linear function of time with slightly different initial radii for the three curves. The curves all have approximately the same slope of $1.55 \mathrm{~km} / \mathrm{s}$, which is close to the sound speed in water [Gray63] of $1.48 \mathrm{~km} / \mathrm{s}$. The initial size changes from approximately $20 \mu \mathrm{m}$ for low energy $F=F_{\text {th }}$ to $40 \mu \mathrm{m}$ for the highest laser fluence. Since the laser pulse has a Gaussian spatial profile, higher beam energy makes the ablation spot larger, thus increasing the shock formation zone. Supersonic propagation during the initial stage also contributes to this change. Similar behavior was observed for the cylindrical waves - a constant 
propagation velocity close to the sound speed with an initial shock radius approximately equal to $30 \mu \mathrm{m}$ (close to the beam radius).

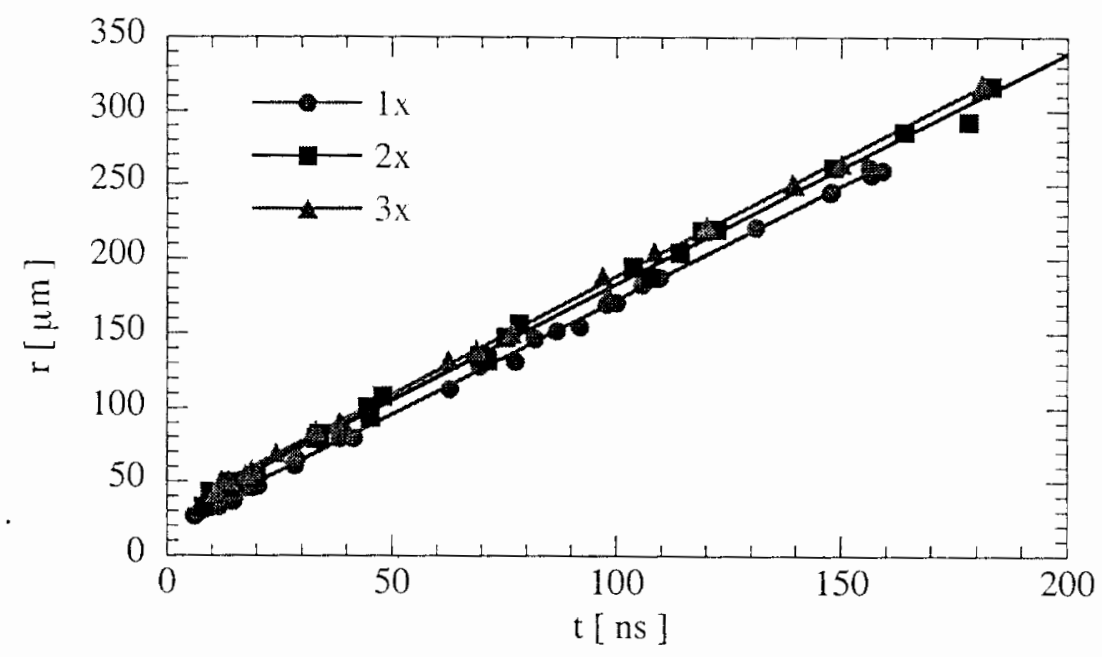

Figure 4.14: Spherical pressure wave position as a function of time. Pressure wave generated by more intense energy is initially more advanced.

This behavior is indicative of the wave strength. Using the equation of state for water [Rice57] and jump conditions at the shock wave, the pressure is related to the shock speed by [Vogel96a]

$$
P=P_{0} x\left(10^{\frac{x-1}{\beta}}-1\right)
$$

where $P_{0}$ is $76.81 \mathrm{kbar}, x=u / c, u$ is the shock speed, $c=1.483 \cdot 10^{5} \mathrm{~cm} / \mathrm{s}$ is the sound speed in water and $\beta=17.06$. When the shock speed is close to the sound speed, the formula simplifies to

$$
P \approx 10.37 \mathrm{kbar} x(x-1) \text {. }
$$


If the wave pressure is $6 \mathrm{kbars}$ or less, the shock wave speed is below $1.4 \mathrm{c}$ and decreases rapidly with velocity. At this point velocity measurement error becomes comparable to the expected pressure induced velocity variation. Therefore, methods of pressure determination based on observation of the wave speed [Noack98a] do not work in this weak shock regime. Because pressure is always a strong function of density, which also directly affects the optical properties of water, we rely on interferometry to get more sensitive measurements of wave strength. We would like to emphasize that a major factor responsible for putting pressure generation in this weak shock regime is the configuration of ablation. If laser induced breakdown occurs inside of the material, ablation products are confined, and pressure pulses are stronger and last longer. On the other hand, for surface ablation, hot material is quickly ejected, taking with it a significant portion of the laser deposited energy and thus weakening the shock wave.

The interferograms provide us with information about the refractive index change, which can then be converted to pressure data using an appropriate formula for optical properties of water as a function of its state [Thormahlen85]. For 480nm light we have

$$
\begin{aligned}
\delta n & =P\left(1.154 \cdot 10^{-5}-6.755 \cdot 10^{-8} d T\right)+1.928 \cdot 10^{-9} P^{2}- \\
& -9.083 \cdot 10^{-5} d T-1.954 \cdot 10^{-6} d T^{2}+8.916 \cdot 10^{-9} d T^{3},
\end{aligned}
$$

where $P$ is pressure in bars and $d T$ is temperature change in degrees Celsius from room temperature of $20^{\circ} \mathrm{C}$. HYADES simulations show that a shock with several kbars pressure raises temperature of water by a few degrees, therefore one can omit the temperature 
dependence. Since the quadratic pressure term is relatively small, the change of refractive index is practically a linear function of pressure

$$
\delta n=1.154 \cdot 10^{-5} P \text {. }
$$

Theoretically, in the case of radial symmetry, one can recover optical properties for the whole wave. However this requires specialized software, which we did not have. Instead we adopted a simple method that allows us to estimate peak pressure and its evolution from several easily measurable wave parameters. Figure 4.15 shows an interferogram of a spherical wave. We can see that the outer shock radius $R$, maximum phase shift $\delta \varphi_{\max }$ and position of the maximum phase shift $x_{m}$ can be easily identified.
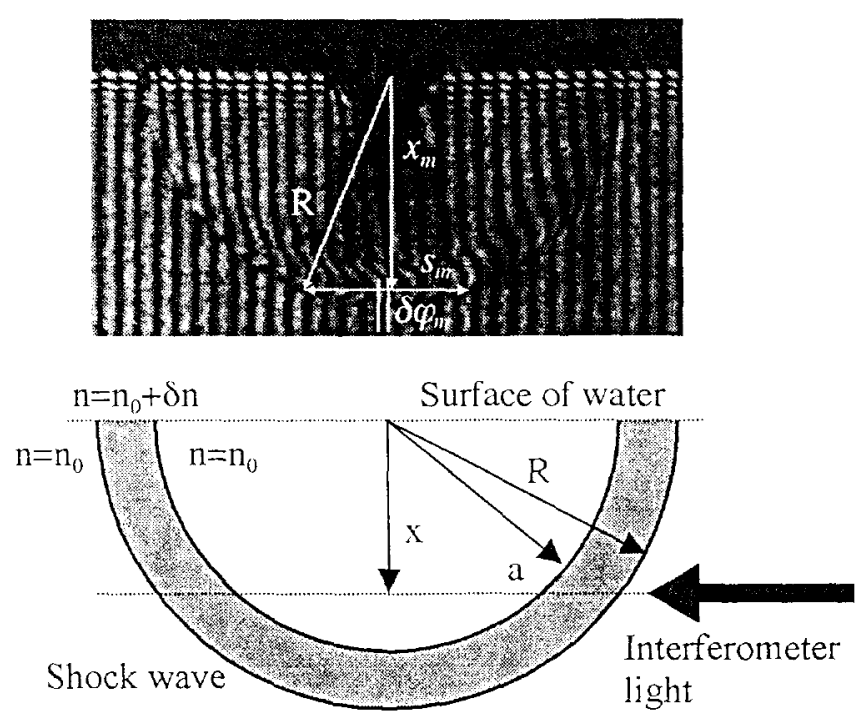

Figure 4.15: Typical interferogram and parameters used for deducing the peak pressure. Shock wave is assumed to be a thin spherical shell that induces change of refractive index $\delta n$ only inside the thin shell. $R$ is outer and $a$ is inner radii of the shell. $x_{m}$ marks distance from the water surface to the location of the point of maximum phase shift $\delta \varphi_{m}$. 
For a simple dependence of refractive index on radius these parameters can be used to calculate the maximum change in optical properties. Our modeling with the hydrocode (Figure 4.13) shows that a thin spherical shell with a triangular pulse profile would be a very reasonable assumption for the form of the refractive index. Therefore, phase shift $\delta \varphi$ is calculated as

$$
\delta \varphi(x, a, R)=\frac{4 \pi}{\lambda} \int_{\max (x, a)}^{R} \delta n(r, a, R) \frac{r d r}{\sqrt{r^{2}-x^{2}}}
$$

where $\delta n$ is the refractive index change, $a$ is an inner wave radius, $\lambda$ is interferometer wavelength and $x$ is distance from the surface to the point of phase measurement. Assuming the following variation of refractive index as a function of radius

$$
\delta n(r)=\left\{\begin{array}{cc}
\delta n_{\max } \frac{r-a}{r} \frac{R}{R-a}, & a \leq r \leq R \\
0, & r<a, r>R
\end{array},\right.
$$

we find the relationship between $\delta \varphi_{\max }$ and $\delta n_{\max }$ :

$$
\delta n_{\max }=\frac{\lambda \delta \varphi_{\operatorname{mix}}}{2 \pi s} f(s / 2 R),
$$

where $s=2 \sqrt{R^{2}-x_{m}{ }^{2}}$ is easily measured in the case of spherical geometry and $f(s / 2 R)$ is a function depending on details of the integration. This function increases monotonically from 1.0 for thick pressure shells $(a<<R, s \sim R)$ to 1.5 for thin shells $(a \sim R, s<<R)$. Since the interferograms show that the latter is practically always the case, maximum refractive index change is calculated with the value of this function set to 1.5 . It is interesting to note that if we assumed a constant rectangular profile of $\delta n(r)$, this function would have 
been equal to one identically.

Figure 4.16 shows the calculated spherical wave pressure as a function of radius for $140 \mathrm{fs}, 500 \mathrm{fs}$ and $1 \mathrm{ps}$ pulses. The pressure is strongly dependent on fluence, but only
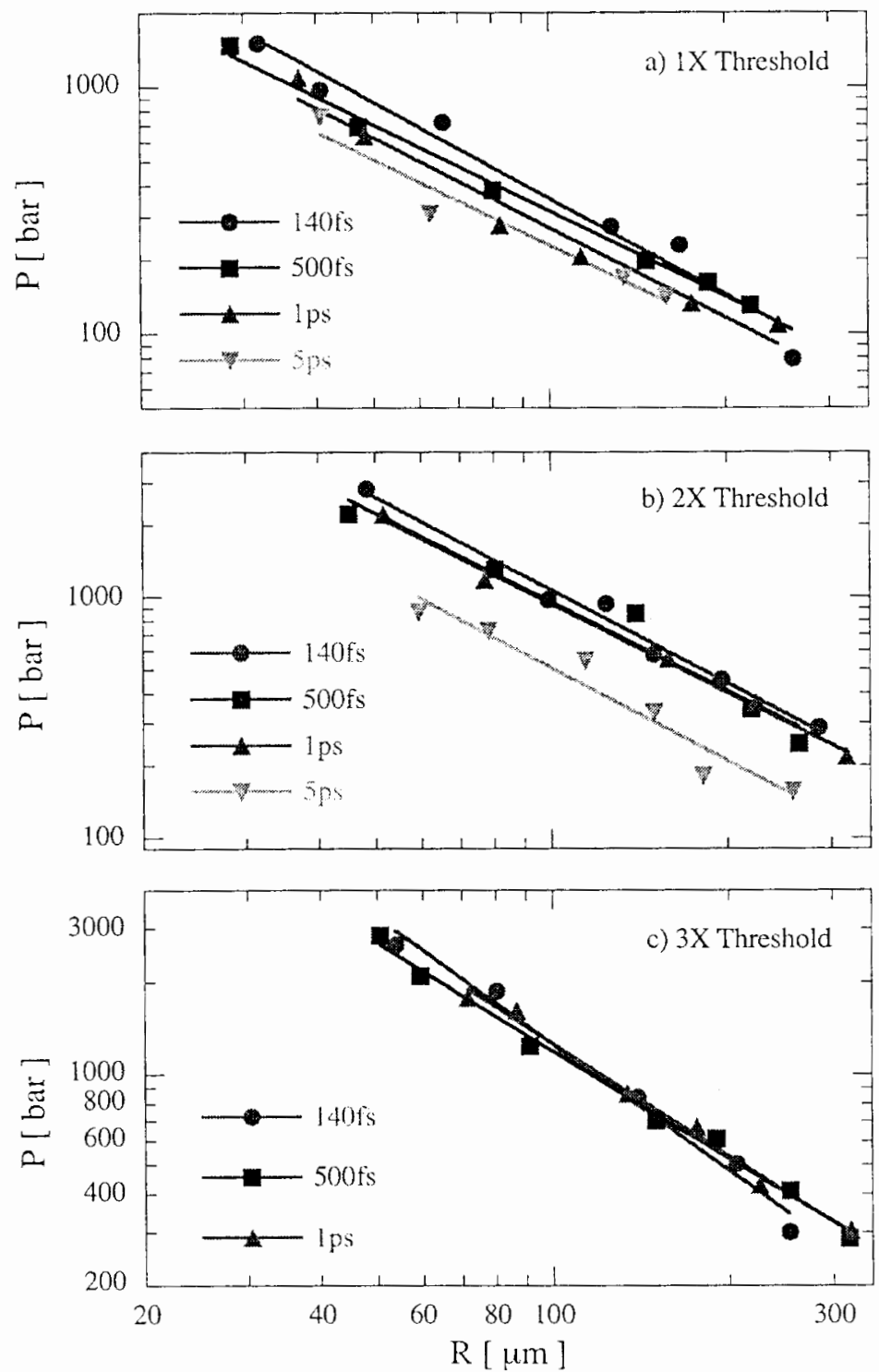

Figure 4.16: Estimated peak pressure as a function of wave radius for $F / F_{t h}=1,2,3$. 
weakly dependent on the pulse width. The pressure decays from approximately 1000bars at $\mathrm{r} \approx 50 \mathrm{um}$ to about $100 \mathrm{bar}$ at $300 \mu \mathrm{m}$ for $F=F_{t h}$, from $2500 \mathrm{bars}$ to $250 \mathrm{bars}$ for $F=2 F_{\text {th }}$, and from 3000 bars to 300 bars for $F=3 F_{t h}$. The decay curves were fit to a power law function $r^{-a}$ and the $\alpha^{\prime}$ s were found to be in the range of 1.15-1.38. Higher pressure pulses had faster decrease in pressure.

We see that pressure decays a bit faster than inverse radius $1 / r$ - the pressure evolution of sound waves in spherical geometry [Zel'dovich67]. There are two factors that may contribute to this behavior. First, even if the shock heating is small, after propagating a considerable distance, the wave does lose some energy. Second, since the symmetry is incomplete (the wave is hemispherical), the pressure must vanish at the water surface due to the boundary condition, and rarefaction waves launched at this boundary reduce the pressure.

The evolution of cylindrical waves is complicated by the fact that the laser beam energy deposition is a function of depth, thus different pressures are generated at a different depth. Since this is a weak shock regime, the propagation speed is constant and the cylindrical shape is preserved. However, there is a constant flow of energy across the shock front that complicates the results. Our measurements at a depth of $200 \mu \mathrm{m}$ indicate that cylindrical waves are noticeably weaker, evolving from approximately 400 bars at $40 \mu \mathrm{m}$ to 100 bars at $200 \mu \mathrm{m}$. The decay is only a little bit slower than $1 / r$, whereas 
according to energy conservation considerations it should be $1 / \sqrt{r}$. It demonstrates that indeed there is energy flow along the symmetry axis of the wave.

Once the pressure and wave profiles are known, we can calculate the energy carried by the wave and estimate the conversion efficiency. For spherical symmetry, the energy can be calculated from the formula [Cole48]

$$
E=2 \pi R^{2} \int \frac{p(t)^{2}}{c_{0} \rho_{0}} d t
$$

where $p(t)$ is the pulse pressure, $c_{0}$ is the sound speed and $\rho_{0}$ is the normal density of water. Since we know the propagation velocity is equal to the sound speed, and the spatial profile of pressure is assumed to have a triangular form, a simple result is obtained

$$
E=\frac{2 \pi \mathrm{R}^{2} p_{\text {peak }}{ }^{2}(R-a)}{3 c_{o}^{2} \rho_{o}} .
$$

For a spherical wave with a triangular profile, the internal shell radius $a=x_{m}^{2} / R$. If we simulate a rectangular profile, $a=R-x_{m}$.

Figure 4.17 presents results of calculations of wave energy for a $140 \mathrm{fs}$ pulse and $200 \mu \mathrm{m}$ shock radius as a function of laser fluence. Measurements show that the fraction of beam energy deposited as mechanical energy (shock wave) is on the order of one percent. A similar calculation was done for the case of the simulated shock shown in Figure 4.13 . The energy carried by the wave is $0.046 \mathrm{~J} / \mathrm{cm}^{2}$. Since the modeling was done for energy deposition of a laser pulse with fluence $4.47 \mathrm{~J} / \mathrm{cm}^{2}$ (Figure 4.8 ), we calculate a 
similar conversion rate of one percent. In this case, about a third of the pulse energy was absorbed in the surface layer, thus conversion of absorbed energy is approximately $3 \%$. This is much lower than the conversion efficiency of $90 \%$ found in volumetric laser induced breakdown of nanosecond pulses [Vogel99]. It also appears to be lower than in the case of volumetric absorption of femtosecond pulses, which is on the order of $10 \%$ [Vogel99,Noack98b].

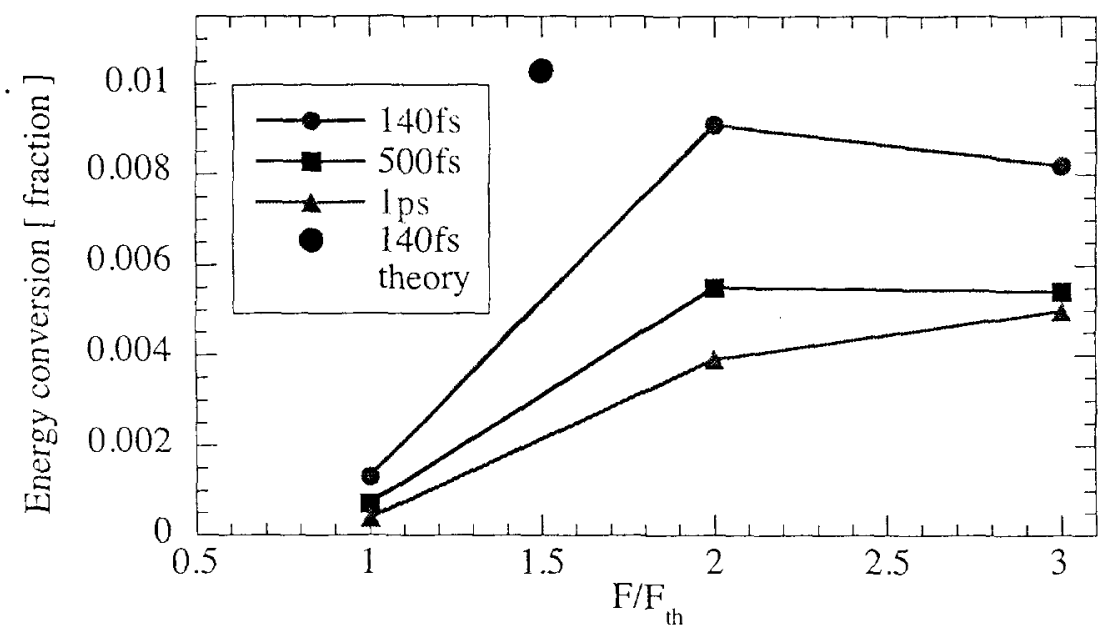

Figure 4.17: Energy conversion efficiency for $140 \mathrm{fs}$ pulse calculated from experimental results and obtained from simulations. Both agree qualitatively and show conversion efficiency less than $1 \%$.

\subsection{Conclusion}

Laser ablation of a water surface generates plasma that allows transmission in the bulk of only the leading edge of the laser pulse. Since the ablation fluence threshold is an increasing function of pulse width, this energy increases for longer pulses and may be 
large enough to cause deleterious effects. Laser ablation can affect the bulk of the material thermally and mechanically. However, for ultrashort pulses these effects are low, because breakdown occurs at relatively low laser energy and absorption is very localized. We also found experimentally and theoretically that the conversion efficiency of USLP laser energy into shock waves is on the order of one percent. A significant factor in decreasing the generated pressure is breakdown at the water surface. The surface allows a significant part of the laser deposited energy to escape with ejecta. Surface ablation creates pressure waves that fall in the so-called weak shock regime. Their velocity is practically equal to the sound speed, therefore interferometry must be used to determine wave strength. 


\section{Chapter 5 Conclusion}

The goal of this work has been to improve our understanding of the interaction of powerful ultrashort pulse laser radiation with simple metals (aluminum, copper) and wide-bandgap dielectrics (fused silica, water) and to develop physical models and tools needed for quantitative modeling of ablation and its effects. The investigations were concentrated on light pulses that are a few picoseconds or shorter in duration and produced by a compact, powerful (up to a terawatt) CPA laser system. In this ultrashort interaction regime, the hot, expanding plasma created by the laser does not have enough time to evolve into a thick plume and shield the material from the laser energy. Since the absorption occurs in very dense plasma present from the beginning in metals or created by the avalanche and nonlinear multiphoton ionization in dielectrics, the thickness of the surface energy deposition layer is small. Therefore it takes a short time for the hot ablation products to expand and separate from the bulk of the material. This effect significantly limits the amount of the energy diffusion and allows the bulk of the material to remain cold. As a result, high processing precision and low collateral damage can be achieved. Such features make ultrashort pulses attractive for many uses. Thus, besides general scientific interest, the need for understanding and optimization of such applications as material removal or laser film deposition motivated my studies. Since the 
physics of the process is complex and nonlinear, most of the results were obtained using numerical modeling.

Examination of the interaction with metals was the first part. As a result of the light absorption the metal temperature may vary many orders of magnitude and the material state changes from a cold solid to hot plasma. Thus it is necessary to have an approach that allows to accommodate such drastic changes. I have presented the needed physical models that are simple, yet catching the essentials of the process, together with their implementation on the basis of a one-dimensional plasma hydrodynamics code. The main focus of the discussion was the unequal electron-ion temperature evolution and laser absorption as a function of lightwave and material properties. It has been shown that the electron temperature can significantly exceed the temperature of the lattice/ions and, in consequence, accelerate many processes - there is a noticeable electron thermal diffusion during the USLP absorption; changes in the absorptivity occur faster due to the effect of electron-electron collisions. At higher temperatures, the electrons create a significant pressure and initiate expansion before the ions are heated. The suggested model for optical properties (dielectric permittivity) demonstrates the significance of interband transitions (electron transitions from one energy band to another) and, when coupled with a wavesolver, allows reproduction of available experimental results for USLP absorption. The developed code gives a self-consistent description of the interaction in a wide range of parameters and I have illustrated effects of laser fluence 
(energy per area), wavelength, pulselength and prepulse, polarization and angle of incidence on laser absorption and deposition.

For a majority of applications, the interest is in the long-term material evolution (time scales much larger than the pulse duration). Using the hydrocode and analytical methods, three different aspects of the ablation have been examined - evolution of the laser-induced pressure pulses, material removal and three-dimensional expansion of the plume. The width of the pressure pulse is determined by the thickness of the deposition zone (not the USLP length). Quick shock formation leads to energy dissipation and, as a result, shock waves are an energy transport mechanism competing with thermal transport. Action of the rarefaction wave and dissipation cause shock pulse decay and spreading as a power law function of the traveled distance. Using the hydrocode, I have demonstrated that the decay exponent changes from -0.5 for weak shocks to -1 for the pulses in the many Mbar range, when their evolution becomes self-similar. To calculate the amount of the material removed, a criterion based on the use of the critical liquid-vapor point was applied to a range of calculations. Removal efficiency (removal per unit of laser energy) has been shown to be the highest for subpicosecond pulses with energy at or near the ablation threshold energy. The ejecta carries away approximately eighty percent of the deposited laser energy. Three-dimensional evolution of the plume was modeled by matching the results of the hydrodynamic calculations with gas ellipsoid model. The 
expansion is very directional, with most of the material being ejected within an angle of $5^{\circ}-10^{\circ}$

Studies of wide-bandgap dielectrics were motivated by the available experimental interferometric study of USLP induced pressure waves in water, which serves as a model of biological tissue. Theoretical modeling based on the nonlinear ionization equation has helped to explain the observed picture by showing that in the USLP surface ablation of wide-bandgap dielectrics laser created plasma acts as a filter that transmits into the bulk only a leading, fixed energy part of the pulse. Since the ablation threshold energy increases with the pulselength, the transmitted energy also increases. Thus it may create potentially unwanted effects, which have been observed in the experiment. Experimental and theoretical study of the three-dimensional pressure wave evolution has demonstrated that near threshold ablation creates pressure waves in the weak shock regime. Conversion efficiency from laser to pressure energy has been shown to be approximately one percent. Systematic study of different aspects of the interaction of powerful ultrashort laser pulses with materials was the purpose of my work. This is an immensely rich and diverse physical phenomenon. Rapid development of technology is constantly presenting new opportunities. The effort to understand and explain is certainly far from over. I would like to suggest possible future directions in advancing the science and applications.

One of them is study of the influence of surface structure on the laser energy absorption. It can be split into two parts. First is understanding effects of surface 
roughness and microstructures. The experiments used to test the hydrocode were performed with vacuum deposited films, which represent an ideal case. In the real world the material surface is certainly not flat. This is especially important for metals, since they have a very thin skin depth. Figure 5.1 shows measurements of cold absorption for aluminum for vacuum deposited films and polished surface.

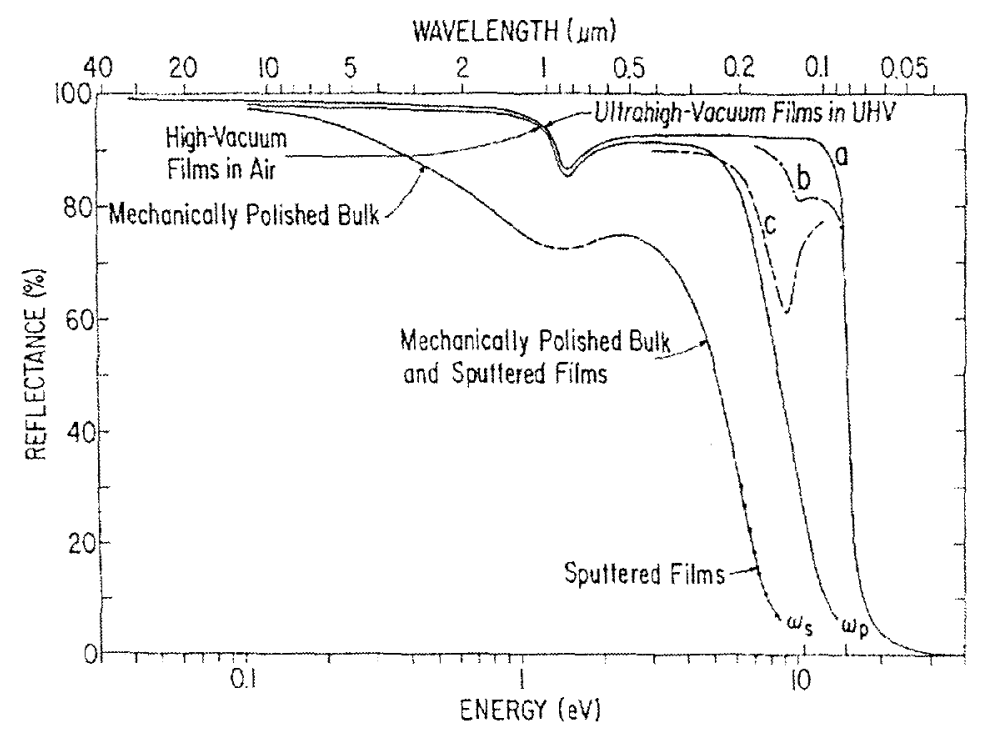

Figure 5.1: Reflectivity of aluminum as a function of wavelength for different material samples. The picture is from Handbook of optical constants of solids, edited by Edward D. Palik [Palik98].

We can see that even a conventionally polished surface is not smooth enough and the absorption is significantly enhanced. One of the explanations for the absorption increase is the excitation of surface plasma waves. These waves are effectively generated if the surface is not flat; they act as an additional laser energy coupling mechanism, besides direct Joule heating and interband transitions. Experiments with USLP ablation of 
premanufactured surface structures [Murnane93, Rajeev02] also show that the absorptivity can be significantly enhanced. The second direction in this study should be understanding of the ultrashort pulse propagation in a channel either metallic or dielectric. This is a very important problem in laser drilling of high aspect ratio holes. The hole acts as a waveguide and one needs to know what modes it supports, how the wall absorbs the light energy and what happens at the bottom of a "blind" hole.

Our examinations of the material removal also did not include three-dimensional effects: This would be material removal through melt splashing or spallation. The melt layer is relatively thin, however, as Figure 3.18 illustrates, it is several times larger than the thickness of the ablated layer (the material removed as a gas). Possible hydrodynamic instabilities at the surface of the melt may initiate ejection of droplets of the material or create surface structures that may affect absorption of the next pulse. Another threedimensional effect that deserves attention is nonequilibrium phase dynamics of the expanding hot liquid. The separation in gas and liquid and formation of vapor bubbles needs to be studied within an approach that properly describes kinetics of the process. Simulations with molecular dynamics codes would be a promising start [Perez02]. Understanding plume composition and size distribution of the particles is very important in laser film deposition, where homogeneity of the film is very important.

One of the major trends in the development of technology is the generation of shorter and shorter pulses. Pulses as short as $10 \mathrm{fs}$ can already be produced and there is a 
discussion of possible generation of single cycle radiation. Since dielectrics do not have free electrons and the plasma has to be created by the USLP, a study of the ionization of dielectrics by the extremely short pulses is needed. Experiments show [Lenzner99] that there is a visible difference in ablation with $5 \mathrm{fs}, 20 \mathrm{fs}$ and $220 \mathrm{fs}$ pulses.

These are just a few possible directions for future studies. Science, technology and applications of laser radiation and ultrashort pulses in particular continue to be at the forefront of research. Undoubtedly there will be many more important and exciting problems to solve. 


\section{References}

Abrikosov72: A.A. Abrikosov, Introduction to the theory of normal metals (Academic Press, 1972).

Andrews85: D.L. Andrews, "A simple statistical treatment of multiphoton absorption," Am. J. Phys. 53(10), 1001(1985).

Anisimov74: S.I. Anisimov, B.L. Kapelovich, and T.L. Perel'man, "Electron emission from metal surfaces exposed to ultrashort laser pulses," Sov. Phys. JETP 39(2), 376 (1974).

Anisimov93: S.I. Anisimov, D. Bäuerle, B.S. Luk'yanchuk, "Gas dynamics and film profiles in pulses-laser deposition of materials," Phys. Rev. B, 48(16) 12076(1993).

Anisimov95: S.I. Anisimov, B.S. Luk'yanchuk, A. Luches, "An analytical model for three-dimensional laser plume expansion into vacuum in hydrodynamic regime," Appl. Surf. Sci. 96-98, 24(1996).

Anisimov97: S.I. Anisimov and B. Rethfeld, "On the theory of ultrashort laser pulse interaction with metal," Izv. Akad. Nauk Fiz+ 61(8),1642(1997).

Arnold84: G.S. Arnold, "Absorptivity of several metals at $10.6 \mu \mathrm{m}$ : empirical expressions for the temperature dependence computed from Drude theory," Appl. Opt. 23(9), 1434(1984).

Artsimovich93: L. A. Artsimovich, Plasma physics for physicists (Springer Verlag,1993).

Ashcroft71: N.W. Ashcroft and K. Sturm, "Interband absorption and the optical properties of polyvalent metals", Phys. Rev. B 3, 1898 (1971).

Backus97: Sterling Backus, Charles G. Durfee III, Gerard Mourou, Henry C. Kapteyn, and Margaret M. Murnane, "0.2-TW laser system at $1 \mathrm{kHz}$," Optics Lett. 22, 1256 (1997)

Backus98: Sterling Backus, Charle G. Durfee III, Margaret M. Murnane, and Henry C. Kapteyn, "High power ultrafast lasers," Rev. Sci. Instrum. 69, 1207 (1998).

Bagnoud00: V. Bagnoud, F. Salin, "Amplifying laser pulses to the terawatt level at a 1kilohertz repetition rate," Appl. Phys. B 70, S165 (2000)

Banks99: P.S. Banks, L. Dinh, B.C. Stuart, M.D. Feit, A.M. Komashko, A.M. Rubenchik, M.D. Perry, W. McLean, "Short-pulse laser deposition of diamond-like carbon thin films," Appl. Phys. A 69S, 347 (1999). 
Basov63: N.G. Basov and O.N. Krokhin, in Proceedings of the conference on quantum electronics, Paris 1963.

Bauerle00: Dieter Bäuerle, Laser processing and chemistry (Springer, Berlin, 2000).

Blaha00: P. Blaha, K. Schwarz, G. Madsen, D. Kvasnicka and J. Luitzelectronic, The program package WIEN2k for structure calculations of solids using density functional theory, http://www.wien2k.at/index.html

Bloembergen74: Nicolaas Bloembergen, "Laser-induced electric breakdown in solids," IEEE J. Quantum Electron. QE-10(3), 375(1974).

Born89: M. Born and E. Wolf, Principles of optics: electromagnetic theory of propagation, interference, and diffraction of light (Pergamon Press, Oxford, 1989).

Bruckner89: M. Bruckner, J.H. Schafer, and J. Uhlenbusch, "Ellipsometric measurement of the optical constants of aluminum and copper at $\lambda=10.6 \mu \mathrm{m}, " \mathrm{~J}$. Appl. Phys. 66(3), 1326(1989).

Brysk74: H. Brysk, "Electron-ion equilibration in a partially degenerate plasma," Plasma Phys., 16, 927(1974).

Campbell90: J.H. Campbell, F. Rainer, M. Kozlowski, C.R. Wolfe, I. Thomas, and F. Milanovich, "Damage resistant optics for a mega-joule solid-state laser," Proc. SPIE 1441, 444(1990).

Carslaw86: H.S. Carslaw and J.C. Jaeger, Conduction of heat in solids (Oxford University Press, 1986).

Chichkov96: B.V. Chichkov, C. Momma, S. Nolte, F. von Alvensleben, A. Tunnermann, "Femtosecond, picosecond and nanosecond laser ablation of solids," Appl. Phys. A 63(2), 109 (1996).

Cole48: R.H. Cole, Underwater Explosions (Princeton University Press, Princeton, NJ, 1948).

Courant77: R. Courant and K.O. Friedrichs, Supersonic flow and shock waves (Springer, New York, 1977).

Dane02: "Bright future for tactical laser weapons", LLNL Science and Technology

Review, p.19, April 2002. http://www.llnl.gov/str/April02/Dane.html

Davis93: ASM specialty handbook: aluminum and aluminum allows, edited by J.R. Davis (ASM International, 1993). 
DeSalvo96: Richard DeSalvo, Ali A. Said, David J. Hagan, Eric W. Van Stryland, and Mansoor Sheik-Bahae, "Infrared to ultraviolet measurements of two-photon absorption and $n_{2}$ in wide bandgap solids," IEEE J. Quantum Electron. QE-32(8), 1324 (1996).

Ditmire96: T.R. Ditmire, "Soft X-ray generation in gases with an ultrashort pulse laser," Ph.D. Dissertation, University of California, Davis 1996.

Docchio88: F. Docchio, "Lifetime of plasmas induced in liquids and ocular media by single Nd:YAG laser pulses of different duration," Europhys. Lett., 6(5), 407(1988).

Doukas96: A.G. Doukas and T.J. Flotte, "Physical characteristics and biological effects of laser-induced stress waves," Ultrasound Med. Biol., 22(2), 151(1996).

Drogoff01: B. Le Drogoff, J. Margot, M. Chaker, M. Sabsabi, O. Barthelemy, T.W. Johnston, S. Laville, F. Vidal, Y. von Kaenel, "Temporal characterization of femtosecond laser pulse induced plasma for spectrochemical analysis of aluminum alloys," Spectrochimica Acta Part B 56, 987 (2001).

Du94: D. Du, X. Liu, G. Korn, J. Squier, and G. Mourou, "Laser-induced breakdown by impact ionization in $\mathrm{SiO}_{2}$ with pulse widths from 7 ns to $150 \mathrm{fs}$," Appl. Phys. Lett, 64(23), 3071(1994).

Ehrenreich66: H. Ehrenreich, in The optical properties of solids, edited by J. Tauc (Academic Press, New York, 1966).

Eidmann 00: K. Eidmann, J. Meyer-ter-Vehn, T. Schlegel, and S. Huller, "Hydrodynamic simulation of subpicosecond laser interaction with solid-density matter", Phys. Rev. E 62, 1202 (2000).

Elsayed-Ali87: H.E. Elsayed-Ali, T.B. Norris, M.A. Pessot, and G.A. Mourou, "Timeresolved observation of electron-phonon relaxation in copper", Phys. Rev. Lett., 58(12), 1212(1987).

Feit01: M.D. Feit, A.M. Rubenchik, M. Runkel, "Analysis of bulk DKDP damage distribution, obscuration and pulse length dependence", Proc. SPIE Vol. 4347, 383(2001).

Feller62: W. Feller, An introduction to probability theory and its applications (John Wiley \& Sons, Inc., New York, 1962).

Feynman49: R.P. Feynman, N. Metropolis, and E. Teller, "Equations of state of elements base on generalized Fermi-Thomas theory," Phys. Rev., 75(10), 1561(1949).

Fisher01: D. Fisher, M. Fraenkel, Z. Henis, E. Moshe, and S. Eliezer, "Interband and intraband (Drude) contributions to femtosecond laser absorption in aluminum", Phys. Rev. E 65, 016409 (2001). 
Gibbon96: P. Gibbon and E. Förster, "Short-pulse laser-plasma interactions," Plasma Phys. Control. Fusion, 38, 769(1996).

Ginzburg55: V.L. Ginzburg and V.P. Shabanskii, "Kinetic temperature of electrons and anomalous electron emission," Dok1. Akad. Nauk SSSR 100, 445 (1955), in Russian.

Ginzburg70: V.L. Ginzburg, The propagation of electromagnetic waves in plasmas (Pergamon Press, 1970).

Glezer97: E.N. Glezer, C.B. Schaffer, N. Nishimura, E. Mazur, "Minimally disruptive laser-induced breakdown in water," Opt. Lett., 22(23), 1817(1997).

Grady88: D.E. Grady, "The spall strength of condensed matter," J. Mech. Phys. Solids, 36(3), 353(1988).

Gray63: American institute of physics handbook, edited by Dwight E. Gray (McGrawHill, 1963).

Grover71: R. Grover, "Liquid metal equation of state based on scaling", J. Chem. Phys. 55, 3435 (1971).

Hammer96: D.X. Hammer, R.J. Thomas, G.D. Noojin, B.A. Rockwell, P.K. Kennedy, W.P. Roach, "Experimental investigation of ultrashort pulse laser-induced breakdown thresholds in aqueous media," IEEE J. Quantum Electron., QE-32(4), 670(1996).

Henis93: Z. Henis and S. Eliezer, "Melting phenomenon in laser-induced shock waves," Phys. Rev. E, 48(3), 2094(1993).

Hu96: X.-H. Hu, T. Juhasz, "Study of corneal ablation with picosecond laser pulses at $211 \mathrm{~nm}$ and $263 \mathrm{~nm}$," Lasers Surg. Med., 18(4), 373(1996).

Huttner94: B. Huttner, "Optical properties of polyvalent metals in the solid and liquid state: aluminum", J. Phys.: Condens. Matter, 6, 2459(1994).

Huttner95: B. Huttner, "Optical properties of polyvalent metals in the solid and liquid state: lead", J. Phys.: Condens. Matter, 7, 907(1995).

Iida88: T. Mida, Physical properties of liquid metals(Oxford University Press, 1988).

Iserles96: A. Iserles, A first course in the numerical analysis of differential equations (Cambridge University Press, 1996).

Jackson98: J.D. Jackson, Classical electrodynamics (John Wiley \& Sons, 1998).

Jiang95: Z. Jiang, J.C. Kieffer, J.P. Matte, M. Chaker, O. Peyruse, D. Gilles, G. Korn, A. Maksimchuk, S. Coe, and G. Mourou, "X-ray spectroscopy of hot solid density plasmas produced by subpicosecond high contrast laser pulses at $10^{18}-10^{19} \mathrm{~W} / \mathrm{cm}^{2}$," Phys. Plasmas, 2(5), 1702 (1995). 
Juhasz96: T. Juhasz, X.-H. Hu, L. Turi, Z. Bor, "Dynamics of shock-waves and cavitation bubbles generated by picosecond laser-pulse in corneal tissue and water," Lasers Surg. Med., 15(1), 91(1994).

Kaganov57: M.I. Kaganov, I.M. Lifshitz, and L.V. Tanatarov, "Relaxation between electrons and crystalline lattice," Sov. Phys. JETP 4, 173 (1957).

Keldysh65: L.V. Keldysh, "Ionization in the field of a strong electromagnetic wave,' Sov. Phys. JETP 20(5), 1307 (1965).

Kennedy95: P.K. Kennedy, "A first-order model for computation of laser-induced breakdown threshold in ocular and aqueous media: part I - theory," IEEE J. Quantum Electron., QE-31(12), 2241(1995).

Key91: M.H. Key, in Handbook of plasma physics, Vol. 3: Physics of laser plasma, edited by A. Rubenchik, S. Witkowski (Elsevier Science Publishers B.V., Amsterdam, 1991).

Kieffer93: J.C. Kieffer, M. Chaker, J.P. Matter, H. Pepin, C.Y. Cote, Y. Beaudoin, T.W. Johnston, C.Y. Chien, S. Coe, G. Mourou, O. Peyrusse, "Ultrafast X-ray sources," Phys. Fluids B 5(7), 2676 (1993).

Kim01: B.-M. Kim, M.D. Feit, A.M. Rubenchik, E.J. Joslin, P.M. Celliers, J. Eichler, L.B. Da Silva, "Influence of pulse duration on ultrashort laser pulse ablation of biological tissues," J. Biomed. Optics, 6(3), 332(2001).

Kim02: B.-M. Kim, A.M. Komashko, A.M. Rubenchik, M.D. Feit, J. Eichler, S. Reidt, P.M. Celliers and L.B. Da Silva, "Interferometric analysis of ultrashort pulse laserinduced pressure waves in water," Submitted to Journal of Applied Physics.

Kinslow70: High-velocity impact phenomena, edited by Ray Kinslow (Academic Press, 1970).

Kittel96: Charles Kittel, Introduction to solid state physics (Wiley, Toronto, 1996).

Komashko00: A.M. Komashko, M.D. Feit, A.M. Rubenchik, "Modeling of long-term behavior of ablation plumes produced with ultrashort laser pulses," Proc. SPIE 3935, 97 (2000).

Komashko99: A.M. Komashko, M.D. Feit, A.M. Rubenchik, M.D. Perry, P.S. Banks, "Simulation of material removal efficiency with ultrashort laser pulses," Appl. Phys. A V69S, 95(1999).

Krishnan93a: S. Krishnan and P.C. Nordine, "Optical properties of liquid aluminum in the energy range 1.2-3.5eV," Phys. Rev. B, 47(18), 11780(1993). 
Krishnan93b: S. Krishnan and P.C. Nordine, "Analysis of the optical properties of liquid aluminum", Phys. Rev. B, 48(6), 4130(1993).

Landau59: L.D. Landau and E.M. Lifshitz, Fluid mechanics (Pergamon Press, 1959).

Landau69: L.D. Landau and E.M. Lifshitz, Statistical physics (Addison-Wesley, 1976).

Landau76: L.D. Landau and E.M. Lifshitz, Mechanics (Pergamon Press, 1976).

Larsen01: J.T. Larsen, Book II: the physics in HYADES, Report CAS062, (Cascade Applied Sciences, Inc., Boulder, CO, 2001).

Larsen91: J.T. Larsen, in Radiative properties of hot dense matter (World Scientific, Singapore, 1991).

Larsen94: J.T. Larsen and S.M. Lane, "HYADES - a plasma hydrodynamics code for dense plasma studies," J. Quant. Spectrosc. Radiat. Transfer, 51, 179(1994).

Lee84: Y.T. Lee and R.M. More, "An electron conductivity model for dense plasmas," Phys. Fluids, 27(5), 1273(1984).

Lenzner99: M. Lenzner, J. Kruger, W. Kautek, F. Krausz, "Precision laser ablation of dielectrics in the 10fs regime," Appl. Phys. A 68, 369(1999).

Lifshitz81: E.M. Lifshitz and L.P. Pitaevskii, Physical kinetics (Pergamon Press, 1981).

Lind195: John Lind1, "Development of the indirect-drive approach to inertial confinement fusion and the target physics for ignition and gain," Phys. Plasmas 2(11), 3933 (1995).

Liu97: X. Liu, D. Du, G. Mourou, "Laser ablation and micromachining with ultrashort laser pulses," IEEE J. Quantum Electron. 23(10), 1706 (1997).

Loesel96: F.H. Loesel, M.H. Niemz, J.F. Bille, T. Juhasz, "Laser-induced optical breakdown on hard and soft tissues and its dependence on the pulse duration: Experiment and model," IEEE J. Quantum Electron. 32(10), 1717 (1996).

Loesel98: F.H. Loesel, J.P. Fischer, M.H. Gotz, C. Horvath, T. Juhasz, F. Noack, N. Suhm, J.F. Bille, "Non-thermal ablation of neural tissue with femtosecond laser pulses," Appl. Phys. B 66(1), 121 (1998).

Louisell73: W.H. Louisell, Quantum statistical properties of radiation (John Wiley \& Sons, Inc., New York, 1973).

Maiman60: T.H. Maiman, "Stimulated optical radiation in ruby", Nature 187, 493 (1960). 
Maine88: P. Maine, D. Strickland, P. Bado, M. Pessot, and G. Mourou, "Generation of ultrahigh peak power pulses by chirped pulse amplification," IEEE J. Quantum Electron. QE-24(2), 398 (1988).

Marburger75: J.H. Marburger, "Self-focusing: Theory," Prog. Quant. Electron. 4, 35(1975).

Margetic00: V. Margetic, A. Pakulev, A. Stockhaus, M. Bolshov, K. Niemax, R. Hergenroder, "A comparison of nanosecond and femtosecond laser-induced plasma spectroscopy of brass samples," Spectrochimica Acta Part B 55, 1771 (2000).

Marsh80: LASL shock Hugoniot data, edited by P.Marsh (University of California Press, 1980).

Martinez87: Oscar Eduardo Martinez, "Design of high-power ultrashort pulse amplifiers by expansion and recompression," IEEE J. Quantum Electron. QE-23(8), 1385 (1987).

Milchberg88: H.M. Milchberg, R.R. Freeman, S.C. Davey, and R.M. More, "Resistivity of a simple metal from room temperature to $10^{6}$ K." Phys. Rev. Lett. 612364 (1988).

Milchberg89: H.M. Milchberg and R.R. Freeman, "Light absorption in ultrashort scale length plasmas," J. Opt. Soc. Am. B, 6(7), 1351(1989).

Miller98: Experimental methods in the physical sciences, Vol. 30: Laser ablation and desorption, edited by J.C. Miller and R.F. Haglund, Jr (Academic Press, San Diego CA, 1998).

More88: R.M. More, K.H. Warren, D.A. Young, and G.B. Zimmerman, "A new quotidian equation of state (QEOS) for hot dense matter", Phys. Fluids 31, 3059 (1988).

More91: R.M. More, in Handbook of plasma physics, Vol. 3: Physics of laser plasma, edited by A. Rubenchik, S. Witkowski (Elsevier Science Publishers B.V., Amsterdam, 1991).

Mott58: N.F. Mott, The theory of the properties of metals and allows (Dover Publications, New York, 1958).

Mourou98: G.A. Mourou, C.P.J. Barty, M.D. Perry, "Ultrahigh-intensity lasers: physics of the extreme on a tabletop," Physics Today 51(1), 22 (1998).

Murnane93: M.M. Murnane, H.C. Kapteyn, S.P. Gordon, J. Bocor, E.N. Glytsis, R.W. Falcone, "Efficient coupling of high-intensity subpicosecond laser pulses into solids," Appl. Phys. Lett. 62(10), 1068(1993).

Murnane94: M.M. Murnane, H.C. Kapteyn, S.P. Gordon, R.W. Falcone, "Ultrashort Xray pulses," Appl. Phys. B 58, 261 (1994). 
Oraevsky96: A.A. Oraevsky, L.B. Da Silva, A.M. Rubenchik, M.D. Feit, M.E. Glinsky, M.D. Perry, B.M. Mammini, W. Small IV, and B.C. Stuart, "Plasma mediated ablation of biological tissues with nanosecond-to-femtosecond laser pulses: relative role of linear and nonlinear absorption," IEEE J. Sel. Top. Quantum Electron. 2(4), 801(1996).

Ovsiannikov82: L.V. Ovsiannikov, Group analysis of differential equations (Academic Press, 1982).

Palik98: Handbook of optical constants of solids, edited by Edward D. Palik (Academic Press, San Diego, CA, 1998).

Pennington00: D.M. Pennington, C.G. Brown, T.E. Cowan, S.P. Hatchett, E. Henry, S. Herman, M. Kartz, M. Key, J. Koch, A.J. MacKinnon, M.D. Perry, T.W. Phillips, M. Roth, T.C. Sangster, M. Singh, R.A. Snavely, M. Stoyer, B.C. Stuart, S.C. Wilks, "Petawatt laser system and experiments," IEEE Journal of Selected Topics in Quantum Electronics 6, 678 (2000).

Perez02: D. Perez and L.J. Lewis, "Ablation of solids under femtosecond laser pulses," Phys. Rev. Lett., 89(25), 255504(2002).

Perry94: Michael D. Perry and Gerard Mourou, "Terawatt to petawatt subpicosecond lasers," Science 264, 917 (1994).

Perry98: M.D. Perry, B.C. Stuart, P.S. Banks, M.D. Feit, J.A. Sefcik, "Ultrashort-pulse laser machining," in LIA handbook of laser material processing, edited by J.F. Ready, et.al. (Laser Institute of America, Orlando FL, 2001).

Perry99: M.D. Perry, D. Pennington, B.C. Stuart, G. Tiethohl, J.A. Britten, C. Brown, S. Herman,B. Golick, M. Kartz, J. Miller, H.T. Powell, M. Vergino, V. Yanovsky, "Petawatt laser pulses," Optics Lett. 24, 160 (1999).

Perry99a: M.D. Perry, B.C. Stuart, P.S. Banks, M.D. Feit, V. Yanovsky, and A.M. Rubenchik, "Ultrashort-pulse laser machining of dielectric materials," J. Appl. Phys. 86(9), 6803 (1999).

Price95: D.F. Price, R.M. More, R.S. Walling, G. Guethlein, R.L. Shepherd, R.E. Stewart, and W.E. White, "Absorption of ultrashort laser pulses by solid targets heated rapidly to temperatures 1-1000ev," Phys. Rev. Lett. 75, 252 (1995).

Prokhorov90: A.M. Prokhorov, V.I. Konov, I. Ursu and I.N. Mihailescu, Laser heating of metals (Adam Hilger, Bristol, 1990).

Rajeev02: P.P. Rajeev, S. Banerjee, A.S. Sandhu, R.C. Issac, L.C. Tribedi, and G.R. Kumar, "Role of surface roughness in hard-x-ray emission from femtosecond-laserproduced copper plasmas," Phys. Rev. A, 65, 052903(2002). 
Stahrenberg01: K. Stahrenberg, Th. Herrmann, K. Wilmers, N. Esser, and W. Richter, "Optical properties of copper and silver in the energy range 2.5-9.0eV," Phys. Rev. B, 64, 115111(2001).

Starrett00: B. Starrett, "How CD-R and CD-RW recording works," Roxio Newsletters, June 12, 2000, http://www.roxio.com/en/support/cdr/howrecworks.html.

Steinberg91: D.J. Steinberg, "Equation of state and strength properties of selected materials", LLNL Report UCRL-MA-106439 (1991).

Strickland85: Donna Strickland and Gerard Mourou, "Compression of amplified chirped optical pulses," Opt. Commun. 33(3), 219 (1985).

Stuart95: B.C. Stuart, M. D. Feit, A. M. Rubenchik, B. W. Shore, and M. D. Perry, "Laser-induced damage in dielectrics with nanosecond to subpicosecond pulses," Phys. Rev. Lett, 74(12), 2248(1995).

Stuart96: B.C. Stuart, M.D. Feit, S. Herman, A.M. Rubenchik, B.W. Shore, and M.D. Perry, "Nanosecond to femtosecond laser-induced breakdown in dielectrics," Phys. Rev. $\mathrm{B}$, 53(4), 1749(1996).

Thormahlen85: I. Thormahlen, J. Straub, and U. Grigull, "Refractive index of water and its dependence on wavelength, temperature and density," J. Phys. Chem. Ref. Data, 14(4), 933(1985).

Tolstoy01: Alexei Tolstoy, The Garin death ray (University Press of the Pacific, 2001; originally published in 1927).

Treacy 69: Edmond B. Treacy, "Optical pulse compression with diffraction gratings," IEEE J. Quantum Electron. QE-5(9), 454 (1969).

Trubnikov65: B.A. Trubnikov, in Reviews of plasma physics vol.1, edited by M. A. Leontovich (Consultants Bureau, New York, 1965).

Vidal01: F. Vidal, T.W. Johnston, S. Laville, O. Barthelemy, M. Chaker, B. Le Drogoff, J. Margot, and M. Sabsabi, "Critical-point phase separation in laser ablation of conductors”, Rhys. Rev. Lett. 86, 2573 (2001).

Vogel94a: A. Vogel, S. Busch, K. Jungnickel, R. Birngruber, "Mechanics of intraocular photodisruption with picosecond and nanosecond laser-pulses," Lasers Surg. Med., 15(1), 32(1994).

Vogel94b: A. Vogel, M.R.C. Capon, M.N. Asiyovogel, R. Birngruber, "Intraocular photodisruption with picosecond and nanosecond laser pulses - tissue effects in cornea, lens, and retina," Invest. Ophthalmol. Visual Sci., 35(7), 3032(1994). 